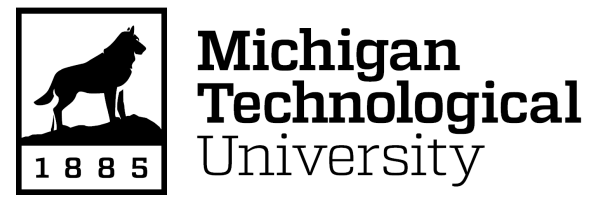

Michigan Technological University Digital Commons @ Michigan Tech

Dissertations, Master's Theses and Master's Reports

2018

\title{
ACUTE AND CHRONIC RESPONSES TO EXERCISE WITH BLOOD FLOW RESTRICTION
}

Matthew A. Kilgas

Michigan Technological University, makilgas@mtu.edu

Copyright 2018 Matthew A. Kilgas

\section{Recommended Citation}

Kilgas, Matthew A., "ACUTE AND CHRONIC RESPONSES TO EXERCISE WITH BLOOD FLOW

RESTRICTION", Open Access Dissertation, Michigan Technological University, 2018.

https://doi.org/10.37099/mtu.dc.etdr/737

Follow this and additional works at: https://digitalcommons.mtu.edu/etdr

Part of the Sports Sciences Commons 


\title{
RESTRICTION
}

By

\author{
Matthew A. Kilgas
}

\begin{abstract}
A DISSERTATION
Submitted in partial fulfillment of the requirements for the degree of DOCTOR OF PHILOSOPHY

In Integrative Physiology
\end{abstract}

MICHIGAN TECHNOLOGICAL UNIVERSITY

2018

(C) 2018 Matthew A. Kilgas 
This dissertation has been approved in partial fulfillment of the requirements for the Degree of DOCTOR OF PHILOSOPHY in Integrative Physiology.

Department of Kinesiology and Integrative Physiology

Dissertation Advisor: Dr. Steven Elmer

Committee Member: Dr. Tejin Yoon

Committee Member: Dr. Min Wang

Committee Member: $\quad$ Dr. John McDaniel

Department Chair: Dr. Megan Frost 


\section{Table of Contents}

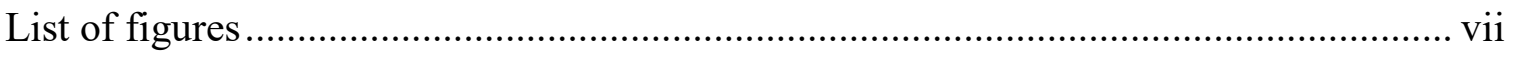

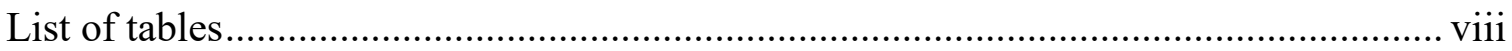

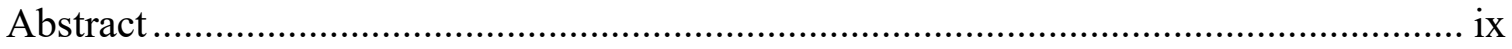

$1 \quad$ Evidenced based guidelines for exercise with BFR ..............................................

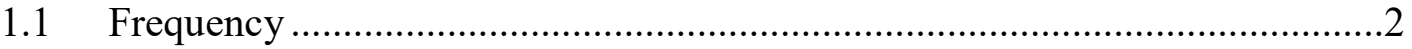

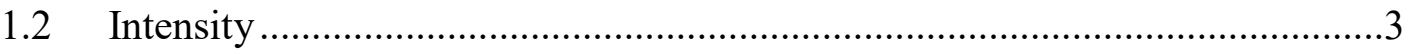

1.3 Time

1.4 Type

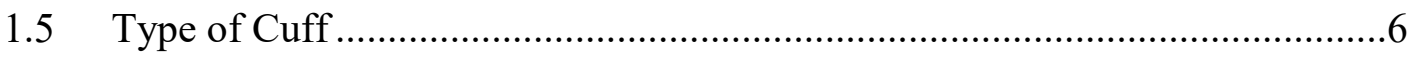

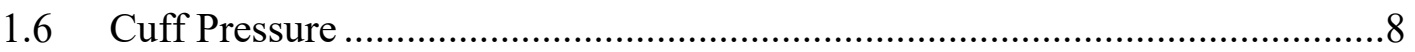

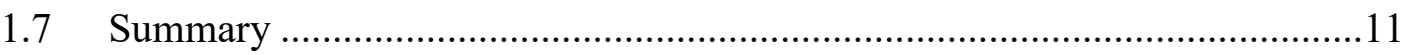

1.8 Gaps and Future Directions .................................................................13

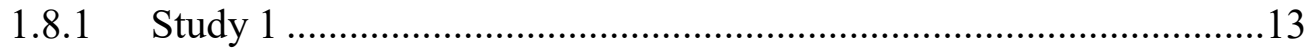

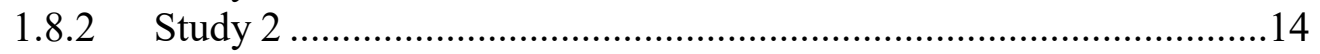

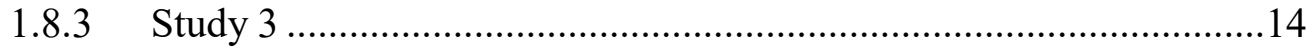

2 Limb Blood Flow and Tissue Perfusion during Exercise with Blood Flow

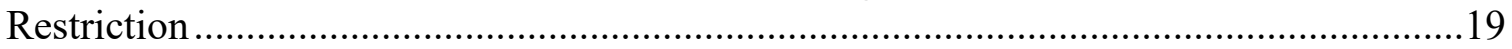

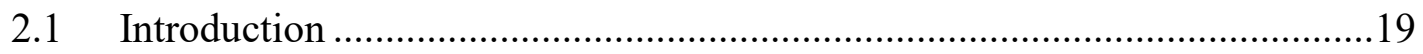

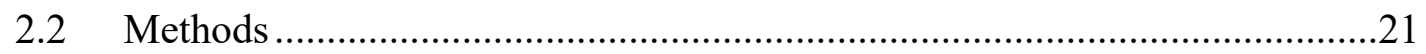

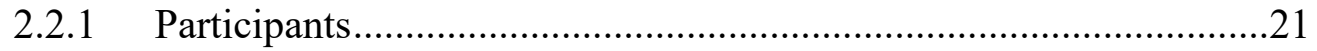

2.2.2 Study Design and Overview ………………................................22

2.2.3 Rhythmic Handgrip Exercise .....................................................23

2.2.4 Blood Flow Calculation .............................................................23

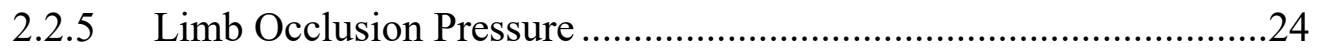

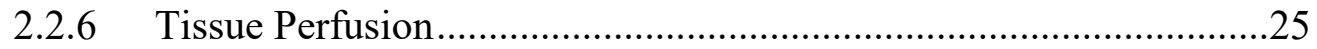

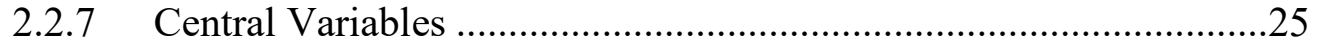

2.2.8 Forearm Muscle Activity ...............................................................26

2.2.9 Statistical Analysis........................................................................26

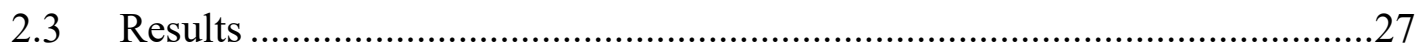

2.3.1 Limb Occlusion and Cuff Pressure ………………............................2

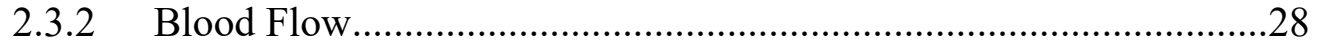

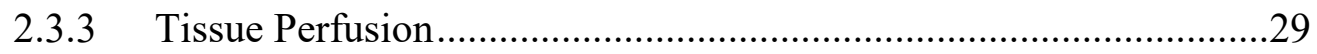

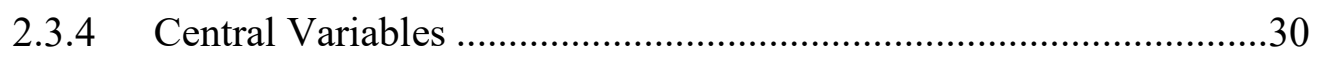




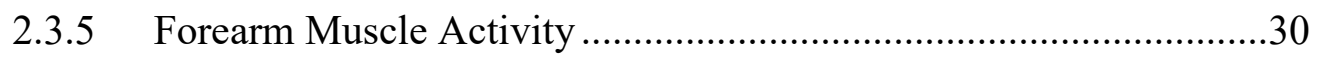

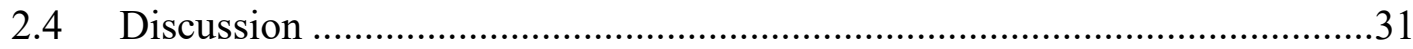

2.4.1 Assessment of Blood Flow during Exercise ........................................31

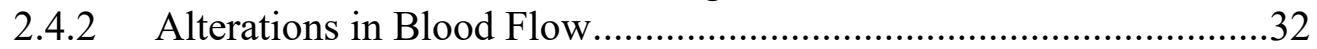

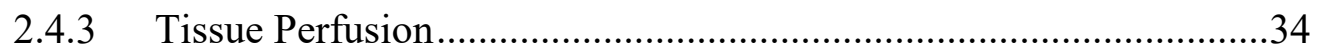

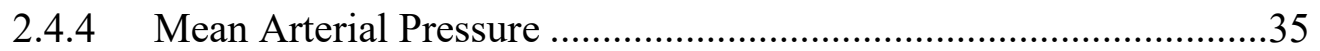

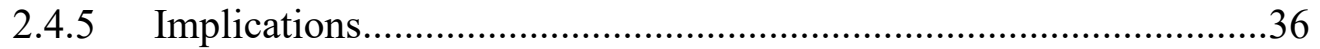

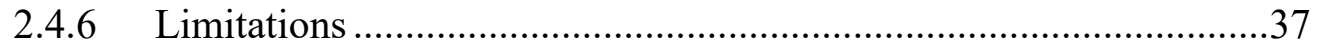

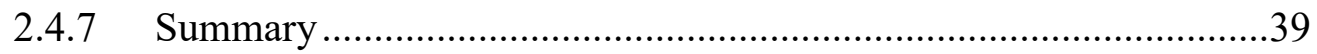

3 Acute Cardiovascular, Metabolic, and Neuromuscular Effects of Cycling with

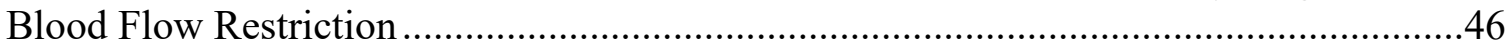

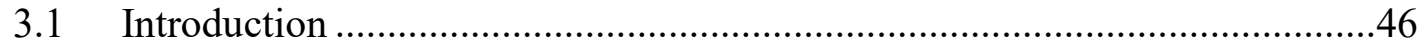

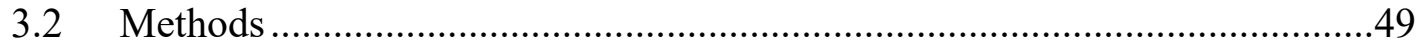

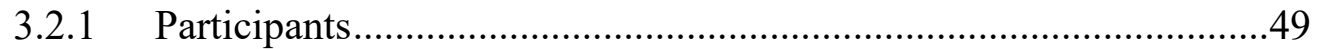

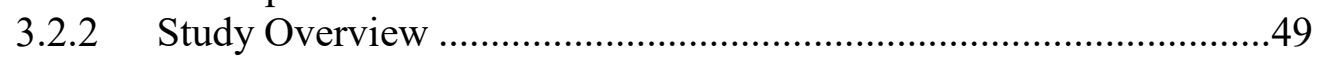

3.2.3 Oxygen consumption and Peak Aerobic Power................................50

3.2.4 Cycling Exercise .........................................................................

3.2.5 Blood Flow..................................................................................

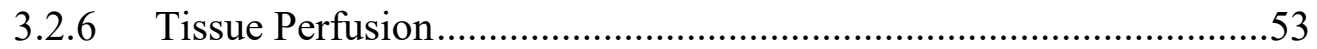

3.2.7 Metabolic, Cardiorespiratory, and Perceptual Responses...................54

3.2.8 Neuromuscular Function..............................................................54

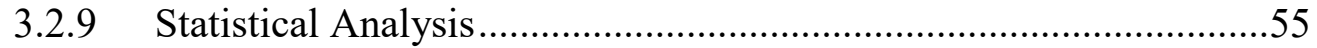

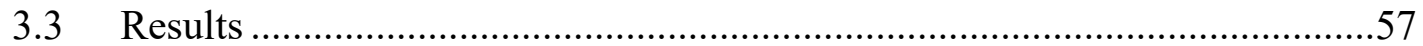

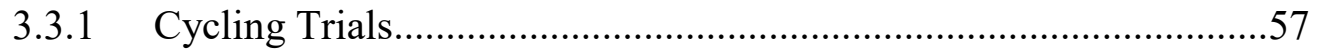

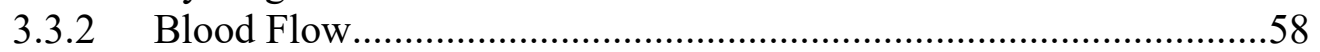

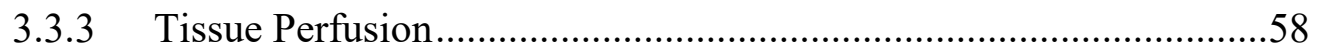

3.3.4 Metabolic, Cardiovascular, and Perceptual Responses......................59

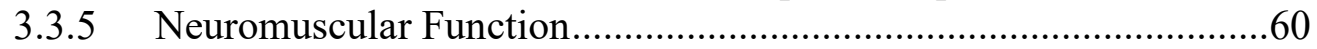

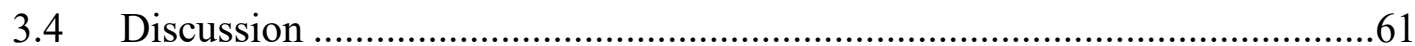

3.4.1 Blood Flow and Tissue Perfusion .....................................................62

3.4.2 Metabolic, Cardiorespiratory, and Perceptual Responses...................63

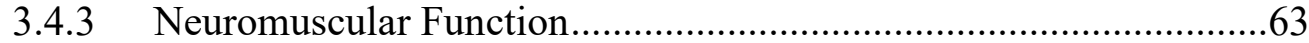

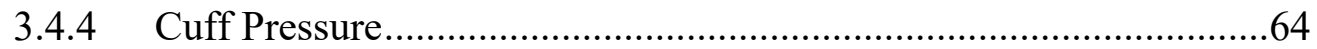

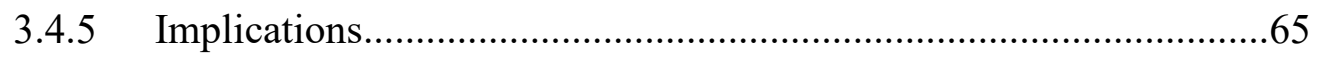

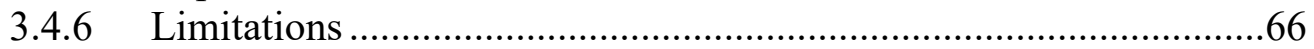

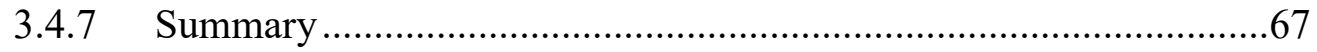

72

4 Adaptations to Aerobic and Resistance Training with Blood Flow Restriction.......74

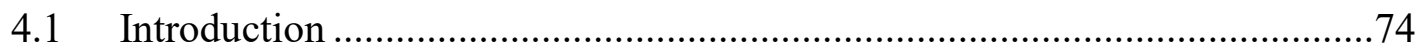




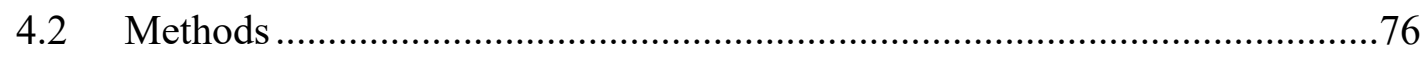

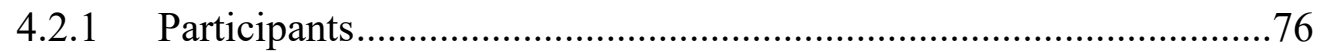

4.2.2 Study Overview ......................................................................

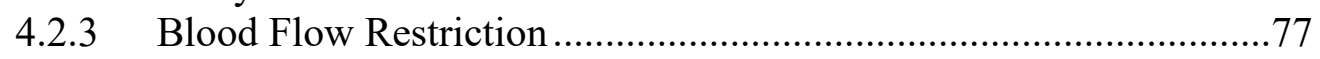

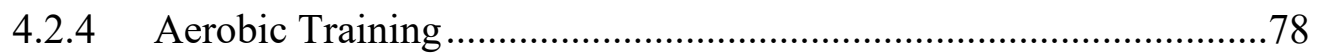

4.2.5 Resistance Training...................................................................78

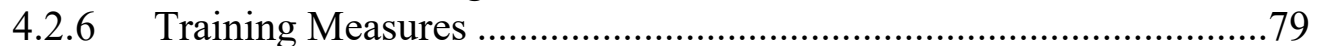

4.2.7 Training Outcome Measures.......................................................79

4.2.7.1 Body Composition ........................................................79

4.2.7.2 Muscle Thickness..............................................................

4.2.7.3 Muscular Strength.......................................................... 81

4.2.7.4 Aerobic Capacity ………………………….................82

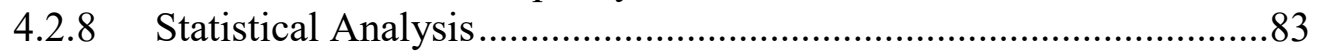

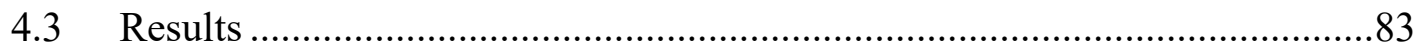

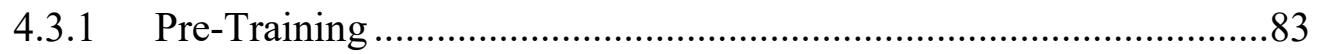

4.3.2 Blood Flow Restriction Training ……………….............................. 84

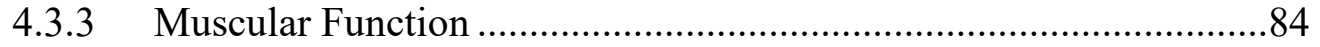

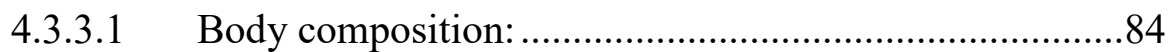

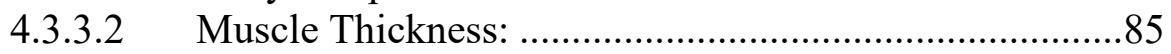

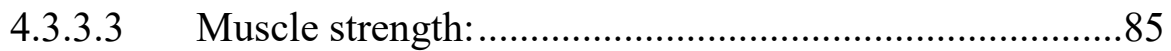

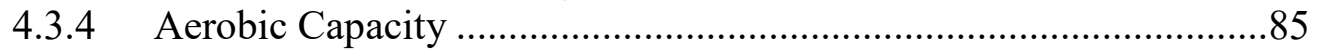

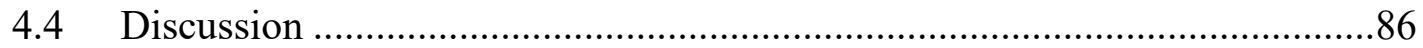

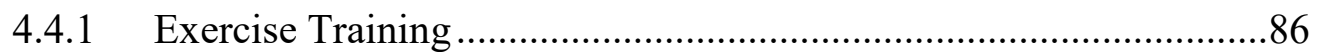

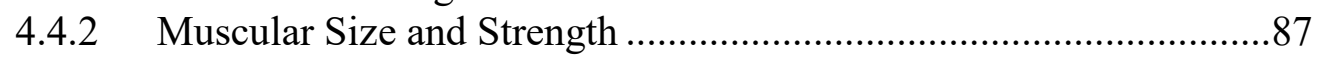

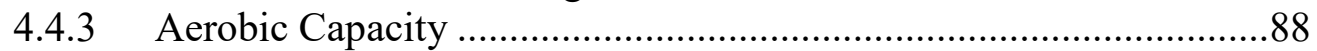

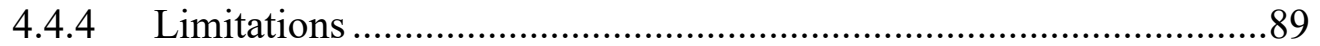

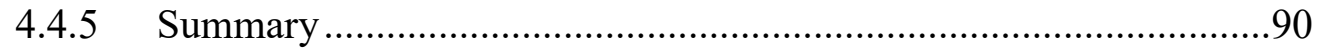

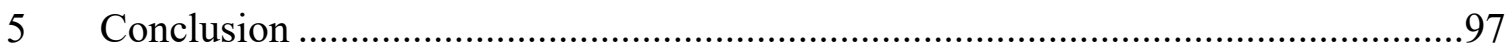

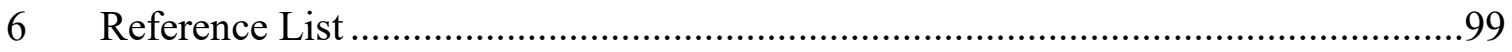

A Approved IRB forms............................................................................111

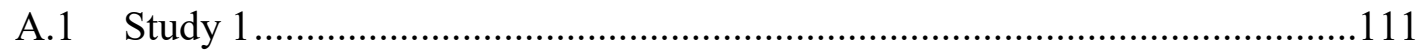

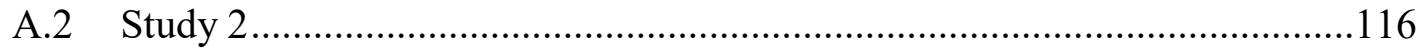

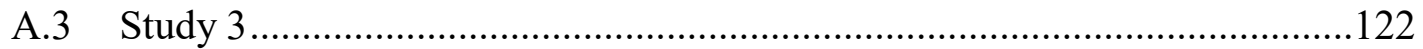

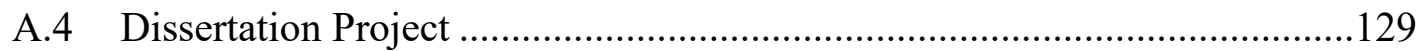

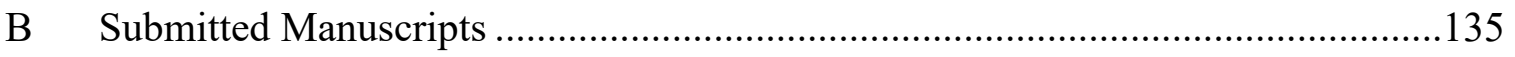

B.1 Home-Based Exercise with Blood Flow Restriction to Restore Limb

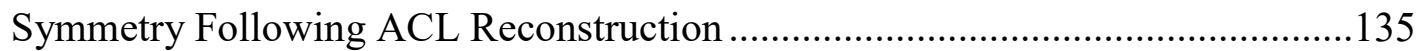

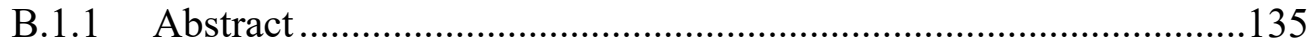


B.1.2 Introduction ................................................................136

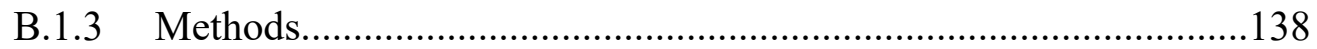

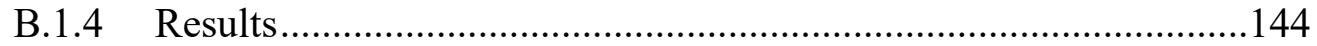

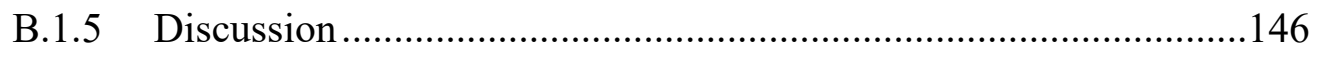

B.2 Home-Based Exercise with Blood Flow Restriction to Improve Quadriceps and Physical Function after Total Knee Arthroplasty: A Case Report ...................167

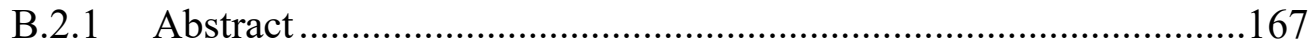

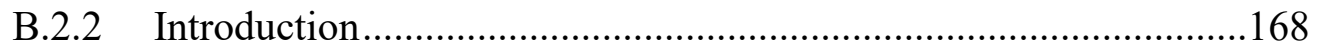

B.2.3 Case Description ...................................................................169

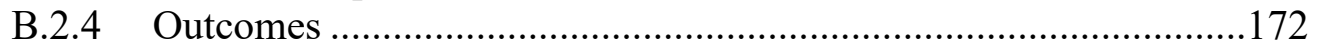

B.2.5 Discussion .......................................................................... 173

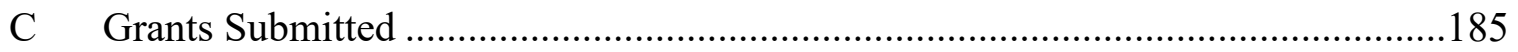

C.1 Blue Cross Blue Shield of Michigan Foundation.......................................185

C.2 Michigan Space Grant Consortium ........................................................189

C.3 American College of Sports Medicine Student Award Program .................195

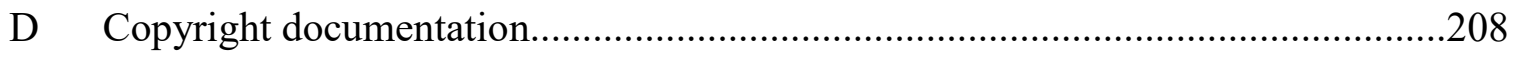




\section{List of figures}

Figure 1: A summary of the FITT principle for resistance and aerobic training .............16

Figure 2: Conceptual model of the physiological responses and.................................18

Figure 3: Time course of alterations in absolute brachial artery blood flow ..................41

Figure 4: Time course of alterations in relative brachial artery blood flow ...................42

Figure 5: Time course of alterations in tissue saturation index (A) across.....................43

Figure 6: Time course of alterations in concentration of deoxyhemoglobin (A) ...........44

Figure 7: Alterations in mean arterial pressure (MAP) by time. ..................................45

Figure 8: Overview of experimental cycling protocol. Base, Cuff, E, R,....................69

Figure 9: Alterations in superficial femoral artery blood flow across ...........................70

Figure 10: Changes in tissue saturation index (A) and concentrations of ......................71

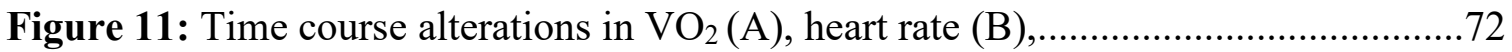

Figure 12: Maximal voluntary isometric contraction torque (MVIC)...........................73

Figure 13: Overview of aerobic and resistance training protocol...............................91

Figure 14: Mean weekly heart rate measured during exercise (A) .............................93

Figure 15: Pre- to post-training changes in lower limb lean mass (A) ..........................94

Figure 16: Pre- to post-training changes in isometric strength (A) ............................95

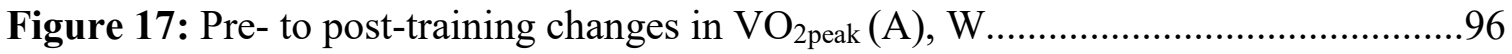




\section{List of tables}

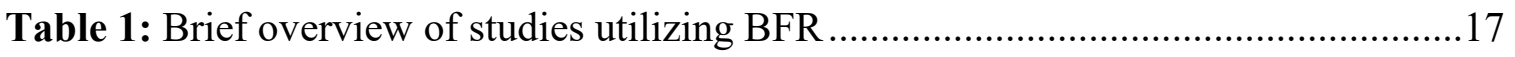

Table 2: Participant demographic and anthropometric characteristics $(\mathrm{n}=10) \ldots \ldots \ldots \ldots . . .40$

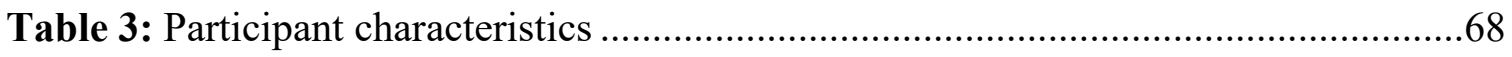

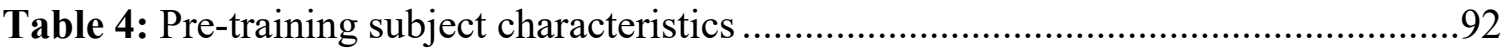




\section{Abstract}

Exercise with blood flow restriction (BFR) allows healthy, clinical, and athletic populations to improve their strength and exercise capacity. The main advantages exercise with BFR has over traditional training are: 1) increases in muscle size, strength, and exercise capacity are elicited at low training loads, 2) these adaptations occur faster with blood flow restriction, 3) increases in muscle size and strength can be stimulated during both resistance and aerobic exercise. Currently, there are no standardized guidelines for exercise with BFR. I used a variety of experimental techniques including ultrasound, near-infrared spectroscopy, expired air analysis, electrical stimulation, and dual-energy X-ray absorptiometry to investigate how cuff pressure and as well as the type of exercise alter acute and chronic responses to exercise with BFR. I was the first to report changes in blood flow during resistance exercise with BFR, and before and after aerobic exercise with BFR. Additionally, I am the first to directly report differences in muscle size, strength, and exercise capacity following aerobic or resistance training with BFR. Overall, I found that the relative reduction in blood flow measured prior to exercise is maintained during exercise. Additionally, I found that moderate cuff pressures of $\sim 60 \%$ of limb occlusion pressure increase metabolic stress without completely occluding blood flow, and therefore is an adequate pressure for both aerobic and resistance exercise with BFR. Finally, I found that aerobic exercise with BFR may be more favorable than resistance exercise because it results in similar increases in muscle size and strength, but at a lower ratings of perceived effort and pain. Taken together, these studies will enable 
researchers, clinicians, and coaches to more effectively prescribe exercise with BFR to improve muscle size, strength and exercise capacity. 


\section{Evidenced based guidelines for exercise with BFR}

Currently, $80 \%$ of US adults do not meet the necessary guidelines for aerobic and resistance training [1] . Both aerobic and resistance training have a profound impact on physical and mental health including a delay in all-cause mortality and a reduced risk for cardiovascular disease, stroke, diabetes, and some cancers [2]. The American College of Sports Medicine outlines effective exercise prescription as the improvement of health related outcomes. The dose of physical activity is defined using the FITT principle. FITT is an acronym for frequency, intensity, time and type of exercise. The proper dose of aerobic and resistance exercise for health benefits is outlined in Figure 1.

Traditional aerobic and resistance training is not always possible for clinical populations where the high training intensities are contraindicated or impossible [3]. These populations include people post-surgery, where high loads may compromise the repaired tissue [4]. In addition, those with neurological disorders such as Cerebral Palsy, where the patient is not able to fully activate the target muscle [3]. Finally, older, deconditioned adults may not be able to reach the target intensity [5]. Aerobic or resistance exercise with blood flow restriction (BFR) is emerging as an effective alternative option to build strength in healthy [6-9], clinical [4, 5, 10-13], and athletic [14-16] populations (Table 1). This exercise modality involves the use of mechanical compression of the limb, usually with a pressurized cuff or tourniquet, used in combination with much lower loads than traditional training [17]. Although the exact mechanism for increased muscle function is unknown, some proposed mechanisms 
include accelerated fatigue leading to preferential type II fiber recruitment [18], augmented metabolite accumulation and local hypoxia [19], and cell swelling [20]. Moreover, there may be an endocrine response as exercise with BFR increases serum growth hormone to a greater extent than traditional high load resistance training [21]. It is thought that this increase in growth hormone secretion occurs through the stimulation of chemoreceptors due to decreases in muscle perfusion [22] (Figure 2).

The main advantages exercise with BFR has over traditional training are: 1) an increase in muscle size and strength elicited at low training loads, 2) these adaptations occur faster with blood flow restriction, 3) increases in muscle size and strength can be stimulated during both resistance and aerobic exercise. Despite the numerous benefits, there are no standardized recommendations for the application of BFR during exercise [17]. Injuries are rare, but improper application of BFR could result in detrimental side effects. Therefore, appropriate evidence-based guidelines for exercise prescription are warranted.

\subsection{Frequency}

Exercise training with BFR has been performed anywhere from two sessions per week, to two sessions per day [23]. An analysis on the use of exercise with BFR reported that $\sim 40 \%$ of practitioners perform BFR exercise 1-2 days per week, whereas $\sim 10 \%$ perform exercise with BFR twice daily. The majority of users $\sim 75 \%$ practiced BFR between 1-4 days per week [23]. Low-load resistance exercise with BFR can be completed more frequently than more traditional resistance training programs because 
exercise with BFR results in no prolonged decrement in muscle function, no prolonged cell swelling, no elevation in blood biomarkers indicative of muscle damage, and low ratings of muscle soreness [24]. To the best of my knowledge there was only been one study the directly assessed training frequency. Abe and colleagues [25] found that oncedaily walking training with BFR resulted in about half of the changes in muscle size and strength as a twice-daily training regimen (muscle size: 2.4 vs. $7 \%$ and muscle strength: 4 vs $9 \%$ respectively). Therefore, the authors suggested that training with BFR is dependent on frequency of training. Additionally, a meta-analysis from Loenneke and colleagues [26] revealed that increases in muscle size and strength are greater when exercise with BFR is performed 2-3 days per week compared to 4-5 days per week (effect size: 1.25 vs 0.53). These results could be explained by an overtraining response. More research is needed in order to elucidate the proper training frequency for exercise with BFR.

\subsection{Intensity}

The primary benefit of performing exercise with BFR is that exercise training is effective at much lower training intensities than traditional exercise. Currently, the majority of practitioners are performing exercise training with BFR at between $20-40 \%$ of one repetition max [23]. Training intensities of as little as $\sim 10 \%$ maximal voluntary isometric contractions have resulted in significant increases in muscle size and strength [27]. Walking, bodyweight squats, and resistance band exercises with BFR have all been used to effectively increase muscle size and strength $[4,6,28]$. Acute studies assessing the effect of exercise intensity have demonstrated that increasing the load from $20 \%$ to 
$30 \%$ one repetition max increases muscle activation, increases concentrations of whole blood lactate [29]. Suga and colleagues [30] also suggest that exercise intensities of $>$ $30 \%$ of one repetitions max would be necessary to create similar intramuscular metabolic stress as high-load resistance training. Cook and colleagues [31], however, reported knee extension repetitions until failure at $20 \%$ of one repetition max resulted in greater muscle fatigue, and possibly greater increases in plasma growth hormone then repetitions until failure at $40 \%$ one repetition max. Lixandrao and colleagues [32] reported increasing the training intensity from $20 \%$ to $40 \%$ of 1 repetition max resulted in greater gains in muscle cross sectional area (effect size: $0.74,2.54$, respectively), with similar increases muscular strength ( $\sim 12 \%)$. Additionally, Patterson and Ferguson [33] have suggested that increasing exercise training intensity from $25 \% 1$ repetition max to $50 \%$ of 1 repetition

max leads to greater increases in muscle strength. Therefore, increasing exercise intensity will likely result in greater gains in muscle size and strength.

\subsection{Time}

Currently, $45 \%$ of practitioners are performing resistance training with BFR in a repetition scheme in which 30 repetitions is performed in the first set, followed by three to four subsequent sets of 15 repetitions, and $32 \%$ of practitioners are performing repetitions until failure [23]. There are no studies directly comparing these two common repetition schemes. Between $15.5-60$ seconds of rest are commonly used between sets. In a meta-analysis, Loenneke and colleagues [26] found significant greater gains in muscle strength during 30 second rest period compare to 60 second rest periods (effect 
size: 1.22 vs. 0.25 ), however, the only studies utilizing 60 second rest periods used a different mode of exercise - walking, and therefore further research is needed regarding the appropriate repetition scheme and duration of rest intervals.

During aerobic exercise with blood flow restriction, it is common for practitioners to perform either multiple 1 to 3 minute walking or cycling intervals with $15-60$ second rest periods between intervals $(\sim 40 \%)$, or continuous aerobic exercise (no rest periods) greater than 5 minutes $(\sim 20 \%$; [23]). Corvino and colleagues [34] reported a greater reduction in tissue perfusion during intermittent cycling with BFR, this reduction was similar to that of high intensity cycling without BFR. Additionally, concentrations of whole blood lactate was greater and ratings of perceived exertion were lower than continuous cycling with BFR [34]. Therefore, the authors suggest that intermittent cycling with BFR may be more advantageous than continuous cycling with BFR. Data from study two supports this conclusion. While intermittent cycling seems to be more advantageous then continuous cycling, little is known about the appropriate duration of the intervals and rest periods.

\subsection{Type}

Exercise with BFR has been effectively combined with different modes of resistance and aerobic exercise to increase muscle size and strength. These modes include single and multi- joint upper and lower body exercise, free weight exercises, and machine-based exercises for resistance training. For aerobic exercise cycling, walking, running, and swimming with BFR have all been practiced [23]. Exercise with BFR is 
most commonly performed using resistance exercise [23]. Although not directly analyzed, previous researchers suggest resistance training with BFR would result in significantly greater increases in strength and hypertrophy compared to aerobic training with BFR [17, 26, 35]. Loenneke et al. reported an effect size of 1.08 for resistance exercise and 0.39 for walking with BFR [26]. However, aerobic exercise with BFR has also been reported to increase aerobic capacity $\left(\mathrm{VO}_{2 \max }\right.$, time until exhaustion, and onset of blood lactate) [10, 36-38]. Additionally, recent evidence also suggests that aerobic exercise with BFR elicits a lower cardiovascular response (e.g., heart rate, cardiac output, mean arterial pressure) [35] and perceived effort than resistance exercise with BFR [35, 39]. Therefore, aerobic exercise with BFR is not only a two for one activity that increase both muscle function and aerobic capacity, but is also more tolerable than resistance training. To date, there has not been a comparison of training adaptations following aerobic and resistance training within equivalent matched groups. This would allow clinicians and practitioners to make a more informed decision of the type of exercise to perform with BFR.

\subsection{Type of Cuff}

Blood flow restriction has been applied using handheld inflatable pumps, automatic inflatable pumps, knee wraps, Kaatsu training devices, and elastic tourniquets [23]. Studies assessing different cuff types have been limited to assessing the differences between elastic and non-elastic (nylon) pressure cuffs. Loenneke and colleagues [40] reported no significant differences between repetitions performed, RPE, or discomfort 
between the elastic and nylon cuffs. In a separate study, Loenneke and colleagues [41] also reported similar reductions in blood flow at rest between elastic and nylon cuffs. Additionally, Rossow and colleagues [42] assessed differences in acute cardiovascular responses to using a $5 \mathrm{~cm}$ elastic cuff and $13.5 \mathrm{~cm}$ non-elastic cuff at the same absolute pressure (130\% of systolic blood pressure). These authors reported wider, non-elastic cuffs increased cardiovascular and perceptual responses compared to narrower, elastic cuffs. These responses occurred despite less volume performed using the wide cuffs. This finding is likely due to the due to differences in cuff width more so than cuff type.

The width of the pressure cuff is also an important factor to consider. A wide range of cuff widths $(3->15 \mathrm{~cm})$ are currently being used to restrict blood flow during exercise [23]. Wider cuff have been shown to increase pain, increase ratings of perceived exertion and decrease training volume when inflated to the same absolute pressure [42]. Wider cuffs also occlude blood flow at a lower absolute pressure than narrower cuffs. Loenneke and colleagues [43] reported that a $5 \mathrm{~cm}$ cuff could not completely lower limb blood flow in some individuals, even when inflated up to $300 \mathrm{mmHg}$. These results suggest that it is easier to reach the target pressure using wider cuffs, especially in the lower body. While the width of the cuff does change the acute response to exercise with BFR when set to the same absolute pressure, both Mouser et al. [44] and Laurentino et al. [45] reported the width of the cuff does not affect acute hemodynamic stress or changes in muscle size and strength respectively, when the same relative pressure is applied. 


\subsection{Cuff Pressure}

A wide range of cuff pressures are used in research and in practice $(<50 \mathrm{mmHg}$ to $>250 \mathrm{mmHg}$ ) [23]. Ideally, the optimal cuff pressure should be set high enough to prevent the return of venous blood flow, but low enough to maintain some level of arterial blood flow [17]. This causes an increase in metabolic stress and an accumulation of fluid within the limb, both of which are thought to be responsible for training adaptions following exercise with BFR $[3,20]$. It is common for researchers and practitioners to use absolute cuff pressures independent of individual differences, cuff width and cuff size [46]. Failure to account for these differences may lead to either ineffective stimulus for training adaptions if the pressure is too low, or adverse sideaffects and/or reduction in training volume if the pressure is too high [3, 47]. It is therefore extremely important that the cuff pressure used be individualized to the participant, however $44 \%$ of practitioners are currently not accounting for individual differences [23].

Researchers have used various methods to individualize cuff pressure. A Doppler ultrasound has been used to directly measure limb arterial occlusion pressure at rest and use a percent of that measurement for exercise with BFR [45]. It is common for researchers to then apply between $40 \%-90 \%$ of this occlusion pressure for exercise training with BFR $[32,48,49]$. This range of pressure was found to result in similar decreases in brachial blood flow at rest [49] and similar increases in muscular size and strength following training $[32,48]$. It should be noted that within this range of relative 
pressures both ratings of perceived exertion, and discomfort increase as relative pressure increases [47, 48]. Additionally, there is evidence to support cuff pressures above $60 \%$ of limb occlusion pressure would not further augment training adaptions [29]. It is important to note that the measurement of limb occlusion pressure is influenced by body position $[50,51]$, therefore this measurement should be taken in the body position in which exercise with BFR will be performed. The measurement of limb occlusion pressure with a Doppler ultrasound, however, has little practical relevance due to the high cost and limited availability of the equipment [52].

Several investigators have applied individual cuff pressure based on brachial systolic blood pressure. The method is controversial as studies have reported the relationship between systolic blood pressure and limb occlusion pressure to be moderate $(\mathrm{r}=0.56,[53]$, poor $(\mathrm{r}=0.49 ;[54])$ or absent $(\mathrm{r}=0.05$ [55]; [43]). Loenneke and colleagues [56] have reported brachial systolic blood pressure could be taken into account for exercise with BFR in the upper limbs, and ankle systolic blood pressure, was found in this study to be a significant predictor of limb occlusion pressure in the lower limbs [43]. Brachial diastolic blood pressure may be predictive of limb occlusion pressure because of its link with peripheral resistance provided limb composition or subcutaneous adipose thickness is also considered $[54,56]$.

Other researchers have used measurements of limb circumference to individualize cuff pressure. Loenneke and colleagues [43] have reported $64 \%$, and $31 \%$ of variance in arterial occlusion pressure for wide $(13.5 \mathrm{~cm})$ and narrow $(5 \mathrm{~cm})$ cuffs, respectively, is explained by limb circumference, and recommend the following for thigh circumferences 
$<45-50 \mathrm{~cm}=120 \mathrm{mmHg}, 50-55 \mathrm{~cm}=150 \mathrm{mmHg}, 56-59 \mathrm{~cm}=180 \mathrm{mmHg}$, and $>60 \mathrm{~cm}$ $=210 \mathrm{mmHg}$. These pressures are thought to estimate $\sim 60 \%$ of limb occlusion pressure when using a $5 \mathrm{~cm}$ nylon pneumatic cuff in the lower limb. Similar recommendations have not been made for different size cuffs or for the upper body. However, Hunt and colleagues [54] report limb circumference has a limited impact on cuff pressure required for partial blood flow restriction.

If the cuff pressure cannot be accurately measured (e.g., when knee wraps and elastic tourniquets are used to restrict blood flow), Wilson and colleagues [52] suggest the wrapping the legs at level of 7 out of 10 , where " 0 " represents no pressure, and 10 represent "intense pressure with pain." This tightness was shown to maintain arterial blood flow and occlude venous return at rest. While this method does have practical applications, basing the pressure on perceived discomfort will likely result in large differences in relative restriction of blood flow among individuals due to differences in perception of pain.

Finally, is important to consider if the cuff pressure should be maintained or release during inter-set rest periods. Suga and colleagues [30], reported similar increases in metabolite accumulation to high load resistance training only when the pressure was maintain during the inter-set rest periods. Theoretically, if the cuff pressure is maintained during the inter-set rest periods, venous outflow will be occluded and metabolite clearance will be drastically diminished. Moreover, additional fluid pooling in the limb during rest will increase cell swelling. Both of which are thought to be important mechanisms for adaptations following training [17]. Maintaining the cuff pressure during 
rest does, however, result in more muscle pain and discomfort throughout training when compared to no cuff pressure during the inter-set rest period [57]. Anecdotal feedback from our participants suggests the pain experienced during the inter-set rest when the cuff remains inflated is greater than the pain experienced during exercise. Additionally, increases in muscle activation [58] and in muscle fatigue [31, 57] have been found to be similar following a bout of either continuously, or intermittently applied pressure. Fitschen and colleagues [57] reported that applying either continuous or intermittent pressure resulted in similar increases in muscular strength. However, the effect size reported in this study was minimal (effect size: 0.1 ). More research is needed to determine whether or not the cuff pressure should be maintained during the inter-set rest periods.

\subsection{Summary}

The purpose of this review is to summarize current practices for exercise with BFR, as well as outline safe and effect doses of exercise with BFR. Practitioners of exercise with BFR are currently using a wide range of frequencies ( $2 x /$ day to $2 x /$ week), intensities (10-90\% of 1 repetition max), times ( 1 minutes) to $>$ (5 minutes), and types of exercise with BFR (resistance vs. aerobic). Practitioners are also using many different cuffs of different widths $(<3 \mathrm{~cm}->15 \mathrm{~cm})$, inflated to a wide range of pressures $(<50 \mathrm{mmHg}$ to $>250 \mathrm{~mm} \mathrm{Hg}$; [23]. Although injuries from exercise with BFR are rare, Nakajima and colleagues [59] reported side effects including bruising and numbness, and in rare cases venous thrombus $(0.055 \%)$, pulmonary embolism $(0.008 \%)$ and rhabdomyolysis 
$(0.008 \%)$. These side effects may be reduced by careful consideration of the dose of exercise with BFR.

In practice it may be beneficial to limit exercise sessions to 2-3 days per week [26]. A major benefit of exercise with BFR is that increases in muscle size and strength are stimulated at lower training loads. While increases in intensity have been shown to result in greater increases in muscular strength, practitioners should consider individual abilities when selecting an appropriate intensity. In general, intensities between 30-50\% of 1 repetition max are effective for resistance training. Little information is currently known about the appropriate time for aerobic and resistance training with BFR. While the optimal protocol for resistance training is unknown, a common repetition scheme used in training is the $30-15-15-15$ repetition scheme. Interestingly, the volume performed using this repetition scheme at $30 \%$ of 1 repetition max is similar to that of high intensity resistance exercise (e.g. four sets of five repetitions at $80 \%$ of 1 repetition max) [16]. Additionally, for aerobic exercise, performing multiple 1-3 minute intervals with 30-60 seconds rest between intervals appears to be more beneficial than continuous training [34]. In practice resistance training with BFR is more common than aerobic training with BFR, and there may be greater gains in muscle size and strength when performing resistance training. However, this has not been directly tested.

Measures should be taken to individualize cuff pressure. Although imperfect, using limb circumference could be used to do this [60]. Loenneke and colleagues [43] outlined guidelines for doing this with a $5 \mathrm{~cm}$ cuff. Practitioners should use a lower pressure than listed if wider cuffs are used. Another option could be to use blood pressure 
measured in the anterior tibial or popliteal artery for lower body exercise, or blood pressure measured in the brachial artery for upper body exercise $[43,54]$. If using a type of cuff that does not allow for accurate measure of cuff pressure, care should be taken to ensure arterial blood flow is not occluded. To do this practitioners could palpate or auscultate an artery below the cuff to ensure blood flow is maintained, although this method has not been tested. Finally, maintaining the cuff pressure during the inter-set rest period should theoretically increase training adaptations [17], however, this has not yet been reported.

\subsection{Gaps and Future Directions}

Clearly more information is needed to determine the optimal dose of exercise with BFR., Researchers need to clearly identify their subject populations (e.g. blood pressures, limb circumference, training status, limb subcutaneous fat thickness, etc.) and BFR protocols (e.g. cuff type, cuff width, and both absolute and relative cuff pressures). This should allow for more careful replication in research and in practice.

\subsubsection{Study 1}

Theoretically, cuff pressure should maximize metabolic stress without completely occluding arterial blood flow blood flow during exercise [17]. While researchers have measured blood flow before and after training, there are no previous reports documenting changes in blood flow during exercise with BFR. The purpose of the first experiment was to evaluate changes in arterial blood flow, tissue perfusion, and cardiovascular responses 
before, during, and after exercise with BFR across various pressures. Participants performed rhythmic handgrip exercise under cuff pressures ranging from $0-120 \%$ of limb occlusion pressure. We hypothesized that during exercise, blood flow would increase, and increasing cuff pressure will reduce blood flow during exercise.

\subsubsection{Study 2}

BFR during walking and cycling not only increases muscle size and strength but also improves exercise capacity $(1,3-5,14,16,55)$. The purpose of the second experiment was to evaluate blood flow and tissue perfusion during intermittent trials of cycling with BFR. A secondary purpose was to assess changes in muscle function (i.e. central and peripheral fatigue) following trials of cycling with BFR. Participants completed four trials of cycling during in which we measured blood flow and tissue perfusion. Following each trial we measured changes in maximal voluntary torque and central and peripheral factors related to fatigue. We hypothesized that as pressure increases blood flow, tissue perfusion and maximal voluntary torque will decrease.

\subsubsection{Study 3}

Both aerobic and resistance training increase muscle function. Researchers have suggested that resistance training with BFR would lead to greater increases in muscle size and strength than aerobic training with BFR. However only aerobic training has been shown to increase exercise capacity. Additionally, aerobic exercise with BFR has a lower cardiovascular response (reduced heart rate, cardiac output, and mean arterial pressure) than resistance training with BFR (44). It is unclear which type of exercise should be 
used with BFR. The purpose of this study was to compare chronic adaptations in muscular function and exercise capacity following 6 weeks of aerobic training or resistance training with BFR. Pre- to post-training measures of muscular size, strength and exercise capacity was be assessed. We hypothesized that both resistance training with BFR and aerobic training with BFR will increase muscle function, but only aerobic training will increase exercise capacity. 


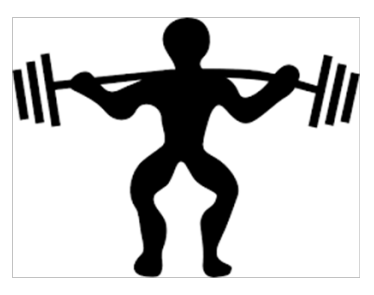

Resistance Training

- FITT Principle

- Frequency: 2-3x/week

- Intensity: $60-80 \% 1$ repetition max

- Time: $30-60$ minutes

- Type: weight training

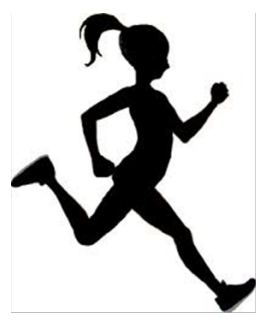

Aerobic Training

- FITT Principle

- Frequency: 3-5x/week

- Intensity: $50-80 \% \mathrm{VO}_{2 \max }$

- Time: 20-60 minutes

- Type: aerobic activity

Figure 1: A summary of the FITT principle for resistance and aerobic training 
Table 1: Brief overview of studies utilizing BFR

\begin{tabular}{|c|c|c|c|c|c|}
\hline Study & Population & Exercise & $\begin{array}{l}\text { Cuff } \\
\text { pressure }\end{array}$ & Duration & $\begin{array}{l}\text { Outcome } \\
\text { measure }\end{array}$ \\
\hline $\begin{array}{l}\text { Takarada } \\
\text { et al. } \\
(2002)\end{array}$ & $\begin{array}{l}\text { Rugby } \\
\text { players }\end{array}$ & $\begin{array}{l}\text { Knee } \\
\text { extension }\end{array}$ & $\begin{array}{l}200 \\
\mathrm{mmHg}\end{array}$ & 8 weeks & $\begin{array}{l}\uparrow 15 \% \text { size, } \uparrow 14 \% \\
\text { strength, } \uparrow 2-4 \% \\
\text { endurance }\end{array}$ \\
\hline $\begin{array}{l}\text { Abe et } \\
\text { al. (2005) }\end{array}$ & $\begin{array}{l}\text { Collegiate } \\
\text { track and field } \\
\text { athletes }\end{array}$ & $\begin{array}{l}\text { Squat and leg } \\
\text { curl }\end{array}$ & $\begin{array}{l}160-240 \\
\mathrm{mmHg}\end{array}$ & 8 days & $\begin{array}{l}\uparrow 4-6 \% \text { size, } \\
\uparrow 10 \% \text { strength }\end{array}$ \\
\hline $\begin{array}{l}\text { Park et } \\
\text { al. }(2010\end{array}$ & $\begin{array}{l}\text { Collegiate } \\
\text { basketball } \\
\text { athletes }\end{array}$ & $\begin{array}{l}\text { Treadmill } \\
\text { walking }\end{array}$ & $\begin{array}{l}120-160 \\
\mathrm{mmHg}\end{array}$ & 2 weeks & $\begin{array}{l}\uparrow 12 \% \text { exercise } \\
\text { capacity }\end{array}$ \\
\hline $\begin{array}{l}\text { Fujita et } \\
\text { al. (2008) }\end{array}$ & $\begin{array}{l}\text { Young } \\
\text { healthy }\end{array}$ & $\begin{array}{l}\text { Knee } \\
\text { extension }\end{array}$ & $\begin{array}{l}200 \\
\mathrm{mmHg}\end{array}$ & 6 days & $\begin{array}{l}\uparrow 2-4 \% \text { size, } \uparrow 7 \% \\
\text { strength }\end{array}$ \\
\hline $\begin{array}{l}\text { Abe et } \\
\text { al. (2006) }\end{array}$ & $\begin{array}{l}\text { Young } \\
\text { healthy }\end{array}$ & $\begin{array}{l}\text { Treadmill } \\
\text { walking }\end{array}$ & $\begin{array}{l}200 \\
\mathrm{mmHg}\end{array}$ & 3 weeks & $\begin{array}{l}\uparrow 4-7 \% \text { size, } \uparrow 8- \\
10 \% \text { strength }\end{array}$ \\
\hline $\begin{array}{l}\text { Natsume } \\
\text { et al. } \\
\text { (2015) }\end{array}$ & $\begin{array}{l}\text { Young } \\
\text { healthy }\end{array}$ & $\begin{array}{l}\text { BFR with } \\
\text { neuromuscular } \\
\text { electric } \\
\text { stimulation }\end{array}$ & $\begin{array}{l}140-200 \\
\mathrm{mmHg}\end{array}$ & 2 weeks & $\begin{array}{l}\uparrow 4 \% \text { size, } \uparrow 14 \% \\
\text { strength }\end{array}$ \\
\hline $\begin{array}{l}\text { Abe et } \\
\text { al. }(2010)\end{array}$ & Elderly & $\begin{array}{l}\text { Treadmill } \\
\text { walking }\end{array}$ & $\begin{array}{l}160-200 \\
\mathrm{mmHg}\end{array}$ & 6 weeks & $\begin{array}{l}\uparrow 6-10 \% \text { size, } \uparrow 7- \\
16 \% \text { strength }\end{array}$ \\
\hline $\begin{array}{l}\text { Yasuda } \\
\text { et al. } \\
\text { (2015) }\end{array}$ & Elderly & $\begin{array}{l}\text { Arm curls } \\
\text { triceps pull- } \\
\text { down }\end{array}$ & $\begin{array}{l}180-270 \\
\mathrm{mmHg}\end{array}$ & $\begin{array}{l}12 \\
\text { weeks }\end{array}$ & $\begin{array}{l}\uparrow 17-18 \% \text { size, } \\
\uparrow 8-16 \% \text { strength }\end{array}$ \\
\hline $\begin{array}{l}\text { Ohta et } \\
\text { al. (2003) }\end{array}$ & $\begin{array}{l}\mathrm{ACL} \\
\text { reconstruction }\end{array}$ & $\begin{array}{l}\text { Lower limb } \\
\text { exercises }\end{array}$ & $\begin{array}{l}180 \\
\mathrm{mmHg}\end{array}$ & $\begin{array}{l}16 \\
\text { weeks }\end{array}$ & $\begin{array}{l}\downarrow 8-13 \% \text { strength } \\
\text { less than control }\end{array}$ \\
\hline $\begin{array}{l}\text { Takarada } \\
\text { et al. } \\
\text { (2000) }\end{array}$ & $\begin{array}{l}\mathrm{ACL} \\
\text { reconstruction }\end{array}$ & $\begin{array}{l}\text { Occlusion and } \\
\text { reperfusion }\end{array}$ & $\begin{array}{l}200-260 \\
\mathrm{mmHg}\end{array}$ & 11 days & $\begin{array}{l}\downarrow 10 \% \text { size less } \\
\text { than control }\end{array}$ \\
\hline $\begin{array}{l}\text { Bryk et } \\
\text { al. (2016) }\end{array}$ & $\begin{array}{l}\text { Knee } \\
\text { osteoarthritis }\end{array}$ & $\begin{array}{l}\text { Knee } \\
\text { extension }\end{array}$ & $\begin{array}{l}200 \\
\mathrm{mmHg}\end{array}$ & 6 weeks & $\begin{array}{l}\uparrow 42 \% \text { strength, } \\
\downarrow \text { pain }\end{array}$ \\
\hline $\begin{array}{l}\text { Gaunder } \\
\text { et al. } \\
(2017)\end{array}$ & $\begin{array}{l}\text { Total knee } \\
\text { arthroplasty }\end{array}$ & $\begin{array}{l}\text { Knee } \\
\text { extension, leg } \\
\text { press, reverse } \\
\text { press }\end{array}$ & $\begin{array}{l}100-150 \\
\mathrm{mmHg}\end{array}$ & 8 weeks & $\begin{array}{l}\uparrow 57-359 \% \\
\text { strength }\end{array}$ \\
\hline
\end{tabular}




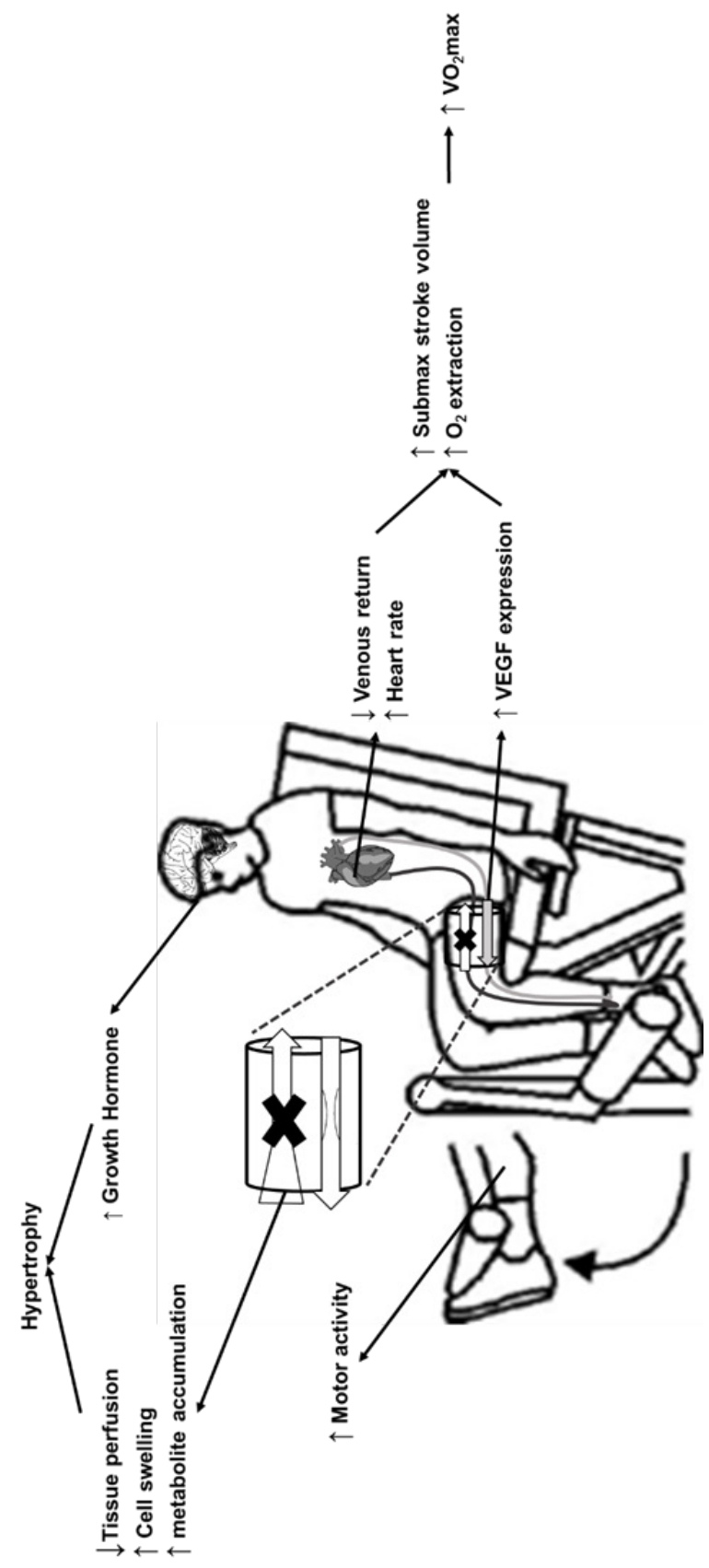

Figure 2: Conceptual model of the physiological responses and adaptations to exercise with blood flow restriction. 


\section{Limb Blood Flow and Tissue Perfusion during Exercise with Blood Flow Restriction}

\subsection{Introduction}

Resistance exercise is widely recommended to restore function following injury, maintain health across the lifespan, and improve athletic performance. In general, performing resistance exercise at $65-70 \%$ of one repetition maximum (RM) 2-3 times per week for 8 weeks is sufficient to increase muscle size and strength [2]. This type of exercise, however, is not always possible because high training loads are often contraindicated or impossible for some populations (e.g., older adults, individuals with orthopedic limitations). Alternatively, implementation of resistance exercise with blood flow restriction (BFR) is emerging as an effective option to build muscle and improve strength in healthy, clinical, and athletic populations [17]. This exercise modality involves the use of mechanical compression of the limb to occlude blood flow, usually with a pressurized cuff or tourniquet, used in combination with much lower loads than traditional resistance training (e.g., $20 \%$ vs. $70 \%$ of $1 \mathrm{RM}$ ) [17]. The main advantage of exercise with BFR is that increases in muscle size and strength are elicited at low training loads which is clinically relevant when high loads are contraindicated (for a review see Hughes et al. 2017).

To date, there are no standardized recommendations for the proper application of cuff pressure during exercise with BFR. Theoretically, cuff pressure should be set high enough to prevent the return of venous blood flow, but low enough to maintain some level of arterial blood flow [17]. Cuff pressure should also maximize metabolic stress 
$[61,62]$ without completely occluding arterial blood flow and inducing excessive discomfort. Several previous investigators have measured blood flow at rest with BFR $[44,49,54,63]$. Specifically, brachial and femoral artery blood flow decreases linearly with increasing cuff pressure between 80-120 and 100-250 mm Hg, respectively [54, 63]. When cuff pressure is set relative to individual limb occlusion pressure (LOP), a nonlinear decrease occurs with minimal changes in blood flow from 50-90\% of LOP [44, 49]. However, it is unclear if the cuff-induced changes in blood flow measured at rest translates to similar hemodynamic changes during exercise. Several factors including increase in blood pressure, activation of the skeletal muscle pump, and change in limb position $[50,51]$ during exercise may reduce the effectiveness of the cuff at restricting blood flow. Previous investigators have used near-infrared spectroscopy to quantify tissue oxygenation during exercise with BFR [34, 61, 64], which has provided indirect insight into the level of blood flow and metabolic stress associated with BFR cuff pressures. To our knowledge, there are no reports documenting changes in blood flow and tissue perfusion during exercise with BFR.

Understanding the extent to which cuff pressure alters blood flow and tissue perfusion during exercise with BFR would have important implications for exercise prescription and training. Most notably, high cuff pressures that completely occlude arterial blood flow could cause harmful side effects including adverse cardiovascular responses, blood clotting, and muscle/nerve damage [3]. Accordingly, the purpose of this study was to evaluate changes in arterial blood flow and tissue perfusion before, during, and after rhythmic handgrip exercise with BFR across a range of cuff pressures. We 
hypothesized that BFR-induced reductions in arterial blood flow during exercise would be less than those reductions during rest. We also hypothesized that application of moderate pressure, based on LOP, would elicit considerable metabolic stress (as quantified by reductions in tissue saturation index and increases in deoxygenated hemoglobin concentration) without completely occluding arterial blood flow.

\subsection{Methods}

\subsubsection{Participants}

Ten active men between 18-44 yrs volunteered to participate in this study (demographic and anthropometric characteristics reported in Table 1). All participants self-reported that they performed physical activity at a moderate intensity at least 150 $\mathrm{min} /$ week, which is consistent with the American College of Sports Medicine guidelines [2]. However, participants did not perform exercise with BFR on a regular basis. Participants were excluded from the study if they used nicotine products, had diabetes, or had any cardiopulmonary disorders. Following the initial screening, participants were informed of the purpose of the study, the risks involved, and gave informed written consent. This study was approved by the Institutional Review Boards at Michigan Technological University and Kent State University. Finally, given the effect size calculated from partial eta squared (0.823), an alpha level of 0.05 , and a sample of 10 participants, the statistical power was calculated to be 0.99 . 


\subsubsection{Study Design and Overview}

In this investigation, we used a single-group repeated measures design. Participants performed rhythmic handgrip exercise across a range of cuff pressure conditions $(0 \%, 60 \%, 80 \%, 100 \%$, and $120 \%$ of LOP). Our primary outcome variable was brachial artery blood flow and secondary outcome variables included tissue saturation index and deoxyhemoglobin concentration. These variables were selected because level of blood flow restriction and metabolic stress are factors that likely contribute to the robust increases in muscle size and strength with BFR exercise training $[34,65]$. Primary and secondary variables were assessed before, during, and after exercise. With this experimental design, we aimed to determine the extent to which cuff pressure alters blood flow and tissue perfusion during exercise with BFR.

Participants reported to the laboratory in a resting condition, not having consumed alcohol or participated in vigorous exercise in the previous $24 \mathrm{~h}$. Participants were asked to lay supine on an examination table for 10 min prior to testing and remained in this position for the duration of the experimental protocol. Resting heart rate, mean arterial pressure, and cardiac output were monitored to ensure normal physiological ranges. Resting brachial artery blood flow was monitored for 1 min and then LOP was determined. Relative pressures of $0 \%, 60 \%, 80 \%, 100 \%$, and $120 \%$ of LOP were calculated and the order of pressures was randomized. Participants rested for 5 min for physiological values to return to baseline. Participants then performed rhythmic handgrip contractions at each pressure condition, with 5 min of rest between conditions. All testing was performed in a controlled thermoneutral environment $\left(25^{\circ} \mathrm{C}\right)$. 


\subsubsection{Rhythmic Handgrip Exercise}

Prior to exercise participants performed 3 maximal voluntary contractions (MVC) using a handgrip dynamometer (ADInstruments, Colorado Springs, CO, USA). Handgrip forces were recorded using a data acquisition system (Powerlab 16, ADInstruments, Colorado Springs, CO, USA). The highest MVC was identified and 30\% of the participant's MVC force was calculated and used as the exercise intensity. The cuff was inflated to the desired pressure and was maintained throughout that pressure condition. Pre-exercise brachial artery blood flow was measured for $30 \mathrm{~s}$ with the cuff inflated. Participants then performed 30 handgrip contractions. Participants were instructed to perform one contraction every $2 \mathrm{~s}$ while exercise blood flow was recorded. A metronome was used to help participants match the desired contraction frequency. Force tracings were streamed onto a monitor so that the participants had visual feedback of the force they produced, which enabled them to more accurately reach the target intensity. The cuff remained inflated for $30 \mathrm{~s}$ after exercise to determine if the various cuff pressures had a similar impact on post-exercise blood flow as they did pre-exercise. After the cuff pressure was released, the participant was given 5 min of rest before the next cuff pressure condition.

\subsubsection{Blood Flow Calculation}

Blood velocity $\left(\mathrm{V}_{\text {mean }}\right)$ and vessel diameter $\left(\mathrm{V}_{\mathrm{d}}\right)$ were measured with a Logic 7 ultrasound system (General Electric Medical Systems, Milwaukee, WI) equipped with a linear array transducer operating at an imaging frequency of $12 \mathrm{MHz}$ and Doppler frequency of $5 \mathrm{MHz}$. Doppler pulse wave spectrum and ultrasound images were 
continuously recorded throughout the pre-exercise, exercise, post-exercise period. Vessel diameters were determined by averaging the perpendicular distance between the superficial and deep walls of the brachial artery at three nonconsecutive $\mathrm{R}$ waves during the last $15 \mathrm{~s}$ of the pre-exercise time period. Measurements of $\mathrm{V}_{\text {mean }}$ were obtained with the probe positioned to maintain an insonation angle of $\leq 60^{\circ}$. Mean blood velocity was averaged across $15 \mathrm{~s}$ intervals throughout the protocol. Importantly, blood velocity data obtained during exercise using Doppler ultrasound are reliable [66] which is notable given the complex nature of blood velocity during dynamic muscle contractions. Using pre-exercise mean blood velocity and arterial diameter, blood flow was calculated as Blood Flow $=\mathrm{V}_{\text {mean }} * \pi^{*}\left(\mathrm{~V}_{\mathrm{d}} / 2\right)^{2 *} 60$. Blood flow was averaged over each time period, $30 \mathrm{~s}$ pre-exercise, $60 \mathrm{~s}$ exercise, and $30 \mathrm{~s}$ post-exercise within each cuff pressure. Peak blood flow was determined as the $15 \mathrm{~s}$ intervals with the highest blood flow. Relative blood flow was determined by normalizing blood flow at $60 \%, 80 \%, 100 \%$, and $120 \%$ LOP to the $0 \%$ LOP condition within each time period.

\subsubsection{Limb Occlusion Pressure}

A $10 \mathrm{~cm}$ wide nylon pneumatic cuff (Hokanson, Belleview, WA, USA) was wrapped around the right arm at the most proximal location and pressurized with a rapid cuff inflator (Hokanson, Belleview, WA, USA). The ultrasound probe was positioned distal to the cuff and proximal to the cubital fossa. Limb occlusion pressure was determined by inflating the cuff to $75 \mathrm{mmHg}$, and slowly increasing the pressure until brachial blood velocity reached zero based on the absence of the Doppler spectrum. The minimum pressure required to completely occlude arterial blood flow was recorded as the 
LOP. Finally, previous authors [67] have reported that upper-arm limb occlusion pressure data obtained using Doppler ultrasound are reliable.

\subsubsection{Tissue Perfusion}

A near-infrared spectroscopy (NIRS) system was used to measure tissue oxygen saturation (Oxymon MKIII; Artinis Medical Systems, Einsteinweg, Netherlands). The NIRS sensor was placed on the right forearm approximately $1 / 3$ of the distance from the medial epicondyle of the humerus to the styloid process of the radius and adhered with double-sided tape. The sensor site was prepared by shaving hair, abrading the skin with sandpaper, and removing any oils or contaminants using an alcohol swab prior to sensor placement. Data were recorded at $10 \mathrm{~Hz}$. The concentration of total hemoglobin (THB), oxyhemoglobin (OHB) and deoxyhemoglobin (HHB) was recorded. Tissue saturation index $(\mathrm{TSI})$ was calculated by TSI $(\%)=(\mathrm{OHB} / \mathrm{THB}) * 100$ and averaged over each time period. Changes in deoxyhemoglobin were calculated by assessing the difference between the average value in the last $15 \mathrm{~s}$ of each time period and the baseline values prior to cuff inflation. It is important to note that tissue saturation index and deoxyhemoglobin data obtained during handgrip exercise using NIRS are reliable [68].

\subsubsection{Central Variables}

Beat by beat mean arterial pressure and cardiac output were measured with a Nexfin HD Monitor (Edwards LifeSciences, Irvine CA, USA). Heart rate was determined with the use of a 3-lead ECG running through a BioAmp acquisition box (ADInstruments, Colorado Springs, CO, USA). The finger cuff electrode was placed over 
the middle phalanx of the left, non-exercising hand. These data were also continuously streamed into the power lab 16 and analyzed with Lab chart 8. Each dependent variable was averaged over $15 \mathrm{~s}$ throughout each pressure condition.

\subsubsection{Forearm Muscle Activity}

Forearm muscle activity was assessed using surface electromyography (EMG). Electrodes were placed on the right forearm approximately $1 / 3$ of the distance from the medial epicondyle of the humerus to the styloid process of the radius as recommended by Davis [69]. Disposable self-adhesive electrodes were placed on the muscle in line with the fiber direction. The electrode site placement was prepared in the same manner as the NIRS sensor. EMG data were recorded at 4,000 $\mathrm{Hz}$ and acquired on the data acquisition system described above and analyzed using commercially available software (Lab Chart 8 Pro, ADInstruments, Colorado Springs, CO, USA). The raw EMG signal was bandpass filtered (10-450 Hz) and rectified. Afterward, a root mean squared integration was performed. Integrated software (Peak Analysis, Lab Chart 8 Pro, AD Instruments, Colorado Springs, CO, USA) was used to calculate the mean area under the curve for the first and last 10 contractions for each condition.

\subsubsection{Statistical Analysis}

To test the hypothesis that BFR-induced reductions in arterial blood flow during exercise would be less than those reductions during rest, we used a two-way repeated measures analysis of variance (ANOVA) procedure to evaluate the effects of time (preexercise, exercise, post-exercise) and pressure $(0 \%, 60 \%, 80 \%, 100 \%$, and $120 \%$ of LOP) 
on the primary outcome variable brachial artery blood flow. Note that, changes in both absolute and relative blood flow were assessed. Additionally, to test the hypothesis that application of moderate cuff pressure would elicit considerable metabolic stress, we used separate two-way repeated measures (ANOVA) procedures to evaluate the effects of time and pressure on secondary outcome variables of tissue saturation index and deoxyhemoglobin concentration. Repeated measures ANOVA procedures were also used to assess changes in select cardiovascular response variables (heart rate, mean arterial pressure, cardiac output). When the assumption of sphericity was violated a GreenhouseGeisser correction was used. If there were any significant main effects or interactions identified then subsequent post-hoc tests (Fisher's least significant difference) were used to explore where differences occurred. Paired samples t-tests were used to assess differences in forearm muscle activation for all cuff pressure conditions. Partial eta squared $\left(\eta p^{2}\right)$ values were calculated as a measure of effect sizes with $\eta p^{2} \geq 0.01$ indicating small, $\geq 0.059$ medium, and $\geq 0.138$ large effects, respectively [70]. Statistical procedures were performed using IBM SPSS 24 (Chicago, IL, USA). Data are reported as mean \pm standard deviation and alpha was set to 0.05 .

\subsection{Results}

\subsubsection{Limb Occlusion and Cuff Pressure}

Mean limb occlusion pressure was $130 \pm 12 \mathrm{~mm} \mathrm{Hg}$. Cuff pressures for the $60 \%$, $80 \%, 100 \%$, and $120 \%$ LOP conditions corresponded to $81 \pm 11,108 \pm 15,135 \pm 19$, and $162 \pm 23 \mathrm{~mm} \mathrm{Hg}$, respectively. 


\subsubsection{Blood Flow}

Absolute blood flow kinetics are illustrated in Figure 3A for descriptive purposes. The repeated measures ANOVA procedures revealed significant main effects of pressure $\left(\mathrm{P}<0.01, \eta \mathrm{p}^{2}=0.839\right)$ and time $\left(\mathrm{P}<0.01, \eta \mathrm{p}^{2}=0.823\right)$, as well as a pressure $\mathrm{x}$ time interaction $\left(\mathrm{P}<0.01, \eta \mathrm{p}^{2}=0.725\right)$. At rest prior to exercise, blood flow generally decreased with increased cuff pressure: $0 \%>60 \% \approx 80 \%>100 \% \approx 120 \%$ (Figure 3B). During exercise, blood flow decreased with increased cuff pressure: $0 \%>60 \%>80 \%>$ $100 \%>120 \%$ (Figure 3B). The pressure-blood flow relationships during exercise were also maintained after exercise (Figure 3B). For the $0 \%, 60 \%$, and $80 \%$ LOP conditions, blood flow increased from rest during exercise (all $\mathrm{P}<0.05$, Figure $3 \mathrm{C}$ ). Blood flow decreased after exercise for the $60 \%$ and $80 \%$ LOP conditions (all $\mathrm{P}<0.05$, Figure $3 \mathrm{C}$ ). For the $100 \%$ and $120 \%$ LOP conditions, blood flow did not differ before, during, or after exercise. Note that, pressure-blood flow relationships for peak blood flow followed the same patterns as those for mean blood flow.

Relative blood flow kinetics are illustrated in Figure 4A for descriptive purposes. The repeated measures ANOVA procedures revealed significant main effects of pressure $\left(\mathrm{P}<0.01, \eta \mathrm{p}^{2}=0.927\right)$ and time $\left(\mathrm{P}<0.01, \eta \mathrm{p}^{2}=0.564\right)$, as well as a pressure $\mathrm{x}$ time interaction $\left(\mathrm{P}<0.01, \eta \mathrm{p}^{2}=0.339\right)$. The relative reductions in blood flow before exercise did not differ from those during exercise (Figure 4B). Specifically, blood flow in the $60 \%$ LOP condition was reduced by $22 \pm 3 \%$ before exercise and remained reduced by $22 \pm$ $2 \%$ during exercise (Figure 4 C). Similarly, blood flow in the $80 \%$ LOP condition was reduced by $47 \pm 2 \%$ and $48 \pm 2 \%$ before and during exercise, respectively (Figure $4 \mathrm{C}$ ). 
After exercise blood flow for both the $60 \%$ and $80 \%$ LOP conditions was reduced even further ( $52 \pm 2 \%$ and $71 \pm 1 \%$, respectively, both $\mathrm{P}<0.05$, Figure $4 \mathrm{C})$. In the $100 \%$ and $120 \%$ LOP condition there were no differences in relative blood flow.

\subsubsection{Tissue Perfusion}

Changes in tissue saturation index are illustrated in Figure 5A for descriptive purposes. The repeated measures ANOVA procedures revealed significant main effects of pressure $\left(\mathrm{P}<0.01, \eta \mathrm{p}^{2}=0.682\right)$ and time $\left(\mathrm{P}<0.01, \eta \mathrm{p}^{2}=0.782\right)$, as well as a pressure $\mathrm{x}$ time interaction $\left(\mathrm{P}<0.01, \eta \mathrm{p}^{2}=0.588\right)$. At rest prior to exercise, there were no differences in tissue saturation index with increased pressure. There were trends for the $60 \%, 100 \%$, and $120 \%$ LOP conditions to be lower than $0 \%$ LOP condition $(\mathrm{P}=0.08, \mathrm{P}$ $=0.06, \mathrm{P}=0.07$, respectively). During exercise, tissue saturation index generally decreased with increased pressure: $0 \%>60 \% \approx 80 \%>100 \% \approx 120 \%$ (Figure 5B). After exercise, all pressure conditions were different (all $\mathrm{P}<0.05$ ) except for $80 \%$ and $100 \%$ $(0 \%>60 \%>80 \% \approx 100 \%>120 \%$, Figure 5B). For the $0 \%$ condition, tissue saturation index decreased only after exercise $(\mathrm{P}=0.04$, Figure $5 \mathrm{C})$. For the $60 \%, 80 \%, 100$, and $120 \%$ LOP conditions, tissue saturation index decreased from rest during exercise and decreased further after exercise (all $\mathrm{P}<0.05$, Figure 5C).

Changes in deoxyhemoglobin are illustrated in Figure 6A for descriptive

purposes. The repeated measures ANOVA revealed significant main effects of pressure $\left(\mathrm{P}<0.01, \eta \mathrm{p}^{2}=0.812\right)$ and time $\left(\mathrm{P}<0.01, \eta \mathrm{p}^{2}=0.916\right)$, as well as a pressure $\mathrm{x}$ time interaction $\left(\mathrm{P}<0.01, \eta \mathrm{p}^{2}=0.796\right)$. At rest prior to exercise, deoxyhemoglobin increased with the initial increase in pressure but did not increase at pressures higher than $60 \%$ 
LOP: $0 \%<60 \% \approx 80 \% \approx 100 \% \approx 120 \%$ (Figure 6B). During exercise, deoxyhemoglobin increased with the initial increases in pressure but did not increase at pressures higher than $80 \%$ LOP: $0 \%<60 \%<80 \% \approx 100 \% \approx 120 \%$ (Figure $6 \mathrm{~B}$ ). The pressuredeoxyhemoglobin relationships during exercise were generally maintained after exercise. For the $0 \%$ condition, deoxyhemoglobin increased from rest during exercise and remained elevated after exercise (both $\mathrm{P}<0.05$, Figure $6 \mathrm{C}$ ). For the $60 \%, 80 \%, 100$, and $120 \%$ LOP conditions, deoxyhemoglobin increased from rest during exercise and increased further after exercise (all $\mathrm{P}<0.05$, Figure $6 \mathrm{C}$ ).

\subsubsection{Central Variables}

For cardiac output and heart rate there were no significant main effects for time ( $\mathrm{P}$ $=0.54, \eta p^{2}=0.081, P=0.22, \eta p^{2}=0.145$, respectively $)$ or pressure $\left(P=0.8, \eta p^{2}=0.279\right.$, $\mathrm{P}=0.22, \eta \mathrm{p}^{2}=0.156$, respectively $)$ or pressure $\mathrm{x}$ time interactions $\left(\mathrm{P}=0.07, \eta \mathrm{p}^{2}=0.219\right.$, $\mathrm{P}=0.54, \eta \mathrm{p}^{2}=0.077$, respectively). For mean arterial pressure there was a significant effect of time ( $\left.\mathrm{P}<0.01, \eta \mathrm{p}^{2}=0.625\right)$, but not for cuff pressure $\left(\mathrm{P}=0.59, \eta \mathrm{p}^{2}=0.058\right)$ or the cuff pressure $\mathrm{x}$ time interaction $\left(\mathrm{P}=0.14, \eta \mathrm{p}^{2}=0.151\right)$. Mean arterial pressure increased during exercise in all LOP conditions (all $\mathrm{P}<0.05$ ), but only decreased postexercise in $0 \%$ and $60 \%$ LOP conditions (both $\mathrm{P}<0.05$; Figure 7).

\subsubsection{Forearm Muscle Activity}

Forearm EMG activity for the first 10 to last 10 contractions did not differ for any pressure condition (all $\mathrm{P}>0.05$ ). 


\subsection{Discussion}

The purpose of this study was to evaluate changes in arterial blood flow and tissue perfusion before, during, and after rhythmic handgrip exercise with BFR across a range of occlusion pressures. The key findings were that: 1) at pressures below LOP the cardiovascular system was able to overcome the external pressure and increase blood flow to the working muscles during exercise, 2) relative reductions in blood flow at rest were similar to those during exercise, and 3) cuff pressures of 60 and $80 \%$ LOP reduced tissue saturation index and increased deoxyhemoglobin concentrations, which is consistent with an increase in metabolic stress and metabolite accumulation $[71,72]$, without completely occluding arterial blood flow. Taken together, these findings provide novel insight into hemodynamic changes during exercise with BFR and have important implications. Specifically, the relative level of blood flow occlusion measured at rest may approximate the degree of occlusion during exercise and application of moderate cuff pressure seems to offer an appropriate BFR occlusion stimulus for small muscle mass exercise.

\subsubsection{Assessment of Blood Flow during Exercise}

To the best of our knowledge, we are the first group to report alterations in blood flow before, during, and after exercise with BFR. Previous research has focused on examinations of blood flow at rest $[44,49,54,63]$ and immediately after exercise $[73$, 74] with BFR. However, measurement of blood flow during exercise is very difficult as the motion of the exercising limb may influence the artery being examined and consequently give rise to motion artifacts [73]. To circumvent this limitation, we 
implemented BFR during rhythmic handgrip exercise; an exercise modality that is often used to measure blood flow to exercising muscles and assess peripheral vascular health [75-77]. Our brachial artery blood flow data at rest and during exercise without occlusion ( $0 \%$ LOP) are consistent with previously reported data on blood flow during handgrip exercise $[78,79]$. Thus, we are confident that our assessment of blood flow during handgrip exercise yielded reliable results and that changes in blood flow with BFR were due to the occlusion pressure.

\subsubsection{Alterations in Blood Flow}

Prior to exercise, there were no significant differences in blood flow between the $60 \%$ and $80 \%$ and the $100 \%$ and $120 \%$ LOP conditions. These data are in agreement with that of Mouser and colleagues $[44,49]$ who demonstrated that under relative occlusion pressure, the reduction in blood flow does not linearly decrease with increasing pressure; as they reported similar blood flow values between $50-90 \%$ of LOP. This non-linear decrease in blood flow is unique to relative pressures as previous reports indicate a linear decrease in blood flow with absolute increases in cuff pressures above $80 \mathrm{mmHg}$ [54, 63]. These differences are likely due to individual differences in LOP and variations in absolute and relative cuff pressures. For example, an absolute pressure of $140 \mathrm{mmHg}$ would range from approximately $80-105 \%$ LOP for the participants in this study, which would completely occlude blood flow in the majority of participants (7 of 10). According to a recent questionnaire on the use of exercise with BFR, only $12 \%$ of practitioners are currently basing cuff pressure on LOP when implementing exercise with BFR [23]. 
These findings re-enforce the need to individualize pressures before performing exercise with BFR to ensure user safety and facilitate consistent training adaptations.

During exercise, blood flow increased in the $0 \%, 60 \%$, and $80 \%$ LOP conditions from rest. Thus, at pressures below LOP the cardiovascular system increased blood flow to the working muscles even despite the mechanical compression. However, blood flow was minimal $(<10 \mathrm{ml} / \mathrm{min})$ during the $100 \%$ and $120 \%$ LOP conditions. At these pressures, the cardiovascular system cannot overcome the external pressure. Moreover, while the absolute blood flow increased during exercise with the lower cuff pressures the relative reduction in blood flow due to the applied pressure at rest was, for the most part, maintained during exercise. Specifically, at $60 \%$ LOP blood flow was reduced by $\sim 20 \%$ at rest as well as during exercise. Likewise, at $80 \%$ LOP blood flow was reduced by $\sim 45 \%$ at rest and during exercise. This finding is important as these two pressures are often reported in the literature and shed light on previous speculations that cuff pressure would have to be increased during exercise to maintain the same level of blood flow restriction [54].

In the $0 \%$ LOP control condition blood flow remained elevated following exercise, however, this hyperemic response was blunted by the cuff across all cuff pressures. Post-exercise absolute blood flow decreased in the $60 \%$ and $80 \%$ LOP conditions from the exercise values. This is in contrast to the work by Downs and colleagues [73] as these authors speculated that post-exercise blood flow would be similar to blood flow during exercise due to the low loads used, or higher, due to postexercise reactive hyperemia. Although the $60 \%$ and $80 \%$ LOP conditions did reduce 
blood flow during exercise by $\sim 20 \%$ and $\sim 45 \%$ compared to the $0 \%$ control condition, the post-exercise reductions during these two conditions were much greater. Specifically, post- exercise blood flow was reduced by $\sim 50 \%$ and $\sim 70 \%$ during the $60 \%$ and $80 \%$ LOP conditions, respectively. These results may be explained by the skeletal muscle pump, which during exercise would increase venous return and allow for greater arterial blood flow. Following exercise, the absence of the skeletal muscle pump's assistance with venous return would augment venous pooling and venous pressure thus reducing the pressure gradient needed to achieve post-exercise hyperemia. If the goal of exercise with BFR is to maintain the relative reduction in blood flow, then cuff pressure should be held constant before and during exercise and reduced between sets. Alternatively, if the goal is to keep the reduction in absolute blood flow constant, then the cuff pressure set at rest should be increased during and after exercise, but to a greater extent during exercise. A long-term training intervention is required to elucidate the best strategy for restricting blood flow during exercise with BFR.

\subsubsection{Tissue Perfusion}

Results indicated that increased pressure reduced tissue saturation index. This relationship is similar to blood flow, which seems logical as blood flow and tissue perfusion are highly correlated $[80,81]$. There were, however, no differences in deoxyhemoglobin in the $80 \%, 100 \%$, and $120 \%$ LOP conditions. Although not measured in this study, this could be because pressures above $80 \%$ LOP completely occlude venous blood flow, and therefore there was no deoxyhemoglobin clearance from the muscle in these conditions. Ganesan and colleagues [71] proposed that BFR-induced hypertrophy is 
due to localized hypoxia and metabolite accumulation. With this in mind, our data suggest that cuff pressures above $60 \%$ LOP do cause tissue hypoxia and increase metabolite accumulation. Moreover, the finding that pressures greater than $80 \%$ LOP do not increase deoxyhemoglobin suggests that these higher pressures may not be entirely necessary. Higher pressures would likely increase discomfort and risk for adverse cardiovascular responses [82] without increasing metabolite accumulation. Based on the above results, cuff pressures between $60-80 \%$ LOP seem appropriate for increasing metabolic stress and metabolite accumulation without completely occluding blood flow and compromising individual safety.

In all pressure conditions above $0 \% \mathrm{LOP}$, tissue saturation index continued to decrease post-exercise. These results are consistent with previous reports on tissue saturation index following knee extension exercise with BFR [71, 73]. Suga and colleagues [30] reported that metabolic stress and metabolite accumulation induced from multiple-sets of BFR exercise is similar to that of high-intensity exercise, only if the cuff pressure is maintained during the rest period. If the goal of exercise with BFR is to create a hypoxic environment within the muscle and to increase the accumulation of metabolites to a similar level of high-intensity exercise, then the cuff pressure should be maintained between sets. Such a strategy would limit post-exercise hyperemia and consequently decrease tissue saturation index and increase metabolite build-up.

\subsubsection{Mean Arterial Pressure}

There were no differences in mean arterial pressure across different cuff pressures. This may be due to the small muscle mass used or the type of exercise used. It 
is possible that there would be a change in mean arterial pressure across pressures during dynamic exercise with larger muscle masses. Mean arterial pressure increased from before exercise to exercise in all conditions, and returned to pre-exercise levels within the post-exercise time period in the $0 \%$ and $60 \%$ LOP condition and nearly returned for the $80 \%$ LOP condition. However, in the $100 \%$ and $120 \%$ LOP conditions mean arterial pressure remained elevated during the post-exercise time period. These results are in agreement with Shoemaker and colleagues [83] who demonstrated that mean arterial pressure remained elevated with post-exercise circulatory occlusion following handgrip exercise. These differences can be explained by the muscle metaboreflex which is activated due to the accumulation of metabolites, and or the reduction in oxygen delivery [84], both of which were found to be elevated at higher pressures $(80 \%, 100 \%, 120 \%$ LOP). Thus, these pressures would have more fully activated this reflex, causing mean arterial pressure to remain elevated post-exercise [82]. It is important to note that this reflex is exaggerated in disease states in which perfusion to the muscle is impaired including hypertension, heart failure, and peripheral artery disease [82]. Therefore, caution should be taken before using BFR exercise in these populations, including using lower occlusion pressures (e.g., $<60 \%$ LOP), and normalizing the restrictive stimulus to the individual.

\subsubsection{Implications}

Exercise with BFR is gaining popularity among practitioners in clinical and applied sport training settings [23]. However, more care is needed to ensure that current practice matches the research to ensure the safety of this exercise modality [23]. With this in 
mind, our findings have implications for researchers, clinicians, coaches, and athletes. Specifically, researchers who use exercise with BFR may be able to better standardize their BFR stimulus before exercise without needing to measure blood flow during exercise, which is difficult. These results benefit clinicians by demonstrating that moderate cuff pressures $(\leq 80 \%$ LOP) do not occlude blood flow during small muscle mass exercise with BFR. Because deoxyhemoglobin concentration was the same at pressures above $80 \%$ LOP, higher cuff pressures may be unnecessary. Moreover, the use of higher cuff pressures could cause MAP to remain elevated during the rest period which would be contraindicated for populations that have an exacerbated blood pressure response to exercise. Finally, coaches and athletes including exercise with BFR in training programs may need to consider that individual LOP differs, which affects the stimulus for BFR induced improvements in muscle size and strength. It is recommended that practitioners base the cuff pressure on LOP if possible [17]. Accordingly, careful standardization of BFR stimulus during rest may facilitate more consistent training adaptations during exercise.

\subsubsection{Limitations}

We implemented BFR during rhythmic handgrip exercise, an exercise modality that is often used to measure blood flow to exercising muscles. This allowed us to measure blood flow during exercise with BFR for the first time. Handgrip exercise is, however, not without its limitations. Our findings relating to the hemodynamics of BFR with handgrip exercise are limited by the type of muscle contraction (quasi-isometric) and small muscle mass engaged. Thus, these findings may not be entirely transferrable to 
more dynamic exercises with BFR involving a larger muscle mass. More common exercises used in conjunction with BFR such as knee extension, bench press, and elbow flexion, would likely increase acute cardiovascular responses (i.e., heart rate, mean arterial pressure, cardiac output) to a much greater extent than that measured in this study. Thus, relative reductions in blood flow from various occlusion pressures are likely specific to the limb and exercise involved. Also, we did not measure vessel diameter during exercise, which may have impacted our blood flow data. However, if diameter did change during exercise, it is far more likely to increase rather than decrease; resulting in an underestimation of blood flow. Considering that the purpose of this exercise modality was to restrict, but not completely occlude arterial blood flow, this would result in conservative estimates for an overall effect. It is also important to note that we indirectly quantified metabolic stress by changes in tissue saturation index and deoxyhemoglobin concentration as opposed to a more direct assessment. Therefore, our interpretation of metabolic stress associated with BFR is limited to an indirect estimation.

We acknowledge that multiple sets are commonly used during exercise with BFR (e.g., 30 reps $\mathrm{x} 3$ sets) [23]. There was no change in forearm EMG activity for the first 10 to last 10 contractions suggesting that one set of handgrip exercise did not cause fatigue in the forearm muscles. These results are likely due to the light intensity (30\% MVC) utilized. Exercise with BFR involving larger muscle masses over multiple sets would likely increase EMG activity. Therefore, further investigation into the mechanisms of blood flow during exercise with BFR utilizing dynamic exercise, with multiple sets, in larger muscle groups is warranted. Lastly, we should note that these data were collected 
in a supine position, which is not the same position for most upper-body exercises that are performed seated or standing. This reiterates the need for future research exploring exercise with BFR during varying dynamic exercise.

\subsubsection{Summary}

During exercise with BFR, blood flow to the working muscles increased even despite the external pressure. Relative reductions in blood flow prior to exercise were generally maintained during exercise with BFR. Occlusion pressures ranging from 60$80 \%$ LOP provided considerable metabolic stress while still maintaining partial arterial blood flow, thus lowering relative risk of adverse cardiovascular responses to exercise with BFR, including prolonged increases in MAP during recovery. Overall, these findings provide novel insight into hemodynamic changes during exercise with BFR and serve as an important step for creating better BFR exercise guidelines.

\subsubsection{Acknowledgements}

Reprinted by permission from Springer: Nature European Journal of Applied Physiology. Limb blood flow and tissue perfusion during exercise with blood flow restriction. Kilgas, Matthew A., John McDaniel, Jon Stavres, Brandon S. Pollock, Tyler J. Singer, and Steven J. Elmer. Copyright (2018). 
Table 2: Participant demographic and anthropometric characteristics $(\mathrm{n}=10)$

\begin{tabular}{lc} 
Variable & Value \\
\hline Age (yr) & $27 \pm 4$ \\
Body mass (kg) & $82 \pm 14$ \\
Height (m) & $1.77 \pm 0.1$ \\
Body mass index (kg·m $\left.{ }^{-2}\right)$ & $26 \pm 3$ \\
Systolic blood pressure (mm Hg) & $118 \pm 5$ \\
Diastolic blood pressure (mm Hg) & $75 \pm 7$ \\
Triceps Skinfold (mm) & $10 \pm 3$ \\
Biceps Skinfold (mm) & $5 \pm 1$ \\
Upper arm circumference (cm) & $32 \pm 3$ \\
Upper arm muscle area (cm $\left.{ }^{2}\right)^{\mathrm{a}}$ & $59 \pm 13$ \\
\hline
\end{tabular}

Values are reported as Mean \pm SD

${ }^{a}$ estimated bone-free arm muscle area 


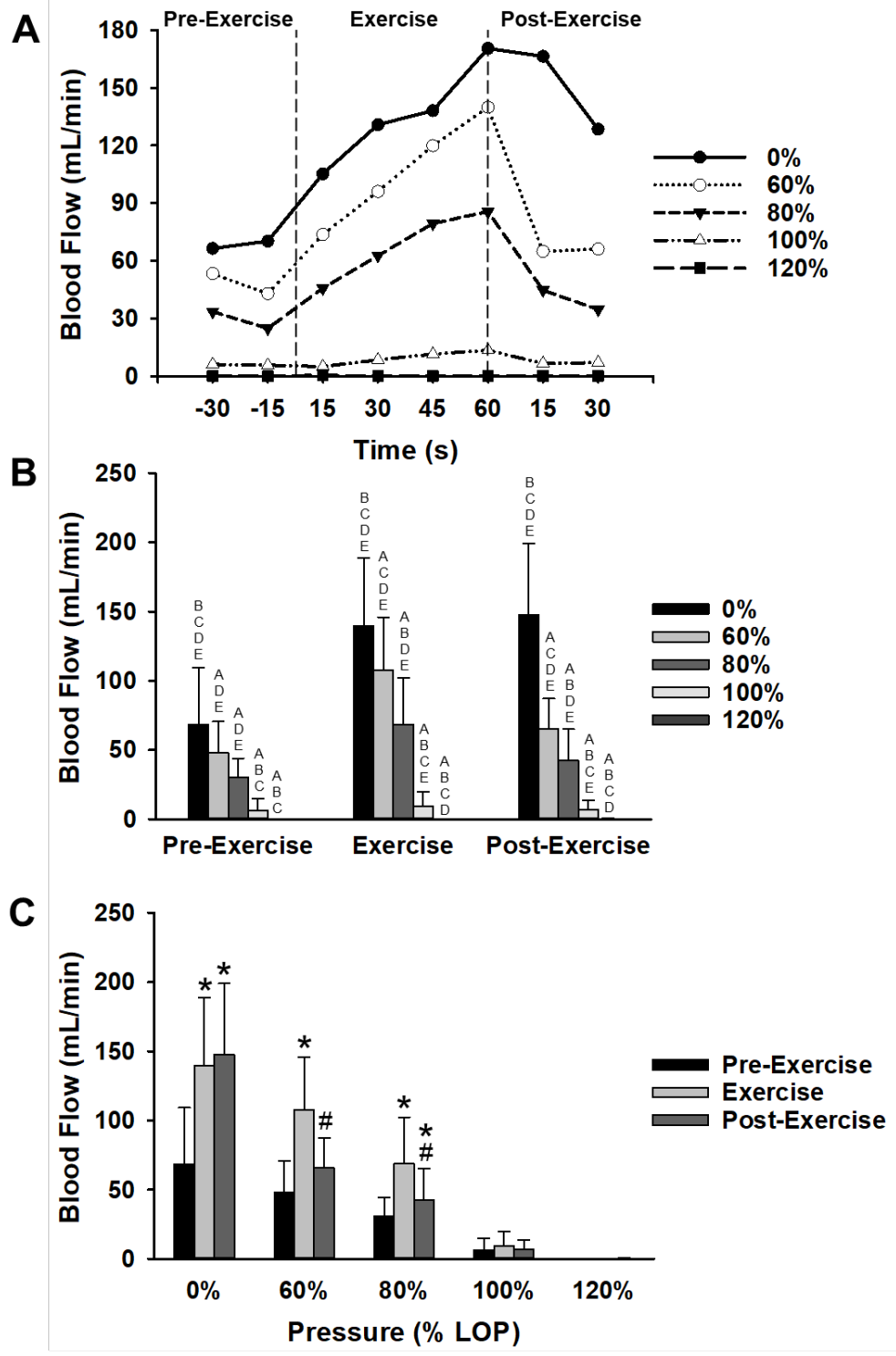

Figure 3: Time course of alterations in absolute brachial artery blood flow across different cuff pressures (A). Brachial artery blood flow measured during pre-exercise, exercise, and post-exercise with the cuff inflated (B). Blood flow different from $0 \%$, $60 \%, 80 \%, 100 \%$, and $120 \%(\mathrm{P}<0.05)$ are indicated by A, B, C, D, and E, respectively. Brachial artery blood flow measured at each cuff pressure for pre-exercise, exercise, and post exercise $(C)$. Blood flow different than pre-exercise and exercise $(p<0.05)$ are indicated by $*$ and \#, respectively. Data are as reported mean \pm SD. Note that, blood flow measured at $120 \%$ LOP was minimal. 


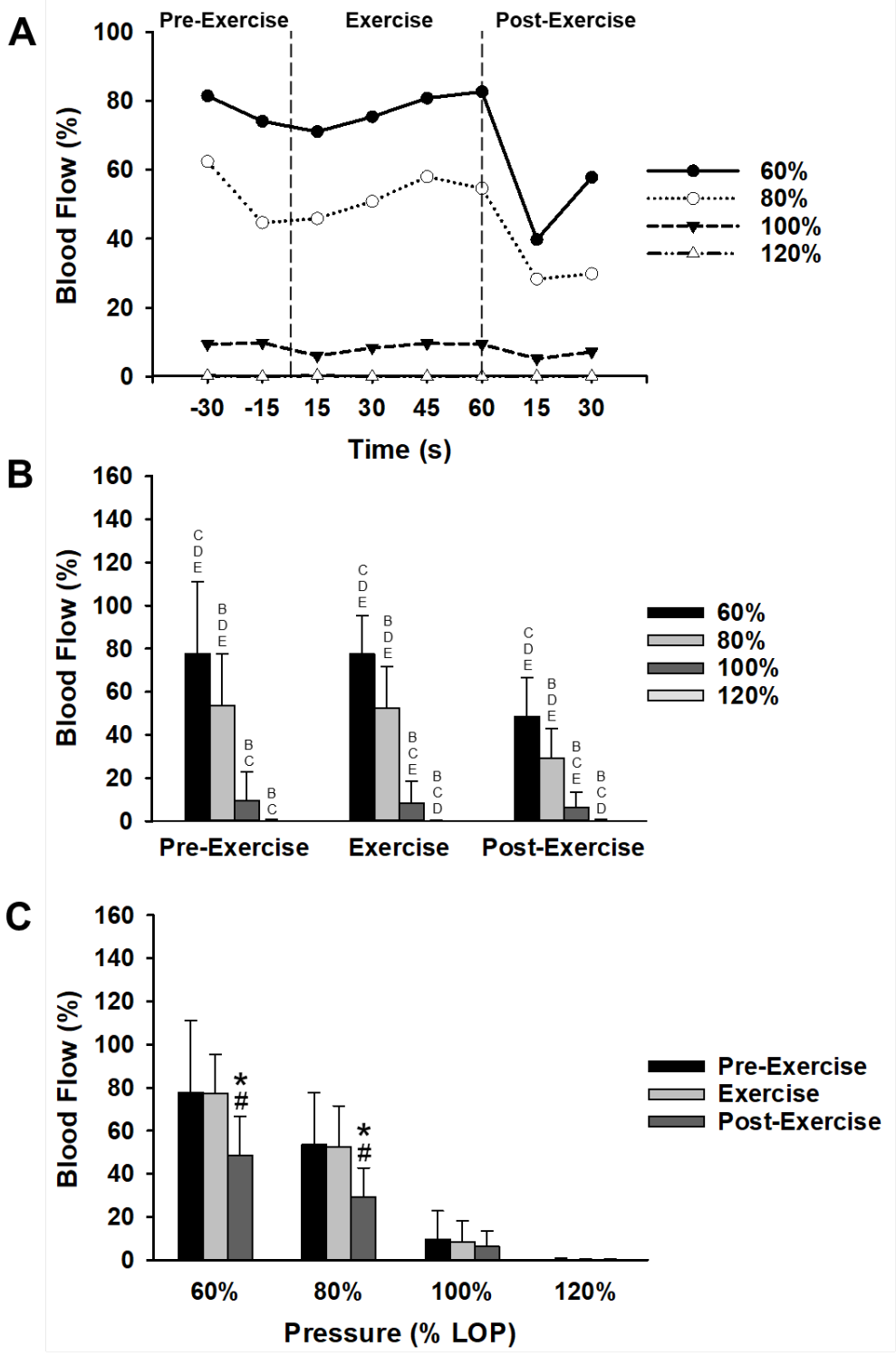

Figure 4: Time course of alterations in relative brachial artery blood flow across different cuff pressures (A). Brachial artery blood flow measured during pre-exercise, exercise, and post-exercise with the cuff inflated (B). Blood flow different from $0 \%$, $60 \%, 80 \%, 100 \%$, and $120 \%(\mathrm{P}<0.05)$ are indicated by $\mathrm{A}, \mathrm{B}, \mathrm{C}, \mathrm{D}$, and $\mathrm{E}$, respectively. Brachial artery blood flow measured at each cuff pressure for pre-exercise, exercise, and post exercise $(C)$. Blood flow different than pre-exercise and exercise $(\mathrm{p}<0.05)$ are indicated by * and \#, respectively. Blood flow different than exercise $(p<0.05)$. Data are reported as mean $\pm \mathrm{SD}$. Note that, blood flow measured at $120 \%$ LOP was minimal. 

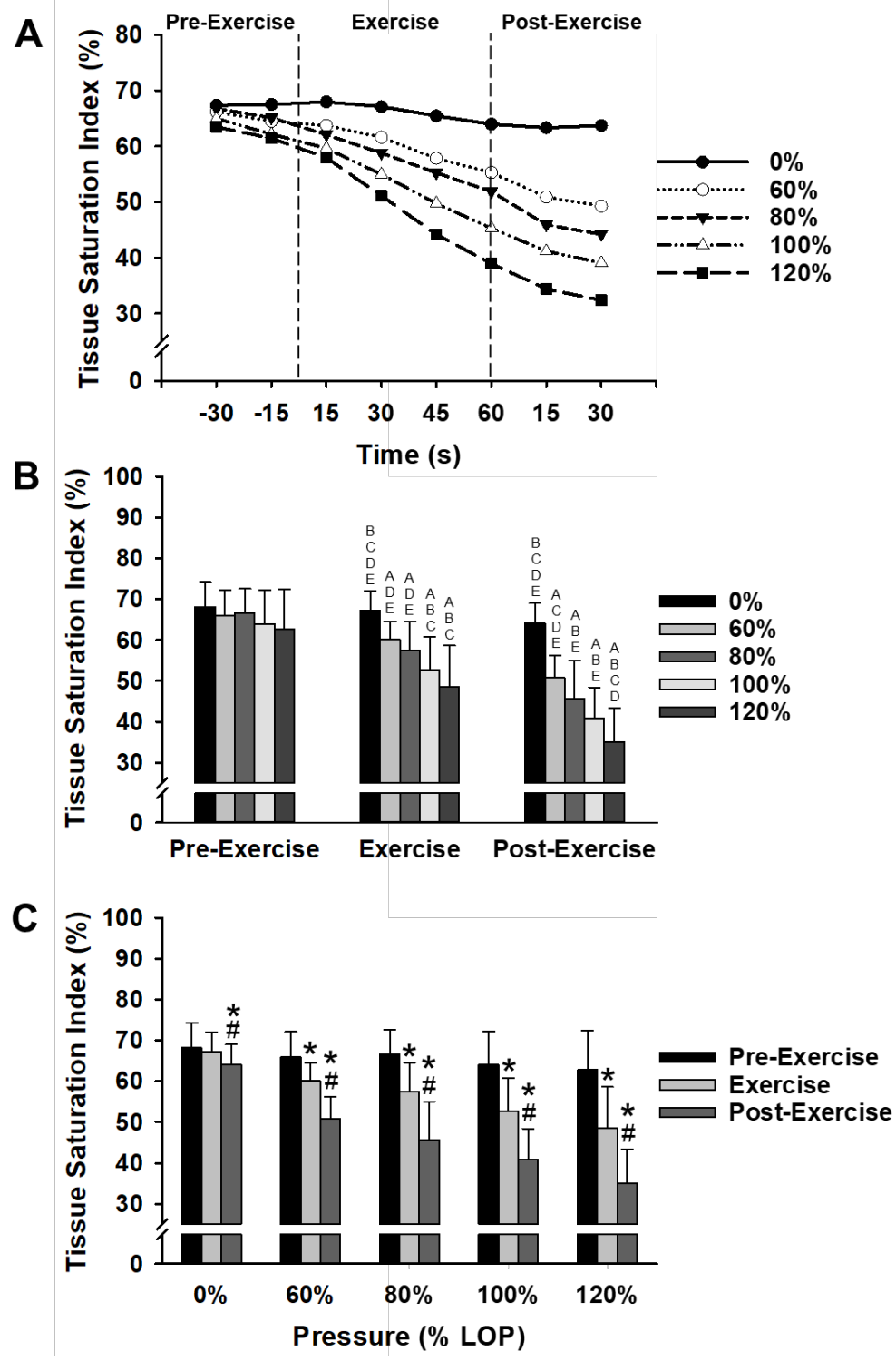

Figure 5: Time course of alterations in tissue saturation index (A) across different cuff pressures. Tissue saturation index measured during pre-exercise, exercise, and postexercise with the cuff inflated. (B) Tissue saturation index different from $0 \%, 60 \%, 80 \%$, $100 \%$, and $120 \%(\mathrm{P}<0.05)$ are indicated by A, B, C, D, and E, respectively. Tissue saturation index measured at each cuff pressure for pre-exercise, exercise, and post exercise $(C)$. Tissue saturation index different than pre-exercise and exercise $(p<0.05)$ are indicated by * and \#, respectively. Data are reported as mean $\pm \mathrm{SD}$. 

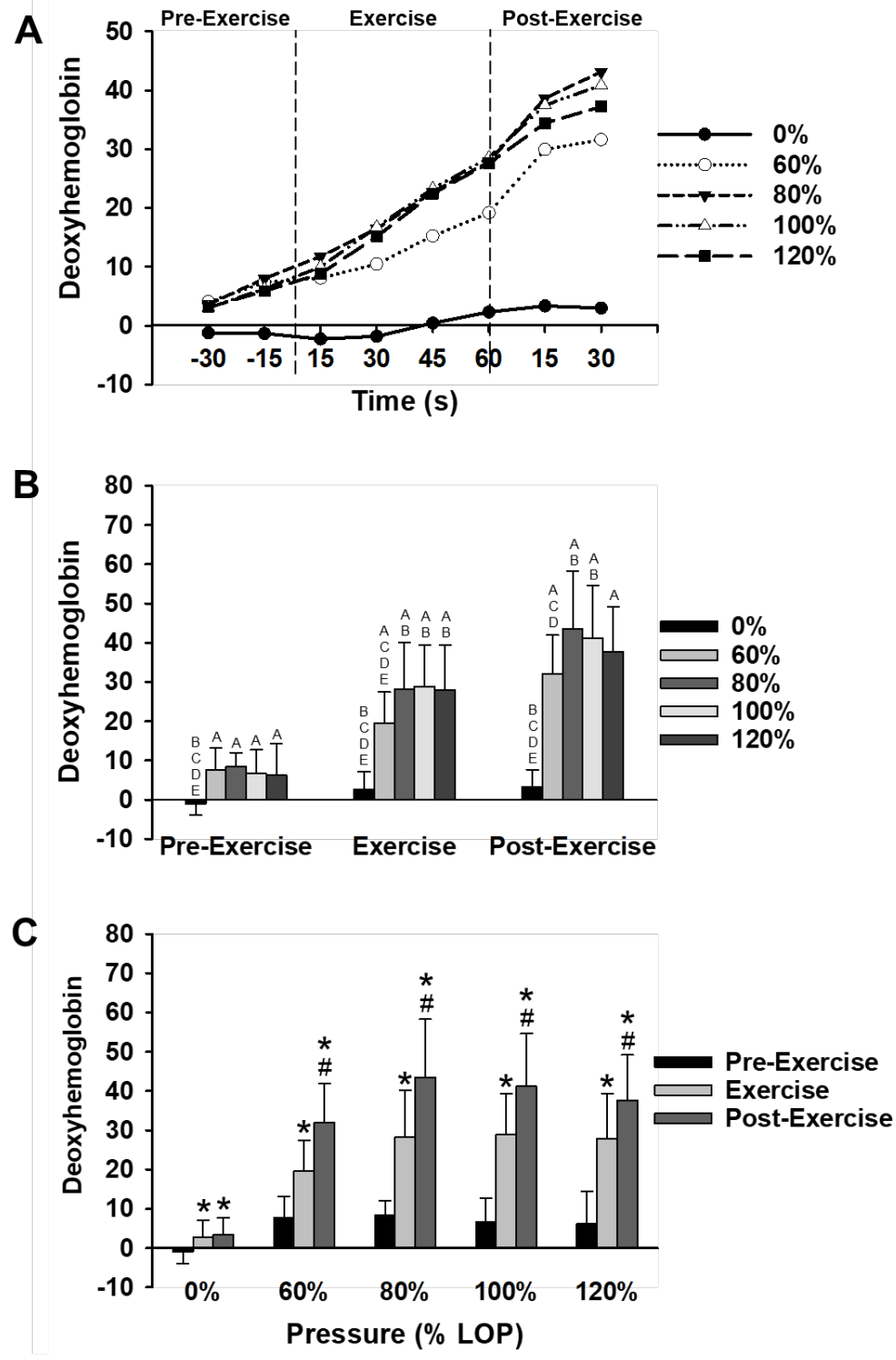

Figure 6: Time course of alterations in concentration of deoxyhemoglobin (A) across different cuff pressures. Concentration of deoxyhemoglobin measured during preexercise, exercise, and post-exercise with the cuff inflated (B). Concentration of deoxyhemoglobin different from $0 \%, 60 \%, 80 \%, 100 \%$, and $120 \%(\mathrm{P}<0.05)$ are indicated by A, B, C, D, and E, respectively. Concentration of deoxyhemoglobin measured at each cuff pressure for pre-exercise, exercise, and post exercise $(\mathrm{C})$. Concentration of deoxyhemoglobin different than pre-exercise and exercise $(\mathrm{p}<0.05)$ are indicated by * and \#, respectively. Data are reported as mean $\pm \mathrm{SD}$. 


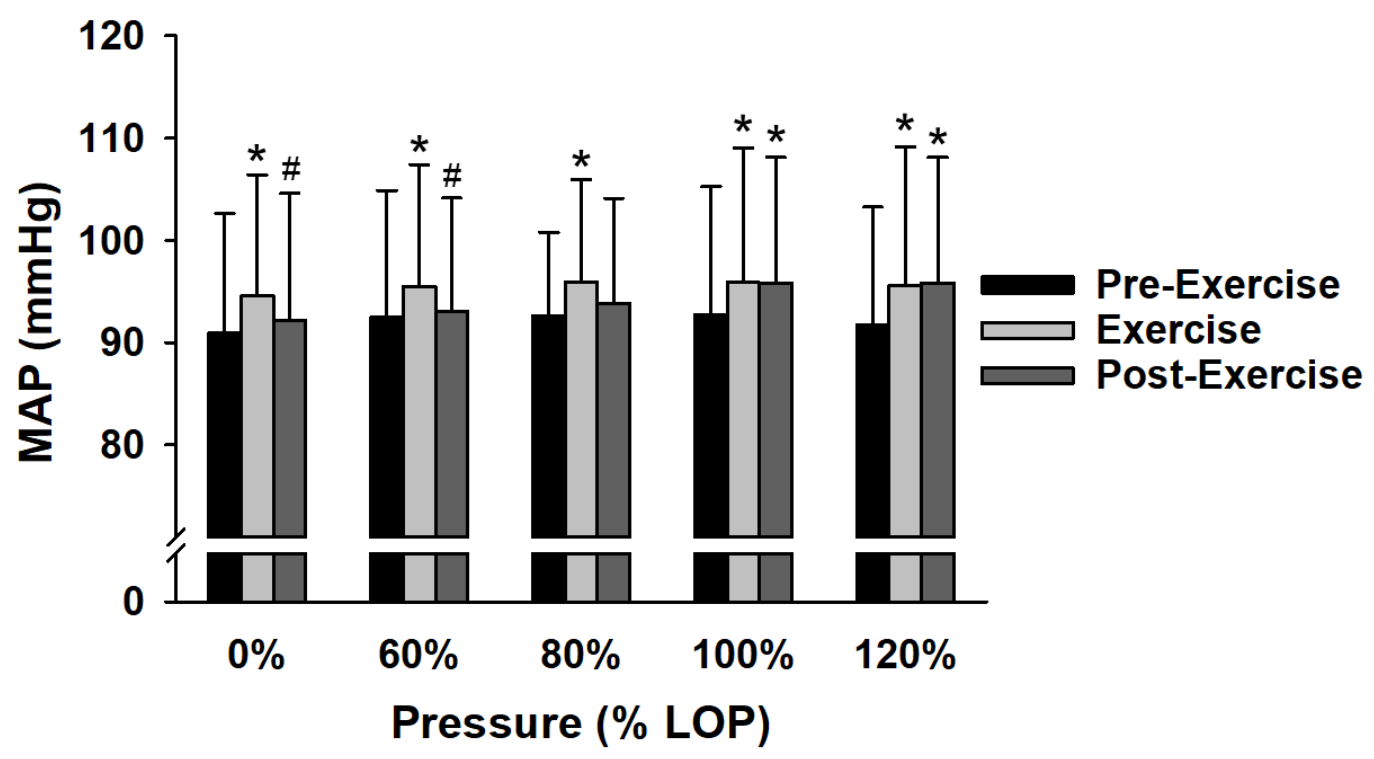

Figure 7: Alterations in mean arterial pressure (MAP) by time. Mean arterial pressure different than pre-exercise and exercise $(\mathrm{p}<0.05)$ are indicated by * and \#, respectively. Data are reported as mean $\pm \mathrm{SD}$. 


\section{Acute Cardiovascular, Metabolic, and Neuromuscular Effects of Cycling with Blood Flow Restriction}

\subsection{Introduction}

Exercise with blood flow restriction (BFR) is emerging as a safe and effective method for increasing muscle size and strength $[26,85]$. This exercise uses a pressurized cuff or tourniquet to partially restrict blood flow to and from the working muscles [17]. Blood flow restriction is usually combined with low-load resistance exercise training (e.g., 20-30\% of 1 repetition maximum) [23]. Similarly, low-intensity aerobic exercise training with BFR (e.g., cycling at $40 \%$ of $\mathrm{VO}_{2 \max }$ ) increases muscle size and strength as well as $\mathrm{VO}_{2 \max }$, time until exhaustion, and onset of blood lactate $[6,10,25,36-38,86]$. Thus, aerobic exercise with BFR may offer a unique 2-for-1 stimulus for improving both muscular and cardiovascular function. Recent evidence also suggests that aerobic exercise with BFR elicits a lower cardiovascular response (e.g., heart rate, cardiac output, mean arterial pressure) than resistance exercise with BFR [35]. With these factors in mind, several authors have proposed some novel applications for aerobic exercise with BFR. For example, this exercise mode could offer a more feasible option for individuals with cardiovascular risk factors [35] as well as an appropriate adjunct countermeasure for astronauts enduring long-duration space flight [87].

There are no standardized recommendations for the proper application of exercise with BFR. Theoretically, cuff pressure should be high enough to prevent the return of venous blood flow but low enough to maintain some arterial blood flow [88]. Further, 
cuff pressure should maximize metabolic stress [61, 62] (e.g., tissue desaturation, metabolite accumulation) without inducing excessive discomfort. Previous investigators [73] have assessed blood flow and tissue perfusion (oxygen availability, metabolite accumulation) before and after resistance exercise with BFR. Results indicated that postexercise blood flow was higher than pre-exercise blood flow when using lower cuff pressures, but not when using higher cuff pressures. Additionally, tissue perfusion decreased with BFR during exercise and did not recover following exercise when the cuff remained inflated. It is unclear if changes in hemodynamics during resistance exercise with BFR can be applied to aerobic exercise with BFR because there are differences in blood pressure, cardiac output, and the cyclical nature of the movement between the two exercise modes. To the best of our knowledge, no previous authors have reported changes in blood flow and tissue perfusion associated with aerobic exercise with BFR. Understanding how pressure affects blood flow and tissue perfusion is critical for maximizing the occlusion training stimulus and implementing aerobic exercise with BFR safely and effectively.

Reductions in blood flow during BFR exercise also contribute to the development of neuromuscular fatigue (i.e., reduction in maximal voluntary torque) [89]. Typically, greater neuromuscular fatigue is associated with increased growth hormone concentrations which may play a role in training adaptations $[21,90]$. While there is evidence of neuromuscular fatigue after resistance exercise with BFR [31, 91-93], previous reports documenting changes in neuromuscular function following aerobic exercise with BFR are mixed. Ogawa and colleagues [94] reported no change in isometric 
knee extensor torque following 30 min of walking with BFR. Conversely, Kim and colleagues [8] reported significant reductions in isometric knee extensor torque following cycling with BFR until failure. However, according to the authors, these reductions were not outside the measurement error. Nevertheless, any potential reductions in knee extensor torque could be due to factors that reside in the brain and spinal column (central fatigue), the muscles themselves (peripheral fatigue), and/or some combination of the two (central and peripheral fatigue). Given these varied results $[8,94]$ and the task-specific nature of fatigue [95], additional research is needed to confirm how aerobic exercise with BFR influences both the development and recovery of neuromuscular fatigue.

The purpose of this study was to compare changes in blood flow, tissue perfusion, and neuromuscular function to acute cycling exercise with and without BFR. Specifically, comparisons were made between: 1) low-load cycling $\left.\left(40 \% \mathrm{VO}_{2 \max }\right), 2\right)$ low-load cycling with moderate BFR, 3) low-load cycling with high BFR, and 4) highload cycling $\left(80 \% \mathrm{VO}_{2 \max }\right)$. We hypothesized that low-load cycling with BFR would decrease blood flow and tissue saturation index and increase concentrations of deoxyhemoglobin. These responses would be further exacerbated by increased cuff pressure. We also hypothesized that cycling with BFR would impair end exercise maximal knee extensor isometric torque. Based on previous reports [31, 91] we hypothesized that these impairments in neuromuscular function would be mainly due to peripheral mechanisms. 


\subsection{Methods}

\subsubsection{Participants}

Ten active men between 18-44 yrs volunteered to participate in this study (Table 1). All participants self-reported that they performed aerobic exercise at moderate to high-intensity for at least $150 \mathrm{~min} /$ week, which is consistent with ACSM guidelines [96]. Body composition and lower limb lean mass were assessed using dual energy $\mathrm{x}$-ray absorptiometry (Discovery Wi, Hologic Inc, Marlborough, MA, USA). Participants were excluded from the study if they used nicotine products, had diabetes, or had any cardiopulmonary disorders. Following the initial screening, participants were informed of the purpose of the study, the risks involved, and gave informed written consent. This study was approved by the Institutional Review Board at Michigan Technological University.

\subsubsection{Study Overview}

In this investigation, we used a single group repeated measures design. Participants reported to the laboratory on 5 separate days separated by at least $48 \mathrm{hrs}$. Subjects were told not to perform at vigorous physical activity, or use any drugs, alcohol, or tobacco for 12 hours prior to each session. All laboratory visits performed at approximately the same time of day, in a thermoneutral environment $\left(25^{\circ} \mathrm{C}\right)$. During the initial laboratory visit, participants were familiarized with the measurement of neuromuscular function, performed a submaximal cycling protocol, and completed a graded exercise test for determination of maximum oxygen consumption $\left(\mathrm{VO}_{2 \max }\right)$. For the remaining experimental laboratory visits, participants completed one of the four 
cycling conditions which were presented in a randomized fashion. The conditions consisted of: 1) low-load cycling at $\left.40 \% \mathrm{VO}_{2 \max }(\mathrm{LL}), 2\right)$ low-load cycling at $40 \%$ $\mathrm{VO}_{2 \max }$ with BFR set at $60 \%$ limb occlusion pressure (BFR60), 3) low-load cycling at $40 \% \mathrm{VO}_{2 \max }$ with BFR set at $80 \%$ occlusion pressure (BFR80), and 4) high-load cycling at $80 \% \mathrm{VO}_{2 \max }(\mathrm{HL})$. Prior to each cycling trial, baseline neuromuscular function was assessed and limb occlusion pressure was identified. Participants then completed an intermittent cycling protocol (6 sets of 2 min cycling intervals with 1 min recovery between sets). Tissue perfusion and gas exchange data were recorded throughout the cycling trial. Blood flow was measured during the recovery periods. Immediately after the cycling trial, neuromuscular function was assessed again (Figure 8).

\subsubsection{Oxygen consumption and Peak Aerobic Power}

To establish a linear relationship between steady-state $\mathrm{VO}_{2}$ and power output, participants completed a submaximal cycling protocol on an electromagnetically braked cycle ergometer (Velotron Elite; RacerMate Inc., Seattle, WA, USA). Specifically, participants cycled at 40,80, 120, and $160 \mathrm{~W}$ for 4 min using a self-selected pedaling rate. Gas exchange data were measured continuously using open-circuit spirometry (True Max 2400; Parvo Medics, Sandy, UT, USA). The metabolic measurement system was calibrated with a $3 \mathrm{~L}$ calibration syringe and medical gases of known concentrations $\left(16.00 \% \mathrm{O}_{2}, 4.00 \% \mathrm{CO}_{2}\right.$, balanced $\left.\mathrm{N}_{2}\right)$. Heart rate was measured continuously using a Polar transmitter (Polar Electro OY, Kempele, Finland). Gas exchange and heart data were averaged every $15 \mathrm{~s}$ throughout the test. After a 15 min break, participants then performed a graded exercise test until task failure as described by Lucia and colleagues 
[97]. The protocol began at $40 \mathrm{~W}$ and increased $5 \mathrm{~W}$ every $12 \mathrm{~s}$. The test was terminated voluntarily by the participant, or when pedaling rate could no longer be maintained at 70 rpm, despite verbal encouragement. The highest $30 \mathrm{~s}$ average of $\mathrm{VO}_{2 \max }$ and heart rate achieved during the test were recorded. Power outputs that elicited $40 \%$ and $80 \%$ of $\mathrm{VO}_{2 \max }$ were estimated by interpolating the linear relationship between $\mathrm{VO}_{2}$ and power output.

\subsubsection{Cycling Exercise}

Prior to the intermittent cycling protocol, participants rested on the cycle ergometer for $5 \mathrm{~min}$ for collection of baseline responses. Following this baseline period, the cuff was inflated to 60 or $80 \%$ limb occlusion (BFR conditions only) while participants rested for $1 \mathrm{~min}$. Note that, for the LL and HL cycling conditions 1 min of rest was also

provided. Subsequently, participants completed 6 sets of 2 min of cycling intervals with 1 min between sets. An intermittent cycling protocol was selected because previous research suggests that work-rest periods while keeping the pressure cuff inflated has benefits over continuous cycling with BFR [34]. For the BFR cycling conditions, blood flow was restricted in each leg using a $10 \mathrm{~cm}$ wide nylon pneumatic cuff (Hokanson, Belleview, WA, USA) wrapped around the thigh at the most proximal location. The cuff pressure was set and maintained using a rapid cuff inflator (Hokanson, Belleview, WA, USA). The pressure in the cuff was sustained throughout the entire cycling protocol and deflated immediately after the participant completed the last set of cycling. 


\subsubsection{Blood Flow}

Blood flow velocity $\left(\mathrm{V}_{\text {mean }}\right)$ and vessel diameter $\left(\mathrm{V}_{\mathrm{d}}\right)$ were measured with a Logiq e ultrasound system (General Electric Medical Systems, Milwaukee, WI, USA) equipped with a linear array transducer operating at an imaging frequency of $12 \mathrm{MHz}$ and Doppler frequency of $5 \mathrm{MHz}$. Doppler pulse wave spectrum and ultrasound images were continuously recorded throughout each time period. Vessel diameters were determined by averaging the perpendicular distance between the superficial and deep walls of the superficial femoral artery at three nonconsecutive $\mathrm{R}$ waves during the last $15 \mathrm{~s}$ of each recording. Measurements of $\mathrm{V}_{\text {mean }}$ were obtained with the probe positioned to maintain an insonation angle of $\leq 60^{\circ}$. Mean blood velocity was averaged across $15 \mathrm{~s}$ intervals throughout the recording. Importantly, blood velocity data obtained with Doppler ultrasound are reliable [66] which is notable given the complex nature of blood velocity during dynamic muscle contractions. Using arterial diameter and mean blood velocity, blood flow was calculated as Blood Flow $=\mathrm{V}_{\text {mean }} * \pi^{*}\left(\mathrm{~V}_{\mathrm{d}} / 2\right)^{2 *} 60$ in accordance with the methods described by Wray and colleagues [98]. Blood flow was averaged throughout the 1 min recovery interval.

Limb occlusion pressure was determined while the participant was seated on the cycle ergometer. Their right foot was positioned on a stool next to the ergometer. Their hip was abducted slightly and their knee angle was $\sim 90^{\circ}$. The pressure cuff was wrapped around the right thigh, at the same position as exercise. The ultrasound probe was positioned distal to the cuff over the superficial femoral artery. Limb occlusion pressure was identified by inflating the cuff to $75 \mathrm{mmHg}$, and slowly increasing the pressure until 
blood flow velocity reached zero based on the absence of the Doppler spectrum. The minimum pressure required to do this was recorded as the limb occlusion pressure. The measurement of limb occlusion pressure using this method in our laboratory was reliable across exercise sessions (ICC $=0.89 ; 95 \%$ CI $0.80-0.94)$.

\subsubsection{Tissue Perfusion}

A continuous-wave near-infrared spectroscopy device (PortaLite; Artinis Medical Systems BV, Elst, The Netherlands) was utilized to detect changes in the concentrations of oxygenated hemoglobin and deoxygenated hemoglobin. Wavelengths (760 and 850 $\mathrm{nm}$ ) were emitted from LEDs with an inter-optode distance of $3.5 \mathrm{~cm}$. A differential pathlength factor of 4.0 was used to correct for photon scattering within the tissue. Data were collected at $10 \mathrm{~Hz}$ (Oxysoft; ArtinisMedical Systems BV, Elst, The Netherlands). The sensor was placed midway between the anterior superior iliac spine and the proximal patella parallel to the muscle fibers. The sensor was attached with double-sided tape and wrapped in an opaque bandage to prevent ambient light from reaching the sensor. Tissue saturation index (tissue saturation index $=$ oxyhemoglobin $/$ [deoxyhemoglobin + oxyhemoglobin]) was calculated using integrated software (Oxysoft; ArtinisMedical Systems BV, Elst, The Netherlands). The average tissue saturation index over the last 10 $\mathrm{s}$ of each time period was recorded. Changes in deoxyhemoglobin were assessed by calculating the difference between the average value of the last $10 \mathrm{~s}$ of each time period and the last $10 \mathrm{~s}$ of data recorded prior to inflating the cuff. This near-infrared spectroscopy system is reliable for measurement of tissue saturation index during leg exercise [99]. 


\subsubsection{Metabolic, Cardiorespiratory, and Perceptual Responses}

Oxygen consumption, ventilation, and heart rate were recorded using the metabolic measurement system described above. These data were averaged over the last $30 \mathrm{~s}$ of the exercise and recovery time periods. Perceptual responses (rating of perceived exertion and pain) were recorded during the last $30 \mathrm{~s}$ of each exercise and recovery period. Whole body rating of perceived exertion was assessed using a Borg 6-20 scale [100]. Pain was assessed using an 11-point numeric rating scale [101]. A blood sample was collected from the fingertip $(5 \mu l)$ during baseline and $1 \mathrm{~min}$ after the final exercise interval from which blood lactate concentration was determined (Lactate Plus; Nova Biomedical, Waltham, MA, USA).

\subsubsection{Neuromuscular Function}

Participants were positioned on an isokinetic dynamometer (Biodex 4, Biodex Medical Systems, NY, USA), at a hip angle of $85^{\circ}$, and a knee angle of $90^{\circ}$. A seat belt and ankle strap were used to minimize hip and ankle movement. To measure knee extension maximal voluntary isometric contraction (MVIC) torque, participants were instructed to "push as hard and as fast as possible" against an immoveable pad.

Standardized verbal encouragement was provided to the participant. Evoked torque was elicited by transcutaneous electrical stimulation over the knee extensors using a computer-controlled stimulator (D185; Digitimer, Welwyn Garden City, UK). The stimulating cathode was placed over the quadriceps femoris $10 \mathrm{~cm}$ distal to the anterior superior iliac spine and the anode was placed $2 \mathrm{~cm}$ proximal to the proximal border of the patella [102]. An electrical pulse (singlet, square wave, 100- $\mu$ s duration) was used to 
elicit a superimposed twitch at the peak torque level during the MVIC, and an additional potentiated resting twitch was triggered upon relaxation $(\sim 2 \mathrm{~s})$ following the MVIC. Stimulation intensity was determined based off no increase in twitch force despite increasing the stimulation current. A further increase of $20 \%$ was added to ensure that the stimulation was supramaximal. Voluntary activation was assessed using the interpolated twitch technique, and calculated as voluntary activation $=100 \times\left(1-\mathrm{T}_{\text {interpolated }} / \mathrm{T}_{\text {control }}\right)$, where $T_{\text {interpolated }}$ was the size of the interpolated twitch and $T_{\text {control }}$ was the amplitude of the control twitch produced by stimulation of the muscle in a relaxed but potentiated state [103]. Rate of torque development, time to peak torque, as well as the time to halfrelaxation were calculated using a customized routine (Spike 2; Cambridge Electronics Design, Cambridge, UK). Rate of torque development at the start of the MVIC was calculated as the peak tangential torque using a moving mean method of the torque-time curve over the first $400 \mathrm{~ms}$ from the onset of contraction [104]. Time to peak torque was defined as the slope of the force-time curve from baseline to peak $\mathrm{T}_{\text {control }}$ torque. Half relaxation time is defined as the slope of the line from peak $\mathrm{T}_{\text {control }}$ torque to half its value. These measurements were obtained at baseline, and at 1, 5, and $10 \mathrm{~min}$ after the exercise. The measurement of MVIC using this equipment in our laboratory was reliable across exercise sessions $(\mathrm{ICC}=0.91 ; 95 \%$ CI 0.76-0.98).

\subsubsection{Statistical Analysis}

Separate two-way repeated measures analysis of variance (ANOVA) procedures were used to evaluate the effect of cycling condition and time (baseline, cuff inflate, recovery interval number) on changes in blood flow. If a significant main effect of cycling 
condition was found, then subsequent post-hoc tests (Fisher's least significant difference) were used to determine where the differences occurred. If a significant effect of time was found, blood flow values over the recovery intervals were pooled together and additional paired samples t-tests were performed to analyze simple main effects.

Separate two-way repeated measures ANOVA procedures were used to evaluate the effect of cycling condition and time (baseline, cuff inflate, exercise interval number, recovery interval number) on changes in tissue saturation index, deoxyhemoglobin, $\mathrm{VO}_{2}$, heart rate, ventilation, RPE, and pain. If a significant main effect of cycling condition was found, then subsequent post-hoc tests (Fisher's least significant difference) were used to determine where the differences occurred. Additionally, if a significant main effect of time or condition was found for tissue saturation index or deoxyhemoglobin, data for exercise intervals and also recovery intervals were pooled and additional paired samples t-tests were used to evaluate simple main effects.

A repeated measures ANOVA was used to evaluate the interaction of cycling condition and time (baseline to post-exercise) on whole blood lactate. If a significant interaction was identified then a series of $2 \times 2$ repeated measures ANOVAs comparing each cycling condition at baseline and post-exercise were used to determine where the interactions occurred.

Finally, two-way repeated measures ANOVAs were used to evaluate the effect of cycling condition and time (baseline, post-1 min, post-5 min, post-10 min), on changes in MVIC, $\mathrm{T}_{\text {control, }}$ voluntary activation, rate of torque development, time to peak torque, and 
half relaxation time. If a significant main effect of cycling condition was found, then subsequent post-hoc tests (Fisher's least significant difference) were used to determine where the differences occurred. If a significant main effect of time was found paired samples t-tests were used to evaluate simple effects of time. If a significant interaction of cycling condition and time was found, a series of $2 \times 2$ repeated measures ANOVAs comparing each cycling condition at baseline and 1 min post-exercise were used to determine where the interactions occurred. Partial eta squared $\left(\eta p^{2}\right)$ was calculated as a measure of effect sizes with $\eta p^{2} \geq 0.01$ indicating small, $\geq 0.059$ medium, and $\geq 0.138$ large effects, respectively [105]. Statistical procedures were performed using SPSS 22 (Armonk, NY, USA). Data are reported as mean $\pm \mathrm{SD}$ and alpha was set to 0.05 .

\subsection{Results}

\subsubsection{Cycling Trials}

Cuff pressures for the BFR 60 and BFR 80 cycling conditions were $125 \pm 12$ and $164 \pm 15 \mathrm{~mm} \mathrm{Hg}$, respectively. Mean power outputs were $89 \pm 18 \mathrm{~W}$ for the LL, BFR 60, BFR 80 cycling conditions, and $240 \pm 36 \mathrm{~W}$ for the HL cycling condition. Two participants were unable to complete the last cycling interval in the BFR 80 condition due to lightheadedness and/or extreme pain at the site of the cuff. The cuff pressure was therefore released, and these side-effects were greatly reduced. These participants still completed the post-exercise assessment of neuromuscular function $1 \mathrm{~min}$ following the release of the occlusion cuff. For these two participants, tissue perfusion, metabolic, 
cardiorespiratory, and perceptual data for the last cycling interval were excluded from the analysis.

\subsubsection{Blood Flow}

The repeated measures ANOVA revealed significant main effects of cycling condition $\left(\mathrm{P}<0.01, \eta \mathrm{p}^{2}=0.887\right)$ and time $\left(\mathrm{P}<0.01, \eta \mathrm{p}^{2}=0.933\right)$ and cycling condition $\mathrm{x}$ time interaction $\left(\mathrm{P}<0.01, \eta \mathrm{p}^{2}=0.802\right)$ on blood flow. In general blood flow was reduced in the BFR cycling conditions compared to the non-BFR conditions (Figure 9A) and tended to decrease further in the BFR 80 condition $(\mathrm{P}=0.07)$ compared to BFR 60 . Within in each time period, blood flow did not differ between conditions at baseline, decreased with cuff inflation for BFR 60 and BFR 80 (both $\mathrm{P}<0.05$ ), and differed between all conditions during the recovery periods $(\mathrm{HL}>\mathrm{LL}>\mathrm{BFR} 60>\mathrm{BFR}$ 80; all $\mathrm{P}$ $<0.05$, Figure 9B).

\subsubsection{Tissue Perfusion}

Results from the repeated measures ANOVA procedures for tissue saturation index and deoxyhemoglobin revealed significant main effects of cycling condition $(\mathrm{P}<0.01$, $\eta p^{2}=0.741 ; \mathrm{P}<0.01, \eta p^{2}=0.783$, respectively $)$ and time $\left(\mathrm{P}<0.01, \eta \mathrm{p}^{2}=0.599 ; \mathrm{P}<\right.$ $0.01, \eta \mathrm{p}^{2}=0.687$, respectively) as well as a cycling condition $\mathrm{x}$ time interaction $(\mathrm{P}<$ $0.01, \eta p^{2}=0.511 ; \mathrm{P}<0.01, \eta p^{2}=0.683$, respectively). Overall, tissue saturation index was higher in LL, and HL, than it was in the BFR conditions (all $\mathrm{P}<0.05$, Figure 10A). Tissue saturation index was reduce in the BFR 80 condition compared to the BFR $60(\mathrm{P}<$ 0.01). Changes in tissue saturation index within each time period are illustrated in Figure 
10C. Compared to exercise, tissue saturation index increased during recovery for HL and LL cycling conditions (both $\mathrm{P}<0.05$ ). There was no difference in tissue saturation index during exercise and recovery within the BFR 60 and BFR 80 conditions. In general, concentrations of deoxyhemoglobin were higher in the BFR conditions (all $\mathrm{P}<0.05)$ and increased with pressure $(\mathrm{P}<0.05)$. There was a trend for concentrations of deoxyhemoglobin to be lower in LL than HL $(\mathrm{P}=0.06)$. Changes in concentrations of deoxyhemoglobin with in time period are illustrated in Figure 10D. Deoxyhemoglobin did not differ between exercise and recovery periods in the HL and LL conditions (both P $>0.05$ ) but increased during the recovery period for the BFR 60 and BFR 80 conditions (both $\mathrm{P}<0.01$ ).

\subsubsection{Metabolic, Cardiovascular, and Perceptual Responses}

The repeated measures ANOVA procedures revealed main effects of cycling condition (all $\mathrm{P}<0.01$, all $\eta \mathrm{p}^{2}>0.678$ ) and time (all $\mathrm{P}<0.01$, all $\eta \mathrm{p}^{2}>0.818$ ) as well as a cycling condition $\mathrm{x}$ time interaction (all $\mathrm{P}<0.01$, all $\eta p^{2}>0.511$ ) for all variables $\left(\mathrm{VO}_{2}\right.$, heart rate, ventilation, RPE, pain, lactate). Due to the intermittent nature of the cycling protocol, $\mathrm{VO}_{2}$ displayed a general "sawtooth" pattern for all conditions and overall was highest for HL (all P > 0.05, Figure 11). Heart rate was elevated in the BFR conditions compared to LL (both $\mathrm{P}<0.05$ ) but heart rate in the BFR 80 condition did not differ from HL $(\mathrm{P}=0.30)$. Ventilation was highest in the HL condition followed by BFR 80 , BFR 60, and LL respectively (all $\mathrm{P}<0.05$ ). Cycling with BFR caused an increase in RPE compared to LL (both $\mathrm{P}<0.05$ ), but RPE in the BFR 80 condition was not different from HL $(P=0.30)$. Pain was generally low during LL and HL but increased with BFR and 
increased pressure (all $\mathrm{P}<0.05$ ). Most notably, pain during BFR80 increased steadily and approached maximum values at the end of the final interval. Compared to baseline, end exercise whole blood lactate increased in the HL, BFR 60, and BFR 80 conditions (all $\mathrm{P}<0.05$ ). The increase in blood lactate for $\mathrm{HL}$ and BFR80 was greater than that for BFR60 but did not differ between HL and BFR 80 conditions $(\mathrm{P}=0.86)$.

\subsubsection{Neuromuscular Function}

The repeated measure ANOVA procedures indicated significant main effects of cycling condition for MVIC torque, $\mathrm{T}_{\text {control, }}$, rate of torque development, and time to peak torque (all $\mathrm{P}<0.01$, all $\eta \mathrm{p}^{2}>0.348$; Figure 12). Significant main effects of time were found for MVIC, voluntary activation, $\mathrm{T}_{\text {control, }}$, Time to peak torque, and half relaxation time (all $\mathrm{P}<0.01$, all $\eta \mathrm{p}^{2}>0.525$ ). Significant cycling condition $\mathrm{x}$ time interactions were found for MVIC, voluntary activation, $\mathrm{T}_{\text {control, }}$ time to peak torque, and rate of torque development $\left(\mathrm{P}<0.01\right.$, all $\left.\eta \mathrm{p}^{2}>0.258\right)$. In general, MVIC torque produced in the BFR conditions was lower than the non-BFR conditions and was further reduced with increased pressure. Reductions in MVIC torque from baseline to 1 min post-exercise for the BFR 60 condition were greater than those for LL and HL (both $\mathrm{P}<0.05$ ) but less than those for BFR $80(\mathrm{P}=0.02)$. Compared to baseline, MVIC torque was reduced at $10 \mathrm{~min}$ post-exercise for all conditions and was lowest for the BFR80 condition (all $\mathrm{P}<0.05$ ). Reductions in voluntary activation from baseline to 1 min post-exercise were greater in BFR 80 compared to all other conditions (all $\mathrm{P}<0.05$ ). Voluntary activation did not differ from baseline at $10 \mathrm{~min}$ post in any condition (all $\mathrm{P}>0.05$ ). For $\mathrm{T}_{\text {control }}$, BFR 80

was lower than LL and BFR 60 (both $\mathrm{P}<0.05)$, but not different than HL $(\mathrm{P}=0.10)$. The 
baseline to 1 min post-exercise reduction in $\mathrm{T}_{\text {control }}$ was greater in $\mathrm{BFR} 80$ than all other conditions (all $\mathrm{P}<0.05$ ). The $\mathrm{T}_{\text {control }}$ for all of the conditions were reduced 10 min postexercise compared to baseline (all $\mathrm{P}<0.05$ ). BFR 80 was generally lower than all other conditions for rate of torque development, and time to peak torque (all $\mathrm{P}<0.05$ ).

Similarly, the baseline to $1 \mathrm{~min}$ change in rate of torque development and time to peak torque for BFR 80 was greater than all other conditions (all $\mathrm{P}<0.05$ ). The rate of torque development for both BFR conditions was reduced 10 min post-exercise (both $\mathrm{P}<0.05$ ). Compared to baseline, time to peak torque was reduced for all conditions 10 min postexercise (all $\mathrm{P}<0.05)$. Finally, half relaxation time was not different than baseline 10 min post-exercise in the LL condition $(\mathrm{P}=0.13)$ but was increased in all other conditions (all $\mathrm{P}<0.05$ ).

\subsection{Discussion}

In this investigation, we integrated measurements of blood flow, tissue perfusion, and neuromuscular function to better characterize the effects of BFR during aerobic exercise. Our main findings were that cycling with BFR: 1) caused a reduction in blood flow and tissue perfusion and augmented metabolite accumulation compared to LL cycling, 2) lowered cardiorespiratory responses compared to high-load cycling, and 3) compromised knee extensor torque through a combination of peripheral and central mechanisms. Collectively, these results indicate that low load cycling exercise with BFR at $60 \%$ limb occlusion provided considerable metabolic stress (i.e., decreased tissue perfusion) with moderate discomfort while resulting in lower cardiorespiratory strain and perceived effort than high-load cycling. 


\subsubsection{Blood Flow and Tissue Perfusion}

To the best of our knowledge, we are the first group to report changes in blood flow following aerobic exercise with BFR. Specifically, compared to the LL condition blood flow during recovery was reduced by $\sim 33 \%$ and $\sim 50 \%$ in the BFR 60 and BFR 80 conditions, respectively. These results generally agree with previous literature analyzing reductions in blood flow following resistance exercise with BFR $(\sim 30-40 \%)[73,106]$. This reduction in blood flow to the muscle is supported by our tissue perfusion data. Specifically, tissue saturation index was reduced by $\sim 5 \%$ and $\sim 15 \%$ in the BFR 60 , and BFR 80 conditions throughout the condition. These reductions support previous reports on muscle oxygenation during cycling with BFR. Corvino and colleagues [34] reported $\sim 6 \%$ reduction in tissue saturation index during intermittent cycling with BFR, but only $\sim 2 \%$ reduction during continuous cycling with BFR. Therefore, the authors suggest that intermittent cycling with BFR may be more advantageous than continuous cycling with BFR. Our results support this conclusion by demonstrating that during the BFR conditions, tissue saturation index remained the same and metabolite accumulation increased during the recovery periods. Conversely, during the LL and HL conditions, tissue saturation increased and metabolite accumulation decreased during recovery. Without blood flow restriction, muscles experience a post-exercise hyperemic response, due to metabolite induced vasodilation [107]. Following exercise with BFR, cardiovascular control center is no longer receiving stimulus from mechanically sensitive afferents, thus reducing sympathetic activation. This in combination with the reduced venous return from venous occlusion and the loss of the skeletal muscle pump, means a relative reduction in blood flow to the limb following exercise. If the purpose of the 
occlusion cuff is to reduce muscle oxygenation and increase metabolite accumulation, then it is likely beneficial for cycling exercise with BFR to be performed intermittently with the cuff inflated during recovery, but additional research is needed to confirm this and how it would influence training adaptations.

\subsubsection{Metabolic, Cardiorespiratory, and Perceptual Responses}

Oxygen consumption did not change with the addition of BFR. Previous researchers $[6,108]$ reported an increase in $\mathrm{VO}_{2}$ during aerobic exercise with BFR likely due to the metabolic cost of increased heart rate and ventilation. Discrepancies in these findings may be due to the duration of the cycling intervals. Because the intervals were limited to 2 min, steady-state $\mathrm{VO}_{2}$ was not achieved. Heart rate, ventilation, RPE, pain, and lactate all increased with BFR and further increased with higher cuff pressure, which is consistent with previous research $[109,110]$. Notably, heart rate and RPE increased in the BFR 80 to a level that was not different from HL, additionally muscle pain during the last cycling interval was near maximum values.

\subsubsection{Neuromuscular Function}

Cycling exercise with BFR reduced MVIC torque by $\sim 18 \%$ and $\sim 40 \%$ in the BFR 60 , and BFR 80 conditions, respectively. These results generally agree with previous reports examining resistance exercise with BFR [31, 91-93], however, prior studies examining neuromuscular function following aerobic exercise with BFR have varied. Similar to resistance exercise reports $[31,91]$ we determined that these reductions were mainly due to peripheral mechanisms, especially in the BFR 60 condition. Exercise with 
BFR may have caused peripheral fatigue by the reduction in blood flow to the working muscles reducing the energy supply to the muscles, and/or by the accumulation of metabolites inhibiting cross-bridge formation [93].

In addition to peripheral fatigue, central fatigue was also present in the BFR 80 condition. Specifically, voluntary activation was $23 \%$ lower in the BFR 80 condition 1 min after exercise. This reduction in voluntary activation was notably larger than that of Hussmann et al. [93] and Karabulut et al. [92], who reported a $10 \%$ and $13 \%$ reduction in voluntary activation following resistance exercise with BFR, respectively. This reduction in voluntary activation in the BFR 80 condition could be due to several factors related to exercise with BFR. One explanation includes venous distension, and the accumulation of metabolites, both of which increase the firing rate of nociceptive group IV muscle afferents $[111,112]$. As stated above the BFR 80 condition resulted in significant muscle pain, which increased throughout the protocol. Graven-Nielsen and colleagues [113] previously reported muscle pain, via infusion of hypertonic saline, reduced MVIC torque through a centrally mediated mechanism. The results from the current study indicate substantial fatigue with the intermittent cycling protocol and provide the first report of central and peripheral fatigue with aerobic BFR.

\subsubsection{Cuff Pressure}

Proper selection of cuff pressure is important for the safety and effectiveness of exercise with BFR. Ideally, the cuff pressure should be set so that some arterial blood flow is maintained while venous blood flow is occluded. This causes a reduction in tissue perfusion and a build-up of metabolites and fluid within the limb. Previous authors have 
proposed these as possible mechanisms responsible for the training adaptations following exercise with BFR $[3,20]$. Higher cuff pressures likely result in greater venous occlusion which augments cell swelling by causing blood to pool within the limb. Loenneke and colleagues [26] suggested that the ideal cuff pressure necessary for training adaptation is less than previously used in the literature. This could be due to very high cuff pressures completely occluding arterial blood flow and thus attenuating the cell swelling response. Increasing cuff pressure also likely increases metabolite accumulation within the limb, which activate the exercise pressor reflex. The activation of the exercise pressor reflex and increased metabolite concentrations may explain the increased heart rate, ventilation, pain, and peripheral fatigue seen in the present study. These results may decrease BFR exercise training volume by early termination of the exercise. It should be noted that while BFR 80 resulted in a greater increases in metabolite accumulation it also resulted in substantial pain by the end of the final cycling interval (two participants could not complete the protocol). Therefore, this pressure was too high for the active male participants in the current study. Collectively, these results indicate that BFR 60 promotes lower metabolic, cardiorespiratory, and perceptual responses than traditional high-load cycling without inducing excessive pain, and therefore may be an acceptable cuff pressure for cycling with BFR.

\subsubsection{Implications}

The major findings of the present study were: 1) BFR caused a reduction in blood flow and tissue perfusion and augmented metabolite accumulation, 2) $\mathrm{VO}_{2}$, heart rate, ventilation, and RPE in the BFR 60 were lower than HL cycling, and 3) exercise with 
BFR impaired MVIC torque through peripheral and central mechanisms. These findings have implications for clinicians, practitioners, and researchers. Clinicians and practitioners should take great care in selecting cuff pressures as pressure effects acute and likely chronic responses to exercise. Cuff pressures of $\sim 60 \%$ limb occlusion pressure should be adequate for training as they result in reduced blood flow, decreased muscle oxygenation, increased metabolite accumulation, and reduced neuromuscular function, yet lower metabolic, cardiorespiratory, and perceptual responses than HL cycling. It is important to note that limb occlusion pressures vary by individual, cuff type, cuff width, and body position used $[40,43,50,51,114]$. Therefore cuff pressures should be based on individual limb occlusion pressure if possible. Using an absolute pressure may result in inconsistent training adaptations or adverse side-effects. Finally, this study highlights cuff pressures that elicit specific stresses to the neuromuscular systems that may be advantageous for cycling exercise with BFR, which may aid researchers in the development of robust cycling exercise with BFR guidelines (exercise protocols, cuff pressures) for healthy, clinical, and athletic populations.

\subsubsection{Limitations}

There are some limitations to our study that must be addressed. First, blood flow measurements using ultrasonography during cycling could not be performed, therefore we measured blood flow immediately (within 10 seconds) after each cycling interval. Although not directly measured, we can speculate that blood flow during exercise was higher than during recovery in the BFR conditions based on the tissue perfusion data. Second, our measurements of neuromuscular function were performed 1 min after the 
conclusion of exercise. This minute was used for the participant to transfer from the cycling ergometer to the isokinetic dynamometer. This time may have masked some of the changes to neuromuscular function following exercise. Moreover, changes in neuromuscular function are task-specific [95] and therefore caution must be taken when extrapolating these findings to other modes of aerobic exercise, exercise protocols, and/or other populations including women and older adults.

\subsubsection{Summary}

In summary, cycling with BFR decreased blood flow and tissue perfusion and increased metabolite accumulation when compared to LL cycling without BFR. Moreover, cycling with BFR resulted in lower metabolic and cardiorespiratory responses than traditional HL cycling, and reduced neuromuscular function by central and peripheral mechanisms. We conclude that intermittent cycling with a cuff pressure of $60 \%$ limb occlusion has the potential to strike a balance between increasing metabolite stress, which is needed for training adaptations, without causing excessive discomfort and cardiorespiratory strain. Therefore, cycling with this pressure may serve as an alternative exercise for individuals incapable of performing high-intensity exercise. 


\section{Table 3: Participant characteristics}

Height $(\mathrm{cm})$

Body mass $(\mathrm{kg})$

Age (yr)

Body fat (\%)

Lower limb lean mass $(\mathrm{kg})$

$\mathrm{VO}_{2}$ peak $(\mathrm{mL} / \mathrm{kg} / \mathrm{min})$

Heart rate peak (b/min)

LOP $(\mathrm{mmHg})$

Values are expressed as mean \pm SD. LLLM:

Lower limb lean mass. LOP: Limb occlusion pressure
$179 \pm 6$

$79 \pm 8$

$26 \pm 6$

$18 \pm 4$

$21 \pm 2$

$53 \pm 6$

$191 \pm 9$

$208 \pm 19$ 
A

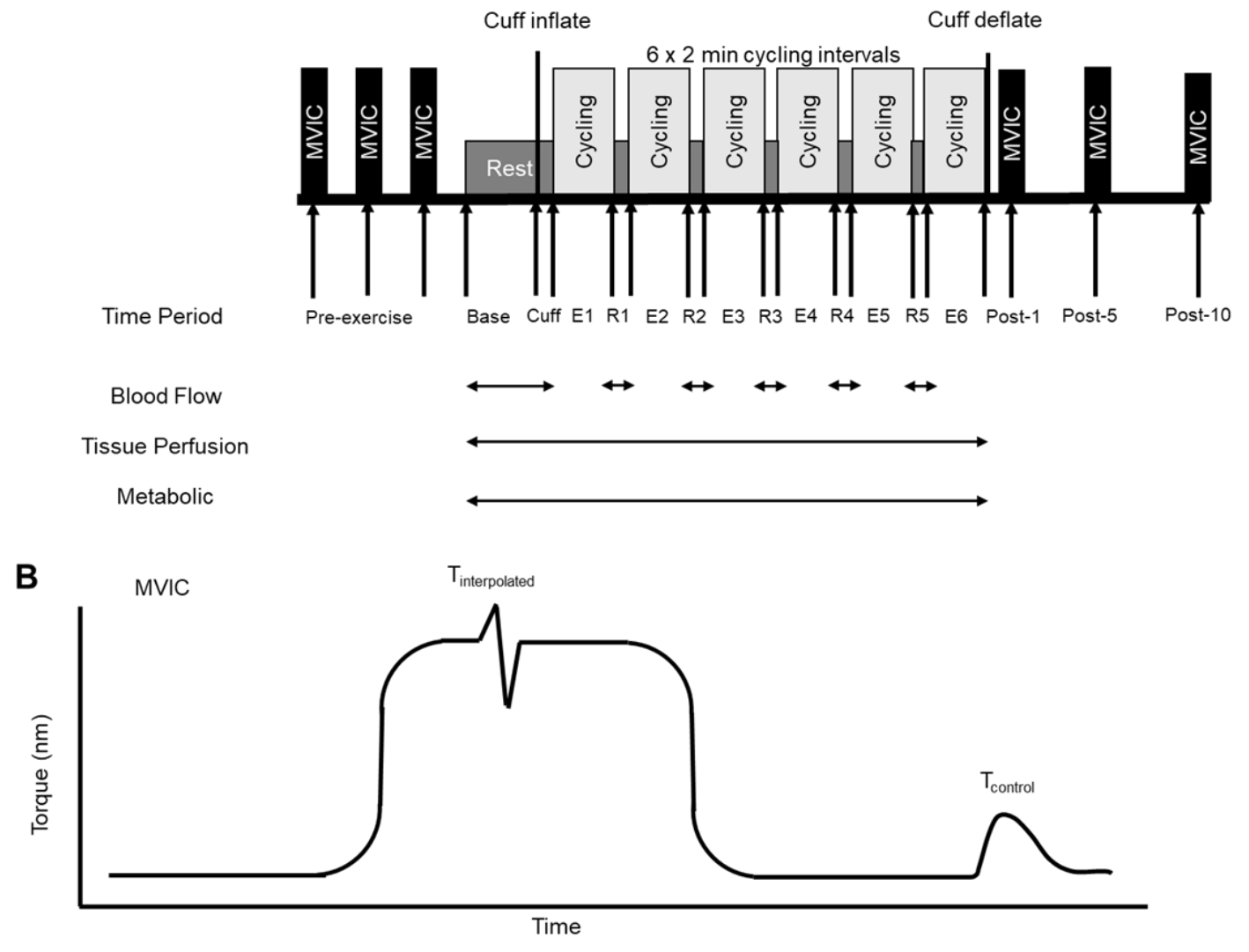

Figure 8: Overview of experimental cycling protocol. Base, Cuff, E, R, and Post-, denotes baseline, cuff inflate, exercise, recovery, and post-exercise periods, respectively. 


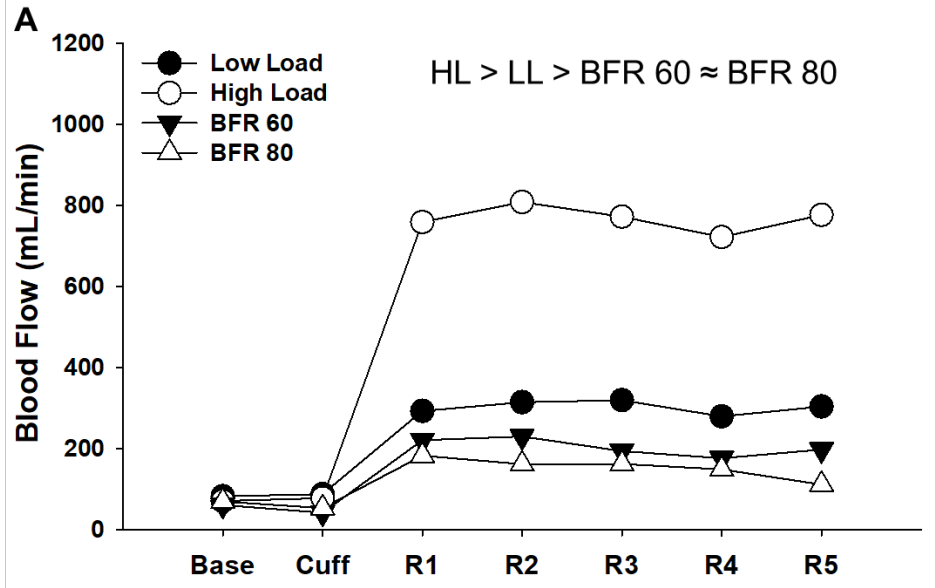

B

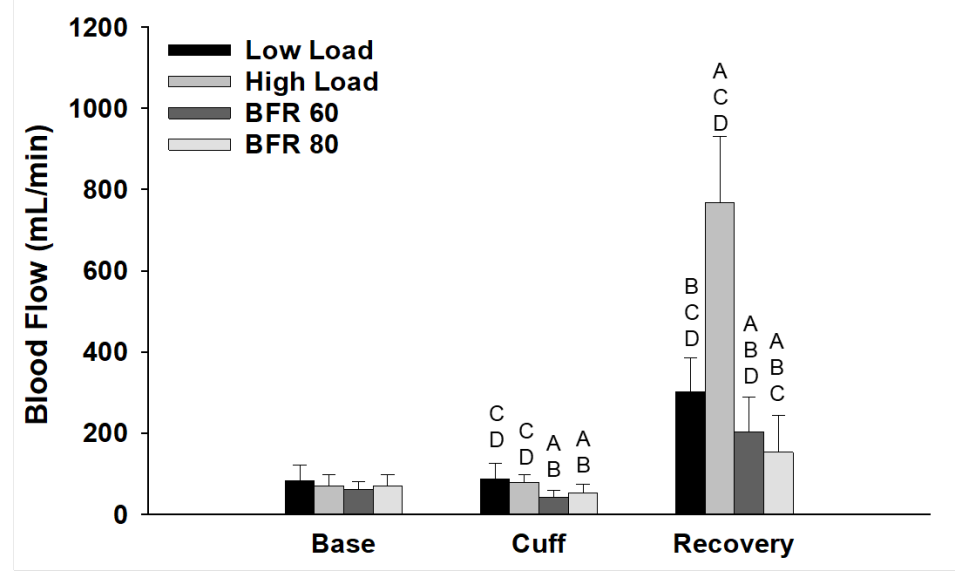

Figure 9: Alterations in superficial femoral artery blood flow across different conditions (A). Significant main effect of condition indicted in textbox above figure, $\approx(p>0.05),<$ or $>(p<0.05)$. Data reported as mean and standard deviation bars were removed for clarity. Mean superficial femoral artery blood flow during baseline, cuff inflate, and recovery periods (B). Blood flow different from LL, HL, BFR 60\%, and BFR 80\% (P< $0.05)$ are indicated by ${ }^{A},{ }^{B},{ }^{C},{ }^{D}$, respectively. Data are reported as mean \pm SD. 

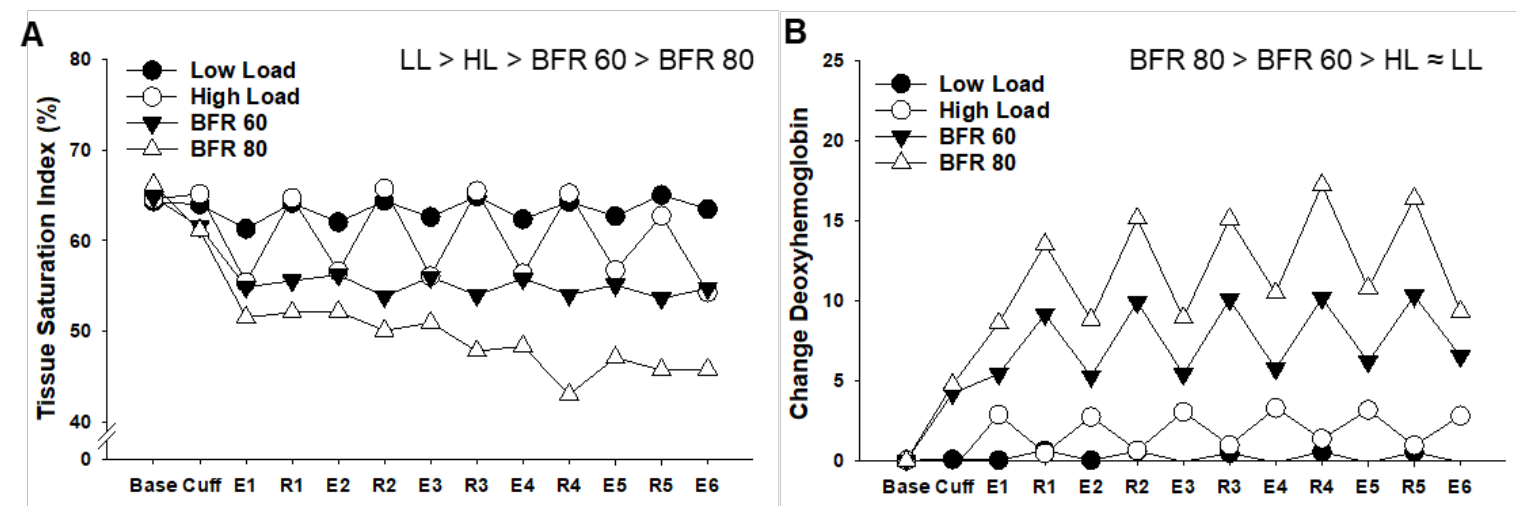

C

\section{D}
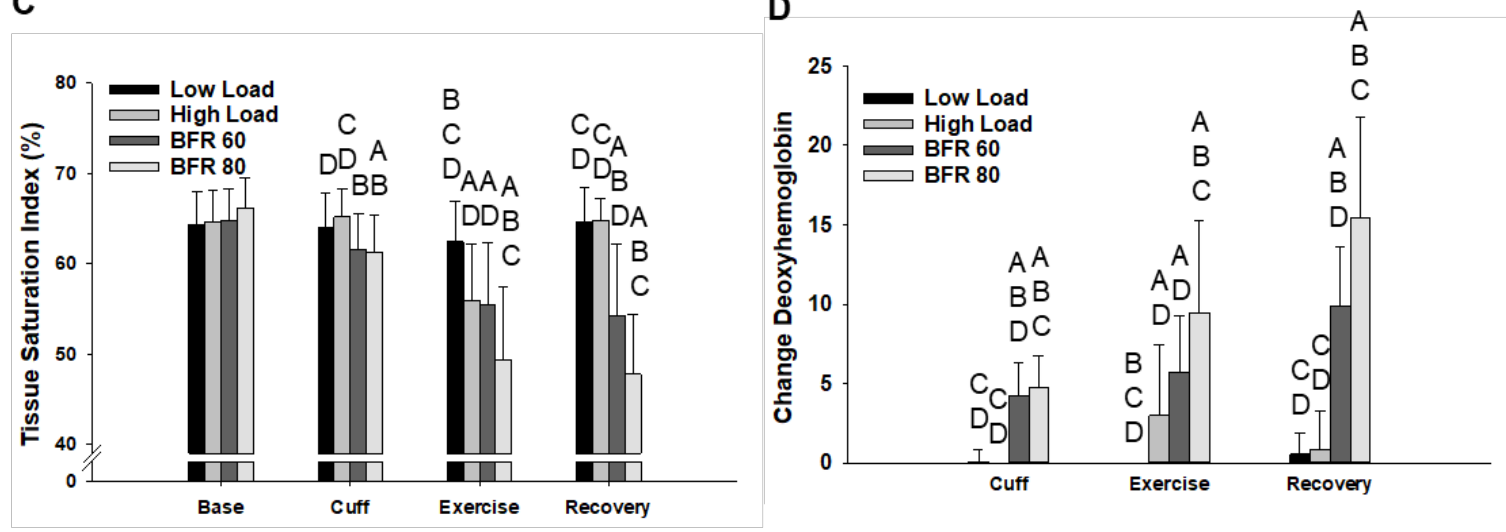

Figure 10: Changes in tissue saturation index (A) and concentrations of deoxyhemoglobin (B) throughout the cycling protocol. Significant main effect of condition indicted in textbox above individual figures, $\approx(\mathrm{p}>0.05),<$ or $>(\mathrm{p}<0.05)$. Data reported as mean, standard deviation bars were removed for clarity. Mean values for tissue saturation index (C) and concentrations of deoxyemoglobin (D) during baseline, cuff inflate, pooled exercise, and pooled recovery periods. Tissue saturation index and concentrations of deoxyhemoglobin different from LL, HL, BFR 60\%, and BFR $80 \%$ (P $<0.05$ ) are indicated by ${ }^{\mathrm{A}},{ }^{\mathrm{B}},{ }^{\mathrm{C}}, \mathrm{D}$, , respectively. Data are reported as mean $\pm \mathrm{SD}$. 

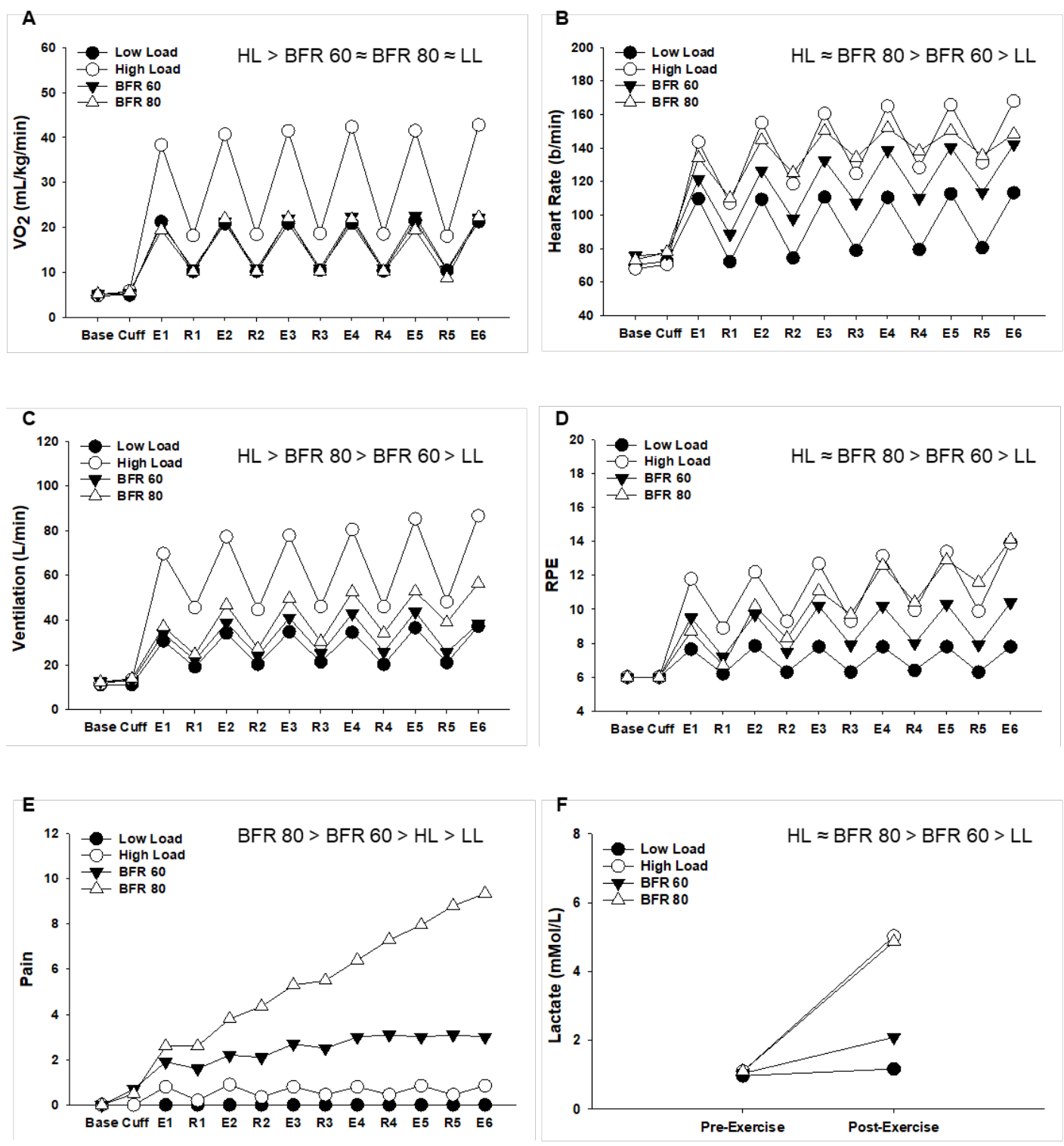

Figure 11: Time course alterations in $\mathrm{VO}_{2}(\mathrm{~A})$, heart rate (B), ventilation (C), $\mathrm{RPE}(\mathrm{D})$, pain $(E)$, and whole blood lactate $(F)$. Significant main effect of condition indicted in textbox above individual figures, $\approx(\mathrm{p}>0.05),<$ or $>(\mathrm{p}<0.05)$. Data reported as mean, standard deviation bars removed for clarity. 

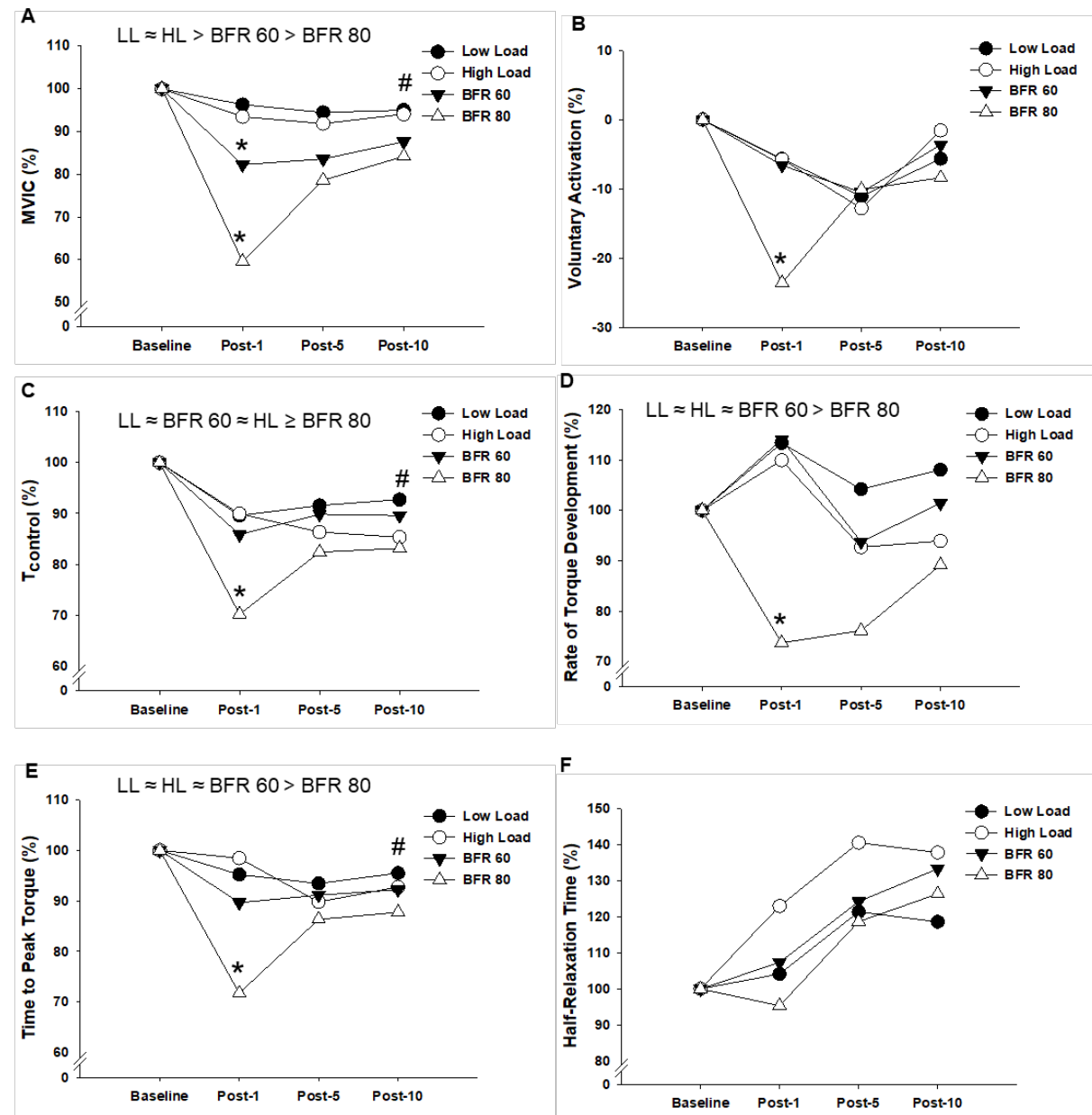

Figure 12: Maximal voluntary isometric contraction torque (MVIC), voluntary activation, evoked twitch torque ( $\left.\mathrm{T}_{\text {control }}\right)$, rate of torque development, time to peak torque, and half-relaxation time depicted in panel A, B, C, D, E, and F, respectively. Significant main effect of condition indicted in textbox above individual figures, $\approx$ (denotes $p>0.05),<$ or $>($ denotes $p<0.05)$. Note that, $T_{\text {control }} \geq$ indicated BFR 80 is different than LL and BFR 60, but not different than HL. Condition $x$ time interaction at (baseline to $1 \mathrm{~min}$ post-exercise) different than all other conditions $\left({ }^{*} \mathrm{P}<0.05\right)$. All conditions different from baseline post-10 (\# $\mathrm{P}<0.05$ ). Data reported as mean, standard deviation bars removed for clarity. 


\section{Adaptations to Low-Intensity Cycling and Low- Intensity Resistance Training with Blood Flow Restriction}

\subsection{Introduction}

Blood flow restriction (BFR) training is a safe and effective method of increasing both aerobic capacity and muscular function $[26,85,115]$ in healthy, clinical, and athletic populations (for extensive reviews see [26, 115-117]). In this exercise modality, a pressurized cuff or tourniquet is used to partially restrict blood flow into the limb in combination with resistance exercise, or aerobic exercise[23]. This pressurized cuff results in the reduction of oxygen delivery to the muscle and occludes venous return, which causes a build-up of metabolites thereby promoting muscle growth [88], microvascular filtration capacity [118], and angiogenesis [119]. Exercise training with BFR is of particular interest to researchers for its ability to increase muscular function and aerobic capacity at much lower intensities than those traditionally used in exercise training, making this modality especially beneficial for those populations who are unable to perform high intensity exercise (e.g., individuals with orthopedic injuries, and older adults).

Because of exercise with BFR's unique ability to stimulate muscular adaptations at low training loads, many exercises including walking and cycling that traditionally would not improve muscle function can be effectively used with BFR to increase muscle size and strength. 
Although aerobic and resistance training with BFR have not been directly compared, two separate meta-analyses have suggested resistance training with BFR would increase muscular size and strength to a greater degree than walking with BFR $[26,115]$. Loenneke and colleagues [26] reported effect sizes of Cohen's $d=0.44$, and 0.31 for increased muscular strength in resistance and aerobic exercise with BFR respectively. Additionally, Slysz and colleagues [115] reported typical increases in strength of $0.3 \mathrm{~kg}$, for resistance training and $0.4 \mathrm{Nm}$ for aerobic training. Alternatively, only aerobic exercise with $\mathrm{BFR}$ has been reported to increase aerobic capacity $\left(\mathrm{VO}_{2 \max }\right.$, time until exhaustion, onset of blood lactate) $[10,36-38,86]$. Therefore, while muscle hypertrophy might be greater in resistance training with BFR, aerobic exercise with BFR may serve as a 2-for-1 activity in which participants increase both muscle function and aerobic capacity. Additionally, recent evidence also suggests that aerobic exercise with BFR elicits lower cardiovascular responses (e.g., heart rate, cardiac output, mean arterial pressure) $[35,39]$ and reduced perceived effort than resistance exercise with BFR [35, 39] With these factors in mind, several authors [35, 39] have suggested that aerobic exercise with BFR is more tolerable.

To date, there has not been a comparison of chronic training adaptations following aerobic and resistance training within equivalent matched groups. This information would allow clinicians and practitioners to make more informed decisions on the type of exercise to perform with BFR. Therefore, the purpose of this study was to evaluate changes in muscular function and aerobic capacity following 6 weeks of either aerobic or resistance exercise with BFR. We hypothesized that both resistance training 
and aerobic training with BFR would increase muscle size and strength, but resistance BFR training would result in greater muscular adaptions [26, 115]. We also hypothesized that aerobic training with BFR would result in greater increases in aerobic capacity compared to resistance training with BFR.

\subsection{Methods}

\subsubsection{Participants}

Eighteen healthy adults between 18-44 years of age volunteered to participate in this study (demographic and anthropometric characteristics reported in Table 4).

Participants were excluded from the study if they used nicotine products, had diabetes, or had any cardiopulmonary disorders. None of the participants self-reported that they performed regular physical activity for $30 \mathrm{~min}$ at moderate intensity on at least 3 days per week in the last 3 months, which is consistent with the American College of Sports Medicine's definition of sedentary [120]. Female participants were excluded if they were using any hormonal contraceptives. Additionally, none of the participants had performed exercise with BFR on a regular basis. Following the initial screening, participants were informed of the purpose of the study, the risks involved, and gave informed written consent. This study was approved by the Institutional Review Board at Michigan Technological University.

\subsubsection{Study Overview}

Participants were assigned to one of two exercise groups: aerobic or resistance training. Groups were matched on demographics, body composition, muscular function, 
and aerobic capacity characteristics. Both groups performed BFR exercise $3 \mathrm{x} /$ wk for 6 weeks. Training outcome measures of muscle size (lower limb lean mass, muscle thickness), muscle strength (isometric strength, knee extension estimated 1 repetition max, leg press estimated 1 repetition max $)$, and aerobic capacity $\left(\mathrm{VO}_{2 \text { peak, lactate }}\right.$ threshold, peak aerobic power) were performed prior to training and within 10 days after completion of the training program (Figure 1). Note that, pre-training and post-training measures for female participants, were collected during the follicular phase of their menstrual cycle.

\subsubsection{Blood Flow Restriction}

During exercise, femoral blood flow was restricted using a $10 \mathrm{~cm}$ wide nylon pneumatic cuff (SC10D, Hokanson, Belleview, WA, USA) wrapped around each thigh at the most proximal location. The cuff was pressurized with a rapid cuff inflator (Hokanson, Belleview, WA, USA). The training cuff pressure was set at $60 \%$ of individual limb occlusion pressure and monitored throughout the training session. To determine the limb occlusion pressure, participants were placed in a seated position during which blood flow velocity was assessed. Specifically, blood flow velocity was measured with a Doppler ultrasound system (Logiq e BT12, GE Healthcare, Chicago, IL, USA) equipped with a linear array transducer operating at an imaging frequency of 12 $\mathrm{MHz}$ and Doppler frequency of $5 \mathrm{MHz}$. The ultrasound probe was positioned just distal to the cuff over the superficial femoral artery. The angle of insonation of the ultrasound probe was always $\leq 60^{\circ}$. Limb occlusion pressure was identified by inflating the cuff to $75 \mathrm{mmHg}$, and slowly increasing the pressure until blood flow velocity reached zero, 
based on the absence of the Doppler spectrum. The minimum pressure was recorded as the limb occlusion pressure. Pilot data from our laboratory indicated that the measurement of LOP using this method was stable across days $(\mathrm{ICC}=0.97)$; which is consistent with previous literature [50].

\subsubsection{Aerobic Training}

Each exercise session consisted of multiple 2 min intervals on a cycle ergometer (Velotron Elite, RacerMate Inc., Seattle, WA, USA). In weeks 1 and 2, participants completed 6 total cycling intervals. In weeks 3-6, participants completed 8 intervals. The work rate was $40 \%$ of the participant's peak aerobic power measured during a graded exercise test. Pedaling rate was self-selected. Participants were given 1 min rest between intervals and the pressure in the cuffs was maintained during these rest periods. Following the $3^{\text {rd }}$ and $4^{\text {th }}$ interval for weeks 1 and 2 , and 3-6 respectively, the cuff was deflated and the participants were given 2 min rest.

\subsubsection{Resistance Training}

Each exercise session consisted of dynamic bilateral leg press (GZFH8043, FreeMotion, Logan, UT, USA) and knee extension (C8080W, New York Barbells, Elmira, NY, USA) exercise. In weeks 1 and 2 participants completed 3 sets of each exercise, and in weeks 3-6 participants completed 4 sets of each exercise. Participants performed 30 repetitions in the first set and 15 repetitions in all subsequent sets. Intensity was $30 \%$ of the participant's estimated 1RM. Repetitions were set to a metronome and were performed $1.33 \mathrm{~s}$ for the concentric phase and $1.33 \mathrm{~s}$ for the eccentric phase. 
Participants were given 1 min rest between sets, and 2 min rest between exercises. The pressure in the cuffs was maintained between sets but deflated between exercises.

\subsubsection{Training Measures}

During each training session, ratings of perceived exertion, pain, and heart rate were recorded during the last $15 \mathrm{~s}$ of exercise and rest. Whole body rating of perceived exertion was assessed using a Borg 6-20 scale [100]. Pain was assessed on an 11 point numeric rating scale [101]. Heart rate was measured continuously using a Polar transmitter (Polar Electro OY, Kempele, Finland). Mean arterial pressure was recorded during each rest period using an automated sphygmomanometer (Omron HEM-907XL, Omron Health Care Inc., Lake Forest, IL, USA). Before each training session participants performed a body weight half squat to a knee angle of $45^{\circ}$ to assess the level of perceived muscle soreness in their legs. Participants were asked to place a mark on a $10 \mathrm{~cm}$ visual analog scale [121]. This scale was anchored by written cues at $0 \mathrm{~cm}$ (no soreness) and 10 $\mathrm{cm}$ (very severe soreness). Muscle soreness was quantified by measuring the distance to the mark.

\subsubsection{Training Outcome Measures}

\subsubsection{Body Composition}

Regional body composition was assessed using dual energy x-ray absorptiometry (Discovery Wi, Hologic Inc, Marlborough, MA, USA). Lower limb lean mass, lower limb fat mass, and body fat percentage were recorded. The coefficient of variation of this 
system for the measurement of lean, and fat mass has been reported to be $0.6-1.4 \%$, and $1.8-2.1 \%$ respectively $[122,123]$.

\subsubsection{Muscle Thickness}

Muscle thickness of the right rectus femoris and vastus lateralis were measured using a b mode ultrasound (Logiq e BT12, GE Healthcare, Chicago, IL, USA) equipped with a linear array transducer operating at an imaging frequency of $12 \mathrm{MHz}$ and Doppler frequency of $5 \mathrm{MHz}$. Participants were positioned supine with their legs resting comfortably with about 10 degrees of knee flexion. A rolled towel was placed under the knee for support. Images of the rectus femoris (RF) and vastus lateralis (VL) were taken at $50 \%$ of the distance from the anterior superior iliac spine to the proximal patella. Muscle thickness was measured as the distance between the superficial and deep aponeurosis at the widest distance. Measures of muscle thickness were taken on 2 days, separated by at least $24 \mathrm{hrs}$. Five images were taken per muscle per day, and the average of the 10 closest measurements was used for analysis. If the average of the 2 days differed by more than $2.5 \%$, muscle thickness measurements were taken on an additional day. The same investigator performed all the ultrasound measurements. The intraclass correlation coefficient for assessing the reliability of the RF and VL muscle thickness measurements across days was 0.995 (95\% confidence interval: 0.982-0.999); 0.998 (95\% confidence interval: $0.994-1.000)$ with a coefficient of variation of $1.4 \%$, and $1.1 \%$ for the RF and VL respectively. 


\subsubsection{Muscular Strength}

Isometric knee extension strength was determined using a force transducer (MLP300, Transducer Techniques, Temecula, CA, USA). Knee extension forces were recorded using a data acquisition system (PowerLab 16, ADInstruments, Colorado Springs, CO, USA). Participants were seated with a hip angle of $\sim 85^{\circ}$, and a knee angle of $\sim 90^{\circ}$. To measure isometric strength, participants were asked to "push as hard and as fast as possible" for $\sim 5 \mathrm{~s}$. Standardized verbal encouragement was provided to the participant. Measures of isometric knee extension strength were taken on 3 separate days, separated by at least $24 \mathrm{hrs}$. Five maximal voluntary contractions were taken per day. The average force of the 2 highest measurements within 5\% was calculated and isometric strength to body mass ratio was recorded. The intraclass correlation coefficient of knee extension force across days was 0.968 (95\% confidence interval: $0.916-0.990)$ with a coefficient of variation of $6.7 \%$.

Estimated 1 RM was determined on a leg press (GZFH8043, FreeMotion, Logan, UT, USA) and knee extension machine (C8080W, New York Barbells, Elmira, NY, USA). The participant completed 10-15 repetitions of weight $\sim 50 \%$ of the individual's 1 RM as a warm-up. Subjects were given a 2 min rest and asked to complete 10 repetitions of $\sim 80 \%$ their estimated $1 \mathrm{RM}$. If the participant could successfully complete 10 repetitions with proper form, $4.5 \mathrm{~kg}$ was added and the participant attempted another 10 repetitions following a $2 \mathrm{~min}$ rest. This was repeated until the subject could no longer perform 10 repetitions with proper form. The weight and number of repetitions completed were recorded and 1RM was estimated using the Brzycki equation. $1 \mathrm{RM}=\mathrm{w}$ 
$\mathrm{x}(36$ / (37-r)) [124], where $\mathrm{w}$ is the weight and $\mathrm{r}$ is the number of repetitions. Estimation of knee extension and leg press 1 RM using this method has been previously reported as reliable in untrained men and women [125]. Leg press and knee extension strength was normalized to body weight.

\subsubsection{Aerobic Capacity}

Participants performed a graded exercise test on an electromagnetically-braked cycle ergometer (Velotron Elite, RacerMate Inc., Seattle, WA, USA) until task failure to

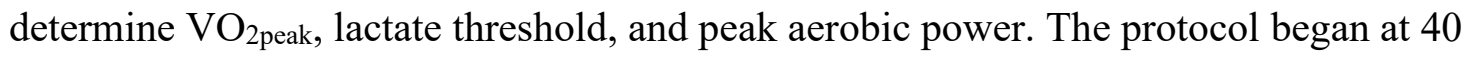
$\mathrm{W}$ and increased 35 or $25 \mathrm{~W}$ every 3 min for men and women respectively. The pedaling rate was self-selected. The test was terminated voluntarily by the subject or when pedaling rate could no longer be maintained above $70 \mathrm{rpm}$ despite verbal encouragement. Peak aerobic power $\left(\mathrm{W}_{\text {peak }}\right)$ was interpolated based on the time complete in the terminal stage [126]. During the test, breath by breath gas exchange data was collected using a metabolic measuring system (True Max 2400, Parvo Medics, Sandy, UT, USA). The metabolic measurement system was calibrated with a 3L syringe and medical gases of known concentrations $\left(16.00 \% \mathrm{O}_{2}, 4.00 \% \mathrm{CO}_{2}\right.$, balanced $\left.\mathrm{N}_{2}\right)$. Gas exchange data were continuously recorded and averaged every $30 \mathrm{~s}$. The highest $30 \mathrm{~s} \mathrm{VO}_{2}$ value was recorded

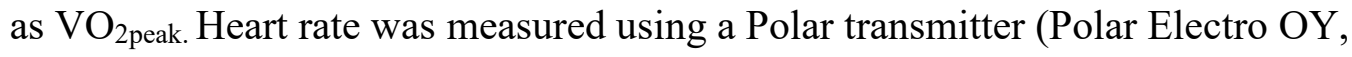
Kempele, Finland). A blood sample was collected from the fingertip $(5 \mu \mathrm{l})$ during the final min of each stage from which blood lactate concentration was determined (Lactate Pro LT-1710; ARKRAY, Kyoto, Japan). Blood lactate concentrations were plotted against power. The $\mathrm{D}_{\max }$ lactate threshold was determined by calculating the power output 
that corresponded to the greatest perpendicular distance from a third-order polynomial curve and a straight line formed by the first and last data points[127, 128].

\subsubsection{Statistical Analysis}

Separate 2 (aerobic vs. resistance) x 2 (pre-training vs. post-training) mixed repeated measures analysis of variance procedures (ANOVA) were used to assess differences in muscular function (muscle mass, muscle thickness, muscle strength) and aerobic capacity $\left(\mathrm{VO}_{2 \text { peak, }}\right.$ Wpeak, Lactate Threshold) values. Separate 2 (aerobic vs. resistance) x 6 (weeks of training) mixed repeated measures ANOVA procedures were performed on rating of perceived exertion, pain, heart rate, mean arterial pressure, and muscle soreness. If the ANOVA revealed a significant interaction, then subsequent independent t-tests were used to identify where the groups differed. Partial eta squared $\left(\eta p^{2}\right)$ was calculated as a measure of effect size with $\eta p^{2} \geq 0.01$ indicating small, $\geq 0.059$ medium, and $\geq 0.138$ large effects, respectively [105]. Statistical procedures were performed using SPSS 22 (Armonk, NY, USA). Data are reported as mean \pm SD and alpha was set to 0.05 .

\subsection{Results}

\subsubsection{Pre-Training}

There were no significant differences in anthropometric measures (age, height, body mass, thigh circumference), muscular function (muscle size, strength), aerobic capacity $\left(\mathrm{VO}_{2 \text { peak }}\right.$, lactate threshold, peak aerobic power) or body composition (body fat 
percentage, lower limb lean thigh mass, lower limb fat percentage) between groups (all $\mathrm{P}$ $>0.05$; Table 4).

\subsubsection{Blood Flow Restriction Training}

Participants completed $99 \%$ of the total training sessions as three training sessions were missed due to scheduling conflicts. One participant from the aerobic group did not complete the training due to scheduling conflicts, thus 8 subject were used in the aerobic group were used for analysis. Mean exercise heart rate throughout training in the aerobic group $(114 \pm 5 \mathrm{bpm})$ and resistance group $(120 \pm 5 \mathrm{bpm})$ were not different $\left(\mathrm{P}=0.46, \eta \mathrm{p}^{2}\right.$ $=0.04$; Figure 14). Throughout training, MAP measured during the rest periods was also not different between the aerobic and resistance groups $(96 \pm 3 \mathrm{mmHg}$ vs. $103 \pm 3$ $\mathrm{mmHg}$, respectively; $\mathrm{P}=0.19, \eta \mathrm{p}^{2}=0.12$; Figure 2). Rating of perceived exertion and pain/discomfort throughout training were higher in the resistance group compared to the aerobic group $\left(\mathrm{P}=0.02, \eta \mathrm{p}^{2}=0.32 ; \mathrm{P}<0.01, \eta \mathrm{p}^{2}=0.44\right.$ respectively; Figure 14$)$. Muscle soreness that resulted from aerobic and resistance training was minimal $(0.2 \pm 0.1 \mathrm{~cm}$ vs. $0.6 \pm 0.1 \mathrm{~cm}$, respectively), but there was a trend for slightly higher muscle soreness in the resistance group $\left(\mathrm{P}=0.08, \eta \mathrm{p}^{2}=0.19\right)$.

\subsubsection{Muscular Function}

\subsubsection{Body composition:}

In general, BFR training regardless of group caused an increase in lower limb lean mass, and a decrease in body fat percentage. Specifically, a main effect of training on lower limb lean mass $\left(\mathrm{P}<0.01, \eta \mathrm{p}^{2}=0.55\right)$ was found, but no group $\mathrm{x}$ training 
interaction $\left(P=0.81, \eta p^{2}=0.81\right.$; Figure 15$)$. Similarly, for body fat percentage there was a significant main effect of training $\left(\mathrm{P}<0.01, \eta p^{2}=0.40\right)$, but no group $\mathrm{x}$ time interaction $\left(\mathrm{P}=0.42, \eta \mathrm{p}^{2}=0.04\right)$. Finally, lower limb fat mass did not change with training $(\mathrm{P}=$ $\left.0.22, \eta p^{2}=0.10\right)$

\subsubsection{Muscle Thickness:}

Both aerobic and resistance training increased muscle function to a similar extent. Training effects were found for RF and VL thickness $\left(\mathrm{P}<0.01, \eta \mathrm{p}^{2}=0.44 ; \mathrm{P}<0.01, \eta \mathrm{p}^{2}\right.$ $=0.72$ respectively $)$, but no training $x$ group interactions $\left(P=0.70, \eta p^{2}=0.01 ; P=0.18\right.$, $\eta p^{2}=0.12$ respectively; Figure 15$)$

\subsubsection{Muscle strength:}

Similarly to muscle thickness, strength to body mass ratio, leg press $1 \mathrm{RM}$, and knee extension $1 \mathrm{RM}$, increased with training $\left(\mathrm{P}<0.01, \eta \mathrm{p}^{2}=0.44, \mathrm{P}<0.01, \eta \mathrm{p}^{2}=0.42\right.$, $\mathrm{P}<0.01, \eta \mathrm{p}^{2}=0.82$ respectively), this increase not differ between groups. $\left(\mathrm{P}=0.93, \eta \mathrm{p}^{2}\right.$ $<0.01 ; \mathrm{P}=0.87, \eta p^{2}<0.01 ; \mathrm{P}=0.12, \eta \mathrm{p}^{2}=0.16$ respectively; Figure 16)

\subsubsection{Aerobic Capacity}

For $\mathrm{VO}_{2 \text { peak }}$, the ANOVA revealed a training $\mathrm{x}$ group interaction $\left(\mathrm{P}=0.02, \eta \mathrm{p}^{2}=\right.$ $0.30)$, but no main effect of training $\left(\mathrm{p}=0.12, \eta \mathrm{p}^{2}=0.15\right)$. There was a $5 \%$ improvement in $\mathrm{VO}_{2 \text { peak }}$ in the aerobic group $(\mathrm{P}=0.02)$, but no change in the resistance group $(\mathrm{P}=$ 0.54, Figure 17). For lactate threshold and peak aerobic power, no main effects of training $\left(\mathrm{P}=0.11, \eta \mathrm{p}^{2}=0.16, \mathrm{P}=0.19, \eta \mathrm{p}^{2}=0.11\right.$ respectively $)$ or training $\mathrm{x}$ group interactions $\left(\mathrm{P}=0.95, \eta \mathrm{p}^{2}<0.01, \mathrm{P}=0.22, \eta \mathrm{p}^{2}=0.64\right.$ respectively) were found. 


\subsection{Discussion}

We reported, for the first time, similar increases in muscle size and strength between aerobic and resistance training with BFR. Not only did aerobic training with BFR improve muscle size and strength, but also increased $\mathrm{VO}_{2 \text { peak. }}$ Moreover, aerobic training was performed at a lower rating of perceived exertion and resulted in lower muscle pain during exercise. Collectively, these data suggest that aerobic exercise with BFR may be the better method for training in populations who are unable to perform high-intensity exercise.

\subsubsection{Exercise Training Measures}

Previous research reported lower RPE during treadmill walking with BFR when compared to leg press exercise with BFR $[35,39]$. Our data support and extend upon these findings by corroborating their RPE findings and also demonstrating reduced pain in the aerobic group. Specifically, RPE during exercise was rated as 8.6 or "very light" in the aerobic group, and 11.0 or "light" in the resistance group. Throughout training, pain was rated at 1.7 or "mild pain" in the aerobic group and 3.7 or "moderate pain" in the resistance group. Moreover, although minimal, there was a trend for reduced muscle soreness in the aerobic group. Exercise training resulted in similar heart rates and blood pressures between groups. These results are contrary to previous research $[35,39]$ which demonstrated reduced heart rate and blood pressures during acute treadmill walking compared to leg press exercise. These discrepancies are likely due to differences in exercise modality and intensity. According to a recent questionnaire on the use of exercise with BFR, 99 out of 115 practitioners reported using resistance training, while 
only 22 reported using aerobic training [23]. Results from the current study demonstrate clear advantages for performing aerobic training, and therefore practitioners considering implementing exercise training with BFR should use aerobic exercise (walking or cycling) if possible.

\subsubsection{Muscular Size and Strength}

Contrary to our hypothesis, aerobic and resistance training with BFR resulted in similar increases in muscle size and strength. Specifically, lower limb lean mass, RF thickness, and VL thickness increased by $3.9,4.9$, and $7.0 \%$, respectively in both aerobic and resistance training groups. Additionally, isometric knee extension torque, as well as estimated knee extension and leg press $1 \mathrm{RM}$, improved by $8.2,14.5$, and $7.5 \%$ respectively in both groups. These improvements fall within the range of previously reported increases in muscle size and strength following aerobic and resistance training with BFR. For example, Abe and colleagues [129] demonstrated that walking with BFR increased quadriceps muscle volume by $4.1 \%$ and muscle strength by $10.4 \%$ in young, healthy men. In related work, Abe and coworkers [10] reported a 5.1\%, and 7.7\% increase in quadriceps muscle volume and knee extension strength following cycling training with BFR respectively. Similar increases in muscle size are reported following resistance training. Fujita and colleagues [7] documented a 3.0\% increase in quadriceps muscle volume and a $6.7 \%$ increase in knee extension strength following resistance training with BFR. Yasuda and coworkers [130] similarly reported a 7.8\% and $14.0 \%$ increase in quadriceps muscles size and squat $1 \mathrm{RM}$ following squat training with BFR. Although the exact mechanism for hypertrophy following exercise with BFR is unknown, 
some proposed mechanisms include accelerated fatigue leading to preferential type II fiber recruitment [18], augmented metabolite accumulation and local hypoxia [19], and cell swelling [131]. Moreover, there may be an endocrine response as exercise with BFR increases serum growth hormone to a greater extent than traditional high-load resistance training [21]. It is thought that this increase in growth hormone secretion occurs through the stimulation of chemoreceptors due to decreases in muscle perfusion [22]. Aerobic and resistance training with BFR similarly increase muscular function, this result has important implications for rehabilitation programs in which high-load resistance training is contraindicated.

\subsubsection{Aerobic Capacity}

There was a small but significant improvement in $\mathrm{VO}_{2 \text { peak }}$ for the aerobic group

but $\mathrm{VO}_{2 \text { peak }}$ did not change in the resistance group. Specifically, $\mathrm{VO}_{2 \text { peak }}$ improved by $4.5 \%$ following cycling training with BFR. It should be noted that the day-to-day variation of $\mathrm{VO}_{2 \text { peak }}$ has been perviously reported to range from $3-5 \%[132,133]$. The increase in $\mathrm{VO}_{2 \text { peak }}$ in the present study is similar in magnitude to other reports following cycling training with BFR. Specifically, de Oliveira and colleagues [38] demonstrated a $6.1 \%$ increase in $\mathrm{VO}_{2 \text { peak }}$ following 4 weeks of cycling with BFR and Abe and coworkers [36] reported a $6.4 \%$ increase in $\mathrm{VO}_{2 \text { peak }}$ following 8 weeks of cycling with BFR. To the best of our knowledge, there are no reports documenting improved aerobic capacity following resistance training with BFR. There is a lack of research examining the cellular mechanism for improved aerobic capacity following aerobic exercise with BFR, however, previous reports on supine leg cycling in a pressurized chamber have 
demonstrated increased muscle oxidative enzyme activity and capillary density [134, 135]. As this method also reduces blood flow to the working muscle, it may provide some insight into changes in aerobic capacity following cycling with BFR. These results extend upon previous research by demonstrating that aerobic exercise with BFR serves as a 2-for-1 activity, in which participants increase both muscle function and aerobic capacity. These results are especially important to clinicians working with populations that are unable to perform traditional exercise training or populations that can benefit from increases in both muscle function and aerobic capacity (e.g. older adults).

\subsubsection{Limitations}

One limitation of the present study was total work and time under occlusion were not matched between groups. The training protocols we chose were selected because they are commonly used in research and in practice [23], and were therefor chosen to maintain as much external validity as possible. Training adaptations to exercise with BFR are heavily influenced by intensity, duration, cuff pressure, and exercise volume. As such, care must be taken when extrapolating these findings to other exercise protocols. Finally, it is important to note that participants in the present study were young untrained and otherwise healthy. While no adverse side effects were reported in the presented study, caution should be taken when prescribing exercise with BFR to clinical populations, especially those with an exaggerated sympathetic response to exercise (e.g. those hypertension, heart failure, or peripheral artery disease) [82]. 


\subsubsection{Summary}

In summary, the purpose of this study was to compare changes in muscular function and aerobic capacity following $6 \mathrm{wks}$ of aerobic and resistance training with BFR. We found similar increases in muscle size and strength in aerobic and resistance training, and an increase in $\mathrm{VO}_{2 \text { peak }}$ in the aerobic group only. Moreover, aerobic training with BFR resulted in lower perceived effort and muscle pain during exercise. These improvements were achieved at much lower exercise intensities than what is generally recommended for aerobic and resistance training. We conclude that while both aerobic and resistance training with BFR are equally effective at increasing muscle size and strength, aerobic exercise has additional benefits (increasing aerobic capacity, lower effort, and reduced pain) and therefore may be the better option for exercise training with BFR. 


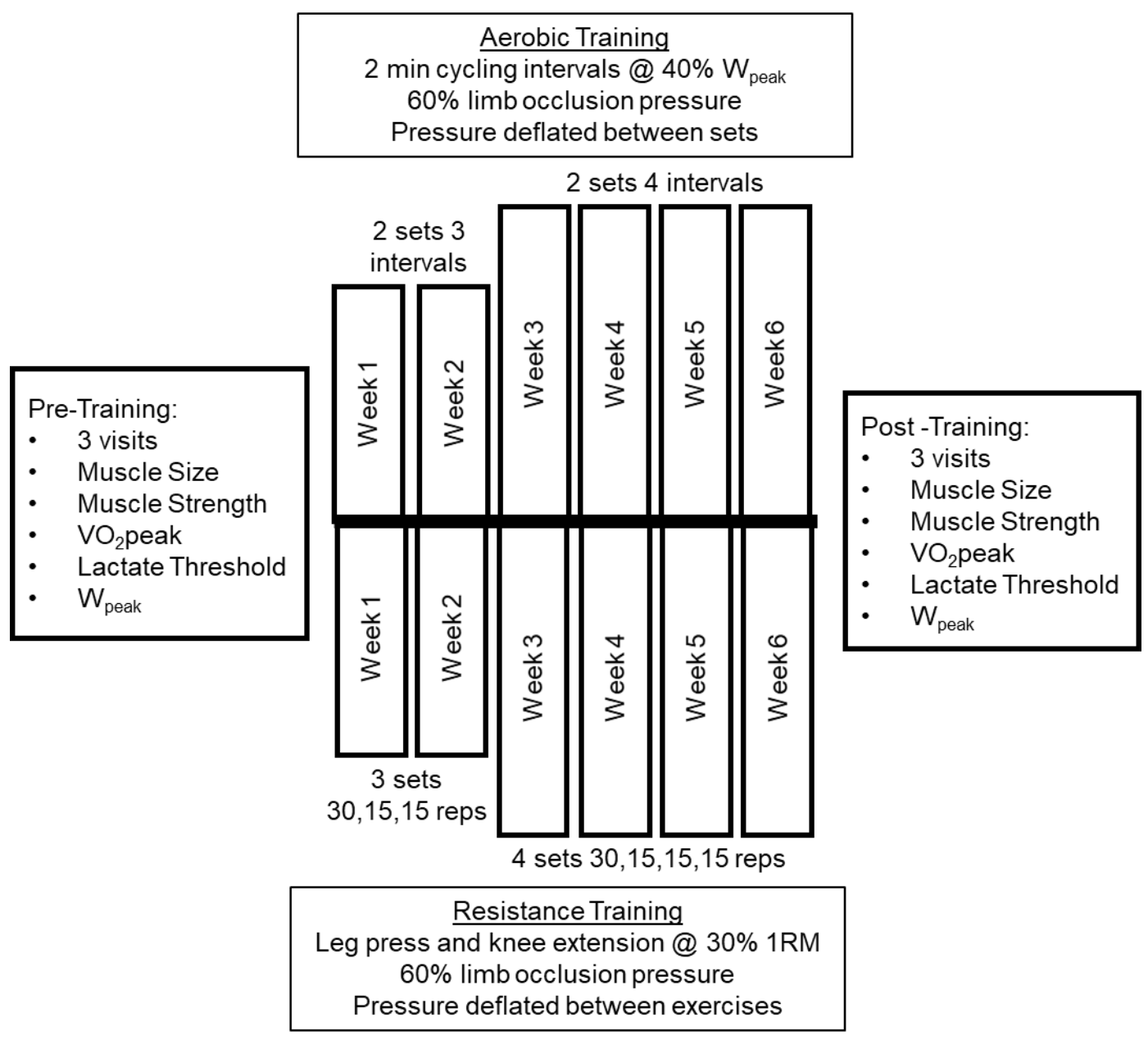

Figure 13: Overview of aerobic and resistance training protocol. 
Table 4: Pre-training subject characteristics

\begin{tabular}{|c|c|c|c|}
\hline & Aerobic & Resistance & P-value \\
\hline $\begin{array}{l}\text { Total participants } \\
\text { (n) }\end{array}$ & 9 & 9 & $\mathrm{n} / \mathrm{a}$ \\
\hline Female $(n)$ & 3 & 4 & $\mathrm{n} / \mathrm{a}$ \\
\hline Male $(n)$ & 6 & 5 & $\mathrm{n} / \mathrm{a}$ \\
\hline Age $(y r)$ & $25 \pm 5$ & $27 \pm 7$ & 0.45 \\
\hline Height $(\mathrm{cm})$ & $176 \pm 10$ & $174 \pm 9$ & 0.63 \\
\hline Body mass (kg) & $83 \pm 19$ & $81 \pm 12$ & 0.81 \\
\hline $\begin{array}{l}\text { Thigh } \\
\text { circumference }(\mathrm{cm})\end{array}$ & $59 \pm 9$ & $60 \pm 4$ & 0.80 \\
\hline $\begin{array}{l}\text { Limb occlusion } \\
\text { pressure }(\mathrm{mmHg})\end{array}$ & $189 \pm 34$ & $205 \pm 20$ & 0.25 \\
\hline Body fat (\%) & $28 \pm 11$ & $30 \pm 8$ & 0.53 \\
\hline $\begin{array}{l}\text { Lower limb lean } \\
\text { mass }(\mathrm{kg})\end{array}$ & $19 \pm 4$ & $18 \pm 3$ & 0.71 \\
\hline KE 1 RM & $142 \pm 41$ & $141 \pm 37$ & 0.95 \\
\hline $\mathrm{VO}_{2 \text { peak }}(\mathrm{ml} / \mathrm{kg} / \mathrm{min})$ & $35 \pm 8$ & $34 \pm 8$ & 0.81 \\
\hline
\end{tabular}


A

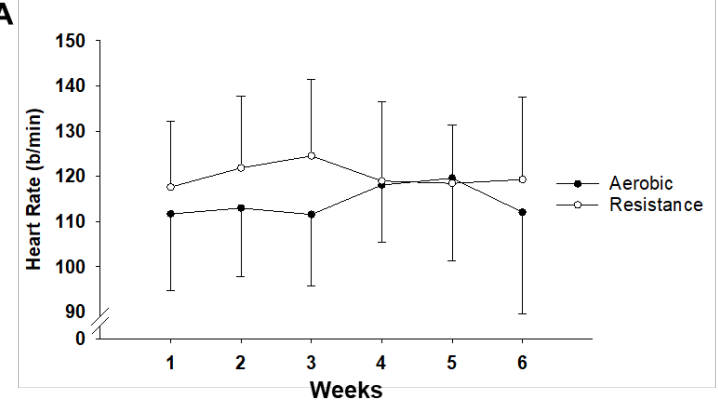

B

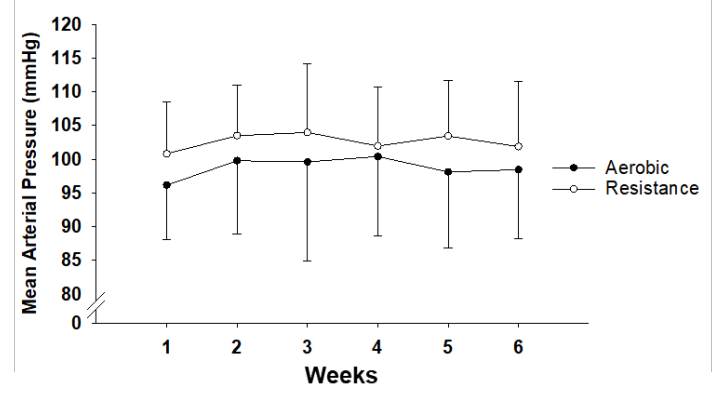

C

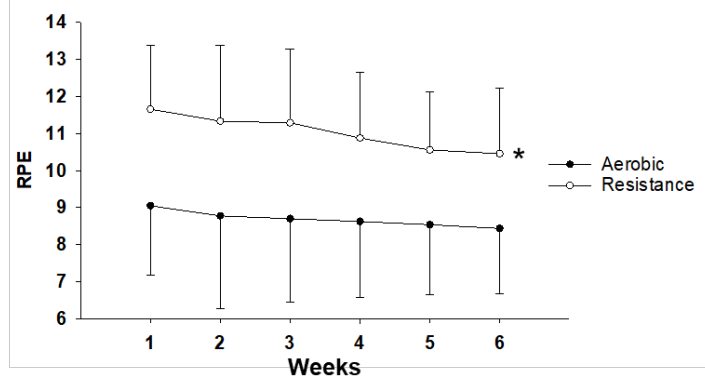

D

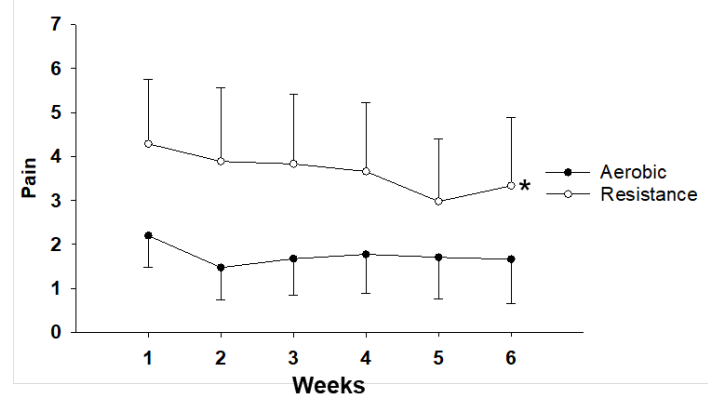

Figure 14: Mean weekly heart rate measured during exercise (A), mean arterial pressure measured during recovery (B), rating of perceived exertion (RPE) measured during exercise $(\mathrm{C})$, Pain measured during exercise and recovery (D). Variable different than aerobic $(\mathrm{P}<0.05)$ is indicated by *. Data are reported as mean $\pm \mathrm{SD}$. 

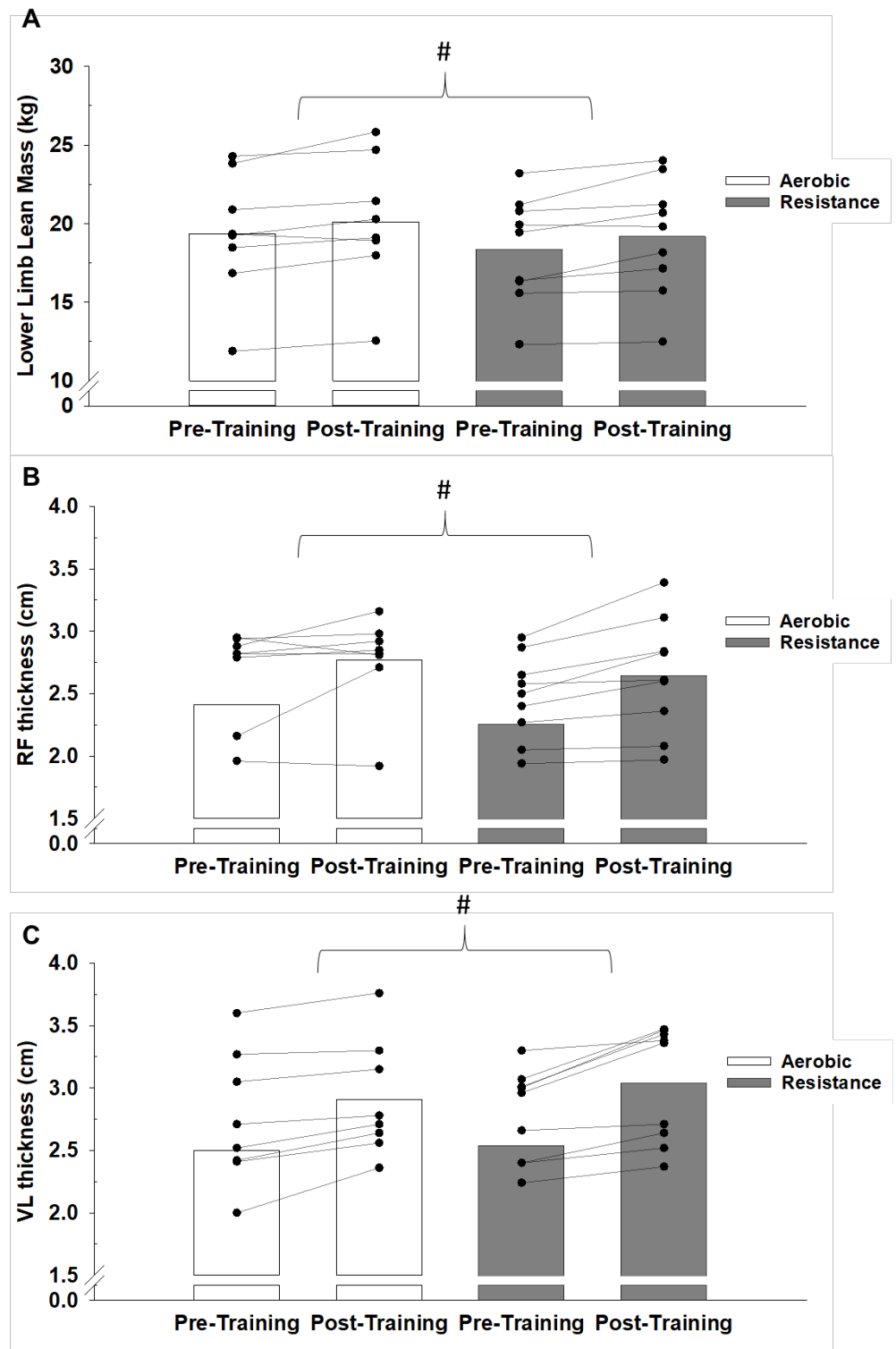

Figure 15: Pre- to post-training changes in lower limb lean mass (A), rectus femoris (RF) muscle thickness (B), and vastus lateralis (VL) muscle thickness (C). Significant main effect of training $(\mathrm{P}<0.05)$ is indicated by \#. Mean data represented as column, individual changes reported by black circles. 

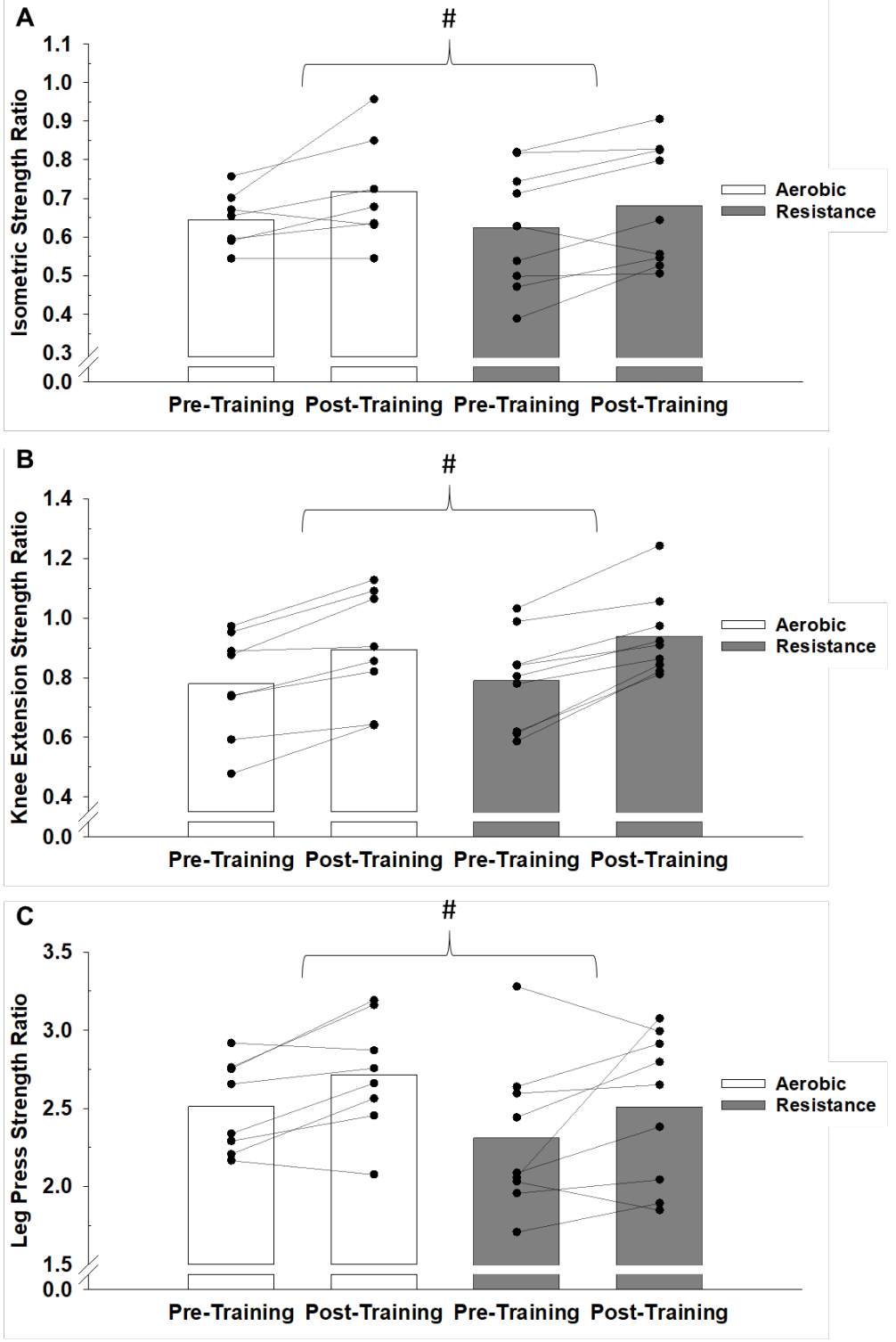

Figure 16: Pre- to post-training changes in isometric strength (A), Knee extension 1 repetition $\max (\mathrm{KE} 1 \mathrm{RM}$; B), and leg press 1 repetition max (LP 1 RM; C). Significant main effect of training $(\mathrm{P}<0.05)$ is indicated by \#. Mean data represented as column, individual changes reported by black circles. 


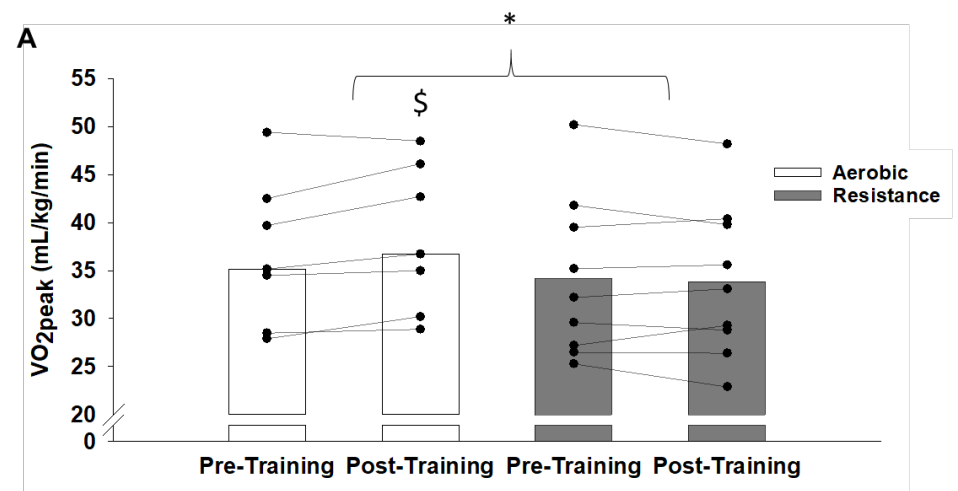

B

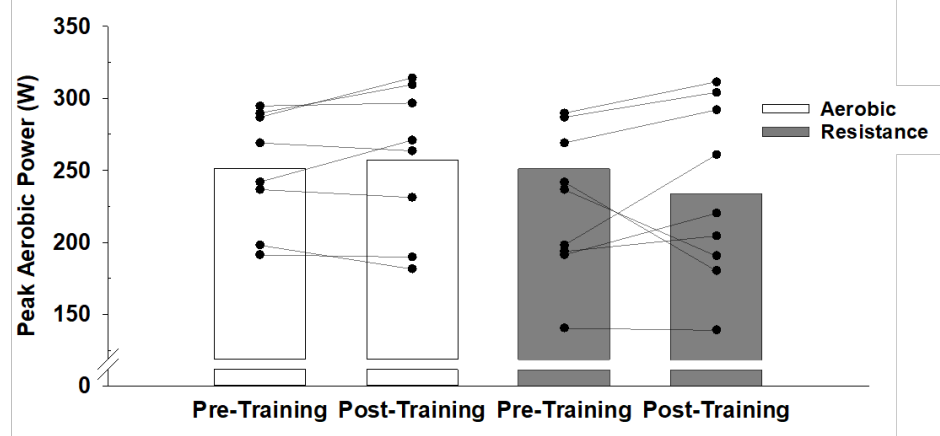

C

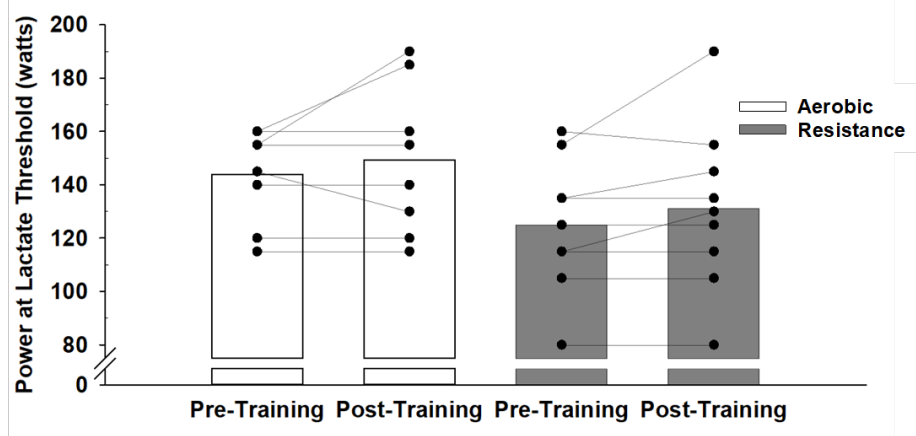

Figure 17: Pre- to post-training changes in $\mathrm{VO}_{2 \text { peak }}(\mathrm{A}), \mathrm{W}_{\text {peak }}(\mathrm{B})$, lactate threshold (C). Simple effect of training $(\mathrm{P}<0.05)$ is indicated by $\$$. Significant training $X$ group interaction $(\mathrm{P}<0.05)$ is indicated by *. Mean data represented as column, individual changes reported by black circles. 


\section{Conclusion}

Exercise with blood flow restriction (BFR) is emerging as an effective method to improve muscle size, strength, and exercise capacity healthy, clinical, and athletic populations (table 1). Exercise with BFR allows for the development of muscle size and strength at low training loads. This is especially important for clinical populations in which high training loads are contraindicated or impossible. Currently, there are no standardized guidelines for exercise with BFR. I used a variety of experimental techniques including ultrasound, near-infrared spectroscopy, expired air analysis, electrical stimulation, and dual-energy X-ray absorptiometry to investigate how cuff pressure and as well as the type of exercise alter acute and chronic responses to exercise with BFR.

In the first study, I was the first to report changes in blood flow, and tissue perfusion during aerobic exercise with BFR. Our key findings were the relative reduction in blood flow was generally maintained at rest. Additionally, as pressure increased, brachial artery blood flow and tissue perfusion decreased during and after exercise with BFR.

Ultimately, these results suggest cuff pressures between 60-80\% LOP, decrease blood flow and tissue perfusion without completely occluding blood flow, nor do they cause any acute cardiovascular changes, and therefore may be an adequate

In the second study, we examined the cardiovascular, metabolic, perceptual and neuromuscular effects of cycling with BFR. The major findings of this study were: 1) BFR caused a reduction in blood flow and tissue perfusion and augmented metabolite 
accumulation, 2) $\mathrm{VO}_{2}$, heart rate, ventilation, and RPE in the BFR 60 were lower than $\mathrm{HL}$ cycling, and 3) exercise with BFR impaired MVIC torque through peripheral and central mechanisms. These results suggest a cuff pressure of $\sim 60 \%$ limb occlusion pressure should be adequate for training as it results in reduce tissue perfusion, increased metabolite accumulation, and reduced neuromuscular function, without increasing cardiorespiratory, and perceptual responses to that of HL cycling.

Finally in the third study, I was the first to directly compare training adaptions to aerobic and resistance exercise with BFR. The main findings were that aerobic and resistance exercise with BFR similarly increased muscle size and strength, but only aerobic training increased exercise capacity. Additionally, aerobic exercise with BFR required less perceived effort and resulted in lower muscle pain. Collectively, these findings demonstrate clear advantages for performing aerobic training, and therefore practitioners considering implementing exercise training with BFR should use aerobic exercise (walking or cycling) if possible.

In conclusion, moderate cuff pressure of $\sim 60 \%$ limb occlusion pressure increases metabolic stress, without completely occluding blood flow, or causing excessive muscle pain. Moreover, this pressure results in significant increases in muscle size and strength following cycling and resistance training. Additionally, aerobic exercise with BFR may be the better method for training in populations who are unable to perform high-intensity exercise. Taken together, these studies will enable researchers, clinicians, and coaches to more effectively prescribe exercise with BFR to improve muscle size, strength and exercise capacity. 


\section{Reference List}

1. Clarke, T.C., et al., Early release of selected estimates based on data from the January-March 2015 National Health Interview Survey. National Center for Health Statistics, 2015. September 201(5).

2. Garber, C.E., et al., American College of Sports Medicine position stand. Quantity and quality of exercise for developing and maintaining cardiorespiratory, musculoskeletal, and neuromotor fitness in apparently healthy adults: guidance for prescribing exercise. Med Sci Sports Exerc, 2011. 43(7): p. 1334-59.

3. Manini, T.M. and B.C. Clark, Blood Flow Restricted Exercise and Skeletal Muscle Health. Exercise and Sport Sciences Reviews, 2009. 37(2): p. 78-85.

4. Ohta, H., et al., Low-load resistance muscular training with moderate restriction of blood flow after anterior cruciate ligament reconstruction. Acta Orthop Scand, 2003. 74(1): p. 62-8.

5. Yasuda, T., et al., Muscle size and arterial stiffness after blood flow-restricted low-intensity resistance training in older adults. Scandinavian journal of medicine \& science in sports, 2014. 24(5): p. 799-806.

6. Abe, T., C.F. Kearns, and Y. Sato, Muscle size and strength are increased following walk training with restricted venous blood flow from the leg muscle, Kaatsu-walk training. J Appl Physiol (1985), 2006. 100(5): p. 1460-6.

7. Fujita, T., et al., Increased muscle volume and strength following six days of lowintensity resistance training with restricted muscle blood flow. International Journal of KAATSU Training Research, 2008. 4(1): p. 1-8.

8. Kim, D., et al., The acute muscular effects of cycling with and without different degrees of blood flow restriction. Acta Physiol Hung, 2015. 102(4): p. 428-41.

9. Natsume, T., et al., Effects of Electrostimulation with Blood Flow Restriction on Muscle Size and Strength. Med Sci Sports Exerc, 2015. 47(12): p. 2621-7.

10. Abe, T., et al., Effects of low-intensity walk training with restricted leg blood flow on muscle strength and aerobic capacity in older adults. J Geriatr Phys Ther, 2010. 33(1): p. 34-40.

11. Bryk, F.F., et al., Exercises with partial vascular occlusion in patients with knee osteoarthritis: a randomized clinical trial. Knee Surg Sports Traumatol Arthrosc, 2016. 24(5): p. 1580-6. 
12. Gaunder, C.L., et al., Occlusion training: pilot study for postoperative lower extremity rehabilitation following primary total knee arthroplasty. US Army Med Dep J, 2017(2-17): p. 39-43.

13. Takarada, Y., H. Takazawa, and N. Ishii, Applications of vascular occlusion diminish disuse atrophy of knee extensor muscles. Med Sci Sports Exerc, 2000. 32(12): p. 2035-9.

14. Abe, T., et al., Eight days KAATSU-resistance training improved sprint but not jump performance in collegiate male track and field athletes. International Journal of KAATSU Training Research, 2005. 1(1): p. 19-23.

15. Takarada, Y., Y. Sato, and N. Ishii, Effects of resistance exercise combined with vascular occlusion on muscle function in athletes. Eur J Appl Physiol, 2002. 86(4): p. 308-14.

16. Luebbers, P.E., et al., The effects of a 7-week practical blood flow restriction program on well-trained collegiate athletes. The Journal of Strength \& Conditioning Research, 2014. 28(8): p. 2270-2280.

17. Scott, B.R., et al., Exercise with blood flow restriction: an updated evidencebased approach for enhanced muscular development. Sports Med, 2015. 45(3): p. 313-25.

18. Yasuda, T., et al., Muscle activation during low-intensity muscle contractions with restricted blood flow. J Sports Sci, 2009. 27(5): p. 479-89.

19. Scott, B.R., et al., Hypoxia and resistance exercise: a comparison of localized and systemic methods. Sports Med, 2014. 44(8): p. 1037-54.

20. Loenneke, J.P., et al., The anabolic benefits of venous blood flow restriction training may be induced by muscle cell swelling. Med Hypotheses, 2012. 78(1): p. 151-4.

21. Pierce, J.R., et al., Growth hormone and muscle function responses to skeletal muscle ischemia. J Appl Physiol (1985), 2006. 101(6): p. 1588-95.

22. Takarada, Y., et al., Rapid increase in plasma growth hormone after low-intensity resistance exercise with vascular occlusion. J Appl Physiol (1985), 2000. 88(1): p. 61-5.

23. Patterson, S.D. and C.R. Brandner, The role of blood flow restriction training for applied practitioners: A questionnaire-based survey. J Sports Sci, 2017: p. 1-8. 
24. Loenneke, J., R. Thiebaud, and T. Abe, Does blood flow restriction result in skeletal muscle damage? A critical review of available evidence. Scandinavian journal of medicine \& science in sports, 2014. 24(6): p. e415-422.

25. Abe T, K.C., Fujita S, Sakamaki M, Sato Y, Brechue WF, Skeletal muscle size and strength are increased following walk training with restricted leg muscle blood flow: implications for training duration and frequency. International Journal of KAATSU Training Research, 2009. 5(1): p. 9-15.

26. Loenneke, J.P., et al., Low intensity blood flow restriction training: a metaanalysis. Eur J Appl Physiol, 2012. 112(5): p. 1849-59.

27. Abe, T., et al., Exercise intensity and muscle hypertrophy in blood flow-restricted limbs and non-restricted muscles: a brief review. Clinical physiology and functional imaging, 2012. 32(4): p. 247-252.

28. Yasuda, T., et al., Effects of Low-Load, Elastic Band Resistance Training Combined With Blood Flow Restriction on Muscle Size and Arterial Stiffness in Older Adults. J Gerontol A Biol Sci Med Sci, 2015. 70(8): p. 950-8.

29. Loenneke, J.P., et al., Effects of exercise with and without different degrees of blood flow restriction on torque and muscle activation. Muscle \& nerve, 2015. 51(5): p. 713-721.

30. Suga, T., et al., Effect of multiple set on intramuscular metabolic stress during low-intensity resistance exercise with blood flow restriction. Eur J Appl Physiol, 2012. 112: p. 3915-3920.

31. Cook, S.B., B.C. Clark, and L.L. Ploutz-Snyder, Effects of exercise load and blood-flow restriction on skeletal muscle function. Med Sci Sports Exerc, 2007. 39(10): p. 1708-13.

32. Lixandrao, M.E., et al., Effects of exercise intensity and occlusion pressure after 12 weeks of resistance training with blood-flow restriction. Eur J Appl Physiol, 2015. 115(12): p. 2471-80.

33. Patterson, S.D. and R.A. Ferguson, Increase in calf post-occlusive blood flow and strength following short-term resistance exercise training with blood flow restriction in young women. European journal of applied physiology, 2010. 108(5): p. 1025-1033.

34. Corvino, R.B., et al., Physiological responses to interval endurance exercise at different levels of blood flow restriction. European journal of applied physiology, 2017. 117(1): p. 39-52. 
35. May, A.K., C.R. Brandner, and S.A. Warmington, Hemodynamic responses are reduced with aerobic compared with resistance blood flow restriction exercise. Physiol Rep, 2017. 5(3).

36. Abe, T., et al., Effects of Low-Intensity Cycle Training with Restricted Leg Blood Flow on Thigh Muscle Volume and VO2MAX in Young Men. J Sports Sci Med, 2010. 9(3): p. 452-8.

37. Corvino RB, O.M., Santos RPd, Denadai BS, Caputo F, Four weeks of blood flow restricted training increases time to exhaustion at severe intensity cycling exercise. . Rev. bras. cineantropom. desempenho hum. , 2014. 16(5): p. 570-578.

38. de Oliveira, M.F., et al., Short-term low-intensity blood flow restricted interval training improves both aerobic fitness and muscle strength. Scand J Med Sci Sports, 2016. 26(9): p. 1017-25.

39. Staunton, C.A., et al., Haemodynamics of aerobic and resistance blood flow restriction exercise in young and older adults. Eur J Appl Physiol, 2015. 115(11): p. 2293-302.

40. Loenneke, J., et al., Blood flow restriction: effects of cuff type on fatigue and perceptual responses to resistance exercise. Acta Physiologica Hungarica, 2014. 101(2): p. 158-166.

41. Loenneke, J.P., et al., Effect of cuff type on arterial occlusion. Clinical physiology and functional imaging, 2013.33(4): p. 325-327.

42. Rossow, L.M., et al., Cardiovascular and perceptual responses to blood-flowrestricted resistance exercise with differing restrictive cuffs. Clinical physiology and functional imaging, 2012. 32(5): p. 331-337.

43. Loenneke, J.P., et al., Effects of cuff width on arterial occlusion: implications for blood flow restricted exercise. European journal of applied physiology, 2012. 112(8): p. 2903-2912.

44. Mouser, J.G., et al., A tale of three cuffs: the hemodynamics of blood flow restriction. Eur J Appl Physiol, 2017. 117(7): p. 1493-1499.

45. Laurentino, G.C., et al., The effect of cuff width on muscle adaptations after blood flow restriction training. Medicine \& Science in Sports \& Exercise, 2016. 48(5): p. 920-925.

46. Fahs, C.A., et al., Methodological considerations for blood flow restricted resistance exercise. Journal of Trainology, 2012. 1(1): p. 14-22. 
47. Mattocks, K.T., et al., The effects of upper body exercise across different levels of blood flow restriction on arterial occlusion pressure and perceptual responses. Physiol Behav, 2017. 171: p. 181-186.

48. Counts, B.R., et al., Influence of relative blood flow restriction pressure on muscle activation and muscle adaptation. Muscle \& nerve, 2016. 53(3): p. 438445.

49. Mouser, J.G., et al., Brachial blood flow under relative levels of blood flow restriction is decreased in a nonlinear fashion. Clin Physiol Funct Imaging, 2017.

50. Hughes, L., et al., Influence and reliability of lower-limb arterial occlusion pressure at different body positions. PeerJ, 2018. 6: p. e4697.

51. Sieljacks, P., et al., Body position influences arterial occlusion pressure: implications for the standardization of pressure during blood flow restricted exercise. European journal of applied physiology, 2018. 118(2): p. 303-312.

52. Wilson, J.M., et al., Practical blood flow restriction training increases acute determinants of hypertrophy without increasing indices of muscle damage. The Journal of Strength \& Conditioning Research, 2013. 27(11): p. 3068-3075.

53. Younger, A.S., J.A. McEwen, and K. Inkpen, Wide contoured thigh cuffs and automated limb occlusion measurement allow lower tourniquet pressures. Clinical orthopaedics and related research, 2004. 428: p. 286-293.

54. Hunt, J.E., C. Stodart, and R.A. Ferguson, The influence of participant characteristics on the relationship between cuff pressure and level of blood flow restriction. European journal of applied physiology, 2016: p. 1-12.

55. Crenshaw, A.G., et al., Wide tourniquet cuffs more effective at lower inflation pressures. Acta Orthopaedica Scandinavica, 1988. 59(4): p. 447-451.

56. Loenneke, J.P., et al., Blood flow restriction in the upper and lower limbs is predicted by limb circumference and systolic blood pressure. European journal of applied physiology, 2015. 115(2): p. 397-405.

57. Fitschen, P., et al., Perceptual effects and efficacy of intermittent or continuous blood flow restriction resistance training. Clinical physiology and functional imaging, 2014. 34(5): p. 356-363.

58. Yasuda, T., et al., Influence of continuous or intermittent blood flow restriction on muscle activation during low-intensity multiple sets of resistance exercise. Acta Physiologica Hungarica, 2013. 100(4): p. 419-426. 
59. Nakajima, T., et al., Use and safety of KAATSU training: results of a national survey. International Journal of KAATSU Training Research, 2006. 2(1): p. 5-13.

60. Loenneke, J.P., et al., Blood flow restriction does not result in prolonged decrements in torque. European journal of applied physiology, 2013. 113(4): p. 923-931.

61. Cayot, T.E., et al., Effects of blood flow restriction duration on muscle activation and microvascular oxygenation during low-volume isometric exercise. Clinical physiology and functional imaging, 2016. 36(4): p. 298-305.

62. Takada, S., et al., Low-intensity exercise can increase muscle mass and strength proportionally to enhanced metabolic stress under ischemic conditions. Journal of applied physiology, 2012. 113(2): p. 199-205.

63. H. Iida, et al., Hemodynamic and autonomic nervous responses to the restriction of femoral blood flow by KAATSU. Int. J. KAATSU Training Res, 2005. 1: p. 5764.

64. Lauver, J.D., et al., The effect of eccentric exercise with blood flow restriction on neuromuscular activation, microvascular oxygenation, and the repeated bout effect. European journal of applied physiology, 2017. 117(5): p. 1005-1015.

65. Yanagisawa, O. and M. Sanomura, Effects of low-load resistance exercise with blood flow restriction on high-energy phosphate metabolism and oxygenation level in skeletal muscle. Interventional Medicine and Applied Science, 2017. 9(2): p. 67-75.

66. Nyberg, S.K., et al., Reliability of forearm oxygen uptake during handgrip exercise: assessment by ultrasonography and venous blood gas. Physiological reports, 2018. 6(10): p. e13696.

67. Bezerra de Morais, A.T., et al., Upper limbs total occlusion pressure assessment: D oppler ultrasound reproducibility and determination of predictive variables. Clinical physiology and functional imaging, 2017. 37(4): p. 437-441.

68. Celie, B., et al., Reliability of near infrared spectroscopy (NIRS) for measuring forearm oxygenation during incremental handgrip exercise. European journal of applied physiology, 2012. 112(6): p. 2369-2374.

69. Davis, J.F., Manual of surface electromyography. 1959, Allan Memorial Inst of Psychiatry Montreal (Quebec).

70. Cohen, J., Statistical power analysis for the behavioral sciences. Vol. 2nd edition. 1988, NJ: Hilsdale. 
71. Ganesan, G., et al., Effect of blood flow restriction on tissue oxygenation during knee extension. Med Sci Sports Exerc, 2015. 47(1): p. 185-93.

72. Gobbo, M., et al., Transcutaneous neuromuscular electrical stimulation: influence of electrode positioning and stimulus amplitude settings on muscle response. European journal of applied physiology, 2011. 111(10): p. 2451-2459.

73. Downs, M.E., et al., Acute vascular and cardiovascular responses to blood flowrestricted exercise. Med Sci Sports Exerc, 2014. 46(8): p. 1489-97.

74. Takano, H., et al., Hemodynamic and hormonal responses to a short-term lowintensity resistance exercise with the reduction of muscle blood flow. Eur J Appl Physiol, 2005. 95(1): p. 65-73.

75. Pyke, K.E., V. Poitras, and M.E. Tschakovsky, Brachial artery flow-mediated dilation during handgrip exercise: evidence for endothelial transduction of the mean shear stimulus. Am J Physiol Heart Circ Physiol, 2008. 294(6): p. H266979.

76. Taylor, W.F., et al., Cutaneous vascular responses to isometric handgrip exercise. J Appl Physiol (1985), 1989. 66(4): p. 1586-92.

77. Kamiya, A., et al., Static handgrip exercise modifies arterial baroreflex control of vascular sympathetic outflow in humans. Am J Physiol Regul Integr Comp Physiol, 2001. 281(4): p. R1134-9.

78. Shoemaker, J.K., M.J. MacDonald, and R.L. Hughson, Time course of brachial artery diameter responses to rhythmic handgrip exercise in humans. Cardiovasc Res, 1997. 35(1): p. 125-31.

79. Green, D.J., et al., Comparison of forearm blood flow responses to incremental handgrip and cycle ergometer exercise: relative contribution of nitric oxide. $\mathrm{J}$ Physiol, 2005. 562(Pt 2): p. 617-28.

80. Boushel, R., et al., Regional blood flow during exercise in humans measured by near-infrared spectroscopy and indocyanine green. J Appl Physiol (1985), 2000. 89(5): p. 1868-78.

81. Habazettl, H., et al., Near-infrared spectroscopy and indocyanine green derived blood flow index for noninvasive measurement of muscle perfusion during exercise. J Appl Physiol (1985), 2010. 108(4): p. 962-7.

82. Spranger, M.D., et al., Blood flow restriction training and the exercise pressor reflex: a call for concern. American Journal of Physiology-Heart and Circulatory Physiology, 2015. 309(9): p. H1440-H1452. 
83. Shoemaker, J.K., et al., WISE 2005: stroke volume changes contribute to the pressor response during ischemic handgrip exercise in women. J Appl Physiol (1985), 2007. 103(1): p. 228-33.

84. Kaufman, M.P. and S.G. Hayes, The exercise pressor reflex. Clin Auton Res, 2002. 12(6): p. 429-39.

85. Nakajima, T., et al., Use and safety of KAATSU training: results of a national survey. . International Journal of KAATSU Training Research, 2006. 2(1): p. 513.

86. Park, S., et al., Increase in maximal oxygen uptake following 2-week walk training with blood flow occlusion in athletes. Eur J Appl Physiol, 2010. 109(4): p. 591600 .

87. Hackney, K.J., et al., Blood flow-restricted exercise in space. Extrem Physiol Med, 2012. 1(1): p. 12.

88. Manini, T.M. and B.C. Clark, Blood flow restricted exercise and skeletal muscle health. Exerc Sport Sci Rev, 2009. 37(2): p. 78-85.

89. Bigland-Ritchie, B. and J. Woods, Changes in muscle contractile properties and neural control during human muscular fatigue. Muscle \& Nerve: Official Journal of the American Association of Electrodiagnostic Medicine, 1984. 7(9): p. 691699.

90. Hakkinen, K. and A. Pakarinen, Acute hormonal responses to two different fatiguing heavy-resistance protocols in male athletes. J Appl Physiol (1985), 1993. 74(2): p. 882-7.

91. Cook, S.B., J.A. Kanaley, and L.L. Ploutz-Snyder, Neuromuscular function following muscular unloading and blood flow restricted exercise. Eur J Appl Physiol, 2014. 114(7): p. 1357-65.

92. Karabulut, M., et al., Neuromuscular fatigue following low-intensity dynamic exercise with externally applied vascular restriction. Journal of Electromyography and Kinesiology, 2010. 20(3): p. 440-447.

93. Husmann, F., et al., Impact of Blood Flow Restriction Exercise on Muscle Fatigue Development and Recovery. Medicine and science in sports and exercise, 2018. 50(3): p. 436-446.

94. Ogawa, M., et al., Time course changes in muscle size and fatigue during walking with restricted leg blood flow in young men. Journal of Physical Education and Sports Management, 2012. 3(1): p. 14-19. 
95. Bigland-Ritchie, B., et al. Task-dependent factors in fatigue of human voluntary contractions. in Fatigue. 1995. Springer.

96. Garber, C.E., et al., Quantity and quality of exercise for developing and maintaining cardiorespiratory, musculoskeletal, and neuromotor fitness in apparently healthy adults: guidance for prescribing exercise. Medicine \& Science in Sports \& Exercise, 2011. 43(7): p. 1334-1359.

97. Lucia, A., et al., Analysis of the aerobic-anaerobic transition in elite cyclists during incremental exercise with the use of electromyography. Br J Sports Med, 1999. 33(3): p. 178-85.

98. Wray, D.W., et al., Progressive handgrip exercise: evidence of nitric oxidedependent vasodilation and blood flow regulation in humans. American Journal of Physiology-Heart and Circulatory Physiology, 2011. 300(3): p. H1101-H1107.

99. Lucero, A.A., et al., Reliability of muscle blood flow and oxygen consumption response from exercise using near-infrared spectroscopy. Experimental physiology, 2018. 103(1): p. 90-100.

100. Borg, G.A., Psychophysical bases of perceived exertion. Med Sci Sports Exerc, 1982. 14(5): p. 377-81.

101. DeLoach, L.J., et al., The visual analog scale in the immediate postoperative period: intrasubject variability and correlation with a numeric scale. Anesthesia \& Analgesia, 1998. 86(1): p. 102-106.

102. Pietrosimone, B.G., et al., Electrode type and placement configuration for quadriceps activation evaluation. 2011, National Athletic Trainers' Association, Inc.

103. Gandevia, S.C., Spinal and supraspinal factors in human muscle fatigue. Physiological reviews, 2001. 81(4): p. 1725-1789.

104. Aagaard, P., et al., Increased rate of force development and neural drive of human skeletal muscle following resistance training. Journal of applied physiology, 2002. 93(4): p. 1318-1326.

105. Cohen, J., Statistical power analysis for the behavioral sciences. 2nd. 1988, Hillsdale, NJ: erlbaum.

106. Takano, H., et al., Hemodynamic and hormonal responses to a short-term lowintensity resistance exercise with the reduction of muscle blood flow. European journal of applied physiology, 2005. 95(1): p. 65-73. 
107. Schrage, W.G., M.J. Joyner, and F.A. Dinenno, Local inhibition of nitric oxide and prostaglandins independently reduces forearm exercise hyperaemia in humans. The Journal of physiology, 2004. 557(2): p. 599-611.

108. Loenneke, J.P., et al., The energy requirement of walking with restricted blood flow. Sport Science, 2011. 4(2): p. 7-11.

109. Mendonca, G.V., et al., Metabolic cost of locomotion during treadmill walking with blood flow restriction. Clinical physiology and functional imaging, 2014. 34(4): p. 308-316.

110. Mattocks, K.T., et al., The effects of upper body exercise across different levels of blood flow restriction on arterial occlusion pressure and perceptual responses. Physiology \& behavior, 2017. 171: p. 181-186.

111. Jankowski, M.P., et al., Comprehensive phenotyping of group III and IV muscle afferents in mouse. Journal of neurophysiology, 2013. 109(9): p. 2374-2381.

112. Haouzi, P., et al., Responses of group III and IV muscle afferents to distension of the peripheral vascular bed. Journal of Applied Physiology, 1999. 87(2): p. 545553.

113. Graven-Nielsen, T., et al., Inhibition of maximal voluntary contraction force by experimental muscle pain: a centrally mediated mechanism. Muscle \& Nerve: Official Journal of the American Association of Electrodiagnostic Medicine, 2002. 26(5): p. 708-712.

114. Mouser, J.G., et al., Blood flow restriction and cuff width: effect on blood flow in the legs. Clinical physiology and functional imaging, 2018.

115. Slysz, J., J. Stultz, and J.F. Burr, The efficacy of blood flow restricted exercise: A systematic review \& meta-analysis. Journal of science and medicine in sport, 2016. 19(8): p. 669-675.

116. Hughes, L., et al., Blood flow restriction training in clinical musculoskeletal rehabilitation: a systematic review and meta-analysis. Br J Sports Med, 2017. 51(13): p. 1003-1011.

117. Scott, B.R., et al., Blood flow restricted exercise for athletes: A review of available evidence. Journal of science and medicine in sport, 2016. 19(5): p. 360367.

118. Evans, C., S. Vance, and M. Brown, Short-term resistance training with blood flow restriction enhances microvascular filtration capacity of human calf muscles. Journal of sports sciences, 2010. 28(9): p. 999-1007. 
119. Larkin, K.A., et al., Blood flow restriction enhances post-resistance exercise angiogenic gene expression. Med Sci Sports Exerc, 2012. 44(11): p. 2077-83.

120. Medicine, A.C.o.S., ACSM's guidelines for exercise testing and prescription. 2013: Lippincott Williams \& Wilkins.

121. Twist, C. and R. Eston, The effects of exercise-induced muscle damage on maximal intensity intermittent exercise performance. Eur J Appl Physiol, 2005. 94(5-6): p. 652-8.

122. Pritchard, J., et al., Evaluation of dual energy X-ray absorptiometry as a method of measurement of body fat. European journal of clinical nutrition, 1993. 47(3): p. 216-228.

123. Chilibeck, P., et al., Reproducibility of dual-energy $x$-ray absorptiometry. Canadian Association of Radiologists journal= Journal l'Association canadienne des radiologistes, 1994. 45(4): p. 297-302.

124. Brzycki, M., Strength testing: predicting one-rep max from repetitions to fatigue. . Journal of Physical Education, Recreation \& Dance, 1993. 64(1): p. 88-90.

125. Abdul-Hameed, U., et al., Reliability of 1-repetition maximum estimation for upper and lower body muscular strength measurement in untrained middle aged type 2 diabetic patients. Asian journal of sports medicine, 2012. 3(4): p. 267.

126. Foster, C., et al., A new approach to monitoring exercise training. The Journal of Strength \& Conditioning Research, 2001. 15(1): p. 109-115.

127. Newell, J., et al., Software for calculating blood lactate endurance markers. Journal of sports sciences, 2007. 25(12): p. 1403-1409.

128. Cheng, B., et al., A new approach for the determination of ventilatory and lactate thresholds. International journal of sports medicine, 1992. 13(07): p. 518-522.

129. Abe, T., et al., Skeletal muscle size and circulating IGF-1 are increased after two weeks of twice daily "KAATSU” resistance training. International Journal of KAATSU Training Research, 2005. 1(1): p. 6-12.

130. Yasuda, T., et al., Muscle fiber cross-sectional area is increased after two weeks of twice daily KAATSU-resistance training. International Journal of KAATSU Training Research, 2005. 1(2): p. 65-70.

131. Loenneke, J., et al., Blood flow restriction pressure recommendations: the hormesis hypothesis. Medical hypotheses, 2014. 82(5): p. 623-626. 
132. Hill, D.W., et al., Effect of sampling strategy on measures of VO2peak obtained using commercial breath-by-breath systems. European journal of applied physiology, 2003. 89(6): p. 564-569.

133. Skinner, J.S., et al., Reproducibility of maximal exercise test data in the HERITAGE family study. Medicine and science in sports and exercise, 1999. 31(11): p. 1623-1628.

134. Esbjörnsson, M., et al., Muscle fibre types and enzyme activities after training with local leg ischaemia in man. Acta physiologica scandinavica, 1993. 148(3): p. 233-242.

135. Sundberg, C.J., Exercise and training during graded leg ischaemia in healthy man with special reference to effects on skeletal muscle. Acta physiologica Scandinavica. Supplementum, 1994. 615: p. 1-50. 


\section{A Approved IRB forms}

\section{A.1 Study 1}

\section{Michigan Technological}

DATE:

TO:

FROM:

RE:

TITLE:

SUBMISSION TYPE:

STATUS:
February 20, 2017

Steven Elmer, PhD, KIP

Joanne Polzien, Executive Director

M1581, [1024563-1]

Effects of Occlusion Cuff Pressure and Size on Blood Flow During Exercise with Blood Flow Restriction

New Project

New Project, APPROVED

The Institutional Review Board (IRB) has reviewed and approved your research application as compliant with all applicable sections of the federal regulations, Michigan law, and Michigan Tech IRB policies and procedures. All research must be conducted in accordance with this approved submission.

APPROVAL DATE: February 17, 2017

EXPIRATION DATE: February 16, 2018

A pdf of this signed memo and any stamped approved documents, if applicable, have been placed in the review details under "board documents" for this project.

This approval applies only for this project, and only under the conditions and procedures described in the application. If/when changes become necessary but are not limited to: changes in protocol, personnel, study location, participant recruitment, etc., as set forth in this approval, you must follow the INSTRUCTIONS and submit the FORM for Change Request during approval found in the IRBNet Library. You must receive notification of approval PRIOR to implementing the change(s).

Approvals are granted for up to a one year period. You are responsible for submitting requests for continuation in advance of the expiration date for each year of the project. You will need to request a continuation six weeks prior to the end date indicated above. It is very important that the expiration date is not missed. Failure to submit annual review materials on time will result in the termination of this protocol on the expiration date listed above.

Please note the following in order to comply with federal regulations and IRB policy:

1. Please remember that informed consent is a process beginning with a description of the study and assurance of participant understanding followed by a signed consent form. Informed consent must continue throughout the study via a dialogue between the researcher and research participant. Federal regulations require each participant receive a copy of the signed consent document. 
2. Individual identification of human subjects in any publication is an invasion of privacy. Before beginning a project involving human subjects, and only if required, the principal investigator must obtain a properly executed informed consent from each subject and/or the person legally responsible for the subject. If a consent form has been reviewed and approved it has been attached with an official date stamp on it. Only copies of the official date stamped informed consent are to be distributed to participants relating to this project. If any changes or modifications are needed regarding this form, you must first submit the revised document for review and approval prior to use. The PI must retain informed consent forms on file for at least three years after the end of the project.

3. The approved project will be subject to periodic review. This review will consist of consulting with the $\mathrm{PI}$ and examining the appropriate project records. All required research records must be securely retained in either paper or electronic format for a minimum of three years following the closure of the approved study. This includes signed consent documents from all participants.

4. All Unanticipated Problems / Serious Adverse Events to participants or other parties affected by the research must be reported to this office within two days of the event occurrence. All instances of non-compliance or complaints regarding this study must be reported to this office in a timely manner. Please use the INSTRUCTIONS and FORM: Unanticipated Problem / Serious Adverse Event Form found both on our web site and the IRBNet Library.

If you have any questions, please contact the Compliance, Integrity, and Safety Office at 906.487 .2902 or send your message via email through IRBNet using the Send Project Mail feature. 


\title{
CONSENT FORM
}

\section{Exercise with Blood Flow Restriction}

\author{
Matthew A Kilgas and Steven J. Elmer Department \\ of Kinesiology \& Integrative Physiology Michigan \\ Technological University \\ Houghton, MI 49931 \\ Phone: (906) 487-2324 \\ Fax: (906) 487-0985 \\ Email: makilgas@mtu.edu and sielemer@mtu.edu
}

\section{Introduction}

You are invited to participate in a research study being conducted by Dr. Steven Elmer, a faculty member in the Department of Kinesiology and Integrative Physiology at Michigan Technological University. The purpose of this study is to establish better training guidelines for a new type of exercise called "exercise with blood flow restriction". This type of exercise consists of performing light weight lifting movements ( $20 \%$ of your maximum) while using an inflatable cuff (similar to a tourniquet) to restrict the blood coming back from the muscles to the heart. This type of exercise provides a hard workout for muscles without over taxing the heart and lungs.

You will be excluded from this study if you:

- Have any physical limitations that prevent you from performing arm and leg weight lifting exercises

- Are a smoker

- Have diabetes

- Have any cardiopulmonary disorders, including but not limited to hypertension

\section{Experimental Protocol and Brief Risk Summary}

Human Performance Laboratory (Student Development Complex Room P105):

Orientation Visit: We will ask you to attend a required orientation session prior to participating in this research study. During this visit, the procedures and equipment will be explained to you, and you will be able to ask questions about what you will do. For this study we will ask you to participate in six total visits ( 2 familiarization visit and 4 exercise sessions).

\section{Forearm and Leg Familiarization Visits}

Forearm and leg blood flow: You will be positioned comfortably on an examination table, and a small roundshaped ultrasound probe will placed on your skin by your elbow for the forearm test, and on the front of the hip for the leg blood flow measurement. This device will enable us to measure the blood flow to your arms and legs/. Ultrasound machines are used in clinical settings and there is minimal risk involved. You may experience some redness on your skin resulting from the contact of the ultrasound probe and gel. However, this is only temporary and should resolve quickly. Although the risk of an allergic reaction (itching, redness, small raised bumps, and/or burning, on and near the area of contact) is rare, the risk is possible. Symptoms are generally short-lived (few days to few weeks) and you should seek immediate medical attention in case of a severe reaction.

Grip Strength: You will be asked to squeeze a handheld device as hard as possible for $3-5$ seconds. This test does not pose any risks.

\begin{tabular}{c|r|r|}
\multirow{3}{*}{$\begin{array}{c}\text { Michigan Tech } \\
\text { IRB APPROVAL }\end{array}$} & Approved on: & $02-17-2017$ \\
\cline { 2 - 3 } & Expires on: & $02-16-2018$ \\
\cline { 2 - 3 } & Study number: & $\begin{array}{l}1024563-1 . \\
\text { M1581 }\end{array}$ \\
\cline { 2 - 3 } & &
\end{tabular}


Leg Muscle strength: You will be instructed to perform brief max effort muscle contractions with your knee muscles on a device that is similar to standard weight lifting equipment. Prior to conducting these contractions you will warm-up with 10 minutes of low intensity cycling at a self-selected pace. All of these muscle contractions are short (5 seconds) and you will likely experience only minimal discomfort.

Occlusion pressure: An inflatable cuff will be placed around your arm or leg. The researcher will inflate the cuff until blood flow is no longer detected. The research will then immediately release the pressure in the cuff. This procedure is similar to having blood pressure taken. This test does not have any associated risks, however the cuff may be uncomfortable, but it will only last a few seconds.

\section{Exercise Sessions}

You will be asked to complete four exercise sessions (two for the arms and two for the legs) in which a different size occlusion cuffs will be use to restrict blood flow into your arms and legs. The pressure in the occlusion cuff will be randomly set to $0 \%, 50 \%$, and $130 \%$ of the occlusion pressure at rest. The researcher will measure blood flow for one minute prior to exercise, during exercise and for 1 minute flowing exercise. You will be asked to perform 9 sets of 30 repetitions with 10 minutes rest between sets. The load will vary, but will be fairly light.

Blood flow restricted exercise: this exercise is widely used in Japan and is now emerging in the United States. This form of training, which involves lifting light weights while partially occluding blow flow returning from the working muscles, has been successfully utilized with healthy active individuals, individuals recovering from ACL injury, and older adults. ${ }^{9}$ In a national Japanese survey of facilities using blood flow restricted exercise in which over 13,000 individuals engaged in training various modes of blood flow restricted exercise (walking, cycling, weight training), the most common side effects were bruising at the site of the tourniquet cuff (incidence $=13.1 \%$ ) and temporary numbness $(1.3 \%)$. More serious side effects were rare and included: venous thrombosis $(0.055 \%)$, cerebral infarction $(0.008 \%)$, rhabdomyolysis $(0.008 \%)$ and pulmonary embolism $(0.008 \%)$. Although these events are extremely rare, it should be noted that determining the relative safety of blood flow restriction requires a detailed study of several potential outcomes, and we acknowledge that unforeseen risks do exist. To minimize these risks even further we will limit the training to approximately 15 minutes and the pressure in the cuff will be released between exercises.

Electrical activity: Electromyography is method to measure the electrical activity of the muscles. The researcher will place small sensors over your arm and leg muscles. For these procedures, slight skin irritation may occur from the small sensors placed on the muscles. Although the risk of an allergic reaction (itching, redness, small raised bumps, and/or burning, on and near the area of contact) is rare, the risk is possible. Symptoms are generally short-lived (few days to few weeks) and you should seek immediate medical attention in case of a severe reaction.

Oxygen content: A NIRS system will be used to detect changes in oxygen in your blood. This is a non-invasive measurement simply obtained by sending near infrared light into the tissue and recording the amount of light that is transmitted through the tissue with a receiving electrode. A NIRS electrode will be placed on the thigh or forearm and secured to the skin with double sided tape. An ace bandage will be used to help secure the electrode and minimize movement on the skin. NIRS does not pose any risk other than perhaps some discomfort of having the device attached to your arm or leg for the duration of the experiment.

\section{BENEFITS}

We cannot promise any direct benefit for taking part in this study. You will, however, be provided with results from strength and performance testing. In addition, the results may help researchers and clinicians develop better

\begin{tabular}{c|r|l|}
\multicolumn{1}{c}{ Michigan Tech } & Approved on: & \multicolumn{1}{c|}{$02-17-2017$} \\
\cline { 2 - 3 } IRB APPROVAL & Expires on: & $02-16-2018$ \\
\cline { 2 - 3 } & Study number: & $\begin{array}{l}1024563-1, \\
\text { M1581 }\end{array}$ \\
\cline { 2 - 3 } & & \multicolumn{2}{|c}{}
\end{tabular}


exercise training programs.

\section{COMPENSATION}

You will not be compensated for your participation in this study.

\section{COST OF PARTICIPATION}

There will be no cost to you for participating in this research.

\section{RESEARCH RELATED INJURY}

In the event of physical and/or mental injury resulting from participation in this study, Michigan Technological University does not provide any medical, hospitalization or other insurance for participants in this study, nor will Michigan Technological University provide any medical treatment or compensation for any injury sustained as a result of participation in this study, except as required by law. If you are taking medications, it is your responsibility to consult with your physician regarding your participation in this study. Do not volunteer for this study if you have been instructed to abstain from this study by a physician. Any problems you experience throughout this study should be discussed immediately with your physician.

\section{CONFIDENTIALY OF RECORDS}

We will treat your identity with professional standards of confidentiality. The data from this study may be published, but your identity will not be shown. Paper files with your information and corresponding six digit code will be stored in a locked file cabinet in Dr. Elmer's office (Student Development Complex P105A) for three years after this study is completed. The Michigan Technological University Institutional Review Board (IRB) reserves the right to inspect both the research data collected and your experimental records.

\section{WITHDRAWAL}

Your participation is voluntary. Dr. Steven Elmer or Mr. Matthew Kilgas will answer any questions you have about the study. Any significant findings which develop during the course of the study which in our opinion may affect your desire to participate will be provided to you as soon as possible. Again, we will ask you to complete 8 weeks of testing but please note that you are free to withdraw your consent and stop participating at any time and for any reason. This includes the right to withdraw during the actual test. If you withdraw yourself during the actual testing, you will still be compensated for that session.

\section{PARTICIPANT'S RIGHTS INFORMATION}

The Michigan Tech Institutional Review Board has reviewed my request to conduct this project. If you have any concerns about your rights in this study, please contact the Office of Compliance, Integrity, and Safety at 906487-2902 or email $\underline{\text { RBB@mtu.edu. }}$

1, , have read through this consent form. The investigator provided me an opportunity to ask questions and I wish to voluntarily participate in this study.

Signature of Participant

Signature of Investigator

Michigan Tech

IRB APPROVAL

\begin{tabular}{|r|l|}
\hline Approved on: & $02-17-2017$ \\
\hline Expires on: & $02-16-2018$ \\
\hline Study number: & $\begin{array}{l}1024563-1 \\
M 1581\end{array}$ \\
\hline
\end{tabular}

Date

Date 


\section{A.2 Study 2}

\section{Michigan \\ 11. Technological \\ 1885 University}

Compliance, Integrity, and Safety

302 Lakeshore Center | 1400 Townsend Drive Houghton, MI 49931-1295 906-487-2902 | f. 906-487-2245
DATE:

TO:

FROM:

RE:

TITLE:
July 31,2017

Steven Elmer, PhD, KIP

Joanne Polzien, Executive Directo

M1625, [1082133-2]

The Acute Effects of Cycling with Blood Flow Restriction on Femoral Blood Flow and Muscular Fatigue

SUBMISSION TYPE:

Response/Follow-Up

STATUS:
New Project, APPROVED

The Institutional Review Board (IRB) has reviewed and approved your research application as compliant with all applicable sections of the federal regulations, Michigan law, and Michigan Tech IRB policies and procedures. All research must be conducted in accordance with this approved submission.

APPROVAL DATE: July 31, 2017

EXPIRATION DATE: July 30, 2018

A pdf of this signed memo and any stamped approved documents, if applicable, have been placed in the review details under "board documents" for this project.

This approval applies only for this project, and only under the conditions and procedures described in the application. If/when changes become necessary but are not limited to: changes in protocol, personnel, study location, participant recruitment, etc., as set forth in this approval, you must follow the INSTRUCTIONS and submit the FORM for Change Request during approval found in the IRBNet Library. You must receive notification of approval PRIOR to implementing the change(s).

Approvals are granted for up to a one year period. You are responsible for submitting requests for continuation in advance of the expiration date for each year of the project. You will need to request a continuation six weeks prior to the end date indicated above. It is very important that the expiration date is not missed. Failure to submit annual review materials on time will result in the termination of this protocol on the expiration date listed above.

Please note the following in order to comply with federal regulations and IRB policy:

1. Please remember that informed consent is a process beginning with a description of the study and assurance of participant understanding followed by a signed consent form. Informed consent must continue throughout the study via a dialogue between the researcher and research participant. Federal regulations require each participant receive a copy of the signed consent document. 
2. Individual identification of human subjects in any publication is an invasion of privacy. Before beginning a project involving human subjects, and only if required, the principal investigator must obtain a properly executed informed consent from each subject and/or the person legally responsible for the subject. If a consent form has been reviewed and approved it has been attached with an official date stamp on it. Only copies of the official date stamped informed consent are to be distributed to participants relating to this project. If any changes or modifications are needed regarding this form, you must first submit the revised document for review and approval prior to use. The PI must retain informed consent forms on file for at least three years after the end of the project.

3. The approved project will be subject to periodic review. This review will consist of consulting with the $\mathrm{PI}$ and examining the appropriate project records. All required research records must be securely retained in either paper or electronic format for a minimum of three years following the closure of the approved study. This includes signed consent documents from all participants.

4. All Unanticipated Problems / Serious Adverse Events to participants or other parties affected by the research must be reported to this office within two days of the event occurrence. All instances of non-compliance or complaints regarding this study must be reported to this office in a timely manner. Please use the INSTRUCTIONS and FORM: Unanticipated Problem / Serious Adverse Event Form found both on our web site and the IRBNet Library.

If you have any questions, please contact the Compliance, Integrity, and Safety Office at 906.487 .2902 or send your message via email through IRBNet using the Send Project Mail feature. 


\title{
CONSENT FORM
}

\author{
The Acute Effects of Cycling with Blood Flow Restriction on \\ Femoral Blood Flow and Muscular Fatigue \\ Matthew A. Kilgas, Steven J. EImer, and Tejin Yoon \\ Department of Kinesiology \& Integrative Physiology \\ Michigan Technological University \\ Houghton, MI 49931 \\ Phone: (906) 487-2324 \\ Fax: (906) 487-0985
}

Email: makilgas@mtu.edu, sjelmer@mtu.edu, and tyoon $@$ mtu.edu

\author{
Introduction \\ of exercise provides a hard workout for muscles without over taxing the heart and lungs.

\section{Exclusion Criteria} \\ Participants will be excluded from the proposed study if they: \\ - Are not between the ages of 18-44 \\ - Are a smoker \\ - Have diabetes \\ - Have any cardiopulmonary disorders, including but not limited to hypertension \\ - Any implanted devices such as but not limited to a pacemaker or pain pump \\ - Any skin or derma logical disorders \\ - Any neurological disorders \\ - Females using contraceptive pills
}

You are invited to participate in a research study being conducted by Dr. Steven Elmer, a faculty member in the Department of Kinesiology and Integrative Physiology at Michigan Technological University. The purpose of his study is to establish better training guidelines for a new type of exercise called "exercise with blood flow restriction". This type of exercise consists of light intensity cycling (30\% of your maximum) while using an inflatable cuff (similar to a tourniquet) to restrict the blood coming back from the muscles to the heart. This type

\section{Experimental Protocol and Brief Risk Summary}

Human Neuromechanics Lab (Student Development Complex Room 121A):

Orientation Visit: We will ask you to attend a required orientation session prior to participating in this research study. During this visit, the procedures and equipment will be explained to you and you will be able to ask questions about what you will do. You will also be asked to fill out a physical activity assessment questionnaire. During this study we will ask you to participate 1 familiarization session, and 4 exercise sessions. These sessions include: 1) Light cycling at 30\% peak aerobic power without blood flow restriction, 2) Light cycling with moderate blood flow restriction, 3) Light cycling with high blood flow restriction, and 4) Heavy cycling without blood flow restriction.

\section{Familiarization session}

Limb Blood Flow: You will be positioned comfortably on an examination table, and a small round-shaped ultrasound probe will be placed on your skin over the middle part of your thigh. This device will enable us to measure blood flow. Ultrasound machines are used in clinical settings and there is minimal risk involved. You may experience some redness on your skin resulting from the contact of the ultrasound probe and gel. However,

\begin{tabular}{l|r|r|} 
Michigan Tech & Approved on: & \multicolumn{1}{|c|}{$07-31-2017$} \\
\hline IRB APPROVAL & Expires on: & $07-30-2018$ \\
\hline Study number: & $\begin{array}{l}1082133-2, \\
\text { M1625 }\end{array}$ \\
\hline
\end{tabular}


this is only temporary and should resolve quickly. Although the risk of an allergic reaction (itching, redness, small raised bumps, and/or burning, on and near the area of contact) is rare, the risk is possible. Symptoms are generally short-lived (few days to few weeks) and you should seek immediate medical attention in case of a severe reaction. To prevent cross contamination, these procedures will not be performed on any participant with known or suspected infection in the body region to be tested, and the ultrasound machine and will be cleaned after each use and disinfected after each participant.

Occlusion Pressure: An inflatable cuff will be placed around your leg. The researcher will inflate the cuff until blood flow is no longer detected. The research will then immediately release the pressure in the cuff. This procedure is similar to having blood pressure taken. This test does not have any associated risks, however the cuff may be uncomfortable, but it will only last a few seconds. To prevent cross contamination, these procedures will not be performed on any participant with known or suspected infection in the body region to be tested, and the occlusion cuff will be cleaned after each use and disinfected after each participant.

Electrical Activity: Electromyography is method to measure the electrical activity of the muscles. The researcher will place small sensors over your arm and leg muscles. For these procedures, slight skin irritation may occur from the small sensors placed on the muscles. Although the risk of an allergic reaction (itching, redness, small raised bumps, and/or burning, on and near the area of contact) is rare, the risk is possible. Symptoms are generally short-lived (few days to few weeks) and you should seek immediate medical attention in case of a severe reaction. The supplier, Noraxon USA Inc., states that they have had no reports of allergic reactions to their electrodes.

Oxygen Content: A NIRS system will be used to detect changes in oxygen in your blood. This is a non-invasive measurement simply obtained by sending near infrared light into the tissue and recording the amount of light that is transmitted through the tissue with a receiving electrode. A NIRS electrode will be placed on the thigh or forearm and secured to the skin with double sided tape. An ace bandage will be used to help secure the electrode and minimize movement on the skin. NIRS does not pose any risk other than perhaps some discomfort of having the device attached to your arm or leg for the duration of the experiment.

Muscle Function: You will be instructed to perform brief max effort muscle contractions with your knee muscles on a device that is similar to standard weight lifting equipment. Prior to conducting these contractions you will warm-up with 10 minutes of low intensity cycling at a self-selected pace. All of these muscle contractions are short ( 5 seconds) and you will likely experience only minimal discomfort. During and immediately after these contractions, a brief electrical stimulation will be applied using an electrical stimulator to your thigh muscle to activate it. It can be uncomfortable and may be painful. The degree of pain is generally rated as moderate i.e. about 4 ( 0 is no pain, and 10 is the worst imaginable pain). It is often described as a sensation similar to that of a low-intensity fist punch to the affected area, and there is no long term risk with these procedures.

Maximal Power Testing: You will cycle at maximal intensity for approximately 5 secs. During a test of this nature, blood pressure and heart rate are expected to elevate to near maximal values. Although the risk of stroke or heart attack is extremely rare, especially in healthy individuals, the risk is possible for anyone exercising, including this exercise you will perform.

Muscle Endurance: You will perform a cycling test for 5-10 minutes. The resistance will increase until you can no longer continue. For this test you will experience significant fatigue in your leg muscles similar to lifting weights, or running. You might also feel a little nauseous and light headed which is not uncommon. To reduce

\begin{tabular}{c|r|l|} 
Michigan Tech & Approved on: & $07-31-2017$ \\
\cline { 2 - 3 } IRB APPROVAL. & Expires on: & $07-30-2018$ \\
\cline { 2 - 3 } & Study number: & $\begin{array}{l}1082133-2 \\
\text { M1625 }\end{array}$ \\
\hline
\end{tabular}


this feeling a cooling fan will be used to help keep you cool and water will be given at the end of test. Although the risk of stroke or heart attack is extremely rare, that the risk is possible for anyone exercising.

\section{Exercise sessions}

Muscle Function: The same risks describe above for the familiarization session also apply here.

Blood Flow Restricted Exercise: this exercise is widely used in Japan and is now emerging in the United States. This form of training, which involves lifting light weights while partially occluding blow flow returning from the working muscles, has been successfully utilized with healthy active individuals, individuals recovering from ACL injury, and older adults. The most common risks are bruising at the site of the tourniquet cuff (incidence $=13.1 \%$ ) and temporary numbness $(1.3 \%)$. More serious side effects include: blood clots in the leg veins $(0.055 \%)$, Stroke $(0.008 \%)$, extreme muscle damage $(0.008 \%)$ and blood clots in the lungs $(0.008 \%)$. Although these events are extremely rare, it should be noted that determining the relative safety of blood flow restriction requires a detailed study of several potential outcomes, and we acknowledge that unforeseen risks do exist. To minimize these risks even further we will limit the training to approximately 15 minutes and the pressure in the cuff will be released between exercises.

Lactate: A small drop of blood with be obtained from your earlobe, which could give rise to mild pain, possible bruising, and a very slight chance of infection. All investigators have completed the Michigan Tech bloodborne pathogens training.

General safety: To further minimize the risks, only healthy individuals 18-44 years of age will be recruited for this investigation. Furthermore, all safety precautions will be followed and the laboratory is equipped with a first aid kit and an AED is located one floor above the laboratory. Participants will be asked how they are feeling throughout exercise protocols. If they give any indication of abnormal stress or injury, the protocol will be stopped. In the event of any incident, we will call Michigan Tech EMS and/or 911 for emergency help.

\section{BENEFITS}

We cannot promise any direct benefit for taking part in this study. You will, however, be provided with results from strength and performance testing. In addition, the results may help researchers and clinicians develop better exercise training programs.

\section{COMPENSATION}

You will not be compensated for your participation in this study.

\section{COST OF PARTICIPATION}

There will be no cost to you for participating in this research.

\section{RESEARCH RELATED INJURY}

In the event of physical and/or mental injury resulting from participation in this study, Michigan Technological University does not provide any medical, hospitalization or other insurance for participants in this study, nor will Michigan Technological University provide any medical treatment or compensation for any injury sustained as a result of participation in this study, except as required by law. If you are taking medications, it is your responsibility to consult with your physician regarding your participation in this study. Do not volunteer for this study if you have been instructed to abstain from this study by a physician. Any problems you experience throughout this study should be discussed immediately with your physician.

\begin{tabular}{c|r|l|}
\multirow{3}{*}{\begin{tabular}{c|c|} 
Michigan Tech \\
IRB APPROVAL
\end{tabular}} & Approved on: & $07-31-2017$ \\
\cline { 2 - 3 } & Expires on: & $07-30-2018$ \\
\cline { 2 - 3 } & Study number: & $\begin{array}{l}1082133-2 \\
\text { M1625 }\end{array}$ \\
\cline { 2 - 3 } & &
\end{tabular}




\section{CONFIDENTIALY OF RECORDS}

We will treat your identity with professional standards of confidentiality. The data from this study may be published, but your identity will not be shown. Paper files with your information and corresponding six digit code will be stored in a locked file cabinet in Dr. Elmer's office (Student Development Complex P105A) for three years after this study is completed. The Michigan Technological University Institutional Review Board (IRB) reserves the right to inspect both the research data collected and your experimental records.

\section{WITHDRAWAL}

Your participation is voluntary. Dr. Steven Elmer, Dr. Yoon, or Mr. Matthew Kilgas will answer any questions you have about the study. Any significant findings which develop during the course of the study which in our opinion may affect your desire to participate will be provided to you as soon as possible. Again, we will ask you to complete one familiarization session, and four exercise sessions, but please note that you are free to withdraw your consent and stop participating at any time and for any reason. This includes the right to withdraw during the actual test.

\section{PARTICIPANT'S RIGHTS INFORMATION}

The Michigan Tech Institutional Review Board has reviewed my request to conduct this project. If you have any concerns about your rights in this study, please contact the Office of Compliance, Integrity, and Safety at 906487-2902 or email IRB@mtu.edu.

I, me an opportunity to ask questions and I wish to voluntarily participate in this study.

\begin{tabular}{l|r|l|}
\multicolumn{1}{c}{ Michigan Tech } & Approved on: & $07-31-2017$ \\
\hline IRB APPROVAL & Expires on: & $07-30-2018$ \\
\cline { 2 - 3 } & Study number: & $\begin{array}{l}1082133-2 \\
\text { M1625 }\end{array}$ \\
\cline { 2 - 3 } & &
\end{tabular}




\section{A.3 Study 3}

\section{Michigan \\ 101. Technological 1885 University}

Compliance, Integrity, and Safety

302 Lakeshore Center | 1400 Townsend Drive Houghton. MI 49931-1295 $906-487-2902$ |f. 906-487-2245
DATE:

TO:

FROM:

RE:

TITLE:

SUBMISSION TYPE:

STATUS:
March 2, 2018

Steven Elmer, PhD, KIP

Joanne Polzien, Executive Director

M1692, [1175311-3]

Exercise Training with Blood Flow Restriction

Response/Follow-Up

New Project, APPROVED

The Institutional Review Board (IRB) has reviewed and approved your research application as compliant with all applicable sections of the federal regulations, Michigan law, and Michigan Tech IRB policies and procedures. All research must be conducted in accordance with this approved submission.

APPROVAL DATE: March 2, 2018

EXPIRATION DATE: March 1, 2019

A pdf of this signed memo and any stamped approved documents, if applicable, have been placed in the review details under "board documents" for this project.

This approval applies only for this project, and only under the conditions and procedures described in the application. If/when changes become necessary but are not limited to: changes in protocol, personnel, study location, participant recruitment, etc., as set forth in this approval, you must follow the INSTRUCTIONS and submit the FORM for Change Request during approval found in the IRBNet Library. You must receive notification of approval PRIOR to implementing the change(s).

Approvals are granted for up to a one year period. You are responsible for submitting requests for continuation in advance of the expiration date for each year of the project. You will need to request a continuation six weeks prior to the end date indicated above. It is very important that the expiration date is not missed. Failure to submit annual review materials on time will result in the termination of this protocol on the expiration date listed above.

Please note the following in order to comply with federal regulations and IRB policy:

1. Please remember that informed consent is a process beginning with a description of the study and assurance of participant understanding followed by a signed consent form. Informed consent must continue throughout the study via a dialogue between the researcher and research participant. Federal regulations require each participant receive a copy of the signed consent document.

2. Individual identification of human subjects in any publication is an invasion of privacy. Before beginning a project involving human subjects, and only if required, the principal investigator 
must obtain a properly executed informed consent from each subject and/or the person legally responsible for the subject. If a consent form has been reviewed and approved it has been attached with an official date stamp on it. Only copies of the official date stamped informed consent are to be distributed to participants relating to this project. If any changes or modifications are needed regarding this form, you must first submit the revised document for review and approval prior to use. The PI must retain informed consent forms on file for at least three years after the end of the project.

3. The approved project will be subject to periodic review. This review will consist of consulting with the $\mathrm{PI}$ and examining the appropriate project records. All required research records must be securely retained in either paper or electronic format for a minimum of three years following the closure of the approved study. This includes signed consent documents from all participants.

4. All Unanticipated Problems / Serious Adverse Events to participants or other parties affected by the research must be reported to this office within two days of the event occurrence. All instances of non-compliance or complaints regarding this study must be reported to this office in a timely manner. Please use the INSTRUCTIONS and FORM: Unanticipated Problem / Serious Adverse Event Form found both on our web site and the IRBNet Library.

If you have any questions, please contact the Compliance, Integrity, and Safety Office at 906.487.2902 or send your message via email through IRBNet using the Send Project Mail feature. 


\author{
Exercise Training with Blood Flow Restriction \\ Matthew A. Kilgas, Steven J. Elmer \\ Department of Kinesiology \& Integrative Physiology \\ Michigan Technological University \\ Houghton, MI 49931 \\ Phone: (906) 487-2324 \\ Fax: (906) 487-0985 \\ Email: makilgas@mtu.edu, sielmer@mtu.edu
}

\title{
Introduction
}

You are invited to participate in a research study being conducted by Professor Steven Elmer, a faculty member in the Department of Kinesiology and Integrative Physiology at Michigan Technological University. Mr. Matthew Kilgas, a doctoral student in the Integrative Physiology program, will also be assisting with this study. The purpose of this study is to establish better exercise prescription guidelines for a new type of exercise called "exercise with blood flow restriction". This type of exercise consists of light to moderate intensity exercise (30$40 \%$ of your maximum) while using an inflatable cuff (similar to a tourniquet) to restrict the blood coming back from the muscles to the heart. This form of exercise provides a hard workout for muscles without over taxing the heart and lungs. For this study we will evaluate your muscle strength and performance before and after 6 consecutive weeks exercise training with blood flow restriction.

\section{Exclusion Criteria}

Participants will be excluded from the proposed study if they:

- Are not between the ages of 18-44

- Are a smoker

- Have diabetes

- Have any cardiopulmonary disorders, including but not limited to hypertension

- Any implanted devices such as but not limited to a pacemaker or pain pump

- Any skin or dermatological disorders

- Any neurological disorders

- Are a female using contraceptive pills

- Have any lower-body injuries that affect their ability to exercise

- Have engaged in regular exercise more than 3 day/wk in last 60 days

\section{Experimental Protocol and Brief Risk Summary}

Human Performance Laboratory (Student Development Complex Room P105):

Orientation Visit: We will ask you to attend a required orientation session prior to participating in this research study. During this visit, the procedures and equipment will be explained to you, and you will be able to ask questions about what you will do. For this study we will ask you to complete a total of 24 laboratory visits. Specifically, there will be 3 pre-training sessions, 18 exercise sessions with blood flow restriction, and 3 posttraining sessions. At the end of the orientation visit you will be randomly placed into one of the following exercise

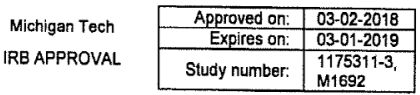


training groups:

This study involves two exercise training groups

- Group A - bicycling exercise

- Group B - weight lifting exercise

Again, you will either perform bicycling or weight lifting exercise for 6 consecutive weeks.

\section{Pre-training Sessions (3 Visits)}

Muscle Size: You will be positioned comfortably on an examination table, and a small round-shaped ultrasound probe will placed on your skin over the middle part of your thigh. This device will enable us to measure the size of your quadriceps muscles. Ultrasound machines are used in clinical settings and there is minimal risk involved. You may experience some redness on your skin resulting from the contact of the ultrasound probe and gel. However, this is only temporary and should resolve quickly. Although the risk of an allergic reaction (itching, redness, small raised bumps, and/or burning, on and near the area of contact) is rare, the risk is possible. Symptoms are generally short-lived (few days to few weeks) and you should seek immediate medical attention in case of a severe reaction.

Muscle Strength: You will be seated on a specialized laboratory weight lifting machine similar to a knee extension machine that you might use in the gym. Your right lower leg will be strapped to the lever arm of the device. You will be asked to push as hard as you can against the arm and straighten your knee. The machine will provide resistance and measure your quadriceps muscle strength. This test will require several brief maximal effort muscle contractions ( 5 seconds). You will also be asked to perform two more common strength tests (leg press and knee extension) on regular weight room equipment. The research staff will help you select a moderate to heavy weight and ask you to lift that weight with your legs for 1 set of $8-12$ repetitions. For all of these strength tests you will likely experience only minimal discomfort. There is, however, a risk of muscle strains, or other soft tissue injuries. To minimize the risk of injury with this exercise, you will be instructed on how to perform each exercise with the proper technique. For these tests you may experience fatigue in your leg muscles similar to lifting weights, running, or playing team sports.

Muscle Power: You will pedal a bicycle as fast and as hard as you can for 30 seconds. For this test the resistance will increase to $8 \%$ of your body weight (very difficult). You will be asked to continue cycling as fast and hard as possible for the entire 30 seconds. During a test of this nature, blood pressure and heart rate are expected to elevate to near maximal values. Although the risk of stroke or heart attack is extremely rare, especially in healthy individuals, the risk is possible for anyone exercising, including this exercise you will perform.

Endurance Fitness: You will pedal a bicycle for 5-10 minutes. The resistance will gradually increase until you can no longer maintain the required force output. This will feel similar to riding a bicycle up a very long hill or climbing 10 flights of stairs in tall building. For this test you will experience significant fatigue in your leg muscles similar to lifting weights or running. You might also feel a little nauseous and light headed which is not uncommon. To reduce this feeling a cooling fan will be used to help keep you cool and water will be given at the end of test. During the test we will also check your oxygen rate, heartbeat, blood pressure, breathing rate, and continuously ask you how you are feeling. The test will end immediately if you chose to stop at anytime, feel dizzy or confused, experience chest pain or shortness of breath, or have muscle cramps or fatigue. Also, if your blood pressure drops too much or your skin color changes we will end the test immediately. Although the risk of stroke or heart attack is extremely rare, that the risk is possible for anyone exercising. A small drop of blood will

Michigan Tech

IRB APPROVAL

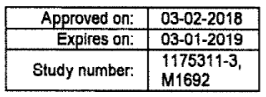


be drawn from your fingertip by a lancet. This could give rise to mild pain, possible bruising, and a very slight chance of infection. Blood samples will be obtained using sterile single-use lancets and a spring-loaded lancet device. All investigators have completed the Michigan Tech bloodborne pathogens training.

Blood Flow Restriction Cuff Pressure: You will be positioned comfortably on an examination table, and a small round-shaped ultrasound probe will placed on your skin over the middle part of your thigh. This device will enable us to measure blood flow. Ultrasound machines are used in clinical settings and there is minimal risk involved. You may experience some redness on your skin resulting from the contact of the ultrasound probe and gel. However, this is only temporary and should resolve quickly. Although the risk of an allergic reaction (itching, redness, small raised bumps, and/or burning, on and near the area of contact) is rare, the risk is possible. Symptoms are generally short-lived (few days to few weeks) and you should seek immediate medical attention in case of a severe reaction. We will again use the ultrasound to measure occlusion pressure by placing a cuff around the upper part of your thigh. The investigator will inflate the occlusion cuff starting at $100 \mathrm{mmHg}$ and increasing the pressure until no blood flow can be detected in the superficial femoral artery using the Doppler Ultrasound. The investigator will then slowly deflate the occlusion cuff until blood flow resumes, this procedure is similar to measuring blood pressure. This test does not have any associated risks; however, the cuff may be uncomfortable but only lasts a few seconds.

Exercise sessions (18 Visits)

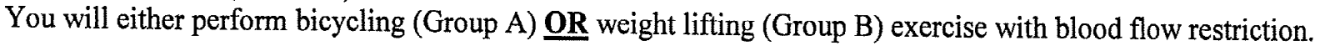

Group A-Bicycling Exercise Training: You will be asked to come into the laboratory for training $3 \mathrm{x} / \mathrm{week}$ for 6 weeks. Each training session will last $\sim 30$ minutes. You will be asked to complete a total of 8 cycling intervals on a stationary bike. Each interval will last 2 minutes. The resistance provided by the bicycle will be moderate ( $40 \%$ of maximum). To minimize the risk of muscular strains or cardiovascular events, training will always include a warm-up and cool-down. We will also provide a cooling fan and water.

Group B Weight Lifting Training: You will be asked to come into the laboratory for training $3 \mathrm{x} /$ week for 6 weeks. Each training session will last $\sim 30$ minutes. You will be asked to complete 6 weight lifting sets (105 repetitions) on common weight room equipment (leg press and knee extension). The weight that you lift will be light ( $30 \%$ of maximum). To minimize the risk of muscular strains or cardiovascular events, training will always include a warm-up and cool-down. We will also provide a cooling fan and water.

Blood Flow Restriction Exercise: During training, a blood pressure cuff will be inflated to $80 \%$ of the occlusion pressure, assessed during the pre-training visits. Note that, when exercising with a blood pressure cuff you will experience substantial fatigue in your leg muscles similar to that when lifting heavy weights. The most common side effects for this exercise are bruising at the site of the blood pressure cuff and temporary numbness. More serious side effects include blood clots in the leg veins $(0.055 \%)$, stroke $(0.008 \%)$, extreme muscle damage $(0.008 \%)$, and blood clots in the lungs $(0.008 \%)$. Although these events are extremely rare. It should be noted that determining the relative safety of blood flow restriction exercise requires a detailed study of several potential outcomes, and we acknowledge that unforeseen risks do exist. To minimize these risks even further the blood flow restriction will not be applied continuously as the blood pressure cuff pressure will be release between exercises, and not applied during the warm-up or cool-down.

Cardiovascular Measures: You will wear a pressurized finger cuff during exercise that will allow us to measure your heart rate, and blood pressure. The cuff can be uncomfortable, and could cause light tingling feeling in your

\begin{tabular}{l|l|l|} 
Michigan Tech & Approved on: & 03-02-2018 \\
\cline { 2 - 3 } IRB APPROVAL & Expires on: & $03-01-2019$ \\
\hline Study number: & $\begin{array}{l}1175311-3, \\
\text { M1692 }\end{array}$ \\
\hline
\end{tabular}



fingertip. These symptoms should quickly subside when the pressure is released. There is no known risk in healthy
individuals.

\section{Post-Training Session (3 visits)}

At the end of the 6 weeks your muscle size, strength, power and endurance fitness will be assessed in the same manner as listed in the pre-training section.

\section{General Safety Statement}

To further minimize the risks, only healthy individuals 18-44 years of age will be recruited for this study. Furthermore, all safety precautions will be followed and the laboratory is equipped with a first aid kit. An AED is located just down the hall as well as one floor above the laboratory. You will be asked how you are feeling throughout exercise protocols. If you give any indication of abnormal stress or injury, the protocol will be stopped. In the event of any incident, we will call we will call 911 and then MTU EMS.

\section{BENEFITS}

We cannot promise any direct benefit for taking part in this study. You will, however, be provided with results from strength and performance testing. In addition, the results may help researchers and clinicians develop better exercise training programs.

\section{COMPENSATION}

There will be no compensation for your participation in this research.

\section{COST OF PARTICIPATION}

There will be no cost to you for participating in this research.

\section{RESEARCH RELATED INJURY}

In the event of physical and/or mental injury resulting from participation in this study, Michigan Technological University does not provide any medical, hospitalization or other insurance for participants in this study, nor will Michigan Technological University provide any medical treatment or compensation for any injury sustained as a result of participation in this study, except as required by law. If you are taking medications, it is your responsibility to consult with your physician regarding your participation in this study. Do not volunteer for this study if you have been instructed to abstain from this study by a physician. Any problems you experience throughout this study should be discussed immediately with your physician.

\section{CONFIDENTIALY OF RECORDS}

We will treat your identity with professional standards of confidentiality. The data from this study may be published, but your identity will not be shown. Paper files with your information and corresponding six digit code will be stored in a locked file cabinet in Dr. Elmer's office (Student Development Complex P105A) for three years after this study is completed. The Michigan Technological University Institutional Review Board (IRB) reserves the right to inspect both the research data collected and your experimental records.

\section{WITHDRAWAL}

Your participation is voluntary. Mr. Matthew Kilgas will answer any questions you have about the study. Any significant findings which develop during the course of the study which in our opinion may affect your desire to participate will be provided to you as soon as possible. Again, we will ask you to complete 8 weeks of testing but please note that you are free to withdraw your consent and stop participating at any time and for any reason.

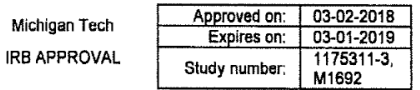


This includes the right to withdraw during the actual test. If you withdraw yourself during the actual testing, you will still be compensated for that session.

\section{PARTICIPANT'S RIGHTS INFORMATION}

The Michigan Tech Institutional Review Board has reviewed my request to conduct this project. If you have any concerns about your rights in this study, please contact the Office of Compliance, Integrity, and Safety at 906487-2902 or email IRB@mtu.edu.

I, , have read through this consent form. The investigator provided me an opportunity to ask questions and I wish to voluntarily participate in this study. 


\section{A.4 Dissertation Project}

\section{Michigan \\ 31. Technological \\ 18 8 5 University}

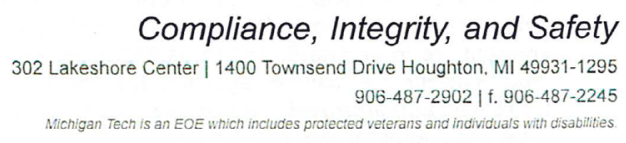

Compliance, Integrity, and Safety

DATE:

December 5, 2016

TO:

Steven Elmer, PhD, KIP

FROM:

Joanne Polzien, Executive Director

RE:

M1542, [980620-3]

TITLE:

Restoring Quadriceps Function Following ACL Reconstruction with Blood Flow Restricted Exercise

SUBMISSION TYPE:

Revision

STATUS:

New Project, APPROVED

The Institutional Review Board (IRB) has reviewed and approved your research application as compliant with all applicable sections of the federal regulations, Michigan law, and Michigan Tech IRB policies and procedures. All research must be conducted in accordance with this approved submission.

APPROVAL DATE: December 5, 2016

EXPIRATION DATE: December 4, 2017

A pdf of this signed memo and any stamped approved documents, if applicable, have been placed in the review details under "board documents" for this project.

This approval applies only for this project, and only under the conditions and procedures described in the application. If/when changes become necessary but are not limited to: changes in protocol, personnel, study location, participant recruitment, etc., as set forth in this approval, you must follow the INSTRUCTIONS and submit the FORM for Change Request during approval found in the IRBNet Library. You must receive notification of approval PRIOR to implementing the change(s).

Approvals are granted for up to a one year period. You are responsible for submitting requests for continuation in advance of the expiration date for each year of the project. You will need to request a continuation six weeks prior to the end date indicated above. It is very important that the expiration date is not missed. Failure to submit annual review materials on time will result in the termination of this protocol on the expiration date listed above.

Please note the following in order to comply with federal regulations and IRB policy:

1. Please remember that informed consent is a process beginning with a description of the study and assurance of participant understanding followed by a signed consent form. Informed consent must continue throughout the study via a dialogue between the researcher and research participant. Federal regulations require each participant receive a copy of the signed consent document. 
2. Individual identification of human subjects in any publication is an invasion of privacy. Before beginning a project involving human subjects, and only if required, the principal investigator must obtain a properly executed informed consent from each subject and/or the person legally responsible for the subject. If a consent form has been reviewed and approved it has been attached with an official date stamp on it. Only copies of the official date stamped informed consent are to be distributed to participants relating to this project. If any changes or modifications are needed regarding this form, you must first submit the revised document for review and approval prior to use. The PI must retain informed consent forms on file for at least three years after the end of the project.

3. The approved project will be subject to periodic review. This review will consist of consulting with the PI and examining the appropriate project records. All required research records must be securely retained in either paper or electronic format for a minimum of three years following the closure of the approved study. This includes signed consent documents from all participants.

4. All Unanticipated Problems / Serious Adverse Events to participants or other parties affected by the research must be reported to this office within two days of the event occurrence. All instances of non-compliance or complaints regarding this study must be reported to this office in a timely manner. Please use the INSTRUCTIONS and FORM: Unanticipated Problem / Serious Adverse Event Form found both on our web site and the IRBNet Library.

If you have any questions, please contact the Compliance, Integrity, and Safety Office at 906.487.2902 or send your message via email through IRBNet using the Send Project Mail feature. 


\title{
Restoring Function After ACL Reconstruction
}

\author{
Matthew A Kilgas and Steven J. Elmer \\ Department of Kinesiology \& Integrative Physiology \\ Michigan Technological University \\ Houghton, MI 49931 \\ Phone: (906) 487-2324 \\ Fax: (906) 487-0985 \\ Email: makilgas@mtu.edu and sielemer@mtu.edu
}

\section{Introduction}

You are invited to participate in a research study being conducted by Dr. Steven Elmer, a faculty member in the Department of Kinesiology and Integrative Physiology at Michigan Technological University. The purpose of this study is to evaluate the effectiveness of a home-based exercise program to improve quadriceps muscle size and strength and reduce chronic asymmetry seen in individuals who have previously had an ACL reconstruction and have been discharged from a rehabilitation program.

Prior to participating in the study, you will have to provide documentation from your primary care physician or physical therapist stating that you are able to participate in lower extremity resistance training.

\author{
Inclusion Criteria \\ - 18-70 years of age \\ - Have had ACL reconstruction > 2 years ago \\ Exclusion Criteria \\ - Have any physical limitations as a result of lower extremity injury \\ - Are a smoker \\ - Have diabetes \\ - Have any cardiopulmonary disorders, including but not limited to hypertension
}

\section{Experimental Protocol and Brief Risk Summary}

Human Performance Laboratory (Student Development Complex Room P105):

Orientation Visit: We will ask you to attend a required orientation session prior to participating in this research study. During this visit, the procedures and equipment will be explained to you, and you will be able to ask questions about what you will do. For this study we will ask you to participate in an 8 week exercise program (14 total visits to the laboratory) consisting of light weight lifting exercise.

\section{Baseline testing (3 visits)}

Muscle size: You will be positioned comfortably on an examination table, and a small round-shaped ultrasound probe will placed on your skin over the middle part of your thigh. This device will enable us to measure the size of your quadriceps muscles. Ultrasound machines are used in clinical settings and there is minimal risk involved. You may experience some redness on your skin resulting from the contact of the ultrasound probe and gel. However, this is only temporary and should resolve quickly. Although the risk of an allergic reaction (itching, redness, small raised bumps, and/or burning, on and near the area of contact) is rare, the risk is possible. Symptoms are generally short-lived (few days to few weeks) and you should seek immediate medical attention in case of a severe reaction.

\begin{tabular}{l|l|l|} 
Michigan Tech & Approved on: & $12-05-2016$ \\
\cline { 2 - 3 } IRB APPROVAL & Expires on: & $12-04-2017$ \\
\cline { 2 - 3 } & Study number: & $\begin{array}{l}980620-3, \\
\text { Mi542, }\end{array}$ \\
\hline
\end{tabular}


Muscle strength: You will be instructed to perform brief max effort muscle contractions with your knee muscles on a device that is similar to standard weight lifting equipment. Prior to this test you will warm-up with 10 minutes of low intensity cycling at a self-selected pace. All of these muscle contractions are short ( 5 seconds) and you will likely experience only minimal discomfort.

Muscle endurance: You will perform a series of dynamic endurance tests consisting of 1) knee kicking and 2) single-leg cycling until fatigue ( $\sim-10$ minutes). Prior to these tests you will warm-up with 10 minutes of low intensity cycling at a self-selected pace. For the knee kicking you will be asked to repeatedly extend your knee on a knee kicking machine against heavier resistance until fatigue. For the single-leg cycling you will be cycle on an ergometer with only one leg at a time. The resistance will increase until you can no longer maintain the required force output. For both of these tests you will experience significant fatigue in your leg muscles similar to lifting weights, or running. There is a risk of reinjuring your ACL, or other soft tissue injuries with knee kicking exercise. To minimize the risk of injury with this exercise, intensity will be keep below $50 \%$ of your 1 repetition maximum. You might also feel a little nauseous and light headed which is not uncommon. To reduce this feeling a cooling fan will be used to help keep you cool and water will be given at the end of test. Although the risk of stroke or heart attack is extremely rare, that the risk is possible for anyone exercising.

Functional Capacity: You will instructed to perform a one-legged vertical jump. Prior to this test you will warmup with 10 minutes of low intensity cycling at a self-selected pace. Your standing reach will be recorded for both the right and left sides. You will then be asked to jump off of a specified limb, touch the wall, and land on the same limb. The opposite limb is not used during the test. Two trials will be performed on each leg. To minimize the risk of injury an investigator will spot and support you to ensure safe landing.

\section{Week Exercise Program (8 visits) \\ Before the exercise program starts you will be placed in either a Group A or Group B.}

Group B: You will train for 8 weeks. During training a blood pressure cuff will be inflated to $50 \%$ of the occlusion pressure, assessed during the baseline measures. The program will consist of home-based walking and weight lifting exercises employing body weight, ankle weights and resistance bands to load your thigh muscles. The exercises include 3 sets of repetitions until failure of half squat, and leg extension with ankle weights or resistance bands ( $25 \%$ of your 1 repetition maximum) with 30 seconds rest between. Followed by 3 sets of 2 minute of walking or stepping-in-place with 10 seconds rest between. During the exercises you will inflate a blood pressure cuff to a prescribed pressure. Performing the exercise with a blood pressure cuff inflated will make the muscles work hard without placing additional load on the knee joint. You will be instructed how to perform these exercises by the research group. Each week you will be asked to come into the exercise physiology laboratory on time to perform the exercise routine under supervision and then perform the exercises at home four more times each week. . Note that, when exercising with a blood pressure cuff you will experience substantial fatigue in your leg muscles similar to that when lifting heavy weights. The most common side effects for this exercise are bruising at the site of the blood pressure cuff and temporary numbness. More serious side effects including nerve damage are possible. In the event of any discomfort you will be instructed to stop exercising and quickly deflate the blood pressure cuff. Moreover to minimize the risk of injury exercise training will last approximately 15 minutes and the blood flow restriction will not be applied continuously as the blood pressure cuff pressure will be release between exercises.

\begin{tabular}{l|r|l|} 
& \\
Michigan Tech & Approved on: & $12-05-2016$ \\
\cline { 2 - 3 } IRB APPROVAL & Explies on: & $12-04-2017$ \\
\cline { 2 - 3 } & Study number: & \multicolumn{2}{l}{\begin{tabular}{l} 
M150620-3, \\
\hline
\end{tabular}} &
\end{tabular}


Post-Exercise Program Testing (3 visits)

At the end of the 8 weeks your muscle size, strength and functional capacity will be assessed in the same manner as listed in the baseline testing section.

\section{BENEFITS}

We cannot promise any direct benefit for taking part in this study. You will, however, be provided with results from strength and performance testing. In addition, the results may help researchers and clinicians develop better exercise training programs.

\section{COMPENSATION}

You will be compensated $\$ 12.50$ per visit and thus would receive a total of $\$ 175$ for completing all 14 visits (3 visits for baseline testing, 8 visits for the 8 week exercise program, and 3 visits for post-exercise program testing) for the study. If you withdraw from the study you will receive compensation for the number of visits completed. To be compensated you will need to complete a Receipt of Compensation Form (including a W-9), and may also need to report this compensation as income when filing taxes to the IRS.

\section{COST OF PARTICIPATION}

There will be no cost to you for participating in this research.

\section{RESEARCH RELATED INJURY}

In the event of physical and/or mental injury resulting from participation in this study, Michigan Technological University does not provide any medical, hospitalization or other insurance for participants in this study, nor will Michigan Technological University provide any medical treatment or compensation for any injury sustained as a result of participation in this study, except as required by law. If you are taking medications, it is your responsibility to consult with your physician regarding your participation in this study. Do not volunteer for this study if you have been instructed to abstain from this study by a physician. Any problems you experience throughout this study should be discussed immediately with your physician.

\section{CONFIDENTIALY OF RECORDS}

We will treat your identity with professional standards of confidentiality. The data from this study may be published, but your identity will not be shown. Paper files with your information and corresponding six digit code will be stored in a locked file cabinet in Dr. Elmer's office (Student Development Complex P105A) for three years after this study is completed. The Michigan Technological University Institutional Review Board (IRB) reserves the right to inspect both the research data collected and your experimental records.

\section{WITHDRAWAL}

Your participation is voluntary. Mr. Matthew Kilgas will answer any questions you have about the study. Any significant findings which devclop during the course of the study which in our opinion may affect your desire to participate will be provided to you as soon as possible. Again, we will ask you to complete 8 weeks of testing but please note that you are free to withdraw your consent and stop participating at any time and for any reason. This includes the right to withdraw during the actual test. If you withdraw yourself during the actual testing, you will still be compensated for that session.

\begin{tabular}{l|l|l|} 
Michigan Tech & Approved on: & $12-05-2016$ \\
\cline { 2 - 3 } IRB APPROVAL & Expires on: & $12-04-2017$ \\
\cline { 2 - 3 } & Study number: & $980520-3$, \\
\hline
\end{tabular}


PARTICIPANT'S RIGHTS INFORMATION

The Michigan Tech Institutional Review Board has reviewed my request to conduct this project. If you have any concerns about your rights in this study, please contact the Office of Compliance, Integrity, and Safety at 906487-2902 or email IRB@mtu.edu.

I, , have read through this consent form. The investigator provided me an opportunity to ask questions and I wish to voluntarily participate in this study.

Signature of Participant

Signature of Investigator
Date

Date
Michigan Tech

IRB APPROVAL

\begin{tabular}{|r|l|}
\hline Approved on: & $12-05-2016$ \\
\hline Expires on: & $12-04-2017$ \\
\hline Study number: & $980620-3$, \\
\hline
\end{tabular}




\section{B Submitted Manuscripts}

\section{B.1 Home-Based Exercise with Blood Flow Restriction to Restore Limb Symmetry Following ACL Reconstruction}

\section{B.1.1 Abstract}

Study Design: Controlled laboratory study, longitudinal.

Background: Quadriceps atrophy and weakness can persist for years after anterior cruciate ligament reconstruction (ACLR). Such impairments can limit physical function, increase re-injury risk, and lead to early osteoarthritis.

Objectives: To evaluate the effectiveness of a home-based blood flow restriction (BFR) exercise program to increase quadriceps size and strength several years after ACLR.

Methods: Nine adults with ACLR ( $5 \pm 2$ yrs post-surgery, $\leq 90 \%$ quadriceps symmetry) and nine uninjured controls volunteered. ACLR participants exercised at home for $\sim 25 \mathrm{~min}, 5 \mathrm{x} / \mathrm{wk}$ for $4 \mathrm{wks}$ (bodyweight half-squats, single-leg knee extension, walking). Blood flow in only the ACLR leg was restricted using a thigh cuff inflated to $50 \%$ of limb occlusion pressure. Rectus femoris and vastus lateralis thickness and knee extensor strength were measured before and after training. Baseline and post-training symmetry (ACLR leg/uninvolved leg) indices were compared to uninjured controls. Muscle soreness with the exercise program was assessed using an analog scale $(0-10 \mathrm{~cm})$.

Results: Rectus femoris and vastus lateralis thickness and knee extensor strength in the ACLR leg increased by $11 \pm 5 \%, 10 \pm 6 \%$, and $20 \pm 14 \%$, respectively (all $\mathrm{P}<0.01$ ). Compared to baseline, post-training knee extensor strength symmetry increased from 
$88 \pm 4$ to $99 \pm 5 \%(\mathrm{P}<0.01)$ and did not differ from uninjured controls $(99 \pm 5 \%, \mathrm{P}=0.95)$.

Mean muscle soreness across the 20 exercise training sessions was very low $(0.41 \pm 0.44$ to $0.91 \pm 1.31 \mathrm{~cm})$.

Conclusion: Implementation of BFR exercise at home was feasible, safe, and effective. Results extend upon early post-operative application of BFR exercise for ACLR recovery and demonstrate that BFR can improve quadriceps function long after ACLR.

\section{B.1.2 Introduction}

After an anterior cruciate ligament injury and reconstruction (ACLR) many individuals do not completely regain their quadriceps size $\mathrm{e}^{19,28,48}$ and strength ${ }^{7,14,19,47}$ and only $60 \%$ are able to return to pre-injury activity levels. ${ }^{3}$ Persistent muscle and strength loss in the injured leg gives rise to quadriceps asymmetry which is associated with altered joint loading and gait mechanics, ${ }^{6,34}$ limited physical function, ${ }^{39}$ and increased risk for reinjury. ${ }^{18}$ Moreover, chronic quadriceps asymmetry has the potential to lead to early onset of osteoarthritis ${ }^{33,49}$ thus requiring costly continued medical intervention. Many authors ${ }^{20,21,26}$ have also reported the presence of quadriceps asymmetry several years after ACLR. However, there are few reports documenting the implementation of therapeutic interventions to overcome these persistent impairments, improve quadriceps size and strength, and restore quadriceps symmetry. Therefore, developing effective strategies to improve and restore quadriceps function long after ACLR is not only warranted but is of paramount importance so individuals living with an ACLR can maintain active lives and avoid long-term disability. 
Performing resistance exercise at $65-70 \%$ of one repetition maximum (1RM), 2$3 \mathrm{x} / \mathrm{wk}$, for 8 weeks is sufficient to increase quadriceps size and strength. ${ }^{9}$ This type of exercise, however, is not always possible because the high loads are contraindicated after ACLR as it could add excessive strain on the repaired ligament ${ }^{13}$ and/or irritate the knee joint. Alternatively, implementation of exercise with blood flow restriction (BFR) is emerging as an effective method to build muscle and improve strength in clinical populations with orthopedic limitations (e.g., ACLR, osteoarthritis, total knee arthroplasty). ${ }^{15}$ For this exercise, an inflatable cuff applies mechanical compression to the limb to partially occlude blood flow while much lower loads are used compared to traditional resistance exercise (e.g., 20 vs. $70 \%$ of 1 RM). Evidence44 suggests that BFR can be applied days after ACLR surgery to minimize quadriceps atrophy. Other groups have demonstrated that BFR exercise increases quadriceps muscle size and strength to a greater extent than traditional rehabilitative care following $\mathrm{ACLR}^{32}$ and knee arthroscopy ${ }^{46}$ To date, there are no reports of using BFR exercise years after ACLR.

Following discharge from a supervised rehabilitation protocol, patients are encouraged to continue with a home-based program in order to achieve and maintain optimal function. Continued adherence to this program is crucial, as patients are often permitted to return to limited sport activities with $85-90 \%$ quadriceps and/or limb symmetry ${ }^{5,23,34,39}$ with the expectation that they will successfully regain full symmetry with time. However, limited access to specialized exercise equipment, time constraints, and costs associated with follow-up treatment can pose barriers. Implementation of BFR at home may circumvent these limitations as it can be performed with or without weights 
(i.e., bodyweight only and/or resistance bands), facilitates strength gains faster than traditional training, and involves inexpensive equipment. Successful home-based programs also depend on the individual's motivation, amount of program education received, and previous experience exercising independently. ${ }^{11}$ Thus, implementation of BFR exercise at home years after an ACLR warrants further consideration as it could offer a simple and inexpensive option to aid with long term recovery.

Our purpose for conducting this investigation was to evaluate the effectiveness of a 4 week home-based BFR exercise program to increase quadriceps size, strength, and symmetry several years after ACLR. We envisioned that a home program consisting of bodyweight and walking exercises with BFR would be feasible, safe, and inexpensive. We hypothesized that BFR exercise would stimulate greater improvements in rectus femoris and vastus lateralis muscle thickness and knee extensor strength in the ACLR leg compared to the uninvolved leg (i.e., non-BFR leg). We also hypothesized that quadriceps asymmetry would decrease thus resulting in symmetry levels that would be much closer to those for uninjured controls.

\section{B.1.3 Methods}

\section{Participants}

Nine adults who had undergone an ACLR and nine uninjured controls volunteered to participate in this study (demographics reported in Table 1). Participants were between 18-44yrs of age and were recreationally active which was defined as exercising 3-5x/week for 30min at a moderate intensity. 9 Individuals in the ACLR group 
were eligible to participate if they: 1) had a unilateral ACLR $>2$ yrs ago, 2) completed a post-operative rehabilitation program, 3) were cleared to return to physical activity by their orthopedic surgeon, and 4) exhibited greater than a $10 \%$ difference in their rectus femoris thickness, vastus lateralis thickness, or knee extensor strength between their ACLR and uninvolved leg (i.e., $<90 \%$ symmetry). A physical therapist performed an evaluation to verify that these participants were able to perform the baseline testing and home-based BFR exercise program. Specifically, participants had to display negative signs and symptoms for knee ligamentous laxity, meniscal involvement, and patellar dysfunction. Participants also needed to demonstrate adequate muscular stability through the ACLR leg during a single-leg squat, bilateral vertical jump, and forward, backward, and lateral lunge matrix. Individuals in the uninjured control group were eligible if they had no history of lower-extremity joint surgeries. Experimental procedures used in this investigation were reviewed and approved by the institutional review boards at Michigan Technological University and Northern Michigan University. The procedures were explained and participants provided written informed consent before testing.

\section{Study Overview}

Baseline measures of rectus femoris thickness, vastus lateralis thickness, and single-leg knee extensor strength were assessed in the ACLR and uninjured control groups. Participants in the ACLR group performed BFR exercise at home for $\sim 25 \mathrm{~min}$, 5x/week for 4 weeks. Within one week following the completion of the exercise program rectus femoris thickness, vastus lateralis thickness, and single-leg knee extensor strength were assessed again in the ACLR group. Baseline and post-training symmetry indices for 
ACLR participants (ACLR leg/uninvolved leg) were compared to the baseline values for uninjured controls (nondominant leg/dominant leg).

\section{Home-Based BFR Exercise Program}

Participants in the ACLR group visited the laboratory to complete a familiarization session where they were introduced to the BFR exercise program. Subsequently, each training session performed at home consisted of a series of three exercises (single-leg knee extension, bodyweight half-squats, walking) and took $\sim 25 \mathrm{~min}$ to complete (Figure 1). First, participants performed 3 sets of 30 double-leg bodyweight half squats. Participants were instructed to keep their feet shoulder width apart and lower their body to $\sim 45^{\circ}$ and slowly rise back up. Next, participants performed 3 sets of 30 single-leg knee extensions using their ACLR leg only with resistance bands. The level of resistance was selected by the investigator such that the participant was able to complete all 3 sets. Participants were instructed to move up to the next resistance band when it was no longer challenging to complete all 3 sets. Finally, participants performed 3 sets of 2 min walking intervals at their self-selected walking speed. If they were not able to find a location for sustained walking they substituted this with 3 sets of $2 \mathrm{~min}$ stepping in place intervals. Throughout the training session a $1 \mathrm{~min}$ rest was provided between sets and $2 \mathrm{~min}$ of rest was given between exercises.

During each exercise, only blood flow in the ACLR leg was restricted using an $18 \mathrm{~cm}$ wide aneroid sphygmomanometer (Briggs, Healthcare, Waukegan, IL, USA). The inflatable cuff was wrapped around the thigh at the most proximal location and pressure 
was inflated to $50 \%$ of the pressure required to completely occlude blood flow in the femoral artery (i.e., limb occlusion pressure, described below). The cuff remained inflated during the $1 \mathrm{~min}$ rest between sets but was deflated during the $2 \mathrm{~min}$ of rest between exercises. Note that, during the rest period participants returned to a seated position to carefully check that the pressure remained at the correct level and re-adjusted it if needed. The total time blood flow was partially occluded was $\sim 18 \mathrm{~min}$ of the $\sim 25 \mathrm{~min}$ session. Finally, each week participants visited the laboratory to perform one supervised training session. This provided an opportunity to ensure proper movement form, adjust the resistance level if needed, and verify that cuff pressure was being set correctly and carefully monitored. If participants were unable to visit the laboratory, they were instructed to communicate with the investigator via phone or email.

\section{Muscle Soreness}

Before each training session ACLR participants performed a bodyweight halfsquat to a knee angle of $45^{\circ}$ to assess the level of perceived muscle soreness in their legs. Participants were asked to place a mark on a $10 \mathrm{~cm}$ visual analog scale. ${ }^{51}$ This scale was anchored by written cues at $0 \mathrm{~cm}$ (no soreness) and $10 \mathrm{~cm}$ (very severe soreness). Muscle soreness was quantified by measuring the distance to the mark.

\section{Limb Occlusion Pressure}


The cuff was wrapped around the thigh of the ACLR leg at the most proximal location while participants were in a seated position. Doppler ultrasound (Logiq e BT12, GE Healthcare, Chicago, IL, USA) equipped with a linear array transducer operating at an imaging frequency of $12 \mathrm{MHz}$ was used to image the superficial femoral artery. The ultrasound probe was positioned distal to the cuff. The cuff was inflated to $75 \mathrm{mmHg}$ and pressure was increased until blood flow velocity reached zero. The minimum pressure required to completely occlude blood flow was recorded as limb occlusion pressure.16

\section{Quadriceps Size and Strength}

Thickness of the rectus femoris and vastus lateralis were measured separately in the ACLR and uninvolved legs (ACLR group) and nondominant and dominant legs (uninjured control group) using B-mode ultrasound (Logiq e BT12, GE Healthcare, Chicago, IL, USA). Participants were positioned supine with knees resting comfortably in extension with a knee angle of $10^{\circ}$ and a rolled towel was placed under the knee for support. The scanning probe was used to image the muscle. Images of the rectus femoris and vastus lateralis were taken at $66 \%$ of the distance from the anterior superior iliac spine to the proximal patella and thickness was measured as the widest distance between the superficial and deep aponeurosis.29 Muscle thickness measurements were taken $5 \mathrm{x} /$ day on two separate days and the average of the 10 measurements was used for analysis. If the average from each day differed by more than $2.5 \%$ then muscle thickness measurements were taken on an additional third day. The same investigator performed all the ultrasound measurements. Muscle thickness values in each leg were used to calculate a symmetry index (described below). 
Isotonic single-leg knee extensor strength was evaluated on a knee extension strength machine (Cybex, Life Fitness, Rosemont, IL, USA). Participants completed 10 repetitions of weight at $\sim 50 \%$ of their anticipated $1 \mathrm{RM}$ as a warm-up. Participants were then given a $2 \mathrm{~min}$ rest and asked to complete 10 repetitions at $\sim 80 \%$ of their anticipated 1RM. If the participant successfully completed 10 repetitions with proper form, 5-10lbs were added and the participant attempted another 10 repetitions following a 2 min rest. This was repeated until the participant could no longer perform 10 repetitions with proper form. The investigator closely monitored the movement to ensure that full range of motion was achieved and that compensatory efforts were minimized. The weight in pounds and number of repetitions completed were recorded and 1RM was estimated using the Brzycki4 equation: $1 \mathrm{RM}=\mathrm{w}^{*}[36 /(37-\mathrm{r})]$, where $\mathrm{w}$ is the weight in pounds and $r$ is the number of repetitions. Strength values were used to calculate symmetry index.

\section{Symmetry Index}

For the ACLR group, symmetry index was calculated as: $\mathrm{SI}=[(\mathrm{ACLR}$ leg/uninvolved leg)*100]. Accordingly, a number greater than $100 \%$ indicated that the ACLR leg had a greater value than the uninvolved leg. For the control group, the symmetry index was calculated as: $\mathrm{SI}=[($ nondominant leg $/($ dominant leg $) * 100]$. Thus, a number greater than $100 \%$ indicated that the nondominant leg had a greater value than the dominant leg.

\section{Statistical Analysis}


Independent t-tests were used to compare differences in demographic characteristics between the ACLR and uninjured control groups. Additionally, independent t-tests were used to assess differences in baseline rectus femoris thickness, vastus lateralis thickness, and knee extensor strength symmetry indices between the ACLR and uninjured control groups. For the ACLR group, student's paired t-tests were used to assess differences in baseline rectus femoris thickness, vastus lateralis thickness, and knee extensor strength in the ACLR and uninvolved legs. A one-way repeated measures analysis of variance (ANOVA) was performed on muscle soreness values. Separate 2 (ACLR vs. uninvolved leg) x 2 (baseline vs. post-training) repeated measures ANOVA procedures were used to assess changes in rectus femoris thickness, vastus lateralis thickness, and knee extensor strength. Subsequent follow-up paired t-tests were used to test for simple main effects. Paired t-tests were also used to assess differences in baseline and post-training symmetry indices for the ACLR group. Finally, independent ttests were used to evaluate differences in symmetry indices between ACLR (posttraining) and uninjured control groups (baseline). Alpha was set to 0.05 and data were presented as mean $\pm \mathrm{SD}$.

\section{B.1.4 Results}

\section{Baseline}

There were no differences in age, height, body mass, BMI, or body fat between the ACLR and uninjured control groups (all P $>0.05$; Table 1). Participants in the ACLR group had surgery $5 \pm 2$ yrs ago with an autograft of either the semitendinosus muscle tendon $(n=3)$ or patellar tendon $(n=6)$. For these participants, rectus femoris thickness, 
vastus lateralis muscle thickness, and knee extensor strength were reduced in the ACLR leg compared to the uninvolved leg (all $\mathrm{P}<0.01$; Figure 2, 3). As expected mean symmetry values for rectus femoris thickness ( $87 \pm 5)$, vastus lateralis thickness $(90 \pm 6)$, and knee extensor strength $(88 \pm 4)$ were all $\leq 90 \%$ and thus were within the range considered as moderate asymmetry. 34 These symmetry indices for the ACLR group were less than those for the uninjured control group (all $\mathrm{P}<0.01$; Figure 2, 3).

\section{Exercise Training}

Mean limb occlusion pressure was $182 \pm 28 \mathrm{mmHg}$. Accordingly, mean BFR training pressure was set at $91 \pm 14 \mathrm{mmHg}$. ACLR participants completed $98 \%$ of the total exercise sessions and there were no adverse events with the exercise program. Mean muscle soreness $(0-10 \mathrm{~cm}$ analog scale) with the exercise training was very low and did not differ across the training sessions $(0.41 \pm 0.44$ to $0.91 \pm 1.31 \mathrm{~cm}$; $\mathrm{P}>0.05$; Figure 4$)$.

\section{Post-Training}

The repeated measures ANOVA revealed a significant leg $\mathrm{x}$ time interaction for rectus femoris muscle thickness $(\mathrm{P}<0.01)$ indicating that the ACLR leg exhibited a greater change in thickness compared to the uninvolved leg (Figure 2). Further, after training rectus femoris thickness increased by $11 \pm 5 \%(\mathrm{P}<0.01)$ in the ACLR leg but did not change in the uninvolved leg $(\mathrm{P}=0.76)$. A small but significant difference was also present in rectus femoris thickness between the ACLR and uninvolved legs after training $(\mathrm{P}=0.03)$. Post-training rectus femoris thickness symmetry in the ACLR group increased compared to baseline $(\mathrm{P}<0.01)$ and was not different from that for the uninjured control 
group ( $\mathrm{P}=0.28$; Figure 2$)$. The ACLR leg also exhibited a greater change in vastus lateralis thickness compared to the uninvolved leg ( $\mathrm{P}<0.01$; Figure 2). Specifically, after training vastus lateralis thickness increased by $10 \pm 6 \%$ in the ACLR leg $(\mathrm{P}<0.01)$ but did not change in the uninvolved leg $(\mathrm{P}=0.47)$. There was, however, a small but significant difference in vastus lateralis thickness between the ACLR and uninvolved legs after training $(\mathrm{P}=0.02)$. Post-training symmetry for vastus lateralis thickness in the ACLR group increased compared to baseline (both $\mathrm{P}<0.01$ ) but was still lower than that for the uninjured control group $(\mathrm{P}=0.03$; Figure 2).

There was a significant leg $\mathrm{x}$ time interaction for knee extensor strength $(\mathrm{P}<0.01)$ indicating that the ACLR leg exhibited a greater change in strength compared to the uninvolved leg (Figure 2). After training, knee extensor strength increased by $20 \pm 14 \%$ in ACLR leg $(\mathrm{P}<0.01)$ and did not change in the uninvolved leg $(\mathrm{P}=0.09)$. Following training, knee extensor strength in the ACLR and uninvolved legs did not differ $(\mathrm{P}=0.71)$. Accordingly, post-training knee extensor strength symmetry in the ACLR group increased compared to baseline $(\mathrm{P}<0.01)$ and did not differ from that for the uninjured control group $(\mathrm{P}=0.95$; Figure 3$)$.

\section{B.1.5 Discussion}

\section{Main Findings}

In this investigation, participants had an ACLR on average 5yrs ago and despite completing a post-operative rehabilitation program and being physically active still had persistent quadriceps impairments and moderate levels of asymmetry. Accordingly, we 
implemented a 4 week home-based BFR program consisting of bodyweight and walking exercises to increase quadriceps size and strength. As expected, exercise with BFR increased rectus femoris and vastus lateralis thickness as well as knee extensor strength to a greater extent in the ACLR leg compared to the uninvolved leg. Consequently, asymmetry in muscle thickness and knee extensor strength measures was reduced such that symmetry levels (96-99\%) were much closer to those of uninjured controls (99$101 \%)$. Collectively, these findings support our hypotheses, shed new light on the application of home-based BFR exercise, and offer a promising approach for restoration of quadriceps function long after ACLR.

\section{Home-Based Exercise with BFR}

A unique aspect of this study was the development of a home-based BFR exercise protocol. To minimize the need for expensive exercise equipment we implemented simple exercises that only required the use of a thigh blood pressure cuff and a resistance band ( $\$ 20$ USD). Further, when implementing BFR exercise, selection of cuff pressure is especially critical as this affects not only the training stimulus for improvement but also the safety of the participant. Indeed, cuff pressure is influenced by several factors including cuff size, limb anthropometrics, sex, and race and thus is highly variable across individuals. ${ }^{17}$ With this in mind, we used moderate training pressures $(\sim 90 \mathrm{mmHg})$ that were normalized to $50 \%$ of each participant's limb occlusion pressure. Using this approach, participants were able to perform BFR exercise without causing injury and tolerated the training as muscle soreness was very low $(<1.0 \mathrm{~cm}, 0$ - no soreness to 10 - 
very severe soreness). Thus, implementation of BFR exercise at home provided a program that was feasible, safe, and inexpensive.

\section{Quadriceps Size and Strength}

Restoration of quadriceps size and strength is imperative for recovery of knee joint function following ACLR and long-term joint health. ${ }^{35}$ Achievement of $90 \%$ symmetry $y^{5,23,34,39}$ is often used as one of the clinical milestones for returning to physical activity and sport. Our inclusion criteria included active individuals who had $<90 \%$ symmetry and the moderate level of asymmetry present in these individuals before training was not surprising as several authors ${ }^{20,21,26}$ have reported persistent asymmetry for many years following ACLR. After 4 weeks of BFR exercise, rectus femoris and vastus lateralis thickness in the ACLR leg increased by $11 \%$ and $10 \%$, respectively. Increased quadriceps thickness is significant because knee extensor strength is more associated with quadriceps volume and cross sectional area rather than voluntary activation as individuals with knee surgery pass the $\sim 1$ yr mark. ${ }^{20,30,36}$ The BFR home program also increased knee extensor strength by $20 \%(\sim 6.5 \mathrm{~kg})$. Together, these BFR exercise-induced improvements in quadriceps muscle thickness and knee extensor 1RM strength are consistent with those for healthy adults performing BFR resistance 2 and aerobic1 exercise where quadriceps volume and whole-leg 1RM strength increased by $\sim 6 \%$ and $\sim 12 \%$, respectively. The results of the current study also provide additional evidence that BFR exercise-induced muscle and strength gains can be achieved quickly and through both resistance and aerobic exercise. ${ }^{27,41}$ 
With increased muscle thickness and strength in the ACLR leg there was a notable improvement in symmetry for rectus femoris (87 to $96 \%)$ and vastus lateralis (90 to $97 \%$ ) thickness and knee extensor strength (88 to $99 \%$ ). Indeed, post-training symmetry values were much closer to those of uninjured controls. Most importantly, these participants living with ACLR were able to overcome presumably years of quadriceps weakness with just one month of targeted exercise training and achieve remarkable levels of knee extensor strength symmetry. These symmetry outcomes extend upon previous reports documenting the application of BFR exercise early after ACLR. For example, Ohta and colleagues ${ }^{32}$ reported that BFR exercise implemented 3-16 weeks post-operatively increased muscle cross-sectional area (91 to 101\%) and knee extensor strength (65 to $77 \%$ ) symmetry. Taken together, the results from the current study along with previous reports ${ }^{22,32,44}$ clearly demonstrate that BFR exercise can offer a potent stimulus for improving quadriceps function after ACLR. Moreover, implementation of exercise involving relatively low loads to improve quadriceps function in individuals that have a history of ACLR is especially noteworthy because traditional high-load resistance exercise can be injurious and intolerable.

The physiological mechanisms responsible for increased muscle size and strength with BFR exercise are not well established. Previous authors have proposed a variety of mechanisms including accelerated fatigue leading to preferential type II fiber recruitment, ${ }^{52}$ augmented metabolite accumulation and local hypoxia, ${ }^{40}$ and cell swelling. ${ }^{24}$ There may also be an endocrine response as exercise with BFR increases serum growth hormone to a greater extent than traditional high load resistance exercise. ${ }^{37}$ 
This increase in growth hormone secretion possibly occurs through the stimulation of chemoreceptors due to decreases in muscle perfusion. ${ }^{45}$ Determination of the exact mechanisms goes beyond the scope of the present data and thus our results reinforce the notion that BFR exercise offers an effective intervention for improving muscular function in healthy and clinical populations.

\section{Implications and Considerations}

Our findings have several important implications for clinicians. First, when patients with ACLR are eventually discharged from supervised rehabilitation, clinicians may consider incorporating BFR exercises into home-based maintenance programs. Specifically, this may allow these individuals to further increase their quadriceps size and strength, symmetry, and/or physical function because low-load BFR resistance exercise is more effective than traditional low-load resistance exercise. ${ }^{25}$ An alternative possibility is that low-load BFR resistance exercise may enable individuals to progress sooner towards high-load resistance exercise, ${ }^{15}$ which elicits even greater strength gains. Increased dynamic stability through the knee as a result of increased quadriceps strength might decrease the risk of secondary, long-term osteoarthritic changes. ${ }^{49}$ Second, individuals with ACLR that have persistent quadriceps impairments and require follow-up medical intervention may find BFR exercise practical as it can be performed at home, facilitates strength gains quickly, and involves inexpensive equipment. Finally, given that BFR exercise can be administered effectively early after knee arthoscopy ${ }^{46}$ and total knee 
arthoplasty ${ }^{10}$ procedures, it would very interesting to determine if implementation of BFR exercise long after these procedures can also help these individuals overcome persistent quadriceps impairments.

It is important to acknowledge that BFR exercise is characterized by slight discomfort and dull pain at the site of the cuff which could affect an individual's motivation and program compliance. In addition, restriction of blood flow during exercise does elevate heart rate and blood pressure and, although unlikely, could give rise to severe side effects (e.g., adverse cardiovascular responses, blood clotting, muscle/nerve damage). ${ }^{43}$ To date, many investigators have implemented BFR exercise safely across a broad range of healthy, ${ }^{27,41}$ athletic, ${ }^{42}$ and clinical ${ }^{15}$ populations. Adverse effects due to BFR are rare. ${ }^{31}$ There are no standardized guidelines for selection of cuff pressure. Current best practices indicate that cuff pressure be based on individual limb occlusion pressure or thigh circumference. ${ }^{41} \mathrm{With}$ these considerations in mind, careful selection of cuff pressure, continued monitoring of training, and participant education is critical to ensuring that BFR exercise be implemented safely.

\section{Limitations}

In this investigation, participants represented a small sample from the general ACLR population. This ACLR cohort was young and physically active and thus the results may not be entirely transferrable to older and/or less active individuals dealing with persistent quadriceps impairments. This study also focused on restoring quadriceps

size, strength, and symmetry. While isolated quadriceps size and strength do contribute to 
improved physical function there are a number of additional factors that warrant consideration such as quadriceps muscle activation, ${ }^{12}$ muscle co-contraction, ${ }^{8,50}$ and hip and trunk strength. ${ }^{38}$ Further, because changes in physical function were not directly assessed in the current study it is unknown if the increased muscle thickness, knee extensor strength, and symmetry actually translated to better physical function and performance. Future work that includes a larger sample along with a combination of structural, functional, and subjective measures is needed to understand the benefits and limitations of using BFR exercise long after ACLR.

\section{Summary}

This is the first study to report the use of home-based BFR exercise to increase quadriceps size and strength several years after ACLR. Specifically, ${ }^{4}$ weeks of BFR exercise resulted in significant increases in rectus femoris and vastus lateralis thickness and dynamic knee extensor strength while inducing very little muscle soreness. Moreover, BFR exercise was sufficient in restoring knee extensor strength symmetry to that of uninjured controls. We conclude that home-based BFR exercise offers a safe and effective intervention to improve quadriceps function long after ACLR. These results support, unite, and extend upon previous BFR exercise studies and have implications for clinicans who treat individauls that have persistent quadriceps impariments.

\section{KEY POINTS}

FINDINGS: Implementation of BFR exercise several years after ACLR increased quadriceps muscle thickness and knee extensor strength and reduced asymmetry. In 
addition, the home-based program consisting of bodyweight and walking exercises with BFR was feasible, safe, and inexpensive.

IMPLICATIONS: BFR exercise offers a promising approach for restoration of quadriceps function and symmetry long after ACLR.

CAUTION: To implement BFR exercise safely and effectively, careful selection of cuff pressure, continued monitoring of training, and participant education is needed.

\section{REFERENCES}

1. Abe T, Kearns CF, Sato Y. Muscle size and strength are increased following walk training with restricted venous blood flow from the leg muscle, Kaatsu-walk training. J Appl Physiol (1985). 2006;100(5):1460-1466.

2. Abe T, Yasuda T, Midorikawa T, et al. Skeletal muscle size and circulating IGF-1 are ncreased after two weeks of twice daily "KAATSU" resistance training. Int J KAATSU Training Research. 2005;1(1):6-12.

3. Ardern CL, Webster KE, Taylor NF, Feller JA. Return to sport following anterior cruciate ligament reconstruction surgery: a systematic review and meta-analysis of the state of play. Br J Sports Med. 2011;45:596-606. 
4. Brzycki M. Strength testing: predicting one-rep max from repetitions to fatigue. J Phys Edu Recr Dan. 1993;64(1):88-90.

5. Curran MT, Lepley LK, Palmieri-Smith RM. Continued improvements in quadriceps strength and biomechanical symmetry of the knee after postoperative anterior cruciate ligament reconstruction rehabilitation: Is it time to reconsider the 6-month return-to-activity criteria? Athl Train. 2018;53:535-544.

6. Di Stasi SL, Logerstedt D, Gardinier ES, Snyder-Mackler L. Gait patterns differ between ACL-reconstructed athletes who pass return-to-sport criteria and those who fail. Am J Sports Med. 2013;41(6):1310-1318.

7. de Jong SN, van Caspel DR, van Haeff MJ, Saris DB. Functional assessment and muscle strength before and after reconstruction of chronic anterior cruciate ligament lesions. Arthroscopy. 2007;23(1):21-8, 28.e1-3.

8. Elias AR, Hammill CD, Mizner RL. Changes in quadriceps and hamstring cocontraction following landing instruction in patients with anterior cruciate ligament reconstruction. J Orthop Sports Phys Ther. 2015;45(4):273-280.

9. Garber CE, Blissmer B, Deschenes MR, Franklin BA, Lamonte MJ, Lee IM, Nieman DC, Swain DP. American College of Sports M. American College of Sports Medicine position stand. Quantity and quality of exercise for developing and maintaining cardiorespiratory, musculoskeletal, and neuromotor fitness in apparently healthy adults: guidance for prescribing exercise. Med Sci Sports Exerc. 2011. 43:1334-1359. 
10. Gaunder CL, Hawkinson MP, Tennent DJ, Tubb CC. Occlusion training: pilot study for postoperative lower extremity rehabilitation following primary total knee arthroplasty. US Army Med Dep J. 2017:39-43.

11. Grant JA, Mohtadi NG, Maitland ME, Zernicke RF. Comparison of home versus physical therapy-supervised rehabilitation programs after anterior cruciate ligament reconstruction: a randomized clinical trial. Am J Sports Med. 2005;33(9):1288-1297.

12. Hart JM, Pietrosimone B, Hertel J, Ingersoll CD. Quadriceps Activation Following Knee Injuries: A Systematic Review. J Athl Train. 2010;45(1): 87-97.

13. Henning CE, Lynch MA, Glick KR, Jr. An in vivo strain gage study of elongation of the anterior cruciate ligament. Am J Sports Med. 1985;13(1):22-26.

14. Hiemstra LA, Webber S, MacDonald PB, Kriellaars DJ. Knee strength deficits after hamstring tendon and patellar tendon anterior cruciate ligament reconstruction. Med Sci Sports Exerc. 2000;32(8):1472-1479.

15. Hughes L., Paton B., Rosenblatt B., Gissane C., Patterson S.D. Blood flow restriction training in clinical musculoskeletal rehabilitation: a systematic review and meta-analysis. Br J Sports Med. 2017;51:1003-1011.

16. Hunt JE, Stodart C, Ferguson RA.The influence of participant characteristics on the relationship between cuff pressure and level of blood flow restriction. Eur J Appl Physiol. 2016;116(7):1421-32. 
17. Jessee MB, Buckner SL, Dankel SJ, Counts BR, Abe T, Loenneke JP. The influence of cuff width, sex, and race on arterial occlusion: Implications for blood flow restriction research. Sports Med. 2016;46:913-21.

18. Keays SL, Bullock-Saxton J, Newcombe P, Keays AC. The relationship between knee strength and functional stability before and after anterior cruciate ligament reconstruction. J Orthop Res. 2003;21:231-237.

19. Konishi Y, Ikeda K, Nishino A, Sunaga M, Aihara Y, Fukubayashi T. Relationship between quadriceps femoris muscle volume and muscle torque after anterior cruciate ligament repair. Scand J Med Sci Sports. 2007;17(6):656-661.

20. Krishnan C, Williams GN. Factors explaining chronic knee extensor strength deficits after ACL reconstruction. J Orthop Res. 2011;29(5):633-40.

21. Kuenze CM, Hertel J, Weltman A, Diduch D, Saliba SA, Hart JM. Persistent neuromuscular and corticomotor quadriceps asymmetry after anterior cruciate ligament reconstruction. J Athl Train. 2015;50(3):303-12.

22. Lejkowski PM, Pajaczkowski JA. Utilization of Vascular Restriction Training in post-surgical knee rehabilitation: a case report and introduction to an under-reported training technique. J Can Chiropr Assoc. 2011; 55:280-287

23. Lewek M, Rudolph K, Axe M, Snyder-Mackler L. The effect of insufficient quadriceps strength on gait after anterior cruciate ligament reconstruction. Clin Biomech (Bristol, Avon). 2002;17:56-63. 
24. Loenneke JP, Fahs CA, Rossow LM, Abe T, Bemben MG. The anabolic benefits of venous blood flow restriction training may be induced by muscle cell swelling. Med Hypotheses. 2012;78(1):151-154.

25. Loenneke JP, Wilson JM, Marin PJ, Zourdos MC, Bemben MG. Low intensity blood flow restriction training: a meta-analysis. Eur J Appl Physiol. 2012;112(5):18491859.

26. Longo UG, Rizzello G, Frnaceschi F, Campi S, Maffulli N, Denaro V. The architecture of the ipsilateral quadriceps two years after successful anterior cruciate ligament reconstruction with bone-patellar tendon-bone autograft. Knee. 2014;21(3):721725.

27. Manini TM, Clark BC. Blood flow restricted exercise and skeletal muscle health. Exerc Sport Sci Rev. 2009;37:78-85.

28. Marcon M, Ciritsis B, Laux C, et al. Quantitative and qualitative MR-imaging assessment of vastus medialis muscle volume loss in asymptomatic patients after anterior cruciate ligament reconstruction. J Magn Reson Imaging. 2015;42:515-525.

29. Martin-Hernandez J, Marin PJ, Menendez H, et al. Changes in muscle architecture induced by low load blood flow restricted training. Acta Physiol Hung. 2013;100:411-418. 
30. Meier WA, Marcus RL, Dibble LE et al. The long-term contribution of muscle activation and muscle size to quadriceps weakness following total knee arthro-plasty. J Geriatr Phys Ther. 2009;32(2):35-38.13.

31. Nakajima T, Kurano M, Iida H, et al. Use and safety of KAATSU training: results of a national survey. Int J KAATSU Training Research. 2006;2(1):5-13.

32. Ohta H, Kurosawa H, Ikeda H, Iwase Y, Satou N, Nakamura S Low-load resistance muscular training with moderate restriction of blood flow after anterior cruciate ligament reconstruction. Acta Orthop Scand. 2003;74:62-68.

33. Oiestad BE, Holm I, Gunderson R, et al. Quadriceps muscle weakness after anterior cruciate ligament reconstruction: a risk factor for knee osteoarthritis? Arthritis Care Res (Hoboken). 2010;62:1706-1714.

34. Palmieri-Smith RM, Lepley LK. Quadriceps Strength Asymmetry After Anterior Cruciate Ligament Reconstruction Alters Knee Joint Biomechanics and Functional Performance at Time of Return to Activity. Am J Sports Med. 20151;43(7):1662-9.

35. Palmieri-Smith RM, Thomas AC, Wojtys EM. Maximizing quadriceps strength after ACL reconstruction. Clin Sports Med. 2008;27: 405-424, vii-ix.

36. Petterson S, Barrance P, Marmon A et al. Time course of quad strength, area andactivation after knee arthroplasty and strength training. Med Sci Sports Exerc. 2011;43(2):225-23. 
37. Pierce JR, Clark BC, Ploutz-Snyder LL, Kanaley JA. Growth hormone and muscle function responses to skeletal muscle ischemia. J Appl Physiol (1985). 2006;101(6):1588-1595.

38. Powers CM. The influence of abnormal hip me $\neg$ chanics on knee injury: a biomechanical perspec $\neg$ tive. J Orthop Sports Phys Ther. 2010;40:42-51.

39. Schmitt LC, Paterno MV, Hewett TE. The impact of quadriceps femoris strength asymmetry on functional performance at return to sport following anterior cruciate ligament reconstruction. J Orthop Sports Phys Ther. 2012;42(9):750-759.

40. Scott BR, Slattery KM, Sculley DV, Dascombe BJ. Hypoxia and resistance exercise: a comparison of localized and systemic methods. Sports Med. 2014;44(8):10371054.

41. Scott BR, Loenneke JP, Slattery KM., Dascombe B.J. Exercise with blood flow restriction: an updated evidence-based approach for enhanced muscular development. Sports Med. 2015;45:313-325.

42. Scott BR, Loenneke JP, Slattery KM, Dascombe BJ. Blood flow restricted exercise for athletes: A review of available evidence. J Sci Med Sport. 2016;19:360-367. 
43. Spranger MD, Krishnan AC, Levy PD, O'Leary DS, Smith SA. Blood flow restriction training and the exercise pressor reflex: a call for concern. Am J Physiol Heart Circ Physiol. 2015;309(9):1440-52.

44. Takarada Y, Takazawa H, Ishii N. Applications of vascular occlusion diminish disuse atrophy of knee extensor muscles. Med Sci Sports Exerc. 2000;32:2035-2039.

45. Takarada Y, Nakamura Y, Aruga S, Onda T, Miyazaki S, Ishii N. Rapid increase in plasma growth hormone after low-intensity resistance exercise with vascular occlusion. J Appl Physiol (1985). 2000;88(1):61-65.

46. Tennent DJ, Hylden CM, Johnson AE, Burns TC, Wilken JM, Owens JG. Blood Flow Restriction Training After Knee Arthroscopy: A Randomized Controlled Pilot Study. Clin J Sport Med. 2017;27(3):245-252.

47. Thomas AC, Villwock M, Wojtys EM, et al. Lower extremity muscle strength after anterior cruciate ligament injury and reconstruction. J Athl Train. 2013;48:610-620.

48. Thomas AC, Wojtys EM, Brandon C, Palmieri-Smith RM. Muscle atrophy contributes to quadriceps weakness after anterior cruciate ligament reconstruction. J Sci Med Sport. 2016;19:7-11.

49. Tourville TW, Jarrell KM, Naud S, Slauterbeck JR, Johnson RJ, Beynnon BD. Relationship between isokinetic strength and tibiofemoral joint space width changes after anterior cruciate ligament reconstruction. Am J Sports Med. 2014;42(2):302-311. 
50. Tsai LC, McLean S, Colletti PM, Powers CM. Greater muscle cocontraction results in increased tibiofemoral compressive forces in females who have undergone anterior cruciate ligament reconstruction. J Orthop Res. 2012;30(12):2007-2014.

51. Twist C, Eston R. The effects of exercise-induced muscle damage on maximal intensity intermittent exercise performance. Eur J Appl Physiol. 2005;94(5-6):652-658.

52. Yasuda T, Brechue WF, Fujita T, Shirakawa J, Sato Y, Abe T. Muscle activation during low-intensity muscle contractions with restricted blood flow. J Sports Sci. 2009;27(5):479-489. 
TABLE 1. Participant demographics

\begin{tabular}{|c|c|c|c|}
\hline Variable & $\operatorname{ACLR}(n=9)$ & $\begin{array}{c}\text { Uninjured Control } \\
(n=9)\end{array}$ & $\begin{array}{c}P \\
\text { value }\end{array}$ \\
\hline Age (yrs) & $26 \pm 8$ & $26 \pm 6$ & 0.81 \\
\hline Height (m) & $1.74 \pm 0.14$ & $1.73 \pm 0.08$ & 0.88 \\
\hline Body mass (kg) & $73 \pm 16$ & $71 \pm 12$ & 0.70 \\
\hline BMI $\left(\mathrm{kg} \cdot \mathrm{m}^{-2}\right)$ & $24 \pm 2$ & $24 \pm 3$ & 0.67 \\
\hline Body fat (\%) & $29 \pm 7$ & $27 \pm 5$ & 0.61 \\
\hline \multicolumn{4}{|l|}{ Sex } \\
\hline Male & 3 & 3 & \\
\hline Female & 6 & 6 & \\
\hline $\begin{array}{l}\text { Time from ACLR } \\
\text { (yrs) }\end{array}$ & $5 \pm 2$ & ----- & \\
\hline \multicolumn{4}{|l|}{ Graft type } \\
\hline $\begin{array}{l}\text { Hamstring } \\
\text { tendon }\end{array}$ & 3 & ----- & \\
\hline Patellar tendon & 6 & ---- & \\
\hline
\end{tabular}

Values are reported as Mean \pm SD. Note that, body fat data are reported on $n=7$ for the ACLR group and $n=8$ uninjured control group. 


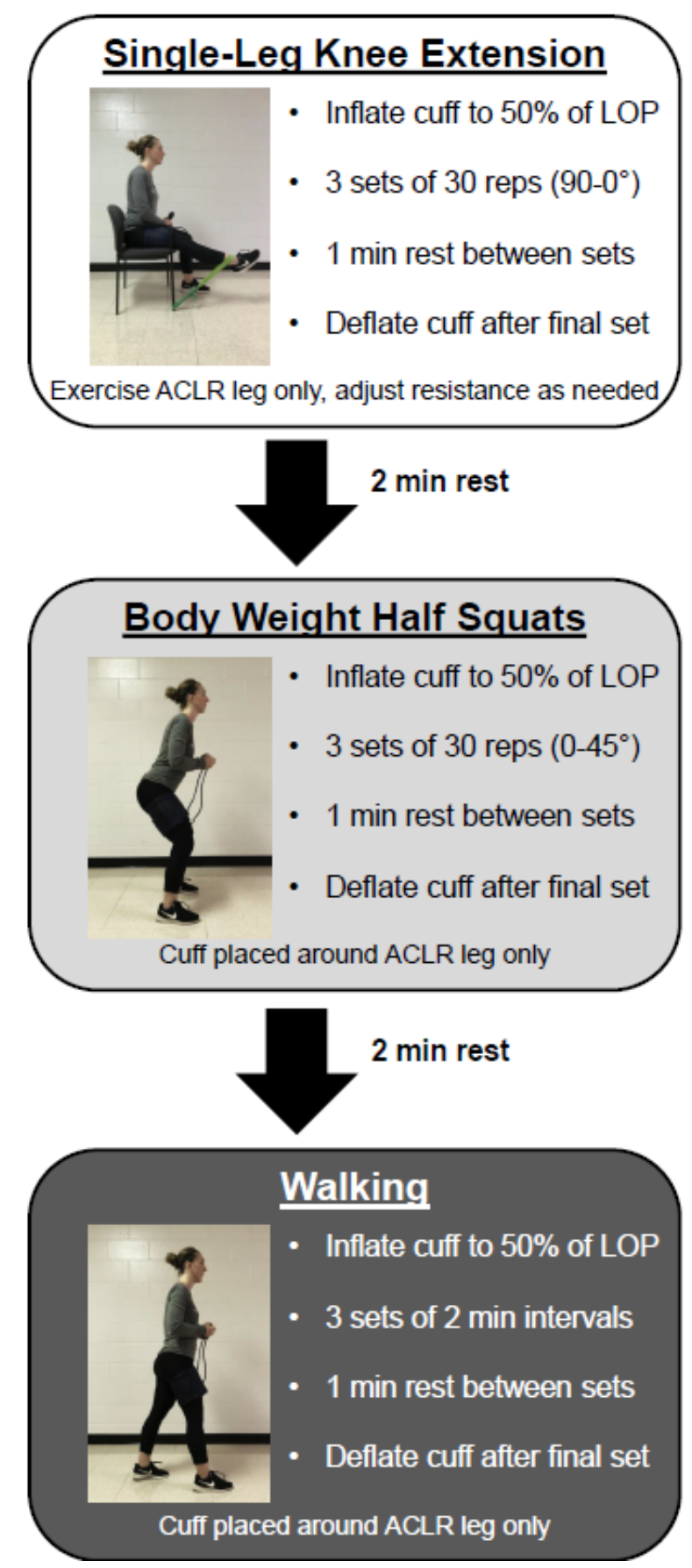

Figure 1. Overview a home-based BFR exercise training session. Each training session consisted of a series of three exercises (single-leg knee extension, body weight half squats, walking) and took $\sim 25 \mathrm{~min}$ to complete. During each exercise, blood flow to the working muscles in the ACLR leg was restricted using a thigh cuff set to $50 \%$ of limb occlusion pressure (LOP). Participants performed 5 training sessions/week for 4 weeks. 

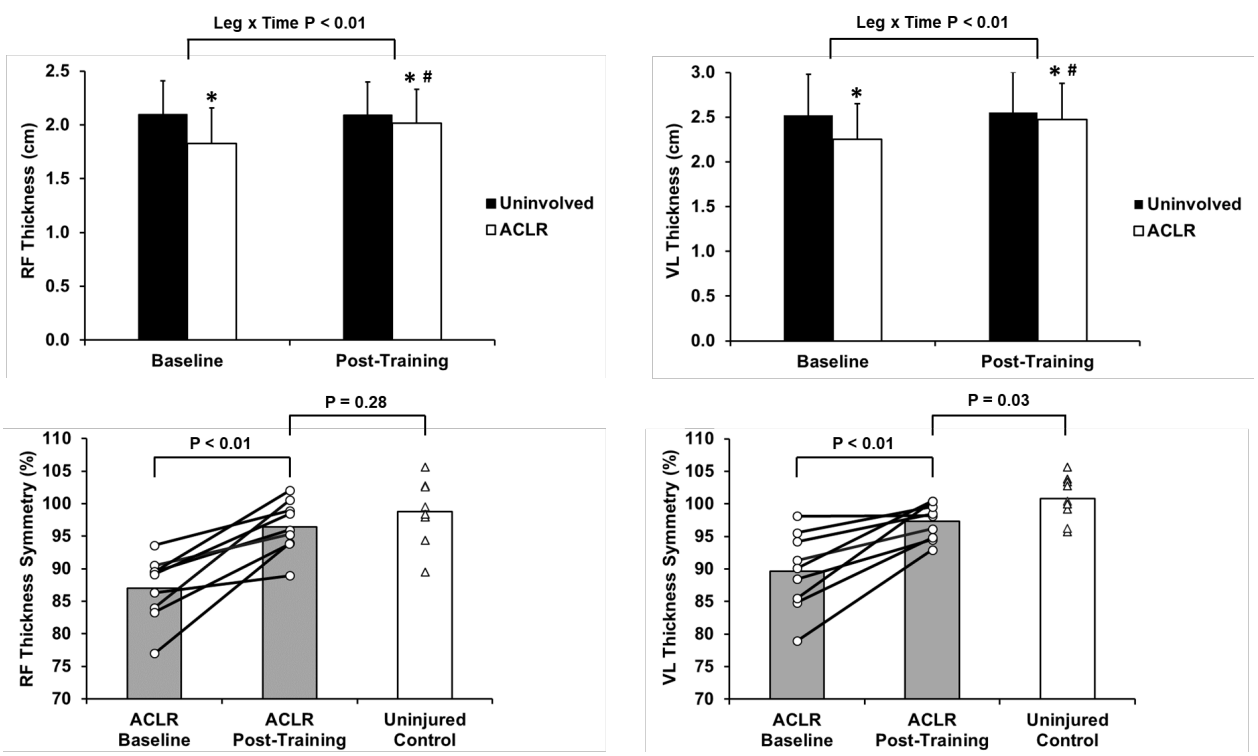

Figure 2: Alterations in rectus femoris (RF) and vastus lateralis (VL) muscle thickness in the ACLR and uninvolved leg for the ACLR group (top panels). Muscle thickness symmetry in the ACLR and uninjured control groups (bottom panels). For the ACLR group a number \&lt; $100 \%$ indicated uninvolved leg dominance. Solid lines represent individual changes from baseline to post-training for the ACLR group and open triangles represent individual values for the uninjured control group. Data are reported as mean \pm SD. * 0.05 vs uninvolved leg. \# 0.05 vs. baseline. 


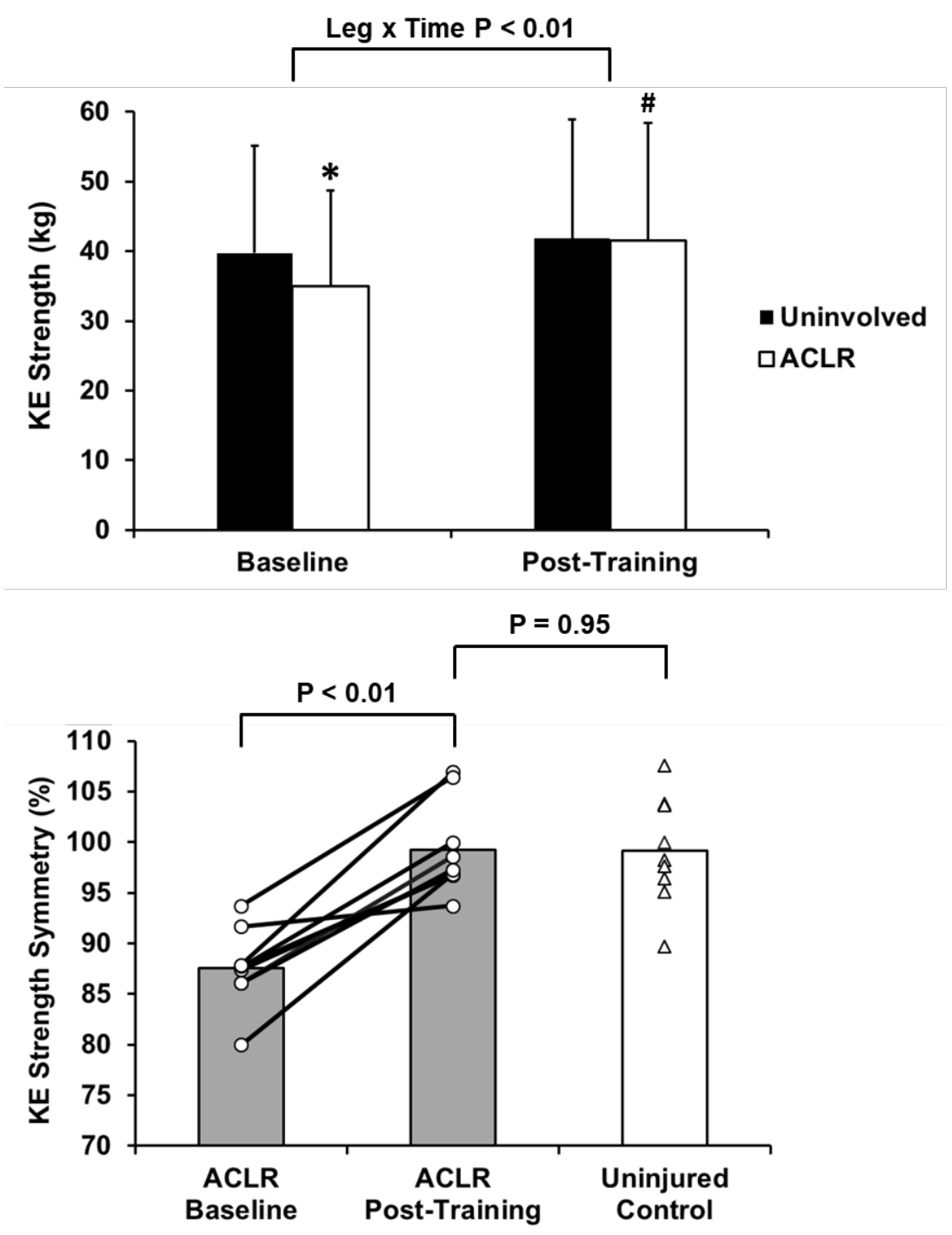

FIGURE 3. Changes in knee extensor 1RM strength in the ACLR and uninvolved leg for the ACLR group (top panel). Knee extensor 1RM strength symmetry in the ACLR and uninjured control groups (bottom panel). For the ACLR group a number $100 \%$ indicated uninvolved leg dominance. Solid lines represent individual changes from baseline to post-training for the ACLR group and open triangles represent individual values for the uninjured control group. Data are reported as mean \pm SD. * 0.05 vs uninvolved leg. \# 0.05 vs. baseline. 


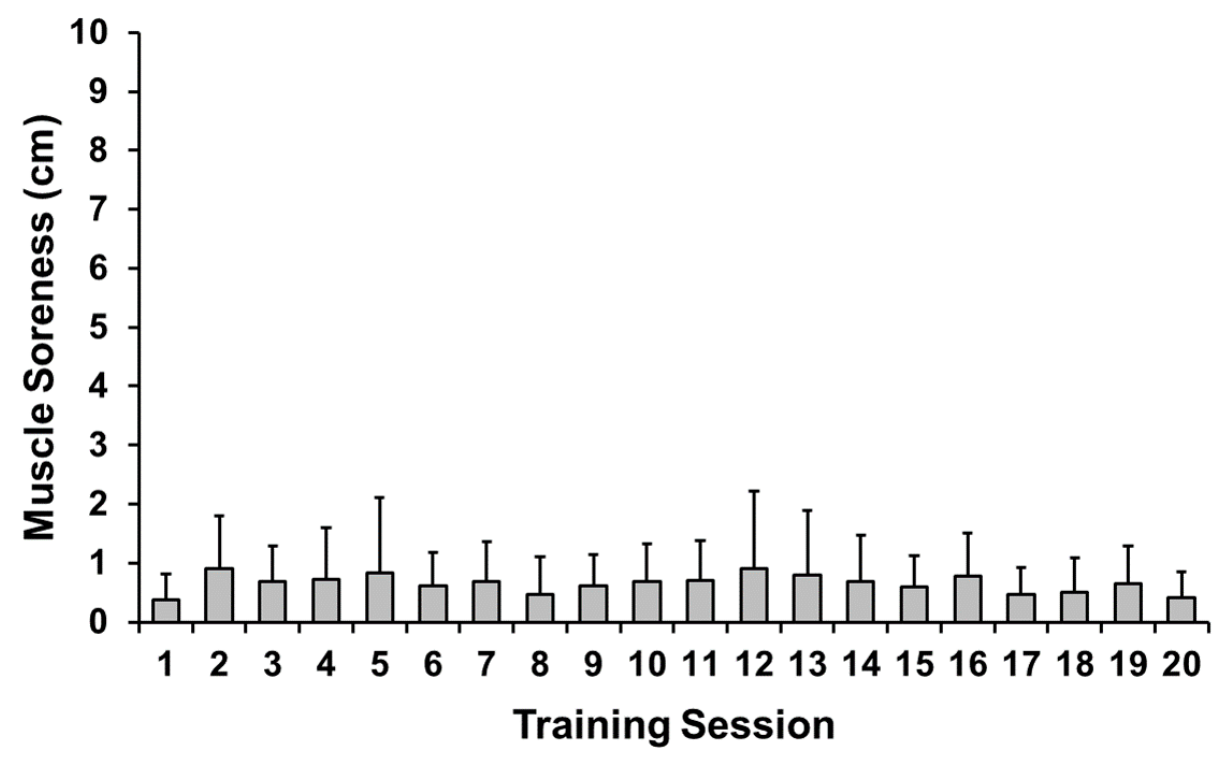

FIGURE 4. Perceived muscle soreness associated with the home-based BFR exercise program (0 - no soreness, 10 - severe soreness). Data are reported as mean $\pm \mathrm{SD}$. 


\section{B.2 Home-Based Exercise with Blood Flow Restriction to Improve Quadriceps and Physical Function after Total Knee Arthroplasty: A Case Report}

\section{B.2.1 Abstract}

Background and Purpose: After total knee arthroplasty (TKA) persistent quadriceps muscle atrophy and weakness impairs physical function. Blood flow restriction (BFR) exercise is emerging as an effective method to improve muscle size and strength in clinical populations with orthopedic limitations. There are no randomized controlled studies documenting BFR exercise after TKA. This case report describes the use of home-based BFR exercise to increase quadriceps size, strength, and physical function after TKA.

Case Description: A 59yr old male (6 months post-TKA) performed body weight and walking exercises with BFR 5x/wk for 8wks. Blood flow in the TKA leg was restricted using a thigh cuff inflated to $50 \%$ of limb occlusion pressure. Lean leg mass, vastus lateralis thickness, knee extensor strength, and physical function were measured at baseline (6 months post-TKA), post-training ( 8 months post-TKA), and long-term follow-up (14 months post-TKA).

Outcomes: After training, lean leg mass, vastus lateralis thickness, and knee extensor strength in the TKA leg increased by $4 \%, 14 \%$, and 55\%, respectively. Compared to baseline, post-training knee extensor strength symmetry (TKA/uninvolved leg) increased from $64 \%$ to $98 \%$. The patient's performance improved for the 30 s chair stand, $40 \mathrm{~m}$ fast walk, and $6 \mathrm{~min}$ walk tests. Increased quadriceps and physical function were maintained at the long-term follow-up. 
Discussion: With enhanced quadriceps and physical function the patient resumed independent physical activity. Muscle and strength gains surpassed those typically reported after TKA. Outcomes suggest that home-based BFR exercise was feasible, safe, and effective. BFR exercise after TKA is promising and warrants further research.

\section{B.2.2 Introduction}

Each year more than 650,000 total knee arthroplasty (TKA) surgeries are performed to treat end-stage knee osteoarthritis. ${ }^{1}$ Annual costs associated with TKA are estimated to be $\$ 11$ billion $^{2}$ and will increase dramatically as the number of surgeries is projected to reach 3.5 million by $2030 .{ }^{1}$ After the TKA procedure, knee joint pain is reduced in most individuals. ${ }^{3}$ However, surgery also has a profound impact on quadriceps muscle size and strength. For example, quadriceps strength is substantially reduced after surgery, steadily improves between 3-6 months, and eventually reaches a level that is $\sim 80 \%$ of the uninvolved leg (for a review see reference ${ }^{4}$ ). Persistent quadriceps weakness after TKA is associated with altered gait mechanics5 and reduced physical function6. Moreover, muscle weakness in older adults is a risk factor for falls. ${ }^{7}$ Accordingly, developing effective rehabilitation strategies to restore quadriceps muscle size and strength is imperative so that individuals with TKA can maintain adequate functional mobility and avoid further long-term disability.

Several barriers make it difficult for individuals with TKA to regain their quadriceps size and strength. First, heavy exercise loads (65-70\% of 1-repetition maximum) required to trigger muscle and strength adaptations are not always possible because they may increase pain and/or joint irritation. Second, time requirements for 
complete restoration of quadriceps size and strength can be excessive. Third, access to specialized equipment for enhancing quadriceps muscle strengthening can be limited after discharge to home-based therapy. Implementation of blood flow restriction (BFR) exercise is emerging as a safe and effective method to increase muscle size and strength in clinical populations with orthopedic limitations. ${ }^{8}$ This exercise mode involves lifting low-loads (20-30\% of 1-repetition maximum) while a pressurized cuff reduces blood flow.9 Specifically, cuff pressure is set low enough to partially maintain arterial blood flow to working muscles but high enough to prevent most of the venous blood flow from returning to the heart. Exercise with BFR is advantageous ${ }^{8,9}$ because increases in muscle size and strength are elicited using low-loads, strength gains can be achieved faster than traditional exercise, and it is used with resistance and aerobic exercise. The possibility of using BFR after TKA is intriguing ${ }^{10}$ but there are no randomized controlled studies documenting BFR exercise after TKA. Our purpose was to describe the use of an 8-week home-based BFR exercise program with a patient who had leveled off in his recovery at 6 months post-TKA. We envisioned that BFR exercise would be safely tolerated, increase quadriceps size and strength, and enhance physical function.

\section{B.2.3 Case Description}

\section{Patient}

A $59 \mathrm{yr}$ old male (body mass: $85 \mathrm{~kg}$, height: $1.81 \mathrm{~m}$, BMI: 22 ; body fat: $31 \%$ ) with a history of knee osteoarthritis presented pain and edema in his left knee. The patient developed an antalgic gait, experienced difficulty with reciprocal stair 
climbing/descending, and was unable to participate in his regular physical activity program consisting of walking and cycling 5-6x/wk. A unilateral TKA procedure was performed to alleviate the osteoarthritic pain. The patient completed 2 months of outpatient therapy that focused on range of motion, balance, and quadriceps strengthening. At the time of our evaluation the patient was 6 months post-surgery with full functional knee range of motion and adequate strength for independent ambulation. He had mild knee joint pain ( $1.8 \mathrm{~cm}$ using $0-10 \mathrm{~cm}$ visual analog scale), slight edema, and detectable weakness in the left quadriceps. The patient exhibited an intermittent antalgic gait and difficulty with reciprocal stair climbing/descending. The patient stated that he had "plateaued" in his recovery and needed to achieve higher function in order to resume his physical activity routine.

\section{Examination: Baseline, Post-Training, and Long-Term Follow-Up}

Muscle size, strength, and physical function were evaluated at baseline (6 months post-TKA), post-training ( 8 months post-TKA), and long-term follow-up (14 months post-TKA). Lean leg mass was assessed using dual energy $\mathrm{x}$-ray absorptiometry (Discovery Wi, Hologic Inc, Marlborough, MA, USA). Thickness of the vastus lateralis was assessed using B-mode ultrasound (Logiq e BT12, GE Healthcare, Chicago, IL, USA). Muscle thickness was defined as the distance between the superficial and deep aponeurosis, ${ }^{11}$ assessed $5 \mathrm{x} /$ day on 2 separate days $(\mathrm{COV}=1.7 \%)$, and averaged over the two days. Knee extensor strength was measured during a break test12 using a hand-held dynamometer (Microfet, Hoggan Health Industries, Inc., Murray, UT, USA). The patient performed brief maximal contractions (3-5s) 4x/day on 3 separate days. The average of 
the three closest contractions within each day were used for analysis $(\mathrm{COV}=4.7 \%)$ and knee extensor strength symmetry was calculated as: [(TKA leg-uninvolved leg)/(uninvolved leg)]. ${ }^{13}$ Physical function was assessed using the 30s chair stand, stair climb ( 9 stairs, stair height $18.8 \mathrm{~cm}$ ), $40 \mathrm{~m}$ fast walk, and $6 \mathrm{~min}$ walk tests. Tests were performed 1x/day on two separate days and the average values were used for analysis. These physical function tests are reported to have good reliability. ${ }^{14}$

\section{Intervention}

An overview of the home-based BFR exercise program is illustrated in Figure 1. The patient performed BFR exercise 5x/wk for 8-weeks. Each session consisted of three exercises (single-leg knee extension, body weight half-squats, walking) and took $\sim 25 \mathrm{~min}$ to complete. First, the patient performed 3 sets of 30 single-leg knee extensions using his TKA leg with a Thera-Band ( $\sim 3-51 \mathrm{lbs}$ of resistance). Next, the patient performed 3 sets of 30 double-leg body weight half-squats. Finally, the patient performed 3 sets of $2 \mathrm{~min}$ walking intervals at a self-selected speed. Throughout the session a $1 \mathrm{~min}$ rest period was provided between sets and $2 \mathrm{~min}$ of rest was given between exercises. During each exercise, TKA leg blood flow was restricted using an $18 \mathrm{~cm}$ wide aneroid sphygmomanometer (Briggs, Healthcare, Waukegan, IL, USA). The cuff was wrapped around the proximal part of the thigh and inflated to $108 \mathrm{~mm} \mathrm{Hg}$ which represented $50 \%$ of the pressure required to completely occlude blood flow in the femoral artery $(216 \mathrm{~mm}$ $\mathrm{Hg}$ ). The cuff remained inflated during the $1 \mathrm{~min}$ rest between sets but was deflated during the $2 \mathrm{~min}$ recovery between exercises. Accordingly, blood flow was partially occluded for a total of $\sim 18 \mathrm{~min}$. Each week the patient visited the laboratory to perform 
one supervised session to verify proper movement form and cuff pressure. Before and after each session, the patient recorded any level of quadriceps muscle soreness and knee joint pain (0-10 visual analog scale).

\section{B.2.4 Outcomes}

\section{Program Adherence}

The patient completed 4-weeks of the program, took a 1-week break at Christmas, and finished the final 4-weeks. He completed all 40 total prescribed BFR exercise sessions. Muscle soreness associated with BFR exercise was low $(0.0-1.4 \mathrm{~cm})$. Joint pain persisted throughout the study $(1.2-2.5 \mathrm{~cm})$ but did not limit exercise and was generally lower after exercise $(0.2-1.5 \mathrm{~cm})$.

\section{Alterations in Quadriceps and Physical Function}

After training, lean leg mass and vastus lateralis thickness in the TKA leg increased by $4 \%$ and $14 \%$ respectively (Figure 2 ). Knee extensor strength increased by $55 \%$ (Figure 2) and the patient reported less pain with the maximal contractions.

Compared to baseline, post-training strength symmetry increased from 64 to $98 \%$ of the uninvolved leg. Changes in physical function are reported in Table 1. Most notably, the patient completed 12 more repetitions during the chair stand test, increased $40 \mathrm{~m}$ gait speed by $0.9 \mathrm{~m} / \mathrm{s}$, and improved $6 \mathrm{~min}$ walk test by $77 \mathrm{~m}$. These change scores exceeded the minimal clinically important improvement values. ${ }^{14}$ After the program, the patient reported improved gait, enhanced stair climbing/descending ability, and achieved his goal 
of resuming his walking and cycling program. Quadriceps and physical function were generally well maintained at the long-term follow-up visit (6 months post-intervention, 14 months post-TKA) (Figure 2, Table 1).

\section{B.2.5 Discussion}

\section{Feasibility and Main Findings}

Our patient was able to safely perform BFR exercise at home and tolerated the training well. To minimize the need for expensive equipment we used body weight and walking exercises that only required the use of a thigh cuff and resistance band $(\sim 30)$. We also accounted for BFR safety by using a moderate cuff pressure normalized to the patient's limb occlusion pressure. The key outcomes were that 8-weeks of BFR exercise: 1) stimulated increases in lean leg mass and vastus lateralis thickness, 2) improved knee extensor strength, and 3) enhanced physical function. Thus, implementation of homebased BFR exercise was feasible, safe, and effective.

\section{Quadriceps and Physical Function}

Restoration of quadriceps size and strength is imperative for recovery of physical function following TKA. ${ }^{4}$ In this patient, lean leg mass and vastus lateralis thickness in the TKA leg increased considerably which is noteworthy because at 6-12 months knee extensor strength is more associated with quadriceps size rather than quadriceps

activation. ${ }^{15}$ Knee extensor strength in the TKA leg also increased dramatically with BFR exercise and reached $98 \%$ of the uninvolved leg. Meier and colleagues 4 have reported 
that knee extensor strength generally increases up to 6 months post-operatively, tapers off to $\sim 80 \%$ of the uninvolved leg, and sometimes does not fully recover. Thus, the level of knee extensor strength symmetry achieved by the patient was excellent as it surpassed TKA values in the literature ${ }^{4}$ and was within the range for middle-aged ${ }^{16}$ and older adults17 ( $\geq 90 \%)$ without osteoarthritis. As expected muscle and strength gains translated to enhanced physical function as indicated by meaningful improvements in chair stand, $40 \mathrm{~m}$ fast walk, and $6 \mathrm{~min}$ walk performance. Improvements in these functional tests were consistent with the patient's self-reported increased walking, stair climbing, and cycling activity. Importantly, at the long-term follow-up visit the patient's quadriceps size and strength were still generally well maintained and physical function was still high.

\section{Possibility of BFR Exercise}

Application of BFR exercise provides a stimulus for increasing muscle size and strength in healthy ${ }^{9}$ and clinical ${ }^{8}$ populations. To date, there is a single case series ${ }^{18}$ documenting BFR exercise use after TKA (3 patients). Gaunder's team 18 reported that BFR resistance exercise implemented at $\sim 2.5$ months post-operatively increased knee extensor strength by $57-360 \%$ and restored symmetry in all 3 patients. Our case report extends upon this by highlighting the possibility of home-based BFR use and a suite of BFR-induced changes in muscle size, strength, and physical function. Collectively, these two case reports suggest that BFR exercise has potential for accelerating recovery of quadriceps and physical function after TKA. The use of BFR with this population was also proposed in a review ${ }^{10}$ on TKA rehabilitation. While BFR exercise prescription is not established for TKA it seems that it could be used in the clinic or at home and 
through resistance, aerobic, or some combination of exercise modes. A next step is to investigate these possibilities through randomized controlled studies.

\section{Limitations}

It is important to point out that the patient was middle-aged and had a rather high level of physical function as indicated by his physical activity program and performance during the baseline functional tests. Thus, results may not be the same for older and/or less active adults with TKA. The patient also had significant osteoarthritis in his contralateral right knee and thus muscle thickness and strength comparisons need be interpreted with caution. Further, while quadriceps size and strength do contribute to improved physical function there are additional factors that warrant consideration (e.g., pain, quadriceps activation, hip strength). We also acknowledge that BFR exercise induces slight discomfort and dull pain at the site of the cuff which could affect patient motivation and compliance. Partial restriction of blood flow during exercise does have potential risks inducing muscle soreness, adverse cardiovascular responses, blood clotting, and muscle/nerve damage. For this reason, its application presents a challenge. ${ }^{19}$ A systematic review and meta-analysis8 highlighted the effectiveness of using BFR exercise in a range of clinical populations and adverse effects were rare. ${ }^{20}$ With careful consideration of past medical history, appropriate cuff pressure selection, close monitoring of training, and patient education, positive BFR exercise outcomes can be achieved safely.

\section{Conclusion}


An 8-week home-based program consisting of body weight and walking exercises with BFR increased lean leg mass, vastus lateralis thickness, knee extensor strength, and functional mobility in a patient who was 6 months post-TKA. To our knowledge, this is the first and only report to describe the use of BFR at home for a TKA patient that was discharged from physical therapy and demonstrate a combination of structural and functional improvements. These results suggest that research is needed to determine the efficacy of using BFR exercise after TKA.

\section{ACKNOWLEDGMENTS}

Our research involving application of BFR exercise with clinical populations was supported by a Student Award Program Grant from the Blue Cross Blue Shield of Michigan Foundation. 


\section{REFERENCES}

1. Kurtz S., Ong K., Lau E., et al. Projections of primary and revision hip and knee arthroplasty in the United States from 2005 to 2030. J Bone Joint Surg Am. 2007;89:780785.

2. Losina E., Walensky R.P., Kessler C.L., et al. Cost-effectiveness of total knee arthroplasty in the United States: patient risk and hospital volume. Arch Intern Med. 2009;169:1113-1121; discussion 1121-1112.

3. Dusad A., Pedro S., Mikuls T.R., et al. Impact of total knee arthroplasty as assessed using patient-reported pain and health-related quality of life indices: rheumatoid arthritis vs. osteoarthritis. Arthritis Rheumatol. 2015;67:2503-2511. 
4. Meier W., Mizner R.L., Marcus R.L., et al. Total knee arthroplasty: muscle impairments, functional limitations, and recommended rehabilitation approaches. J Orthop Sports Phys Ther. 2008;38:246-256.

5. Yoshida Y., Mizner R.L., Ramsey D.K., et al. Examining outcomes from total knee arthroplasty and the relationship between quadriceps strength and knee function over time. Clin Biomech (Bristol, Avon). 2008;23:320-328.

6. Petterson S.C., Mizner R.L., Stevens J.E., et al. Improved function from progressive strengthening interventions after total knee arthroplasty: a randomized clinical trial with an imbedded prospective cohort. Arthritis Rheum. 2009;61:174-183.

7. Horlings C.G., van Engelen B.G., Allum J.H., et al. A weak balance: the contribution of muscle weakness to postural instability and falls. Nat Clin Pract Neurol. 2008;4:504-515.

8. Hughes L., Paton B., Rosenblatt B., et al. Blood flow restriction training in clinical musculoskeletal rehabilitation: a systematic review and meta-analysis. Br J Sports Med. 2017;51:1003-1011.

9. Scott B.R., Loenneke J.P., Slattery K.M., et al. Exercise with blood flow restriction: an updated evidence-based approach for enhanced muscular development. Sports Med. 2015;45:313-325.

10. Franz A., Queitsch F.P., Behringer M., et al. Blood flow restriction training as a prehabilitation concept in total knee arthroplasty: A narrative review about current 
preoperative interventions and the potential impact of BFR. Med Hypotheses. 2018;110:53-59.

11. Martin-Hernandez J., Marin P.J., Menendez H., et al. Changes in muscle architecture induced by low load blood flow restricted training. Acta Physiol Hung. 2013;100:411-418.

12. Palmer M.L., Epler M.E., Epler M.F. Fundamentals of Musculoskeletal Assessment Techniques 2nd ed. Washington Square, PA: Lippincott-Raven; 1998.

13. Dobson F., Hinman R.S., Roos E.M., et al. OARSI recommended performancebased tests to assess physical function in people diagnosed with hip or knee osteoarthritis. Osteoarthritis Cartilage. 2013;21:1042-1052.

14. Dobson F., Bennell K., Hinman R.S., et al. Recommended performance-based tests to assess physical function in people diagnosed with hip or knee osteoarthritis. Osteoarthritis Research Society International. The Univeristy of Melbourne. https://www.oarsi.org/sites/default/files/docs/2013/manual.pdf.

15. Meier W.A., Marcus R.L., Dibble L.E., et al. The long-term contribution of muscle activation and muscle size to quadriceps weakness following total knee arthroplasty. J Geriatr Phys Ther. 2009;32:79-82. 
16. Carabello R.J., Reid K.F., Clark D.J., et al. Lower extremity strength and power asymmetry assessment in healthy and mobility-limited populations: reliability and association with physical functioning. Aging Clin Exp Res. 2010;22:324-329.

17. Skelton D.A., Kennedy J., Rutherford O.M. Explosive power and asymmetry in leg muscle function in frequent fallers and non-fallers aged over 65. Age Ageing. 2002;31:119-125.

18. Gaunder C.L., Hawkinson M.P., Tennent D.J., et al. Occlusion training: pilot study for postoperative lower extremity rehabilitation following primary total knee arthroplasty. US Army Med Dep J. 2017:39-43.

19. Spranger M.D., Krishnan A.C., Levy P.D., et al. Blood flow restriction training and the exercise pressor reflex: a call for concern. Am J Physiol Heart Circ Physiol. 2015;309:H1440-1452.

20. Nakajima T., Kurano M., Iida H. Use and safety of KAATSU training: results of a national survey. Int J KAATSU Train Res. 2006;2:5-13. 
Table 1: Alterations in Physical Function

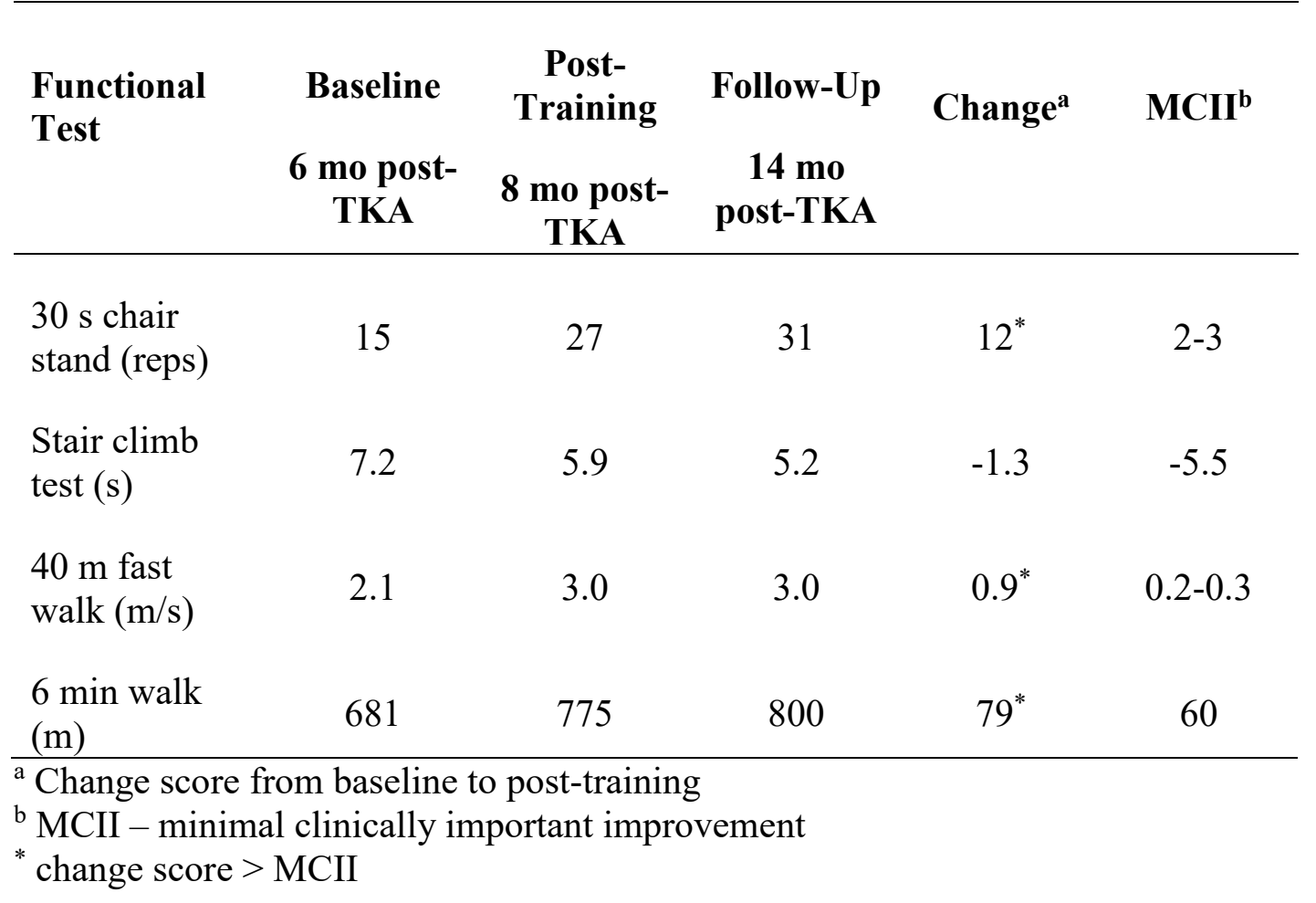




\section{FIGURE CAPTIONS}

Figure 1: Overview of the home-based BFR program consisting of body weight and walking exercises. The patient performed this $\sim 25 \mathrm{~min}$ exercise routine $5 \mathrm{x} / \mathrm{wk}$ for $8 \mathrm{wks}$.

Figure 2: Alterations in lean leg mass (A), vastus lateralis (VL) muscle thickness (B), and knee extensor strength (C). 


\section{Single-Leg Knee Extension}

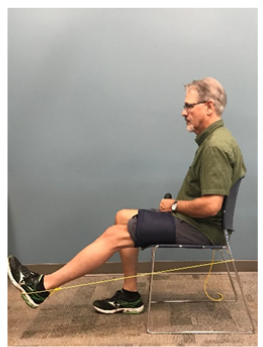

- Inflate cuff to $50 \%$ of LOP

- 3 sets of 30 reps $\left(90-0^{\circ}\right)$

- 1 min rest between sets

- Deflate cuff after final set

Exercise TKA leg only, adjust resistance as needed

\section{Body Weight Half Squats}

- Inflate cuff to $50 \%$ of LOP

- 3 sets of 30 reps $\left(0-45^{\circ}\right)$

- 1 min rest between sets

- Deflate cuff after final set

Cuff placed around TKA leg only

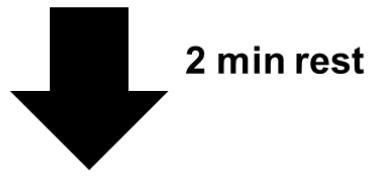

\section{Walking}

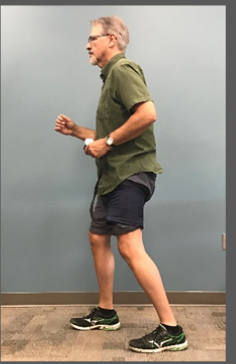

- Inflate cuff to $50 \%$ of LOP

- 3 sets of 2 min intervals

- 1 min rest between sets

- Deflate cuff after final set

\section{Cuff placed around TKA leg only}



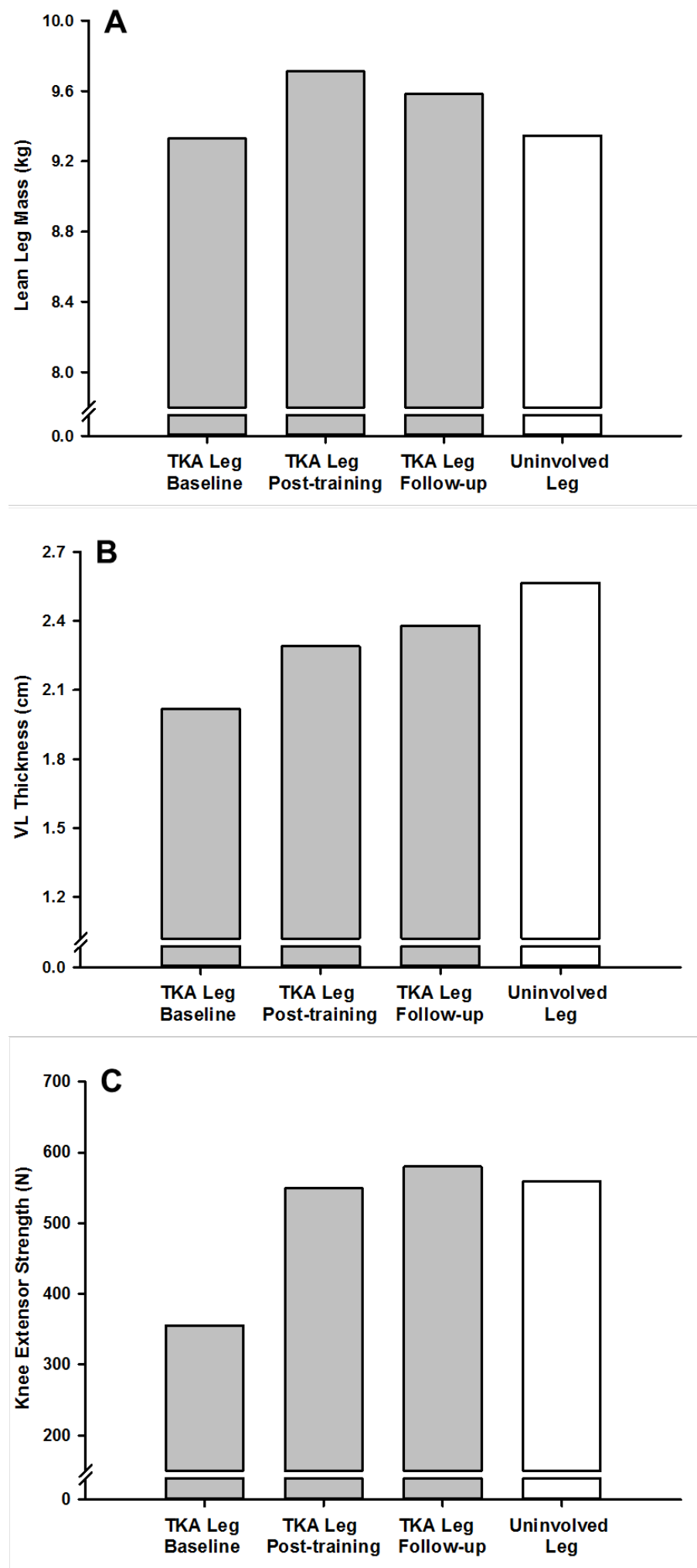


\section{Grants Submitted}

\section{C.1 Blue Cross Blue Shield of Michigan Foundation}

\section{PROPOSAL}

Anterior cruciate ligament (ACL) injury is the most common traumatic knee injury with approximately 200,000 new injuries each year. ${ }^{1,2}$ However, only about $50 \%$ of patients are able to return to their pre-injury activity level. ${ }^{3}$ This may be because many patients do not completely regain their quadriceps function (i.e., muscle size and strength), resulting in limb asymmetry. Over time limb asymmetry negatively alters the loading patterns on the knee which ultimately increases risk for re-injury and early onset of osteoarthritis. ${ }^{4,5}$ Osteoarthritis of the knee is more common in individuals who previously had ACL tears. ${ }^{6,7}$ It was estimated that annual costs attributed to osteoarthritis, in those with ACL reconstruction, is $\$ 2.78$ billion. $^{8}$

High load resistance exercise ( $70 \%$ of one repetition maximum, "heavy weight") is effective for improving quadriceps muscle size and strength. ${ }^{9,10}$ However, many ACL patients are advised not to perform high load resistance exercise because doing so could compromise the repaired joint by increasing stress and strain on the ACL. Recent evidence suggests that moderate restriction of blood flow during low load resistance exercise is a potent stimulus for increasing quadriceps muscle size and strength in healthy and clinical populations. ${ }^{9,13}$ Blood flow restricted (BFR) exercise involves using low load resistance exercise ( $20 \%$ of one repetition maximum, "light weight") while using an occlusion cuff to reduce venous return. ${ }^{9}$ Muscles are experiencing the benefits of high load resistance training without putting excessive stress on the repaired joint. Although 
BFR has been used in ACL rehabilitation in the past, ${ }^{10}$ it is unclear if BFR could be implemented several years after ACL injury in a home-based exercise program to reverse chronic deficits in quadriceps size and strength and restore limb symmetry. The purpose of this project is to test the hypothesis that four weeks of BFR exercise will increase quadriceps size and strength and reduce quadriceps asymmetry. This research is significant because it will extend upon previous ACL studies, overcome major rehabilitation barriers, fill gaps with long term recovery, and support BCBSM Foundation initiatives to improve the health of Michigan residents.

I will use a two-group, pre/post, longitudinal study design with 20 participants. Eligible and consented individuals will complete: 1) baseline measurements, 2) a four week BRF exercise intervention, and 3) post-training measurements. Baseline measures of quadriceps muscle size, quadriceps strength, and functional task performance will be assessed. Participants will be randomly assigned to either a blood flow restricted exercise $(\mathrm{BFR} ; \mathrm{n}=10)$ or control $(\mathrm{CON} ; \mathrm{n}=10)$ group. BFR group will train for $5 \mathrm{x} /$ week $(30$ $\mathrm{min} / \mathrm{session}$ ) for 4 weeks with the blood pressure cuff inflated to $80 \%$ of the occlusion pressure. The program will consist of home-based exercises that have been shown to increase quadriceps size and strength. ${ }^{10,15} \mathrm{CON}$ group will not perform exercise training. Baseline measures will be assessed one week after completion of the program. Outcome measures of quadriceps size and strength and one legged functional hop height, will be used to test the effectiveness of the BFR program. Secondary outcome measures of completed sessions, pain, and satisfaction will be used to test the practicality of BFR in a home setting. 
My research will establish BFR as an effective exercise mode to restore limb symmetry after an ACL injury and reduce associated risks, such as osteoarthritis and re-injury. This home-based exercise program will help to facilitate better access to care and reduce costs associated with prolonged physical therapy. This research will have applications in rehabilitation when high load resistance training is contraindicated. The research results will be disseminated through my doctoral dissertation, a presentation at the national meeting for the American College of Sports Medicine, and a publication in the Journal of Orthopedic \& Sports Physical Therapy.

\section{REFERENCES}

1. Baquie P, Brukner P. Injuries presenting to an Australian sports medicine centre: a 12-month study. Clin J Sport Med. 1997;7(1):28-31.

2. Paterno MV, Rauh MJ, Schmitt LC, Ford KR, Hewett TE. Incidence of Second ACL Injuries 2 Years After Primary ACL Reconstruction and Return to Sport. Am J Sports Med. 2014;42(7):1567-1573.

3. Kvist J. Rehabilitation following anterior cruciate ligament injury: current recommendations for sports participation. Sports Med. 2004;34(4):269-280.

4. Chmielewski TL. Asymmetrical lower extremity loading after ACL reconstruction: more than meets the eye. J Orthop Sports Phys Ther. 2011;41(6):374-376. 
5. Salmon L, Russell V, Musgrove T, Pinczewski L, Refshauge K. Incidence and risk factors for graft rupture and contralateral rupture after anterior cruciate ligament reconstruction. Arthroscopy. 2005;21(8):948-957.

6. Oiestad BE, Holm I, Aune AK, et al. Knee function and prevalence of knee osteoarthritis after anterior cruciate ligament reconstruction: a prospective study with 10 to 15 years of follow-up. Am J Sports Med. 2010;38(11):2201-2210.

7. Oiestad BE, Holm I, Engebretsen L, Risberg MA. The association between radiographic knee osteoarthritis and knee symptoms, function and quality of life 10-15 years after anterior cruciate ligament reconstruction. Br J Sports Med. 2011;45(7):583588.

8. Mather RC, 3rd, Koenig L, Kocher MS, et al. Societal and economic impact of anterior cruciate ligament tears. J Bone Joint Surg Am. 2013;95(19):1751-1759.

9. Scott BR, Loenneke JP, Slattery KM, Dascombe BJ. Exercise with blood flow restriction: an updated evidence-based approach for enhanced muscular development. Sports Med. 2015;45(3):313-325.

10. Ohta H, Kurosawa H, Ikeda H, Iwase Y, Satou N, Nakamura S. Low-load resistance muscular training with moderate restriction of blood flow after anterior cruciate ligament reconstruction. Acta Orthop Scand. 2003;74(1):62-68.

11. Palmitier RA, An KN, Scott SG, Chao EY. Kinetic chain exercise in knee rehabilitation. Sports Med. 1991;11(6):402-413. 
12. Fitzgerald GK. Open versus closed kinetic chain exercise: issues in rehabilitation after anterior cruciate ligament reconstructive surgery. Phys Ther. 1997;77(12):17471754.

13. Manini TM, Clark BC. Blood flow restricted exercise and skeletal muscle health. Exerc Sport Sci Rev. 2009;37(2):78-85.

14. Lejkowski PM, Pajaczkowski JA. Utilization of Vascular Restriction Training in post-surgical knee rehabilitation: a case report and introduction to an under-reported training technique. J Can Chiropr Assoc. 2011;55(4):280-287.

15. Abe T, Kearns CF, Sato Y. Muscle size and strength are increased following walk training with restricted venous blood flow from the leg muscle, Kaatsu-walk training. J Appl Physiol (1985). 2006;100(5):1460-1466.

\section{C.2 Michigan Space Grant Consortium}

Background: President Obama has set a clear goal to send humans to Mars by 2030. With current propulsion systems, it would take six months to reach Mars. However, with this long duration trip to Mars, astronauts' musculoskeletal and cardiovascular systems would be severely compromised; it is estimated that astronauts would lose almost half of their muscle mass. ${ }^{1}$ These impairments of physiological function would not only affect performance of mission tasks, but make a return to Earth's environment impossible as astronauts could no longer withstand Earth's gravity. ${ }^{1}$ Therefore, before long term space travel is deemed safe for astronauts, effective exercise countermeasures must be 
developed, tested, and employed. To counteract the losses in physiological function astronauts are scheduled for 2.5 hours of daily physical training. Specifically, astronauts perform 1.5 hours of resistance training (e.g., lifting weights), and 1 hour of aerobic training (e.g., cardiovascular exercise).2 Although the exercise devices on the International Space Station can attenuate these impairments, physiological degradation still occurs.2 As NASA prepares for long-duration space exploration, it is imperative that new exercises preformed during spaceflight be light-weight, robust, and target musculoskeletal and cardiovascular systems.

Project Description: A novel method to maintain the levels of musculoskeletal and cardiovascular conditioning during long term spaceflight could be blood flow restricted exercise training (BFR). ${ }^{2}$ As illustrated in the figure to the right, BFR involves lifting light weights ( $20 \%$ of one repetition maximum) while simultaneously using a blood pressure cuff to reduce blood flow out of the leg. In other words, blood flow is traveling to the working muscles but not all of it is returning to the heart. ${ }^{3}$ Evidence indicates that BFR training is effective in increasing muscle size and strength and diminishing disuse muscle loss. BFR causes a build-up of metabolites within the muscle and stimulates the production of growth hormone and other growth factors that increase muscle protein synthesis. ${ }^{2}$ Blood flow restriction during walking and cycling has not only been shown to increase muscle size and strength, but also improve the cardiovascular system (i.e., increase exercise capacity) ${ }^{4-10}$ Increased exercise capacity may be due peripheral factors such as increases in capillary density, and muscle enzyme oxidative capacity ${ }^{5}$ or central factors such as an increased submaximal stroke volume. ${ }^{6}$ These results indicate that it 
may be possible for BFR to be a 2 for 1 activity; in which astronauts using BFR during aerobic training maintain musculoskeletal function (muscle size and strength) and cardiovascular endurance (exercise capacity).

While the potential advantages of using BFR have been highlighted, NASA has stated that, "exercise prescription for BFR remains incomplete, and more scientific data at the whole body and cell level are needed to further elucidate the best exercise paradigm."11 To create effective exercise prescription for BFR it is critical to understand how muscle contractile function (i.e., level of muscular fatigue, location of that fatigue, and time course of recovery) changes after an acute exercise session with BFR. Identifying acute changes in muscle contractile function will aid in determining 1) duration and frequency of aerobic exercise with BFR and 2) how much recovery is needed following a workout. Acute changes in muscle function have been assessed following resistance exercise with BFR, but not following aerobic exercise with BFR.

Aim: To determine acute changes in muscle contractile function following aerobic exercise with BFR and compare to those following aerobic exercise without BFR. I hypothesize that aerobic exercise with BFR will require more muscle activity and result in larger reductions in post exercise maximal voluntary torque compared to aerobic exercise without BFR, which acts as a potent stimulus for increased musculoskeletal and cardiovascular conditioning. This project will improve our understanding of acute responses to aerobic exercise with BFR and aid with developing robust exercise countermeasures which align with NASA's long term goals. 
Methods: 16 recreationally active males and females will participate in this study. Muscle contractile function will be assessed before and after cycling exercise, to understand acute central and peripheral adaptations to cycling with BFR. Eligible and consented participants will undergo a familiarization session, followed by two exercise sessions in which the participants will cycle at $30 \%$ of their peak work rate for $15 \mathrm{~min}$ with and without BFR. Measures of peak isometric torque, muscle activation, and central and peripheral mechanisms of fatigue will be recorded before and after exercise. These measures will be recorded $30 \mathrm{secs}, 2 \mathrm{~min}, 3 \mathrm{~min}, 4 \mathrm{~min}$, and $5 \mathrm{~min}$ post-exercise. Central fatigue will be quantified as the pre- to post-exercise reduction in voluntary activation whereas peripheral fatigue will be quantified as pre- to post-exercise reduction in evoked torque. A within subjects repeated measures ANOVA will determine statistical significance $(\mathrm{P}<0.05)$.

NASA's Strategic Interest: Objective 1.1 of NASA's Strategic Plan is to expand the human presence into the solar system and to Mars. This exploration requires the ability to transport crew further, and for longer duration than previously attempted. With current technology it would take six months for a crew to reach Mars and then astronauts would have to leave within 30 days, or stay for over 500 days. Long duration habitation in microgravity environments would cause many physiological adaptions, including muscle loss and adverse cardiovascular adaptation. ${ }^{1}$ Development of aerobic exercise with BFR for spaceflight would be inexpensive, easy to implement, and compatible with current NASA exercise equipment. ${ }^{2,4,12}$ At this time musculoskeletal and cardiovascular adaptations to exercise vary depending upon the exercise mode (aerobic training vs. 
resistance training) and the timing relative to successive exercise trials. These concepts need to be understood better to develop a BFR exercise countermeasure. ${ }^{11}$

Project Role and Outcomes: As the project leader, I will recruit subjects, conduct all exercise trials and experimental measures, and analyze data. Experimental results will be disseminated through my doctoral dissertation, a presentation at the regional meeting for the Michigan Physiological Society and national meeting for the American College of Sports Medicine, and a publication in the Journal of Applied Physiology. I will hold weekly meetings with Dr. Elmer and Dr. Yoon (Co-PI's) to discuss progress and ensure that these outcomes are reached. I will also consult with Dr. Cook, a BFR expert, throughout the duration of the project.

Future Career Interests: I have a Bachelor of Science in Biomedical Engineering, a Master of Science in Exercise Science, and I am currently pursuing a PhD in Biology. With my unique background I will become a university professor specializing in rehabilitation science and find better ways to restore musculoskeletal function, maintain health, and improve performance in healthy and clinical populations. Currently, I am leading a National Science Foundation funded project to develop new exercise equipment and a countermeasure to improve the mobility of wheelchair users. Further, for my dissertation I am using BFR as a novel exercise program to overcome barriers associated with traditional exercise training and improve health and performance. This study aligns with the vision of the MSGC to foster awareness and support space-related research in Michigan. Finally, support from MSGC will serve as a crucial step to establish aerobic training with BFR and improve exercise prescription for astronauts. 


\section{References}

1. Hawkey A. The importance of exercising in space. Interdiscip Sci Rev. $2003 ; 28(2): 130-138$

2. Hackney KJ, Everett M, Scott JM, Ploutz-Snyder L. Blood flow-restricted exercise in space. Extrem Physiol Med. 2012;1(1):12.

3. Scott BR, Loenneke JP, Slattery KM, Dascombe BJ. Exercise with blood flow restriction: an updated evidence-based approach for enhanced muscular development. Sports Med. 2015;45(3):313-325.

4. de Oliveira MF, Caputo F, Corvino RB, Denadai BS. Short-term low-intensity blood flow restricted interval training improves both aerobic fitness and muscle strength. Scand J Med Sci Sports. 2016;26(9):1017-1025.

5. Abe T, Kearns CF, Sato Y. Muscle size and strength are increased following walk training with restricted venous blood flow from the leg muscle, Kaatsu-walk training. J Appl Physiol (1985). 2006;100(5):1460-1466.

6. Park S, Kim JK, Choi HM, Kim HG, Beekley MD, Nho H. Increase in maximal oxygen uptake following 2-week walk training with blood flow occlusion in athletes. Eur J Appl Physiol. 2010;109(4):591-600.

7. Abe T, Kearns CF, Fujita S, Sakamaki M, Sato Y, Brechue WF. Skeletal muscle size and strength are increased following walk training with restricted leg muscle blood 
flow: implications for training duration and frequency. International Journal of KAATSU Training Research. 2009;5(1):9-15.

8. Abe T, Fujita S, Nakajima T, et al. Effects of Low-Intensity Cycle Training with Restricted Leg Blood Flow on Thigh Muscle Volume and VO2MAX in Young Men. J Sports Sci Med. 2010;9(3):452-458.

9. Abe T, Sakamaki M, Fujita S, et al. Effects of low-intensity walk training with restricted leg blood flow on muscle strength and aerobic capacity in older adults. $\mathrm{J}$ Geriatr Phys Ther. 2010;33(1):34-40.

10. Corvino RB, Oliveira MFMd, Santos RPd, Denadai BS, Caputo F. Four weeks of blood flow restricted training increases time to exhaustion at severe intensity cycling exercise. Rev. bras. cineantropom. desempenho hum. 2014;16(5):570-578.

11. In: Scott-Conner CEH, Masys DR, Liverman CT, eds. Review of NASA's Evidence Reports on Human Health Risks: 2015 Letter Report. Washington (DC)2016.

12. Takarada Y, Takazawa H, Ishii N. Applications of vascular occlusion diminish disuse atrophy of knee extensor muscles. Med Sci Sports Exerc. 2000;32(12):2035-2039.

\section{C.3 American College of Sports Medicine Student Award Program}

Significance: Public Health - Currently $80 \%$ of US adults do not meet the necessary guidelines for both aerobic and resistance training (3). Resistance training has a profound effect on the development and maintenance of muscular strength, this improves 
functional abilities, prevents osteoporosis, sarcopenia, and low-back injuries. In addition resistance training may help to prevent metabolic diseases such as diabetes, cardiovascular disease, and cancer by improving insulin sensitively, resting metabolic rate, blood pressure, and body fat (4). Accordingly, resistance training is universally recommended by the American College of Sports Medicine, the Center for Disease Control, and the National Strength and Conditioning Association, for development of strength, work performance, disease prevention, and healthy aging. As a result guidelines and exercise prescription for resistance training have been developed. Failure to meet these guideline has a large economic impact, it has been estimated that the direct cost of sarcopenia alone in the U.S. is approximately $\$ 18.5$ billion dollars annually (1). In general $65-70 \%$ one repetition maximum $(\mathrm{RM})$ exercise performed 2-3 times per week for a minimum of 6-8 weeks is necessary to increase muscle size and strength $(10,13,14)$. This type of training is not always possible in certain clinical populations, where the high training loads are contraindicated or impossible (8). Thus, alternative methods for developing muscular strength are needed.

$\neg$ Blood flow restriction exercise - Resistance exercise with blood flow restriction is emerging as an effective alternative option to build strength in healthy, clinical, and athletic populations. This exercise modality involves the use of mechanical compression of the limb, usually with an inflatable cuff or tourniquet, used in combination with much lower loads than traditional resistance training (e.g., 20\% vs. 70\% 1 RM ) (14). The two main advantages that blood flow restriction exercise has over traditional resistance 
training are: 1) increases in muscle size and strength are elicited at low training loads and 2) these adaptations occur faster with blood flow restriction. The development of muscle size and strength under low loads is significant because in many clinical cases high loads cannot be used. One population includes people post-surgery, where high loads may compromise the repaired tissue (13). In addition, those with neurological disorders such as Cerebral Palsy, where the patient is not able to fully activate the target muscle (8). Wounded soldiers and injured athletes may also see great benefits by returning to activity much quicker than by traditional resistance training (11). Finally older adults can also benefit by getting a similar training stimulus of more difficult exercises, without the need for higher levels of fitness (17).

Barriers and Gaps - While blood flow restriction training has many advantages there are several barriers preventing widespread implementation. First, there are no recommendations for the optimal occlusion cuff size and pressure. Second, there is a lack of clinical trials documenting the relative safety of blood flow restriction training $(8,14)$. Although injuries during blood flow restriction training are rare (12), it does place many stressors on the cardiovascular system (15). Improper application of this training could cause detrimental side effects including adverse cardiovascular responses, changes in blood clotting or vascular function, and muscle and/or nerve damage (8). To minimize these risks it is essential to individualize the occlusion cuff pressure however, there are no standardized recommendations to do this $(6,14)$. Thus, the lack of specific exercise prescription guidelines is a critical barrier to the widespread use of blood flow restriction 
training. Ideally the pressure in the occlusion cuff should be high enough to prevent venous return, but low enough as to maintain arterial flow into the muscle (7) (see Figure 1). Currently, the optimal occlusion cuff pressure is unknown. If the pressure is set to low, the training stimulus may not be strong enough to enhance muscle function. If the pressure is too high, safety issues may arise. To my knowledge there are only two reports of using Doppler ultrasound to measure blood flow before and immediately after resistance exercise with blood flow restriction (14). These authors reported no difference in blood flow measured before and after exercise with blood flow restriction (16), and that increased occlusion cuff pressure decreased blood flow (5). However, these researchers did not measure blood flow during exercise. It is well known that blood pressure, cardiac output and blood flow to the working muscle all increase significantly with exercise (9). Thus, a significant gap exists in our understanding of how limb blood flow changes during dynamic exercise with blood flow restriction. Before comprehensive blood flow restriction training guidelines are made it is essential that the mechanism of blood flow during dynamic exercise with blood flow restriction is determined. Accordingly, the expected contribution of this research is to determine the effects of occlusion cuff pressure and size on limb blood flow during dynamic exercise (involving locomotor muscles) with blood flow restriction.

Overall Significance - The proposed research is significant because it focuses on an important public health problem and aims to overcome barriers to the widespread use of blood flow restriction training (the lack of specific guidelines clinical trials). The proposed research is clinically significant given the potential widespread use of blood 
flow restriction training and the potential serious issues that may arise if blood flow restriction training is improperly conducted. Furthermore, the proposed research will fill a significant gap in our understanding of the mechanisms of blood flow during dynamic exercise with blood flow restriction training. The broader impact of this research is to advance exercise prescription of blood flow restriction training by identifying changes in blood flow during exercise with blood flow restriction. This will better identify the optimal occlusion cuff pressure and size. If the specific aims are achieved, the proposed research could enable a wider range of individuals to use blood flow restriction training, by increasing the relative safety of this exercise modality. The scientific knowledge gathered from this research will have practical applications for clinicians, researchers, and applied sport scientists within the field of exercise science and sports medicine, which is in line with the Mission of the American College of Sports Medicine.

Pilot data - As a first step I measured blood flow during rhythmic handgrip exercise with blood flow restriction using the technique proposed in this investigation on a small sample of participants $(\mathrm{N}=5)$. I measured blood flow in the brachial artery $5 \mathrm{~cm}$ below the occlusion cuff set at $50 \%$ of the occlusion pressure measured prior to experiment. Results indicated that forearm blood flow increased by $46 \pm 9 \%$ from rest during exercise with blood flow restriction. These results suggest there is a difference in blood flow during exercise with blood flow restriction. Moreover, this is the first to confirm blood flow is maintained during blood flow restriction exercise, but the flow is less than exercise without blood flow restriction. This supports the theoretical mechanism of blood flow restricted exercise. Moving forward, it is essential that this approach be replicated during 
dynamic exercise involving locomotor muscles (knee extensors) under different occlusion cuff pressures and widths.

Innovation: I believe that the proposed research is very innovative because it focuses on improving our understanding of blood flow restriction training to advance the exercise prescription of this training. Specifically, I will be measuring femoral blood flow during dynamic knee extension exercise with blood flow restriction, which no other blood flow restriction study has done. The proposed study will overcome traditional barriers to this research by non-invasively measuring blood flow using a Doppler Ultrasound. Moreover, while previous research employs a standard pressure among all participants, we believe this is insufficient and potentially dangerous. The information gleaned from this study addresses fundamental gaps in blood flow restriction training and is necessary to establish a comprehensive blood flow restriction prescription including optimal occlusion cuff sizes and pressures. To date, I have piloted this approach during dynamic exercise involving a small muscle mass, and will extend this to dynamic exercise involving locomotor muscles, which will be clinically relevant. The proposed research is necessary to develop more robust exercise prescription guidelines for blood flow restriction training.

Approach: Research Team - Together my research group is well positioned to perform the proposed study. I have a background in both biomedical engineering (BS) and exercise physiology (MS). This has prepared me to move forward with my doctoral dissertation on using blood flow restriction exercise to restore musculoskeletal function, maintain health, and improve performance in healthy and clinical populations. My 
dissertation advisor, Dr. Steven Elmer, Assistant Professor in the Department of Kinesiology and Integrated Physiology at Michigan Tech University, and I have been working together for two years and currently are funded to complete a project using blood flow restriction exercise in individuals with chronic quadriceps asymmetry due to ACL reconstruction. Dr. John McDaniel, Assistant Professor in the School of Health Sciences at Kent State University, has worked with Dr. Elmer on many collaborative projects (see letter of support), and has provided me with hands-on training for the measurement of femoral blood flow during dynamic exercise. Dr. McDaniel has produced several publication using this measurement. With this team, I have the mentorship needed to carry out this project.

Participants - For this project I will recruit 15 young healthy active individuals (sample size estimation described below) from the surrounding community to participate in this study. Participants will be excluded if they: have any physical limitations, are a smoker, have diabetes, have any cardiopulmonary disorders including but not limited to hypertension. Participants will provide written, informed consent approved by the Michigan Technological University Institutional Review Board prior to study enrollment. All participants will attend an orientation session before the start of the study for an overview of the experimental protocol and familiarization with the equipment and procedures.

Methods - During the familiarization visit, eligible and consented participants will become familiar with the measurement of blood flow using Doppler Ultrasound. Femoral blood flow will be measured based on the techniques described by Burns and colleagues 
(2) using B-mode ultrasound (GE Logiq e BT12, GE Health Care, Chicago, IL, USA). A $12 \mathrm{MHz}$ scanning head will be placed over the femoral artery midway between the anterior superior iliac spine and the symphysis pubis. The occlusion cuff will be placed just below the scanning head. Artery radius (r) and mean blood velocity (MBV) with be recorded continuously. Blood flow (BF) will be calculated as BF $=\mathrm{MBV} \mathrm{X} \pi \mathrm{r} 2$. Occlusion pressure at rest will be analyzed with the participant laying supine on an examination table. The occlusion cuff will be placed in the same position as above. The investigator will inflate the occlusion cuff starting at $100 \mathrm{mmHG}$ and increasing the pressure by $10 \mathrm{mmHg}$ until no blood flow can be detected in the anterior tibial artery using the Doppler Ultrasound. The investigator will then slowly deflate the occlusion cuff until blood flow resumes. One repetition maximum will also be assessed on a knee extension ergometer developed for this study, which will also be used during the exercise session. Participants will be seated with their back angled at $10^{\circ}$. The participant will be asked to extend their knee against a load. If the participant can successfully lift the load 10 times, they will be given a 5 minute rest and the load will increase. This process will continue until the subject can no longer lift the load ten times. One repetition maximum will then be estimated using the Brzycki Equation $1 \mathrm{RM}=$ weight X $0.9729 \mathrm{X}$ Repetitions. Following the familiarization visit participants will complete two exercise sessions. Participants will be asked to perform five sets of 30 knee extensions at $20 \%$ of their one repetition. The pressure in the occlusion cuff will be randomly set to $0 \%, 50 \%$, $75 \%, 100 \%$, and $125 \%$ of the occlusion pressure at rest. Blood flow at rest will be measured for 1 minute prior to exercise. Participants will then be asked to perform these exercises at a set cadence, while femoral blood flow is assessed. Participants will be 
given 10 minutes rest between sets. Participants will be assigned to which occlusion cuff width $(5 \mathrm{~cm}, 18 \mathrm{~cm})$ they perform in a counterbalanced order

Statistical Analyses - Given a power of 0.8 , an effect size of 0.8 , and an alpha of 0.05 , a sample of 15 participants will provide an adequate sample. To test aim 1: A two- way analysis of variance (ANOVAs) will be used to test the effect of pressure and exercise on femoral blood flow. To test aim 2: a one way ANOVA with repeated measures will be used to assess the effect of cuff width. The level of significance will be set at $p<0.05$.

Potential Problems -The use of Doppler Ultrasound to measure blood flow is a new measurement for my laboratory. Collectively, Dr. Elmer and I have received training from the manufacture, attended an ultrasound workshop, and received hands on training from Dr. John McDaniel (trained under cardiovascular exercise physiologist Dr. Russell Richardson, Professor in the Department of Internal Medicine, University of Utah and Salt Lake VA Medical Center) who has a long publication history with this type of measurement (see letter of support). In short, Dr. Elmer's laboratory has gone to great lengths to ensure that we have the background, mentorship, and resources needed to overcome this challenge. There is also potential for injury when performing blood flow restriction exercise. In a national Japanese survey of facilities using blood flow restricted exercise in which over 13,000 individuals engaged in training various modes of blood flow restricted exercise (walking, cycling, weight training), the most common side effects were bruising at the site of the tourniquet, or occlusion cuff (incidence $=13.1 \%$ ) and 
temporary numbness $(1.3 \%)$. More serious side effects were rare and included: venous thrombosis $(0.055 \%)$, cerebral infarction $(0.008 \%)$, rhabdomyolysis $(0.008 \%)$ and pulmonary embolism (0.008\%) (12). Although these events are extremely rare, it should be noted that determining the relative safety of blood flow restriction requires a detailed study of several potential outcomes, and we acknowledge that unforeseen risks do exist. Dr. Elmer's laboratory has talked with Dr. Summer Cook, Associate Professor in the Department of Kinesiology at the University of New Hampshire, regarding the protocols and safety precautions for utilizing blood flow restricted exercise. Dr. Cook has received funding from the National Institutes of Health to use blood flow restricted exercise with frail elderly individuals and also serves on her University's IRB review board. Based on these discussions with Dr. Cook, Dr. Elmer's laboratory is well prepared to implement blood flow restriction safely.

Timeline: The research results will be disseminated through my doctoral dissertation, a presentation at the national meeting for the American College of Sports Medicine, and at least one publication (target journal Medicine \& Science in Sports \& Exercise). I aim to have $75 \%$ of the data collected at 6 months and manuscript prepared at 12 months. To ensure that the research progresses on schedule, I will have bimonthly meetings with Dr. Elmer and Dr. McDaniel. This one year project will be a key part of my dissertation, provide pilot data for a larger NIH application, and help create much needed guidelines for blood flow restriction training.

\section{References}


1. Beaudart C, Rizzoli R, Bruyère O, Reginster J-Y, Biver E. Sarcopenia: burden and challenges for public health. Archives of Public Health. 2014;72(1):1.

2. Burns KJ, Pollock BS, McDaniel J. The cardiovascular response to passive movement is joint dependent. Physiological reports. 2016;4(5):e12721.

3. Clarke TC, Ward BW, Freeman G, Schiller JS. Early release of selected estimates based on data from the January-March 2015 National Health Interview Survey. National Center for Health Statistics. 2015;September 201(5).

4. Fleck SJ, Kraemer W. Designing Resistance Training Programs, 4E. Human Kinetics; 2014.

5. Hunt JE, Stodart C, Ferguson RA. The influence of participant characteristics on the relationship between cuff pressure and level of blood flow restriction. European journal of applied physiology. 2016:1-12.

6. Jessee MB, Buckner SL, Mouser JG, Mattocks KT, Loenneke JP. Letter to the editor: Applying the blood flow restriction pressure: the elephant in the room. American Journal of Physiology-Heart and Circulatory Physiology. 2015;310(1):H132-H3.

7. Loenneke J, Thiebaud R, Abe T, Bemben M. Blood flow restriction pressure recommendations: the hormesis hypothesis. Medical hypotheses. 2014;82(5):623-6.

8. Manini TM, Clark BC. Blood Flow Restricted Exercise and Skeletal Muscle Health. Exerc Sport Sci Rev. 2009;37(2):78-85. 
9. McArdle WD, Katch FI, Katch VL. Exercise physiology: nutrition, energy, and human performance. Lippincott Williams \& Wilkins; 2010.

10. Mcdonagh MJN, Davies CTM. Adaptive Response of Mammalian SkeletalMuscle to Exercise with High Loads. Eur J Appl Physiol O. 1984;52(2):139-55.

11. McEwen J, Jeyasurya J, Owens J. HOW CAN PERSONALIZED TOURNIQUET SYSTEMS ACCELERATE REHABILITATION OF WOUNDED WARRIORS, PROFESSIONAL ATHLETES AND ORTHOPAEDIC PATIENTS? 2016.

12. Nakajima $\mathrm{T}$, Kurano M, Iida $\mathrm{H}$ et al. Use and safety of KAATSU training: results of a national survey. International Journal of KAATSU Training Research. 2006;2(1):513.

13. Ohta H, Kurosawa H, Ikeda H, Iwase Y, Satou N, Nakamura S. Low-load resistance muscular training with moderate restriction of blood flow after anterior cruciate ligament reconstruction. Acta Orthop Scand. 2003;74(1):62-8.

14. Scott BR, Loenneke JP, Slattery KM, Dascombe BJ. Exercise with blood flow restriction: an updated evidence-based approach for enhanced muscular development. Sports Med. 2015;45(3):313-25.

15. Spranger MD, Krishnan AC, Levy PD, O'Leary DS, Smith SA. Blood flow restriction training and the exercise pressor reflex: a call for concern. American Journal of Physiology-Heart and Circulatory Physiology. 2015;309(9):H1440-H52. 
16. Takano $\mathrm{H}$, Morita $\mathrm{T}$, Iida $\mathrm{H}$ et al. Hemodynamic and hormonal responses to a short-term low-intensity resistance exercise with the reduction of muscle blood flow. European journal of applied physiology. 2005;95(1):65-73.

17. Yasuda T, Fukumura K, Fukuda T et al. Muscle size and arterial stiffness after blood flow-restricted low-intensity resistance training in older adults. Scandinavian journal of medicine \& science in sports. 2014;24(5):799-806. 


\section{Copyright Documentation}

\section{SPRINGER NATURE LICENSE \\ TERMS AND CONDITIONS}

Dec 10, 2018

This Agreement between Matthew Kilgas ("You") and Springer Nature ("Springer Nature") consists of your license details and the terms and conditions provided by Springer Nature and Copyright Clearance Center.

License Number

License date

Licensed Content Publisher

Licensed Content Publication

Licensed Content Title

Licensed Content Author

Licensed Content Date

Type of Use

Requestor type

Format

Portion

Will you be translating?

Number of languages

Circulation/distribution

Author of this Springer Nature content

Title

Institution name

Expected presentation date

Specific Languages

Requestor Location
4483680159043

Dec 07, 2018

Springer Nature

European Journal of Applied Physiology

Limb blood flow and tissue perfusion during exercise with blood flow restriction

Matthew A. Kilgas, John McDaniel, Jon Stavres et al

Jan 1, 2018

Thesis/Dissertation

academic/university or research institute

print and electronic

full article/chapter

yes, including original language

1

$<501$

yes

Assistant Professor

Northern Michigan University

Dec 2018

English

Matthew Kilgas

School of Health and Human Performance

1401 Presque Isle Ave

MARQUETTE, MI 49855 
United States

Attn: Matthew Kilgas

Billing Type

Billing Address

Total

Terms and Conditions
Invoice

Matthew Kilgas

School of Health and Human Performance

1401 Presque Isle Ave

MARQUETTE, MI 49855

United States

Attn: Matthew Kilgas

0.00 USD

Springer Nature Terms and Conditions for RightsLink Permissions

Springer Nature Customer Service Centre GmbH (the Licensor) hereby grants you a non-exclusive, world-wide licence to reproduce the material and for the purpose and requirements specified in the attached copy of your order form, and for no other use, subject to the conditions below:

1. The Licensor warrants that it has, to the best of its knowledge, the rights to license reuse of this material. However, you should ensure that the material you are requesting is original to the Licensor and does not carry the copyright of another entity (as credited in the published version).

If the credit line on any part of the material you have requested indicates that it was reprinted or adapted with permission from another source, then you should also seek permission from that source to reuse the material.

2. Where print only permission has been granted for a fee, separate permission must be obtained for any additional electronic re-use.

3. Permission granted free of charge for material in print is also usually granted for any electronic version of that work, provided that the material is incidental to your work as a whole and that the electronic version is essentially equivalent to, or substitutes for, the print version.

4. A licence for 'post on a website' is valid for 12 months from the licence date. This licence does not cover use of full text articles on websites.

5. Where 'reuse in a dissertation/thesis' has been selected the following terms apply: Print rights of the final author's accepted manuscript (for clarity, NOT the published version) for up to 100 copies, electronic rights for use only on a personal website or institutional repository as defined by the Sherpa guideline (www.sherpa.ac.uk/romeo/).

6. Permission granted for books and journals is granted for the lifetime of the first edition and does not apply to second and subsequent editions (except where the first edition permission was granted free of charge or for signatories to the STM Permissions Guidelines http://www.stm-assoc.org/copyright-legalaffairs/permissions/permissions-guidelines/), and does not apply for editions in other 
languages unless additional translation rights have been granted separately in the licence.

7. Rights for additional components such as custom editions and derivatives require additional permission and may be subject to an additional fee. Please apply to Journalpermissions@springernature.com/bookpermissions@springernature.com for these rights.

8. The Licensor's permission must be acknowledged next to the licensed material in print. In electronic form, this acknowledgement must be visible at the same time as the figures/tables/illustrations or abstract, and must be hyperlinked to the journal/book's homepage. Our required acknowledgement format is in the Appendix below.

9. Use of the material for incidental promotional use, minor editing privileges (this does not include cropping, adapting, omitting material or any other changes that affect the meaning, intention or moral rights of the author) and copies for the disabled are permitted under this licence.

10. Minor adaptations of single figures (changes of format, colour and style) do not require the Licensor's approval. However, the adaptation should be credited as shown in Appendix below.

\section{Appendix - Acknowledgements:}

\section{For Journal Content:}

Reprinted by permission from [the Licensor]: [Journal Publisher (e.g. Nature/Springer/Palgrave)] [JOURNAL NAME] [REFERENCE

CITATION(Article name, Author(s) Name), [COPYRIGHT] (year of publication)

\section{For Advance Online Publication papers:}

Reprinted by permission from [the Licensor]: [Journal Publisher (e.g. Nature/Springer/Palgrave)] [JOURNAL NAME] [REFERENCE

CITATION(Article name, Author(s) Name), [COPYRIGHT] (year of publication), advance online publication, day month year (doi: 10.1038/sj.[JOURNAL ACRONYM].)

\section{For Adaptations/Translations:}

Adapted/Translated by permission from [the Licensor]: [Journal Publisher (e.g. Nature/Springer/Palgrave)] [JOURNAL NAME] [REFERENCE CITATION(Article name, Author(s) Name), [COPYRIGHT] (year of publication)

Note: For any republication from the British Journal of Cancer, the following credit line style applies: 
Reprinted/adapted/translated by permission from [the Licensor]: on behalf of Cancer Research UK: : [Journal Publisher (e.g. Nature/Springer/Palgrave)] [JOURNAL NAME] [REFERENCE CITATION (Article name, Author(s) Name), [COPYRIGHT] (year of publication)

For Advance Online Publication papers:

Reprinted by permission from The [the Licensor]: on behalf of Cancer Research UK: [Journal Publisher (e.g. Nature/Springer/Palgrave)] [JOURNAL NAME] [REFERENCE CITATION (Article name, Author(s) Name), [COPYRIGHT] (year of publication), advance online publication, day month year (doi:

10.1038/sj.[JOURNAL ACRONYM])

\section{For Book content:}

Reprinted/adapted by permission from [the Licensor]: [Book Publisher (e.g. Palgrave Macmillan, Springer etc) [Book Title] by [Book author(s)]

[COPYRIGHT] (year of publication)

\section{Other Conditions:}

Version 1.1

Questions? customercare@copyright.com or +1-855-239-3415 (toll free in the US) or +1-978-646-2777. 